\title{
Low Power Wireless CMOS System-on-Chip X-Ray Dosimeter
}

\author{
by \\ Behzad Yadegari, BASc, MASc \\ A dissertation submitted to the Faculty of Graduate and Postdoctoral Affairs \\ in partial fulfillment of the requirements for the degree of
}

\section{Doctor of Philosophy \\ in}

Electrical and Computer Engineering
Ottawa-Carleton Institute for Electrical and Computer Engineering
Department of Electronics
Carleton University
Ottawa, Ontario
January, 2019

Copyright (C) 2019

Behzad Yadegari 


\section{Abstract}

Blood sterilization systems irradiate blood with Gamma or X-ray radiation to prevent transfusion-associated graft versus host diseases (TA-GvHD).

Present day irradiators use tags applied to the blood bags which change color when exposed to radiation of 25 to $50 \mathrm{~Gy}$. This method is neither accurate nor efficient and results in considerable blood wastage.

This thesis investigates alternative solutions that are low power, easy to handle, com-

pact and efficient. A floating-gate (FG) metal-oxide semiconductor field effect transistor (MOSFET) is studied for its application as a radiation sensor. FG MOSFETs have been employed for this application, but never using an RF-CMOS $(0.13 \mu \mathrm{m})$ process suitable for RF-ID wireless readout. This work studies device optimization for best possible performance in radiation dosimetry. Devices are fabricated with different layer configurations to see how these affect sensitivity, linearity and power consumption. The final sensor measurements demonstrate a sensitivity of $6.5 \mu \mathrm{A} \mathrm{Gy}^{-1}$ with a maximum power consumption of $37 \mu \mathrm{W}$.

This work also explores novel sub-threshold operation of FG MOSFET sensors for this application. This technique is shown to reduce the power consumption of such sensors, hence improving their figure of merit. 
Additionally, FG MOSFET devices are configured as an inverter, rather than a standalone transistor, to work as radiation sensors. This provides great reduction in the power requirements and is completely novel. These sensors are seen to consume channel current in the range of $\mathrm{nA}$ in comparison to $\mu \mathrm{A}$ consumed by the single MOSFET, while providing acceptable sensitivity values. Furthermore, the thesis explores photodiodes as X-ray power harvesting devices which could be provided with the sensor chip itself for a battery-less solution.

Finally, an e-fuse RF-CMOS based non-volatile (NVM) memory is realized for tag identification and control in a multi-sensor context. The memory has been tested under irradiated conditions to show its operation in the proposed environment. The results demonstrate the system-on-chip implementation of key components of an electronic blood irradiator system in a small form factor, with ultra-low power consumption. The proposed solution can improve dosimeter accuracy, allow automation of the process, and reduce blood wastage. 
In memory of my grandmother and aunt;

For my lovely wife and teacher, Ariane;

For my parents and brother; 


\section{Acknowledgments}

This journey would not have been possible without the unconditional love and support I received from my lovely wife, Ariane, and my parents.

I would like to express my gratitude and respect to my supervisors Dr. Langis Roy and Dr. Steve McGarry for their continuous support, valuable suggestions, and precious advice. Without their guidance, this work would not have been accomplished.

I would also like to express my sincere thanks to Dr. Farhan Abdul Ghaffar for his essential role in assisting me with making corrections and modifications to this thesis as well as being there for me when I needed support the most.

I would like to sincerely thank all the helpful and supportive staff at the Department of Electronics at Carleton University, especially Mr. Nagui Mikhail for all the technical support.

Many thanks to all my friends and colleagues who throughout this research work, have contributed to my academic and personal life.

I would also like to acknowledge the great opportunity presented to me by Natural Sciences and Engineering Research Council of Canada (NSERC) to carryout this grand research to this stage. Their financial support, in the form of Engage and CRD grants, has had a pivotal role in the accomplishment of this work. 
I would like to thank Best Medical Canada Ltd. and Best Theratronics for their great partnership in this research work, through which I have received their financial support and have greatly benefited from their expertise.

Many thanks to Mr. Krish Suthanthiran, Mr. John Thomas, Mr. Rashid AlNader, Mr. Carlos Rodas, Mr. Andrei Ciresianu, Ms. Amanda Bates, and Ms. Angela Sanders who have made and continue to greatly support my research. 


\section{Table of Contents}

$\begin{array}{ll}\text { Abstract } & \text { ii }\end{array}$

Acknowledgments $\quad$ v

Table of Contents vii

List of Tables $\quad$ x

List of Figures $\quad$ xi

Abbreviations and Symbols $\quad$ xvii

1 Introduction 1

1.1 Motivation . . . . . . . . . . . . . . . . . . 1

1.2 Thesis Objectives . . . . . . . . . . . . . . . . . . . 4

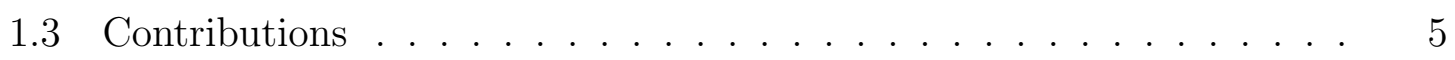

1.4 Thesis Outline . . . . . . . . . . . . . . . . . . . . . 8

2 Background and Literature Review $\quad 9$

2.1 The X-ray Dosimeter Project: A brief History . . . . . . . . . . 9

2.2 Overall Dosimeter System Description _. . . . . . . . . . . 10

2.3 Literature Review . . . . . . . . . . . . . . . . . . . . . 18 
2.3.1 Floating-Gate MOSFET Dosimeters . . . . . . . . . . . . . 18

2.3.2 Enhancement Techniques for Sensor Performance . . . . . . . 26

2.3.3 Non-Volatile Memory (NVM) Designs for RFID Applications . 31

2.4 Conclusion . . . . . . . . . . . . . . . . . . . . . 46

3 Floating-Gate MOSFET Dosimeter Design 48

3.1 Introduction . . . . . . . . . . . . . . . . . . . . 48

3.2 Principles of Operation: Radiation Sensing \& Programming . . . . . 49

3.3 Design Variants and Preliminary Results . . . . . . . . . . . . . . 50

3.3.1 Radiation Test Setups and Techniques . . . . . . . . . . . 51

3.3.2 Floating-Gate Extension with/without Silicide Layer . . . . . 57

3.3.3 Floating-Gate Extension with/without Metal Protection . . . 64

3.3.4 Floating-Gate MOSFET with/without Multiple Fingers . . . . 68

3.4 FG-MOS Charge/Discharge Cycles . . . . . . . . . . . . . . . 72

3.5 Sub-threshold MOSFET Dosimeter . . . . . . . . . . . . . 77

3.5.1 Principle of Operation . . . . . . . . . . . . . . 79

3.5.2 Sub-threshold MOSFET Design . . . . . . . . . . . . . 81

3.6 Chapter Conclusion . . . . . . . . . . . . . . . . . . . 85

4 Integration and Functionality Enhancements $\quad 87$

4.1 A Versatile FG-MOS Design . . . . . . . . . . . . . . . . . . . 88

4.2 Minimum Size Floating Gate Inverter . . . . . . . . . . . . . . . 95

4.3 X-ray Power Harvesting Using Diodes . . . . . . . . . . . . . . . . . . 104

4.3 .1 Photodiode theory ................. 106

4.4 Chapter Conclusion . . . . . . . . . . . . . . . . . . . . . . . . . . . . 119

5 Non-Volatile Memory Design $\quad 121$ 
5.1 Introduction . . . . . . . . . . . . . . . . . . . . . . . 121

5.2 Novel non-Volatile Memory Design and Implementation . . . . . . . . 123

5.2.1 Programming Circuit Design . . . . . . . . . . . . . . 128

5.2.2 Reading Circuit Design . . . . . . . . . . . . . . 131

5.2 .3 Layout . . . . . . . . . . . . . . . . . . 139

5.2 .4 Testing. . . . . . . . . . . . . . . . 141

6 Thesis Conclusions, Contributions, and Future Work 145

6.1 Thesis Conclusions and Contributions . . . . . . . . . . . . 145

6.2 Future Work . . . . . . . . . . . . . . . . . . . . . . . . . . . . . . 148

6.2.1 Future Work on FG-PMOS Devices . . . . . . . . . . . . . . . 148

6.2.2 Future Work on Integration and Functionality Enhancements . 149

6.2.3 One-Time Programmable Nonvolatile Memories . . . . . . . . 151

6.2.4 Publications . . . . . . . . . . . . . . . . . . 152

List of References 


\section{List of Tables}

2.1 Floating-gate dosimeter summary of the state of the art . . . . . . 25

3.1 Dimensions of MOSFETs fabricated for subthreshold irradiation experiments using the blood irradiator X-ray machine . . . . . . . . . 81

5.1 Input signals for write and read operations of the NVM . . . . . . . 126 


\section{List of Figures}

$1.1 \operatorname{RadTag}^{\circledR}$ sample label. . . . . . . . . . . . . . . . . . . . . . . . . 3

1.2 Raycell_MK2 ${ }^{\circledR}$ blood irradiation machine. . . . . . . . . . . . 4

2.1 Proposed system-level overview of the X-ray dosimeter project . . . . 12

2.2 System-level diagram of the X-ray dosimeter tag. . . . . . . . . . . 14

2.3 Cross section of FG MOSFET device . . . . . . . . . . . . . 21

2.4 Floating-gate dosimeter readout circuitry . . . . . . . . . . . 22

2.5 Cross section of the Semi-Floating-Gate MOSFET . . . . . . . . . . 24

2.6 Voltage Transfer Characteristics (VTC) of inverter design . . . . . . 28

$2.7 \quad$ FG Inverter design realized in $180 \mathrm{~nm}$ CMOS technology $\ldots \ldots$. . . . 29

2.8 Voltage response of FG-CMOS inverter design to gamma radiation from CLINAC; $V_{t}$ is the switching voltage of the inverter $\ldots \ldots 29$

2.9 System Concept proposed for self-powered X-ray dosimeter . . . . . . 31

2.10 Antifuse memory unit architecture . . . . . . . . . . . 35

2.11 A complete breakdown and a quasibreakdown of the gate oxide . . . 36

2.12 DPROM matrix . . . . . . . . . . . . . . . . . 37

2.13 Capacitor and access transistor bitcell structure . . . . . . . 38

2.14 Cross section of the drift antifuse cell. . . . . . . . . . . . . . 39

2.15 Selected and non-selected bitcells during programming using a drift

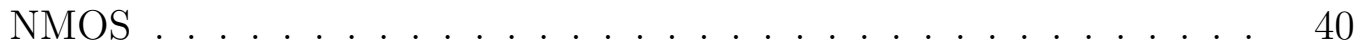


2.16 Access transistor cascoded to lower the drain voltage stress . . . . . . 41

2.17 Illustrating programming window . . . . . . . . . . . . . . . 41

2.18 A blown (right) and an unblown (left) eFuse structure . . . . . . . . . 42

2.19 Single-ended sensing . . . . . . . . . . . . . . . . . 43

2.20 Differential True/Complement eFuse sensing circuit . . . . . . . . . . 44

2.21 Shared reference sensing circuit . . . . . . . . . . . . . 46

2.22 A differential sense amplifier with programmable reference . . . . . . 47

2.23 A differential sense amplifier with programmable reference . . . . . . 47

3.1 Simplified operation of an FG-MOS sensor . . . . . . . . . . . . 50

3.2 X-ray test setup . . . . . . . . . . . . . . . . . . 52

3.3 FG-MOS under radiation in the Faxitron X-ray machine . . . . . . 52

3.4 Blood irradiation machine test setup . . . . . . . . . . . . . 58

3.5 Blood irradiation machine test setup zoom . . . . . . . . . . . . 58

3.6 Silicided (left) and non-silicided (right) poly-silicon gate extensions . 59

3.7 Depiction of silicided (left) and non-silicided (right) poly-silicon gate extensions ......................... 60

3.8 Schematic of the pair of FG-MOS devices . . . . . . . . . . . . . . 60

3.9 Radiation exposure of silicided and silicide-blocked devices to $110 \mathrm{kVp} \quad 61$

3.10 Radiation exposure of silicided vs. silicide-blocked device on a single chip using the Raycell Blood Irradiation Unit . . . . . . . . . . . 63

3.11 Grounded top metal layer covering the FG extension (left) and the exposed FG extension (right) . . . . . . . . . . . . .

3.12 The depiction of the grounded top metal layer covering the FG extension (left) and the exposed FG extension (right) . . . . . . . . . . 66

3.13 Metal shielded and no metal shield covered FG sensor exposed to $110 \mathrm{kVp}$ X-ray . . . . . . . . . . . . . . . . 
3.14 Metal shielded and no metal shield covered FG sensor exposed to X-ray from blood irradiator machine . . . . . . . . . . . . . 68

3.15 Capacitor model for the FG-MOS device . . . . . . . . . . . . . . 69

3.16 Single transistor (left) vs. multi-finger transistor (right) FG-MOS . . 70

3.17 Single-finger and multiple-fingers structures exposed to $110 \mathrm{kVp}$ X-ray 71

3.18 Single-finger and multiple-fingers structures exposed to X-ray from the blood irradiator machine . . . . . . . . . . . . . . . . . . 72

3.19 Discharging process of an FG MOSFET by applying different pulse heights and numbers. . . . . . . . . . . . . . . . . 74

3.20 Manual precharging . . . . . . . . . . . . . . . 76

3.21 Precharging process of an FG MOSFET by applying different number of pulses. . . . . . . . . . . . . . . . . . . . . 77

3.22 Precharging repeatability . . . . . . . . . . . . . . . . . . 78

3.23 NMOS transistors fabricated . . . . . . . . . . . . . . . . 82

3.24 PMOS transistors fabricated . . . . . . . . . . . . . . . . 82

3.25 Sample Layout of fabricated PMOS devices . . . . . . . . . . . . . . . 82

3.26 Voltage-controlled ring oscillator schematic . . . . . . . . . . 83

3.27 Device\#6 channel current change as per radiation . . . . . . . . . . . 84

4.1 Versatile floating-gate cell . . . . . . . . . . . . . . . 89

4.2 Small gate-connected NMOS $I_{D S}$ vs $V_{D S}$ curves (measured) . . . . . 91

4.3 Small gate-connected NMOS $I_{D S}$ vs $V_{G S}$ curves (measured) . . . . . . 91

4.4 Small gate-connected PMOS $I_{D S}$ vs $V_{D S} \ldots \ldots$. . . . . . . . . . . . 92

4.5 A sample of minimum size FG-NMOS device and its gate-connected reference biased at $1.0 \mathrm{~V}$ exposed to $110 \mathrm{kVp}$ X-ray . . . . . . . . . . 93

4.6 A sample of minimum size FG-NMOS device and its gate-connected reference biased at $0.1 \mathrm{~V}$ exposed to $110 \mathrm{kVp}$ X-ray. . . . . . . . . 
4.7 A sample of minimum size FG-NMOS device and its gate-connected reference biased at $1.0 \mathrm{~V}$ exposed to blood irradiator machine X-ray. . 94

4.8 An inverter transfer characteristics in different corners and temperatures $($ simulation $) \ldots \ldots . \ldots . \ldots . \ldots 97$

4.9 Radiation behavior of an FG-inverter biased at $0.5 \mathrm{~V}$ supply in the blood irradiator machine . . . . . . . . . . . . . . . . . 98

4.10 Radiation Behavior of an FG-inverter powered by $0.7 \mathrm{~V}$ and $1.0 \mathrm{~V}$ supplies in the blood irradiator machine . . . . . . . . . . . . . 100

4.11 Identical minimum size $\mathrm{N}$ and PMOS devices externally connected to created the inverter . . . . . . . . . . . . . . . . 102

4.12 Gate connected inverters transfer characteristics and current consumptions at different $V_{D D} \mathrm{~s} \ldots \ldots \ldots$

4.13 Discrete p-i-n diode dimensions . . . . . . . . . . . . . . . . . . . . 105

4.14 Discrete diode spectral response across the spectrum . . . . . . . 106

4.15 Photovoltaic operation of a diode . . . . . . . . . . . . 108

$4.16 \mathrm{P} \max \ldots \ldots \ldots \ldots \ldots \ldots$

4.17 Silicon absorption coefficient at 300k . . . . . . . . . . . . . . . 110

4.18 Silicon absorption depth at 300k . . . . . . . . . . . . . . . 111

4.19 Silicon mass energy abosrption coefficient . . . . . . . . . . . . . . . . 112

4.20 Scintillation of X-rays and consequent photo detection and conversion to electrical signal . . . . . . . . . . . . . . . . . . . . 113

4.21 Packaged discrete diodes with or without scintillating material . . . . 115

4.22 Discrete diode packages $1,2,3$, and 6 under $110 \mathrm{kVp}$ X-ray source irra-

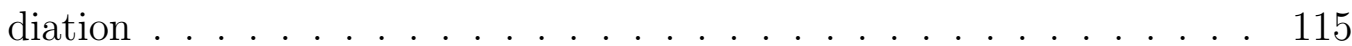

4.23 Generated power under 110kVp curve of D6 package . . . . . . . . 116 
4.24 Diode packages D1, D2, D3, and D6 irradiation using the blood irradiator machine . . . . . . . . . . . . . . . . . 117

4.25 I-V characterisitics of series and parallel combinations of 3 diodes in

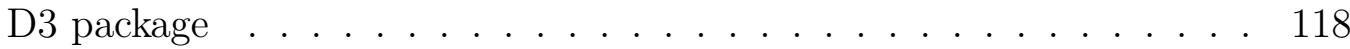

4.26 Power generated in a series and parallel combination of D3 diodes under blood irradiator radiation . . . . . . . . . . . . . . . 119

5.1 A microscopic image of the overall ID chip. . . . . . . . . . . . . . 125

5.2 Overall memory chip layout . . . . . . . . . . . . . . . . 126

5.3 Reading in the 8 bit data pattern to the registers to be written to the

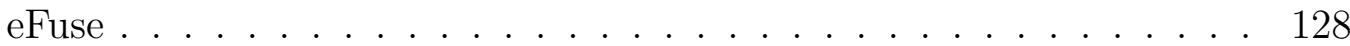

5.4 eFuse links and corresponding programming circuitry . . . . . . . . . 129

5.5 eFuse programming system block . . . . . . . . . . . . . . . . 129

5.6 "Fuse Cell" circuit block which controls sensing and programming . . 130

5.7 Fuse writing operation signal samples . . . . . . . . . . . . . . . . . 132

5.8 Supply independent current source for reading operation . . . . . . . 133

5.9 Clocked strong arm latch comparator for sensing . . . . . . . . . . . . 134

5.10 Readout block: The strong arm latch clocked comparator layout . . . 134

5.11 Read operation signal flow . . . . . . . . . . . . . . . . . 136

5.12 Supply independent current source . . . . . . . . . . . . . . . 137

5.13 DC current flow through the current source block for reading of a low resistance (connected) fuse . . . . . . . . . . . . . . . . . 138

5.14 DC current flow through the current source block for reading of a blown (high resistance) fuse . . . . . . . . . . . . . . . . . 139

5.15 DC values of VRef, VFuse, and Vbias in the case of a low resistance fuse as per $V_{D D}$ sweep . . . . . . . . . . . . . . . . . . 140 
5.16 DC values of VRef, VFuse, and Vbias in the case of a blown (high resistance) fuse as per $V_{\text {DD }}$ sweep . . . . . . . . . . . . . . . . . . . . 141

5.17 Zoomed-in layout of the ID block . . . . . . . . . . . . . . . 142

5.18 A sample read operation of the ID block. . . . . . . . . . . 143

5.19 Memory test setup . . . . . . . . . . . . . . . . . . 143

5.20 Clock signal supply reduction and operation of the chip in read mode 144

6.1 Comparator based 4-bit resolution FG-dosimeter with zero static power

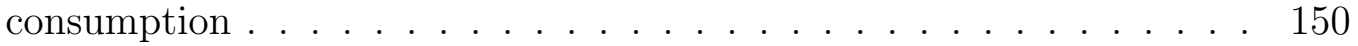




\section{Abbreviations and Symbols}

\begin{tabular}{|c|c|}
\hline ASK & Amplitude-Shift Keying \\
\hline $\mathrm{BMC}$ & Best Medical Canada Ltd. \\
\hline $\mathrm{C}$ & Capacitance \\
\hline $\mathrm{C}_{\mathrm{fb}}$ & Capacitance from Floating Gate to Bulk \\
\hline $\mathrm{C}_{\mathrm{fd}}$ & Capacitance from Floating Gate to Drain \\
\hline $\mathrm{C}_{\mathrm{fs}}$ & Capacitance from Floating Gate to Source \\
\hline CMOS & Complementary Metal-Oxide Semiconductor \\
\hline${ }^{60} \mathrm{Co}$ & Cobalt-60 \\
\hline $\mathrm{C}_{\mathrm{OX}}$ & Capacitance of Oxide \\
\hline $\mathrm{C}_{\text {sum }}$ & Sum of Capacitances \\
\hline $\mathrm{CW}$ & Continuous Wave \\
\hline $\mathrm{D}$ & Radiation Dose \\
\hline $\mathrm{DC}$ & Direct Current \\
\hline DRAM & Dynamic Random-Access Memory \\
\hline eFuse & Electronic Fuse \\
\hline $\mathrm{e}-\mathrm{h}$ & Electron-Hole pair \\
\hline$\epsilon_{\mathrm{OX}}$ & Permittivity of Oxide \\
\hline FG & Floating-Gate \\
\hline FG-MOSFET & Floating-gate Metal-Oxide Semiconductor Field Effect Transistor \\
\hline FSK & Frequency-Shift Keying \\
\hline$\gamma$-ray & Gamma radiation (High Energy Photons) \\
\hline Gy & Gray \\
\hline $\mathrm{IC}$ & Integrated Circuit \\
\hline $\mathrm{I}_{\mathrm{D}}$ & Drain Current \\
\hline $\mathrm{k}$ & Boltzman Constant \\
\hline MOSFET & Metal-Oxide Semiconductor Field Effect Transistor \\
\hline$\mu$ & Charge mobility or micro (unit) \\
\hline NMOS & n-Type Metal-Oxide Semiconductor \\
\hline NVM & Non-Volatile Memory \\
\hline OTP & One-Time Programmable memory \\
\hline
\end{tabular}




$\begin{array}{ll}\text { PIN } & \text { p-Type - intrinsic - n-Type diode } \\ \text { PMOS } & \text { p-Type Metal-Oxide Semiconductor } \\ \text { q } & \text { Charge of an electron } \\ \text { RF } & \text { Radio Frequency } \\ \text { RFID } & \text { Radio Frequency Identification } \\ \text { SFG } & \text { Semi-Floating Gate } \\ \text { Si } & \text { Silicon } \\ \text { SiP } & \text { System-in-Package } \\ \text { SoC } & \text { System-on-Chip } \\ \text { SPE } & \text { Signal Processing Electronics } \\ \text { SRAM } & \text { Static Random-Access Memory } \\ \text { STI } & \text { Shallow Trench Isolation } \\ \text { T } & \text { Temperature in Kelvin } \\ \text { TA-GvHD } & \text { Transfusion-Associated Graft-versus-Host Disease } \\ \text { TID } & \text { Total Ionizing Dose } \\ \text { UHF } & \text { Ultra High Frequency } \\ \text { V } & \text { Transistor Threshold Voltage }\end{array}$




\section{Chapter 1}

\section{Introduction}

\subsection{Motivation}

The demand for medical sensors with diverse functionality has been on the rise in the last few decades. In a recent article published in 2016 it was estimated that the market will continue to experience a dramatic increase in the utilization of these sensors for the foreseeable future [1]. This kind of a rise is in step with the continuous innovations in sensor design pertaining to the medical industry. Furthermore, the novelty that these sensors bring into health-care operations is invaluable. These sensors find their use in many applications, including X-ray imaging and radiation dosimetry [2]. Radiation dosimetry pertains to the class of sensors used for the accurate measurement of X-ray and gamma radiations. Such emissions are classified as ionizing radiation due to their disruptive impact on the subject [3]. As a result, these sensors are deemed to be highly critical and are applied to sensitive procedures such as cancer treatment and blood irradiation for sterilization. The economical and medical benefits of these incoming technologies have motivated the practitioners to use them more frequently, especially the radiation dosimeters [4], hence opening a new 
door for researchers to come up with better designs which meet the requirements.

\section{Blood Sterilization}

The advent of blood transfusion has significantly impacted the medical industry by greatly increasing the number of lives that can be saved. However, the process in itself brings a list of challenges which need to be considered. Among them is the existence of bloodborne conditions that can eventually be fatal. Hence, there are stringent steps in screening of the relevant donors and sterilization of the blood itself. It has been observed that in the absence of proper sterilization serious diseases such as hepatitis B \& C, syphilis and several Human Immunodeficiency Virus (HIV) can be transmitted due to blood transfusion [5]. To cater to this a number of sterilization methods are in place at present. The most popular among these is irradiation due to its effectiveness against the transfusion-associated graft-versus-host disease (TAGvHD) [6]. The seriousness of TA-GvHD can be understood from the fact that the overwhelming majority of the patients suffering from it will eventually die. This group of patients include transplant patients, bone marrow recipients, cancer patients, neonates, and persons with congenital immune deficiency syndromes. This highlights the significance of blood sterilization. In general, X-ray or gamma radiations are used to sterilize the donated blood. When exposed to the right amount of radiation, the stored blood can be made safe for transfusion purposes [6]. $\operatorname{RadTag}^{\circledR}$ is one of the most common products that is currently employed for blood product irradiation and is shown in Figure 1.1 [7].

The $\operatorname{RadTag}{ }^{\circledR}$ system can help in identifying the level of radiation the blood has been exposed to by using radiation-sensitive tags. These tags are placed on the top of each blood bag that is stored in the system. Between 15 Gy and 50 Gy of dose the radiation 


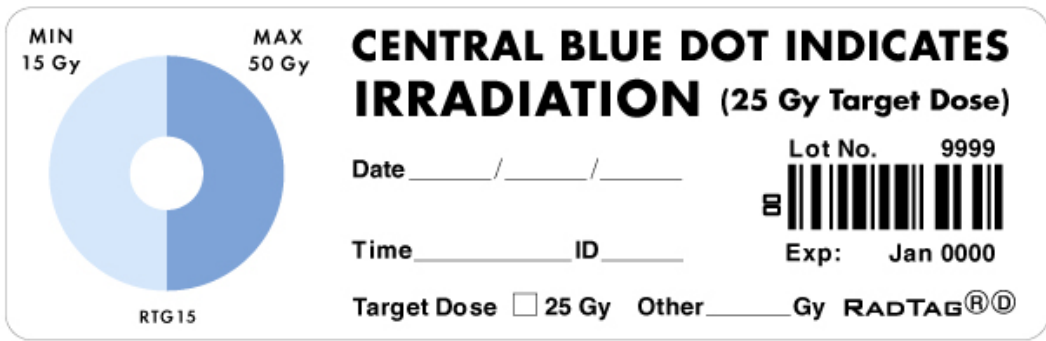

Figure 1.1: $\operatorname{RadTag}{ }^{\circledR}$ sample label. The white circle in the middle gradually turns blue with cumulative radiation [7].

exposure can be estimated using these tags. However, there are two problems with this method: 1) the process is binary as it cannot estimate the radiation levels in between the two extremes and 2) the method is significantly prone to human errors as it relies on visual verification of color change. These deficiencies can be done-away with by using electronic systems that can accurately estimate the radiation levels without any human errors.

Best Theratronics, located in Ottawa, Ontario, manufactures these blood irradiators for both research and commercial applications. A consortium of companies under the umbrella of Team Best includes both Best Teratronics and Best Medical. This research is carried out in collaboration with Best Medical. Best Theratronics is one of the global leaders in the blood irradiator industry. Gammacell and Raycell brands are the two major products of this industry which are used for blood sterilization purposes $[8,9]$. In Figure 1.2, a diagrammatic representation of Raycell Mk2 is shown. The product comprises X-ray tubes which act as the source of the radiation. Blood bags are placed into either a $2.0 \mathrm{~L}$ or $3.5 \mathrm{~L}$ canister inside the machine. Either 300 $\mathrm{mL}$ or $600 \mathrm{~mL}$ of blood is contained in each bag. At a time, the machine is capable of exposing 2-8 blood bags to X-ray radiation. The flexibility in the number of bags is due to the size of both the bags themselves and the canister. The bags are exposed to X-ray radiations for a period of 2.8 or 5.7 minutes in order to ensure that 25 
Gy of radiation level has been achieved. Dose uniformity varies between 1.8:1 and 1.5:1 $[8,9]$. This is a drawback of this system as stated previously. If an electronic sensor is used as opposed to these tags then the dose strength can be monitored with better precision hence improving the overall efficiency in terms of cost, blood wastage and certainty of the radiation levels the product has been exposed to.

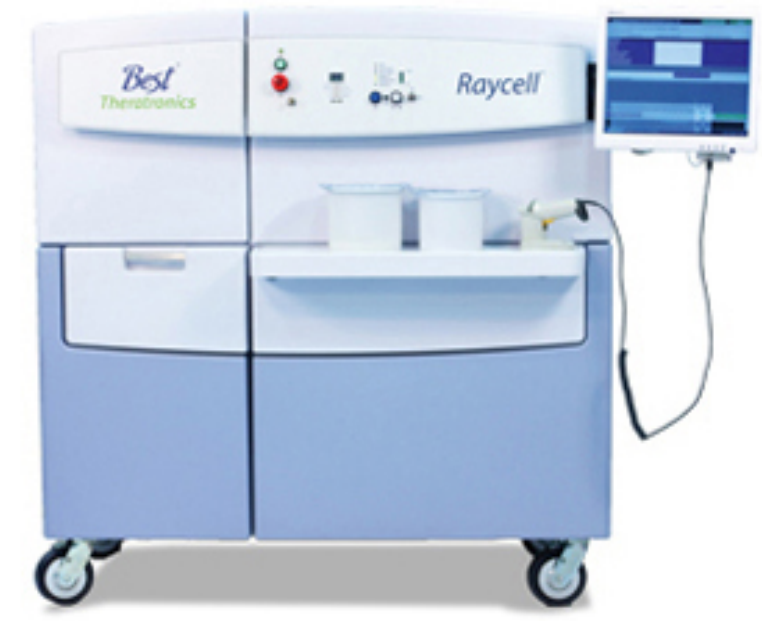

Figure 1.2: Raycell_MK2 $^{\circledR}$ blood irradiation machine. [9]

\subsection{Thesis Objectives}

In the context of the goals described in the last section, this thesis focuses on the design of wireless X-ray dosimetry based on an RFID-compatible System-on-Chip (SoC) technology. Broadly speaking, the five main research objectives of this work are:

Objective 1. Investigation, design, and full characterization of a floating-gate radiation dosimeter in the $0.13 \mu \mathrm{m}$ RF-CMOS technology to be tested using the abovementioned Raycell ${ }^{\circledR}$ blood irradiation machine. The floating-gate (FG) MOSFET sensor must be sensitive to photon energies emitted by the Raycell X-ray 
blood irradiation machine and must also be able to maintain the charge preand post-irradiation.

Objective 2. Design and full characterization of an alternative non-floating-gate, radiation dosimeter in the same technology for the sub-threshold region of operation, in order to enable a lower power implementation of the dosimeter function.

Objective 3. Design, implementation, and characterization of a second alternative to reduce power consumption: that of a low power floating-gate inverter as a digital radiation sensor.

Objective 4. Investigation of X-ray energy harvesting using diodes coated with scintillating materials, as a possible enhancement to the power efficiency of the proposed solution. This will set the direction for future battery-less designs.

Objective 5. Design and implementation of a non-charge-based non-volatile 8-bit memory block in order to identify each individual dosimeter chip. It is essential that this block operates under radiation without being adversely affected and with minimum power consumption.

In addition, this thesis aims to set the stage for research into a number of functionality enhancements and for complete SoC integration, by exploring related future concepts.

\subsection{Contributions}

Consistent with the objectives laid out for this research, the following contributions are made in this thesis:

1. A complete study on the application of FG MOSFET in an RFID/RF-CMOS technology as an irradiation sensor for a variety of design variables has been 
undertaken in this work. It is shown how the presence of a silicide passivation layer has an effect on the device performance (sensitivity) whereas a grounded metal layer covering the floating-gate extension used as a shield against electromagnetic noise does not significantly affect its behavior. In addition, it has been deduced that the use of multiple gate fingers as opposed to a single gate finger does not have any impact on the sensitivity of the device. The final results show a maximum sensitivity of $6.5 \mu \mathrm{A} \mathrm{Gy}^{-1}$ for the first $100 \mathrm{~s}$ equivalent of $10.4 \mathrm{~Gy}$ of the irradiation using the blood irradiator machine when the FG-PMOS is charged to $300 \mu \mathrm{A}$ for a constant DC bias of $-0.1 \mathrm{~V}$.

2. In addition to conventional MOS operation above the threshold voltage, it is also shown that the sub-threshold operation of the device can be exploited for lower power operation. This technique remains to be optimized but it does provide a pathway for investigation into low power designs.

3. For the first time an FG-CMOS inverter design is investigated for its realization in a commercial RFID integrated chip technology in the radiation dosimetry. The design provides an extremely low power solution for the desired sensor application. The topology thus presented shows a variable sensitivity with a resolution of 1 bit with a maximum current draw of $600 \mathrm{nA}$ at $\mathrm{V}_{\mathrm{DD}}=1.0 \mathrm{~V}$ for the duration of the output voltage transition. Compared to the conventional FG-PMOS dosimeter which consumes an average of $18 \mu \mathrm{W}$ during a typical irradiation of $5 \mathrm{~min}$, this design consumes $600 \mathrm{nW}$ of power during the transition period of approximately $50 \mathrm{~s}$.

4. To further enhance the capabilities of the proposed sensing technology an energy harvesting device is proposed to introduce the concept of an autonomous battery-less sensor. Off-the-shelf discrete photodiodes are employed to work as 
an energy harvesting circuit. When exposed to X-rays the diodes absorb their energy to generate the required charge in the channel. This generated energy by the photodiodes can potentially be used to supply the power to the MOSFET sensors proposed herein. The same design concept can be implemented using on-chip diodes to have seamless integration with the CMOS sensors.

5. A low power 8-bit eFuse memory block with read and write capabilities is designed and fabricated, and its operation has been verified under irradiation exposure duration of 20 minutes equivalent of $125.2 \mathrm{~Gy}$. The unique design enables the cell to operate (in read mode) with a bias range of $0.53 \mathrm{~V}$ to the nominal $1.2 \mathrm{~V}$. Minimum power consumption of $63 \mu \mathrm{W}$ is drawn during the read operation. Another unique feature is the technique used to isolate the high voltage, high current eFuse burning circuitry from the sensing circuitry. This is essential since standard transistors are not fabricated to tolerate voltages in the range of $3.3 \mathrm{~V}$ and currents close to $15 \mathrm{~mA}$ due to possible damage to the read circuit block during the burning operation.

6. Based on the above contributions, the following journal publications are foreseen in the future:

(a) B. Yadegari, et. al., "A novel X-ray CMOS Based Ultra-Low Power Wireless Dosimeter System for Blood Sterilization Applications", IEEE Journal on Circuit and Systems, 2019 (under preparation)

(b) B. Yadegari, et. al., "Low power Floating-Gate PMOS X-Ray Radiation Sensor", IEEE Transactions on Electron Devices, 2019 (under preparation)

(c) B. Yadegari, et. al., "Ultra-Low power Chip ID Block Developed for X-Ray Radiation Sensor for Blood Sterilization", IEEE Transactions on Electron 
Devices, 2019 (under preparation)

(d) B. Yadegari, et. al., "A Novel X-Ray Radiation Power Harvester Block Utilizing On-Chip PN Junctions", IEEE Transactions on Nuclear Science, 2019 (under preparation)

\subsection{Thesis Outline}

Chapter 2 provides thesis background and a detailed literature review relevant to the objectives and contributions of the thesis. This chapter will lay down the foundation and define the scope of the work. Chapter 3 presents work on the first and the second thesis objectives, floating-gate MOSFET dosimeter under conventional operation and its evaluation as a sensor in sub-threshold region of bias. The aim of Chapter 4 is to provide techniques where the FG MOSFET technology can be improved in terms of the power efficiency. For this purpose a new concept of using CMOS inverters as sensors will be studied while explaining techniques to harvest energy using the radiation exposure. Chapter 5 overviews the work on the final thesis objective, the NVM (Non-volatile memory) development and its complete characterization for the desired application. Finally, Chapter 6 presents the expected outcomes of the thesis while directing towards the future work that can be done in the area of radiation dosimetry. 


\section{Chapter 2}

\section{Background and Literature Review}

\subsection{The X-ray Dosimeter Project: A brief History}

Wireless dosimetry research is not new to Carleton University. In the past few decades the university has contributed significantly to the topic and has demonstrated considerable progress in the development of this area for the medical industry. In the mid-1980s research was initiated at Carleton with the help from Thomson and Nielson Electronics Ltd, providing a the direct practical application of the work. The fundamental research in this direction was carried out with the development of novel floating-gate (FG) metal-oxide semiconductor field effect transistor (MOSFET) in radiation dosimetry. The successful outcome of the initial endeavors of the professors and the graduate students resulted in the continuation of this work up to today. In the intervening years, Prof. N. Garry Tarr took the lead on this topic. As a result, the first milestone publication came out from the group in 1996. The paper demonstrated a novel FG-MOSFET, which was used for measuring gamma radiation [10]. The main focus of this work was to improve the sensitivity of the device when exposed to gamma rays. This was achieved by extending the polysilicon gate over the field 
oxide region thus increasing the area for the charge capture. The measured results thus exhibit the viability of the technique by showing much improved sensitivity of the device. The devices used in this work were realized in $5 \mu \mathrm{m}$ complementary metaloxide semiconductor (CMOS) technology. Naturally the channel size used in [10] is supposed to reduce in the later years as the CMOS technology advances. Therefore, in 1998 a similar device was realized in 1.5 $\mu \mathrm{m}$ CMOS process [11]. While the device further provided the proof-of-concept, it also showed greatly enhanced sensitivity i.e., four times of the one reported in [10]. These initial feats triggered further enhancement in the dosimeter design with the integration of sensing and reference transistors while adding functionalities such as temperature compensation [12]. In the early $21^{\text {st }}$ century, PhD students A. Shamim and M. Arsalan continued this work under the supervision of Profs. Langis Roy, Maitham Shams and N. Garry Tarr. Their focus was to study the effect on the performance and efficiency of the dosimeter device while using different circuit architectures and employing diverse packaging options. In addition, they extended the device-level research to a system level design by showing a complete wireless sensor system for radiation dosimetry. During their tenure at Carleton University, the pair of researchers developed novel System-on-Chip (SoC) and System-in-Package ( $\mathrm{SiP})$ transmitters and receivers specifically designed for MOSFET dosimeters [13-16].

\subsection{Overall Dosimeter System Description}

Before moving to the actual designs that are to be investigated in this thesis, it is imperative that the current X-ray dosimeter system is explored in detail. This will help to gain a more fulsome perspective of the research. This section covers the system concept while explaining the environment in which the blood bags are to be 
kept. Following this, an overview of the tag with its details is presented that covers its various components and their functionalities. At the end, an initial discussion of the reader is also included.

As it has been discussed in the last section that the synopsis of this work is to design a wireless dosimeter tag that can electronically measure the radiation levels that the stored blood bags have been exposed to. These integrated RFID tags are proposed as a substitute to the barcode labels which are currently used by Best Medical Canada. The proposed dosimeter can provide an exact amount of ionizing radiations that are received during blood sterilization process. Best Theratronics Raycell Mk2 blood irradiator unit, shown in Figure 1.2, is where the tag is to be eventually used. Thus, all the design steps should cater for the limitations of this setup.

\section{Dosimeter System}

A diagrammatic representation of the dosimeter system is illustrated in Figure 2.1 [17]. The system comes with a canister, the volume of which determines the size and the number of the blood bags that can be used. A compact portable reader is placed inside the Raycell MK2 system with the help of which one can observe the system parameters. In order for the reader to build a communication link with the tags, a small gap is provided at the top right corner of the chamber. This opening ensures the reader to communicate with the tags for a distance of around one meter. Since the tags are placed altogether, a unique identifier code/value is hard-coded into the tag's circuit to distinguish it from the others. This allows the reader to communicate with one particular tag at a time by virtue of a UHF based ASK-modulated signal at $2.45 \mathrm{GHz}$. As the interrogating signal contains the ID code of one tag all the other tags are virtually unhinged by the incoming RF signal. The tags are programmed in a way that on receiving the ASK signal, they compare it to their ID values once the 
signal has been demodulated. If the stored ID matches with the received one, the tag backscatters the signal with the embedded information of sensed X-ray dose and the temperature levels. The reader establishes a backscatter link by broadcasting a 2.45 GHz CW carrier tone. The tag under communication modulates its data on the CW carrier using PSK modulation and reflect the signal back to the reader. In this way, the reader is able to pick the data of one particular tag as the other tags will become dormant as soon as a mismatch between their ID and received ID has been established.

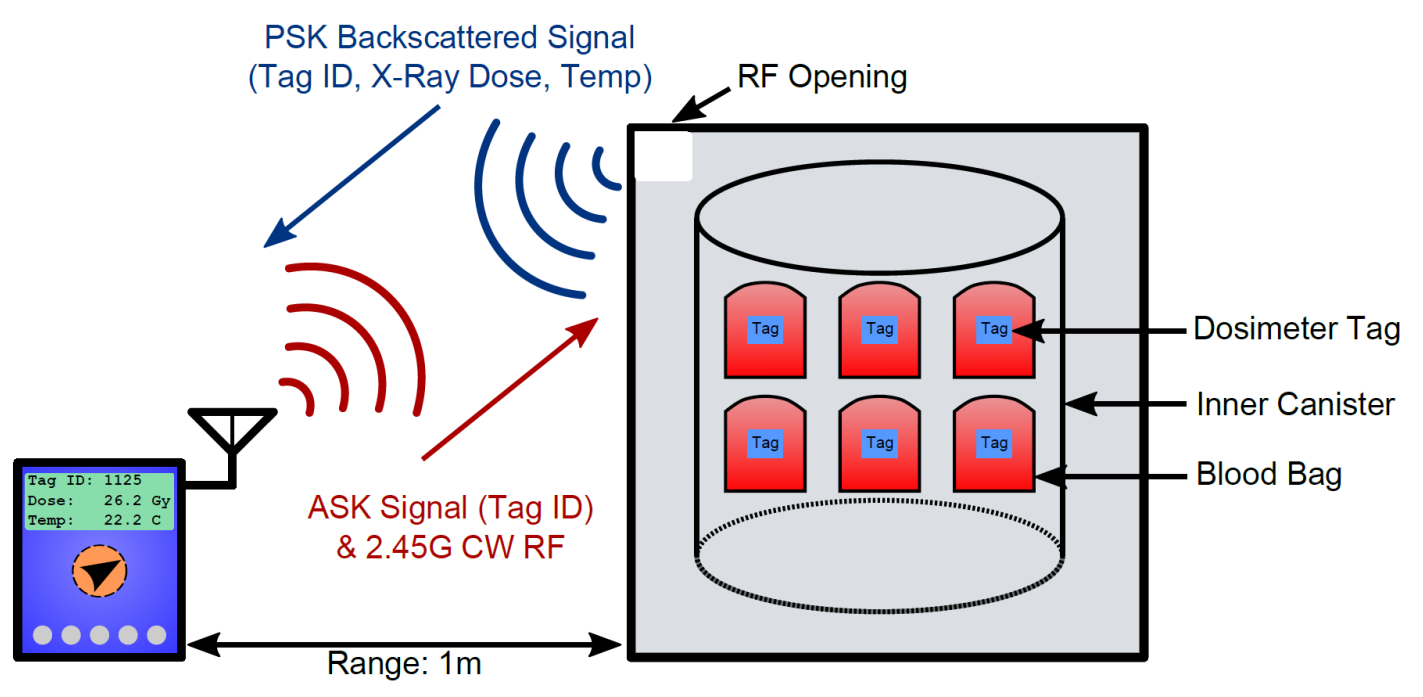

Dosimeter Reader

Raycell Mk2 Irradiation Chamber

Figure 2.1: Proposed system-level overview of the X-ray dosimeter project. Blood bags are affixed with RFID dosimeter tags and placed in the irradiation chamber. A reader, placed inside the Raycell $\mathrm{Mk}^{\circledR}{ }^{\circledR}$, measures the dosage of the RFID tags through a small opening in the side of the chamber. [17]

Although the remaining tags do not communicate with the reader, they still serve a special purpose. Instead of wasting this communication link, the system intelligently uses it for energy harvesting purposes. All the tags are designed to be equipped with an integrated energy harvesting circuitry. Thus, the radio frequency (RF) signal is collected by the tags to convert into a direct signal for harnessing energy. This energy 
is stored in a battery/storage device. The novel approach of using the RF signal as the energy source will greatly improve the efficiency of the system by intelligently applying it for dual purposes i.e. to communicate and store power.

\section{Dosimeter Operating Environment}

Any new technology that is designed for modern communication systems needs to be evaluated for its functionality in the designated operating environment. For instance, satellite systems require space grade qualification before they can be actually deployed. Similarly, the solutions to be used in the healthcare industry needs to be investigated in the variety of environments that include hospitals, storage refrigerators, and blood irradiation rooms. For this work, the dosimeter reader needs to be validated for two different modes of operation. In the first mode, the reader is installed inside the Raycell Mk2 while keeping it external to the irradiation chamber. This placement is depicted in Figure 2.1. In this configuration, the reader can monitor the X-ray dosage levels that has been applied during the sterilization process. For the second scenario, the reader would be external to the Raycell Mk2. A number of circumstances can be considered for this mode which include transportation, storage and other medical situations. Both these modes require the tag to operate well for a distance of up to one meter. This has to be maintained as one of the target requirements in the proposed solution.

\section{Dosimeter Tag Oveview}

An efficient, cost-effective and smart RFID based dosimeter tag is proposed herein. Using IBM's commercial 0.13 $\mu \mathrm{m}$ CMOS technology, the circuit level design of individual modules is to be carried out. By the virtue of energy harvesting, the design is made energy efficient. Backscatter modulation is used to convey the message from 
the tag to the interrogator. A complete system-level illustration of the tag is shown in Figure 2.2.

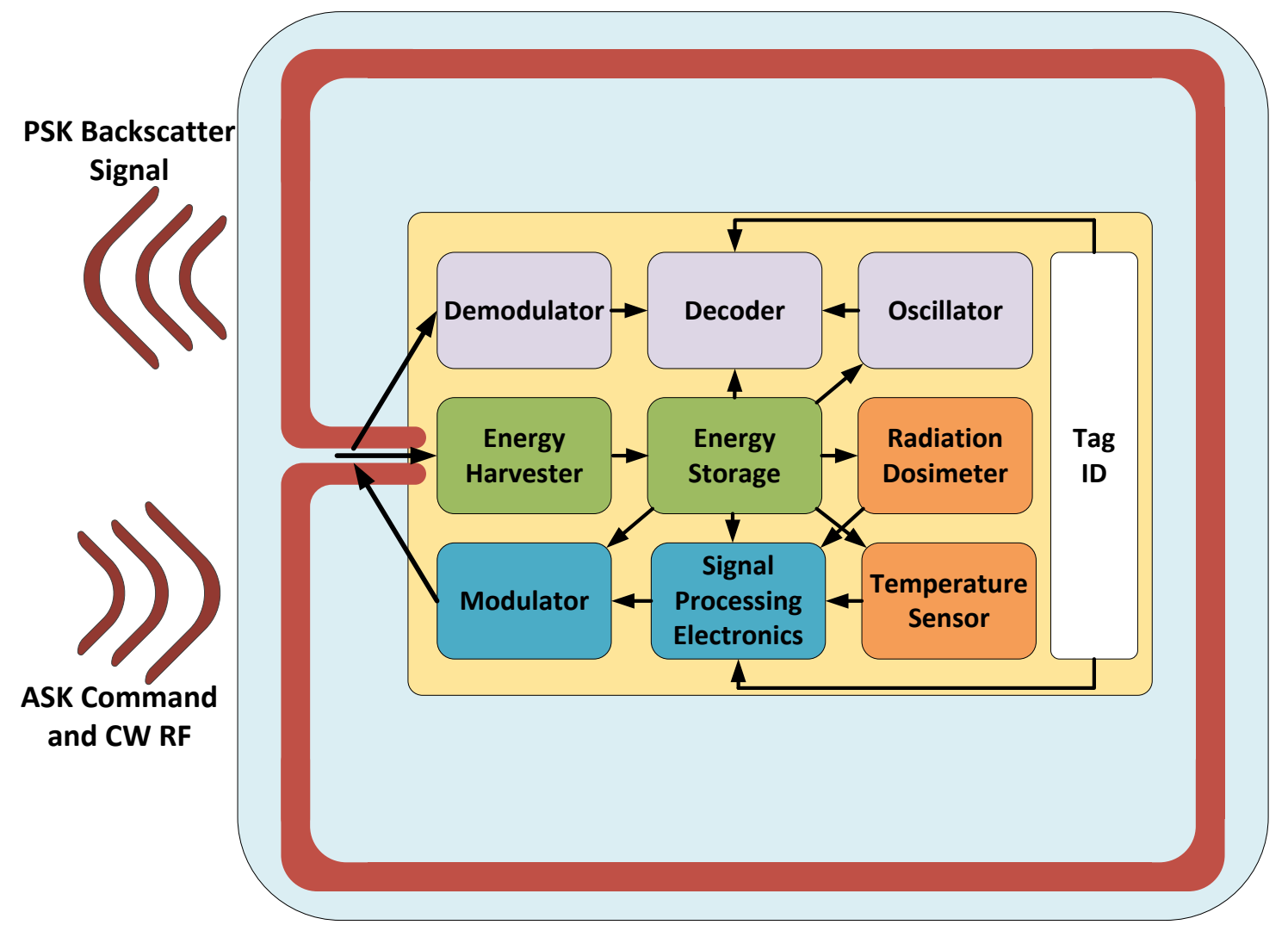

Figure 2.2: System-level diagram of the X-ray dosimeter tag. The sub-blocks are colour-coded to emphasize their shared functionality: purple corresponds to demodulation circuitry, green represents energy harvesting and storage components, orange corresponds to sensors, and blue signifies modulation circuitry. The antenna and tag ID are used by multiple sub-blocks. [17]

In order to compare the transmitted ID to the tag's ID, a demodulator, decoder and oscillator blocks are used and are shown in purple. An envelope detector is integrated in the system to work as a demodulator. The decoder may be implemented using combinational digital logic and a serial-to-parallel converter consisting of a series of flip-flops. For oscillator implementation, different techniques and circuit designs can be used. The purpose of the oscillator is to provide the clock signal which can be 
easily done by using a ring topology. An initial estimate of power budgeting shows that these sub-modules can operate efficiently with an overall consumption of $10 \mu \mathrm{W}$ which is quite reasonable [18].

The power source in the dosimeter tag is basically composed of two components, an RF energy harvester and an energy storage device. When an RF signal from the reader impinges on the antenna, a part of it is transmitted to the rectifier which is used as the energy harvester. The rectifier converts the alternating RF voltage to a direct current (DC) voltage. This DC voltage in turn charges the storage device (i.e. basically a capacitor). Once the device is sufficiently charged, it can provide the required energy to the sub-modules of the dosimeter tag for their dedicated operation.

An important functionality of these tags is to continuously monitor the temperature of the blood bags. It is a common practice for any medical facility to move these bags into and out of cold storage chambers. Thus, a significant change in temperature is experienced by these blood bags. A CMOS based temperature sensor is integrated in the system design that can provide the relevant information. The main challenge of this design is to keep it energy efficient. Literature shows that there are such designs that have been reported by CMOS designers [19]. Professor Cho led a team of researchers on the design of a ring oscillator-based temperature sensor in $0.13 \mu \mathrm{m}$ technology [20]. It is demonstrated that the sensor can fulfil the performance requirements with a total power consumption of $95 \mathrm{nW}$. Another temperature sensor designed in the same technology was proposed for RFID application in 2013 [21]. With the use of energy harvesting, the authors reported a power consumption of $8.5 \mu \mathrm{W}$. As a practical figure, this thesis presumes that the power consumption of the temperature sensor will be less than $10 \mu \mathrm{W}$. Temperature sensor design is not the major topic of focus and therefore a rigorous study is not really necessary at this 
stage. However, it will be taken up later as this is one of the key requirements of the dosimeter tag.

The presence of multi-functional modules in the complete system highlights the importance of the signal processing electronics (SPE) for the dosimeter tag. Modified Schmitt trigger topology is used for the SPE design. The use of SPE is to provide an interface between the (radiation and temperature) sensors and the modulator. The data retrieved from the sensors are translated into electrical voltage signals. These voltage levels are transformed into series of pulses. The frequency of the pulses is directly related to the magnitude of the voltage. With the frequencies in kilohertz, the pulses are digitized to be used as the data input for the modulator. The modulator uses the signals from the SPE as the information signal for the $2.45 \mathrm{GHz}$ carrier. The complete SPE design implementation is done at Carleton University by Mohamed Javad Sheikhzadeh Nadjar, a Masters student under Professor Langis Roy. The design shows a power dissipation of $100 \mathrm{nW}$ and is well within the estimated requirement.

For the dosimeter tag to communicate with the reader, PSK is used as the modulation scheme while the link is established by backscattering the RF signal generated by the reader itself. A simple transistor is employed in the system that provides the function of a high frequency switch. To control the switch, SPE circuitry generates the required train of pulses, which in turn varies the impedance loaded onto the tag antenna. This change in the impedance of the antenna is then used to understand the transmitted information. It is important here that any change in the load impedance does not necessarily reduce the efficiency of the system by dissipating some of the power. Therefore, the well-known reactive loading technique is employed in this work. Although the circuit designs are implemented in CMOS technology, it is not the best 
of the solutions to realize the antenna on-chip. The rationale for this strategy is the low gain and poor efficiency of such an antenna design. Thus, a better solution is to realize an off-chip antenna that can provide better radiation performance which helps in improving the range of communication [15]. A conventional dipole antenna is chosen to be integrated with the active circuitry. The antenna ensures a large effective area while providing differential operation which will allow its seamless integration with the CMOS system. The application requires the antenna to be flexible which is another challenge for this environment. To ensure this, a compact flexible substrate will be used for a $2.45 \mathrm{GHz}$ antenna realization. The substrate itself will provide the platform for the implementation of the complete dosimeter tag [22].

The next step is to make sure that the ID value of the tag is stored in a proper manner and can be recalled accordingly. There are several methods that can be used here but one has to keep in mind the targeted application requirements. For instance, the ID value should be completely impervious to the available power to avoid any errors. This means that a volatile memory solution (like static randomaccess memory SRAM and dynamic random-access memory DRAM) is not a viable option here. Another challenge in the memory design is the use of standard $0.13 \mu \mathrm{m}$ CMOS process. This process does not provide flash memory solutions. As a result of these issues, designers working in this area have come up with non-volatile memory (NVM) solutions $[23,24]$. There is one concern which should be considered with such memories: their poor resilience to ionizing radiation. This means that the memory can produce errors when exposed to TID values as low as 500 Gy [25]. Since the maximum power of X-ray radiation in Raycell Mk2 is $41 \mathrm{~Gy}$, this concern can be eliminated by limiting the re-use of the tags to one dozen irradiation procedures. A better solution will be to propose alternative memory solution for the storage of tag ID. Electronic fuse (eFuse) can provide the right solution in the IBM $0.13 \mu \mathrm{m}$ 
CMOS process for the stated purpose. An important feature of this memory circuit is its negligible power consumption which is one of the main objectives. A separate programming device is needed to apply a $3.3 \mathrm{~V}$ electric field across a portion of an array of eFuses, where each fuse represents a single storage bit [26]. The advantage of eFuse is its non-reversible writing process due to which the ID is permanently stored in it and will not be affected by the incoming ionizing radiation. Moreover, this solution can avoid any external tampering thus improving the overall security. With all these consideration taken into account, a one-time programmable (OTP) memory design is proposed for this work.

\subsection{Literature Review}

Before delving into the actual designs of this thesis, it is important to establish the state-of-the-art technologies that currently exists. In this section, a brief summary of the three enabling technologies pursued in the thesis is presented.

\subsubsection{Floating-Gate MOSFET Dosimeters}

The heart of this thesis is the advancement of FG (floating-gate) MOSFET dosimetry. MOSFETs, as will be presented shortly, have been researched extensively for radiation dosimetry applications. The sensitive part of the MOS structure is the oxide layer where high energy photons create electron-hole pairs and also permanent effects such as bulk and interface trap generation.

Earlier MOS structures had a relatively thick gate oxide layer $(\approx 100 \mathrm{~nm} \rightarrow 1 \mu \mathrm{m})$, depending on the technology node utilized. Unlike the focus of this thesis on the advancements on FG-MOS structures for radiation dosimetry, these older devices 
had a gate connection which applied a bias to the MOSFET during the irradiation session. Fundamentally, ionizing radiation creates energy traps as mentioned above within the volume of the oxide, which lead to a cumulative electric field generation. This radiation-caused electric field opposes or adds to the electric field applied at the gate depending on the polarity of the gate bias and that of the traps within the gate oxide.

Accumulation of the bulk and interface traps within the oxide has a direct relation with the cumulative radiation dose, such that a longer duration of radiation exposure causes more permanent traps within the oxide.

In order to take advantage of this characteristic of the MOSFET in dosimetry, voltages are applied across the drain and the source and to the gate terminals prior to irradiation and the channel current is measured. Maintaining the drain-source bias under radiation, it is desired to keep the channel current unchanged throughout the radiation session; therefore, the gate bias has to change to overcome the shift in the electric field caused by the trap generation. This change in the gate voltage can be interpreted as a shift in the threshold voltage of the MOS device as a result of radiation. When calibrated against an absolute dose measurement reference device, a shift in threshold voltage due to radiation is used to indirectly measure the absorbed radiation dose.

In modern MOS technologies, gate oxide thickness has shrunk to $\approx 5 \mathrm{~nm} \rightarrow 15 \mathrm{~nm}$ which practically desensitizes it to radiation due to a large reduction in interactions between the incident ionizing radiation photons and the oxide layer. Hence, the traditional techniques explained above would not be useful in radiation measurement.

The current approach to radiation measurement using MOS devices relies on the creation of electron-hole pairs within the oxide. But since the gate oxide is not thick 
enough for this phenomena, designers have utilized the large body of field oxide used to isolate gate polysilicon layer routing above the substrate. Any polysilicon layer outside the area of the MOS device needs to have isolation oxide layer underneath it. This oxide layer is generally thick $(\approx 350 \mathrm{~nm})$ in order to stop leakage from and onto the gate poly.

In order to use this characteristic of the MOS device, devices much like electrically erasable programmable read-only memory (EEPROM) are designed which operate based on charge retention on the gate. In this mode of operation of the MOS device, no gate bias is present and in fact, the MOS gate is electrically isolated from all surrounding connections. Through tunneling, excess charge (electrons or holes) is injected into the gate where they get trapped, and change the channel current when a voltage is applied across the drain-source terminals.

Radiation creates electron-hole pairs within the field oxide which recombine with the excess charge on the floating gate, hence, discharging the gate and causing a change in the channel current. A change in the current in such floating-gate devices is used to indirectly sense cumulative radiation absorption.

The state of the art of this field can be described by the following references:

1. One of the very first applications of FG MOSFET in radiation dosimetry was reported by Professor G. Tarr with his team in 1997 [10], as discussed in the beginning of this chapter. A more detailed publication was later published in IEEE Transactions on Nuclear Science in the subsequent year [11]. In this work, a p-channel MOSFET with an extended floating-gate arm was proposed, as shown in Figure 2.3. Exploiting this extended structure the device reported a large increment in the charge generation due to the exposure of external radiation. Such a technique can improve the sensitivity and the range of the 
radiation levels that can be eventually measured from the device. In addition, a matched pair of these FG MOSFET devices were employed thus removing any measurement errors that may incur due to temperature variations. The devices were realized using 1.5 $\mu \mathrm{m}$ CMOS process and demonstrated a measured sensitivity of $70 \mathrm{mV} \mathrm{Gy}^{-1}$.

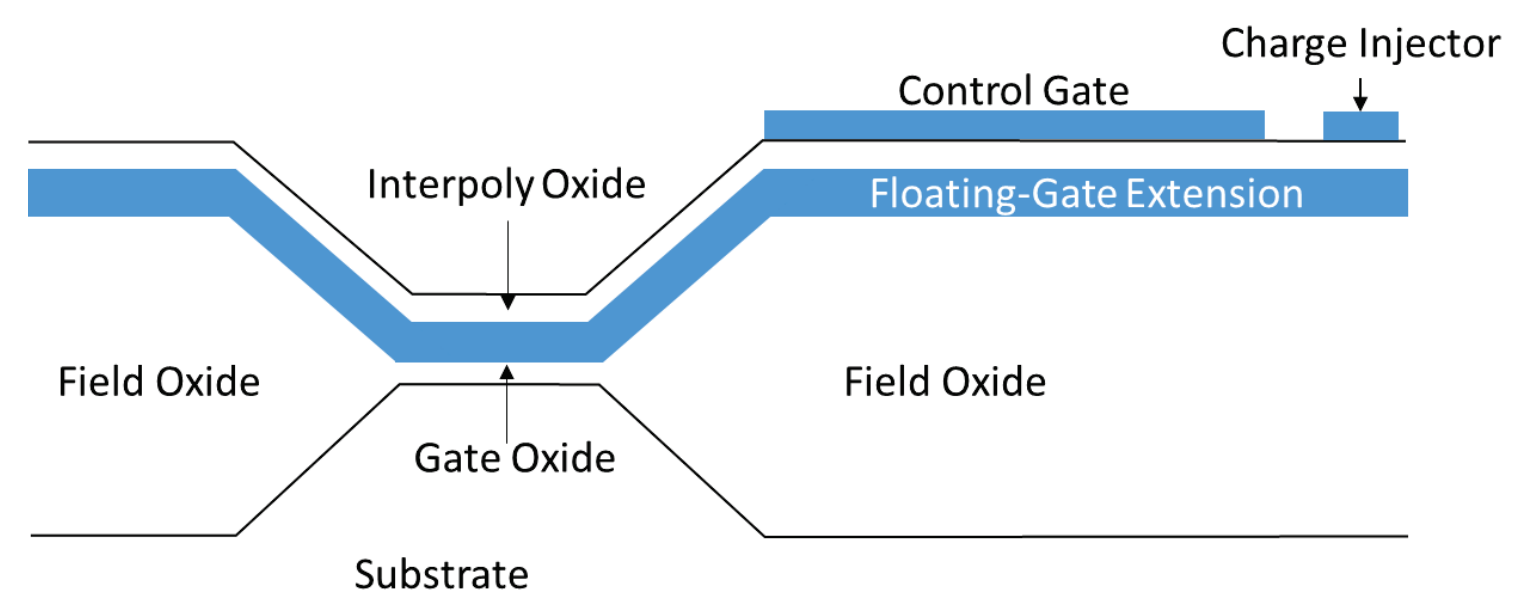

Figure 2.3: Cross section of FG MOSFET device [11]

2. The promise shown by FG MOSFET in dosimetry was picked up by researchers after the initial work of [11]. Consequently, an in-depth theoretical analysis on the operation of this device was presented in 2001 by the team at John Hopkins University [27]. The primary focus of the authors was to study the use of an array based on this technology in a memory block. Using $1.2 \mu \mathrm{m}$ CMOS a $32 \times$ 32 element array was realized and tested for different temperature conditions. The opposite effects of gate capacitance on sensitivity and charge collection efficiency were the main conclusion drawn by the authors. Due to the absence of the paired device, process and temperature effects were seen in the sensors that can be calibrated out with some initial measurements.

3. Continuing on the initial work proposed by his team, Professor G. Tarr came 
up with a much-improved design of FG MOSFET device for the dosimetry circuitry [12]. The aim of the new design was to get rid of the control gate layer that overlaps the floating-gate layer. To accomplish this, a reference transistor was integrated in parallel with the FG MOSFET as shown in Figure 2.4. The subsequent results show an increase of 4 times in the measured sensitivity. Furthermore, the reference transistor also helps in compensating any temperature variations which was previously done by using a paired transistor topology. Nonetheless, using the same CMOS technology as in [11] a superior design of dosimeter circuitry based on FG MOSFET was thus reported.

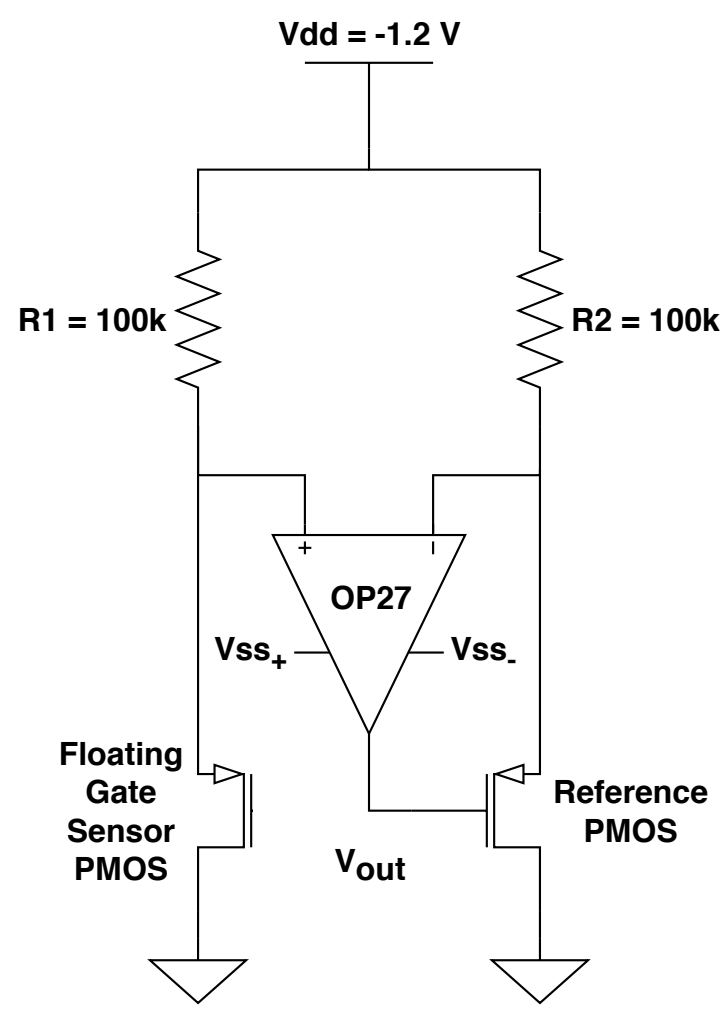

Figure 2.4: Floating-gate dosimeter readout circuitry [12]

4. Between 2012 and 2013 a group of electrical engineers from Spain published 
their research on the design and implementation of FG MOSFET for the application in radiation dosimetry [28-30]. The fundamental operation of these sensor design was the same as initially proposed by other researchers, however they used a different methodology for the detection of the radiation levels. Instead of just measuring the magnitude of the drain current vs the applied radiation level, the authors used a current to frequency converter to generate a square wave at the output. The frequency of the wave corresponds to the strength of the radiation. Greater the intensity of the radiation, greater will be the drain current in the FG MOSFET sensor which will result in a high frequency square wave and vice versa. This technique was applied to gamma radiations and successfully demonstrated. With the lowest detectable value of $2 \mathrm{~Gy}$ (200 rad) the design sensitivity was measured to be $-1.14 \mu \mathrm{A} \mathrm{Gy}^{-1}$ which was reported in terms of frequency in their final publication, i.e. [30].

5. In [31] a preliminary study of MOSFET dosimeters consisting of a floating-gate sensor and a reference gate-connected transistor of identical geometry fabricated in close proximity on the same silicon chip is described. In this research, $0.13 \mu \mathrm{m}$ IBM RF-CMOS technology is used as opposed to previous work done in an older $0.8 \mu \mathrm{m}$ DALSA CMOS technology by the author. The readout circuitry is a unity-gain buffer that reflects the floating-gate voltage on the sensor and it consumes $376.8 \mu \mathrm{W}$ of power. The integrated sensor reaches a sensitivity of $0.4 \mathrm{mV} \mathrm{Gy}^{-1}$ and is targeted for low sensitivity and high radiation exposure applications such as blood sterilization. This thesis will advance the designs reported in [31] while adding other contributions around the topic.

6. A double-poly FGMOS dosimeter fabricated in $0.6 \mu \mathrm{m}$ CMOS technology is presented in [32]. However, the high-power consumption of this design renders 
it unsuitable for the targeted application of dosimetry. Therefore, the design is not of the primary focus for this thesis.

7. One of the recent work around FG MOSFET technology was published in 2015 using a semi floating gate (SFG) as opposed to the conventional technique that was previously reported [33] shown in Figure 2.5. A p-i-n diode was integrated with the transistor just next to SFG in order to control the charge accumulation in it. This p-i-n diode replaces the field oxide present in the conventional FG MOSFETs thus reducing its size considerably. The SFG is charged through the diode in the presence of the gamma radiation. At the same time the diode provides the minimum resistance path to the device during its discharge. Using this technique the authors reported comparable sensitivity levels $\left(65 \mathrm{mV} \mathrm{Gy}^{-1}\right)$ as previously reported work however the device was not realized to verify the simulated results using measurements. In any case the paper laid down the foundation of the work for the SFG technology for radiation dosimetry.

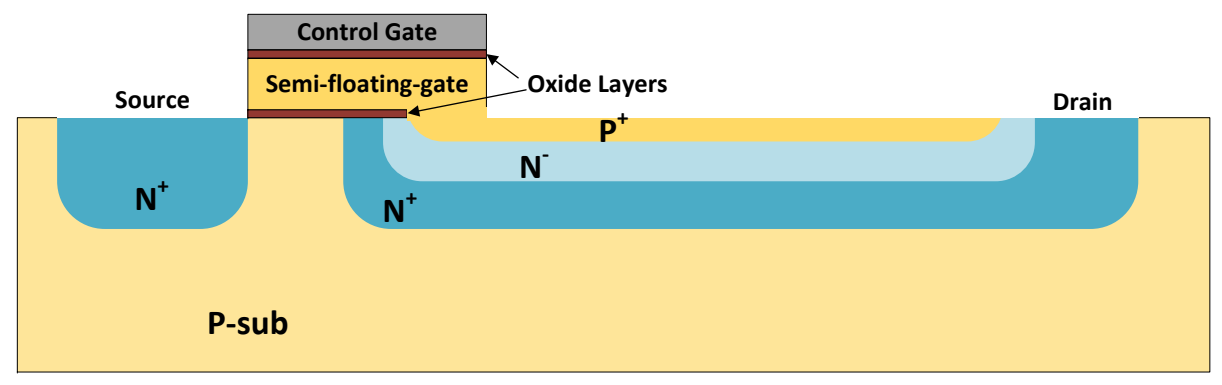

Figure 2.5: Cross section of the Semi-Floating-Gate MOSFET [33]

8. A couple of papers on the FG MOS designs were reported as recent as 2017 [34, 35]. The first one of these concentrates on developing floating gate MOS devices using a non-standard NVM-specific fabrication tailored for memory devices. 
However, the main contribution of this research is to study the effect of doping on the device performance which is not the main focus of this thesis. On the other hand, [35] does report the design of an FG MOSFET device for in vivo dosimetry application but the results thus achieved are preliminary without significant contribution to the state-of-the-art technology.

This section of the thesis summarizes the research area of floating-gate MOSFET dosimeter. Table 2.1 highlights the key performance and design parameters of the literature pertaining to FG MOSFET technology for the said application.

Table 2.1: Floating-gate dosimeter summary of the state of the art

\begin{tabular}{|r|c|c|c|}
\hline References & Technology & Power Consumption & Sensitivity \\
\hline$[11]$ & $1.5 \mu \mathrm{m}$ & $10.4 \mu \mathrm{W}$ & $70 \mathrm{mV} \mathrm{Gy}^{-1}$ \\
\hline 27$]$ & $1.2 \mu \mathrm{m}$ & - & - \\
\hline$[12]$ & $1.5 \mu \mathrm{m}$ & $14.4 \mu \mathrm{W}$ & $300 \mathrm{mV} \mathrm{Gy}^{-1}$ \\
\hline$[30]$ & $0.8 \mu \mathrm{m}$ & $330 \mu \mathrm{W}$ & $-1.14 \mu \mathrm{A} \mathrm{Gy}^{-1}$ \\
\hline$[31]$ & $0.13 \mu \mathrm{m}$ & $376.8 \mu \mathrm{W}$ & $0.4 \mathrm{mV} \mathrm{Gy}^{-1}$ \\
\hline$[32]$ & $0.6 \mu \mathrm{m}$ & - & $278 \mathrm{mV} \mathrm{Gy}^{-1}$ \\
\hline$[33]$ & - & $1.1 \mu \mathrm{W}$ & $65 \mathrm{mV} \mathrm{Gy}^{-1}$ \\
\hline
\end{tabular}

Although FG MOSFET has been explored for decades, no specific publication has been found that would study in a comprehensive manner all the possible modifications to an FG MOS within a specific standard CMOS process for a specific purpose. The extent of exploration within a CMOS technology is usually limited to choosing a MOSFET having a specific area and irradiating it with a range of different sources of ionizing radiation. Within this thesis work, the source of the ionizing radiation is predetermined, hence, it is the aim of this thesis to explore all the possible roads in achieving the best sensitivity while maintaining the lowest power consumed for radiation measurement. For this purpose, the investigation will not be kept limited to using a single device for irradiation testing, rather the research will encompass the 
use of a combination of devices to understand the results.

\subsubsection{Enhancement Techniques for Sensor Performance}

It has been deduced from the comprehensive literature review that most of the work on radiation dosimetry focuses on standard operation of single FG MOSFET device. This allows a lot of room to study the methods by which the figure of merit (FoM) of these sensors can be improved. One major area of emphasis for this thesis is the power consumption of the sensor device. Therefore, some techniques are explored in this thesis to reduce the energy requirements of the sensor while integrating energy harvesting circuits on chip. When the sensors are exposed to the radiations, these circuit blocks can harvest some of the energy which can be used for sensor operation. In this section, some literature on these techniques will be studied.

\section{Non-Floating-Gate MOSFET Dosimeters}

In an attempt to realize extremely low-power radiation dosimetry,an alternative to FG design is also explored in this thesis. The idea is to employ sub-threshold CMOS circuitry for various SPE blocks, and to include a sub-threshold (non-FG MOSFET dosimeter in the SoC). This is a radically new approach. To the best of the author's knowledge, no examples of a sub-threshold MOSFET dosimeter exists in the literature. However, two significant references related to this approach are presented:

1. [36] presents a detailed study of MOSFET behavior in the sub-threshold region of operation in the $0.13 \mu \mathrm{m}$ CMOS IBM technology. High sensitivity of subthreshold CMOS circuits to process variations is addressed.

2. [37] is one of many publications introducing and describing different subthreshold design ideas. However, this reference mostly covers the theoretical 
aspects of the concept without considering the actual realization of the device.

The lack of research on sub-threshold CMOS devices reflects the magnitude of the challenge in this area but at the same time it shows a great opportunity for the designers to explore these devices. The second contribution of this thesis will focus on the realization of sub-threshold CMOS devices and their application for radiation dosimetry.

\section{CMOS Inverter Design}

As discussed in section 2.3.1, almost all the available literature only concentrates on standalone pMOS and nMOS based sensor devices while ignoring the use of inverter for the same application. In 1993, it was for the first time that the application of inverter in radiation dosimetry was proposed [38]. However, this work was preliminary and did not provide comprehensive results to understand the functionality of the device while explaining its operation. No circuit schematics were shown and therefore it is hard to draw a real conclusion from this work. Later on a more comprehensive study on the design and analysis of CMOS inverters for the application of radiation dosimetry was presented in a Master's thesis [39]. The sensitivity of the device when exposed to gamma radiations is shown in Figure 2.6.

Although the results shown here are useful to show the importance of the inverter device in the stated application, the topology employed here does not use the FG concept just like in [38]. Therefore, the effect of radiation on the device is measured with respect to input and output voltage rather than verses the cumulative radiation. As a result, a fair comparison in terms of performance cannot be drawn between these publications and the work undertaken here. 


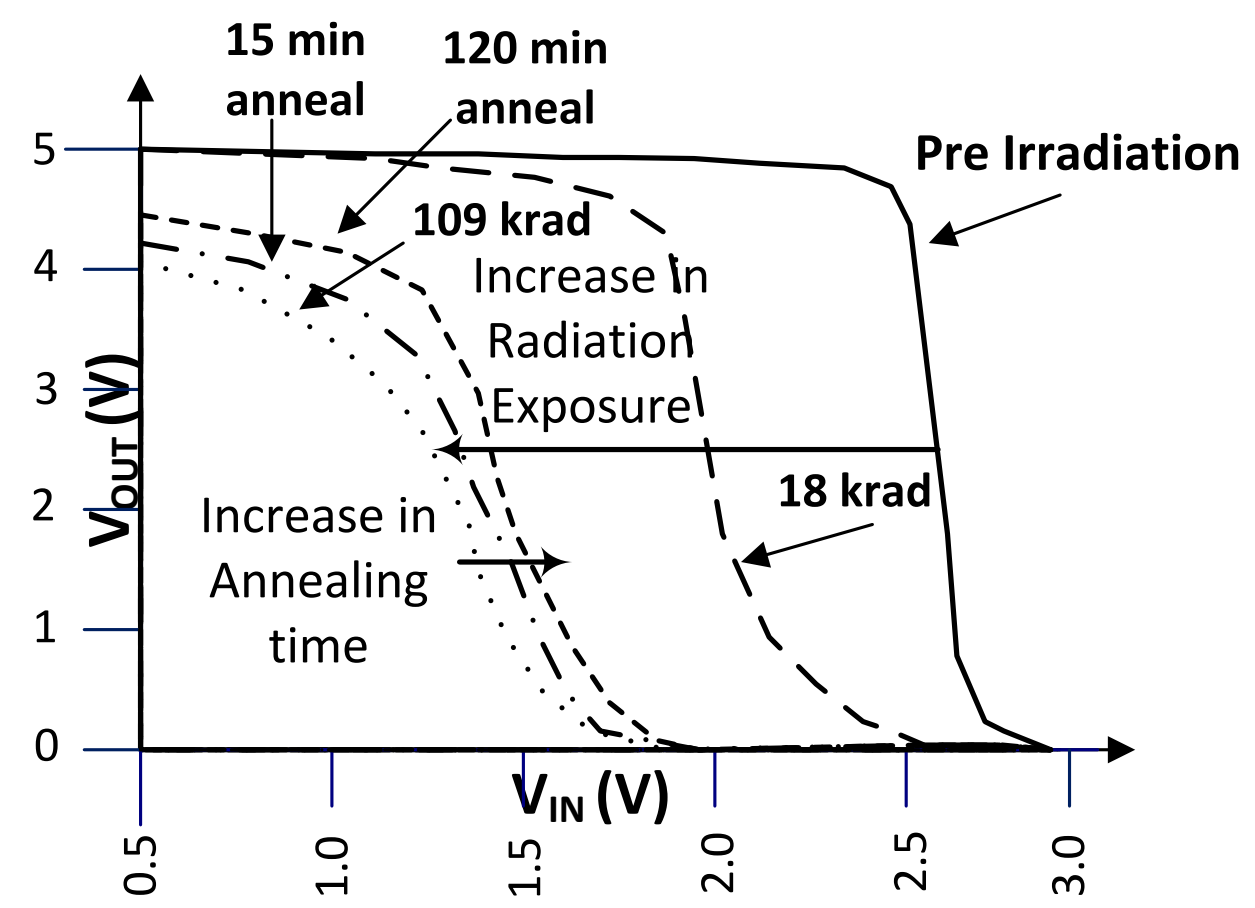

Figure 2.6: Voltage Transfer Characteristics (VTC) of inverter design presented in $[39]$

In a series of publication from 2012 to 2017, a group of researchers from TechnionIsrael Institute of Technology published a series of papers to propose the use of inverter design for the radiation dosimetry based on floating-gate technology [40-42]. The complementary sensor (C-sensor) design concept is depicted in Figure 2.7.

The device is exposed to gamma radiation with a maximum intensity of 150 Gy. A precharge voltage of $4 \mathrm{~V}$ is used before the irradiation. The sensor exhibits a decrease in the gate voltage due to the exposure thus resulting in a sensitivity of $28 \mathrm{mV} \mathrm{Gy}^{-1}$. The sensitivity is shown to improve by using an array of sensors to a value of $100 \mathrm{mV} \mathrm{Gy}^{-1}$. However, the use of array increases the power consumption at the same time. Thus a proof-of-concept for the use of FG MOSFET based inverter design in the radiation dosimetry is successfully proposed for the first time. However, 


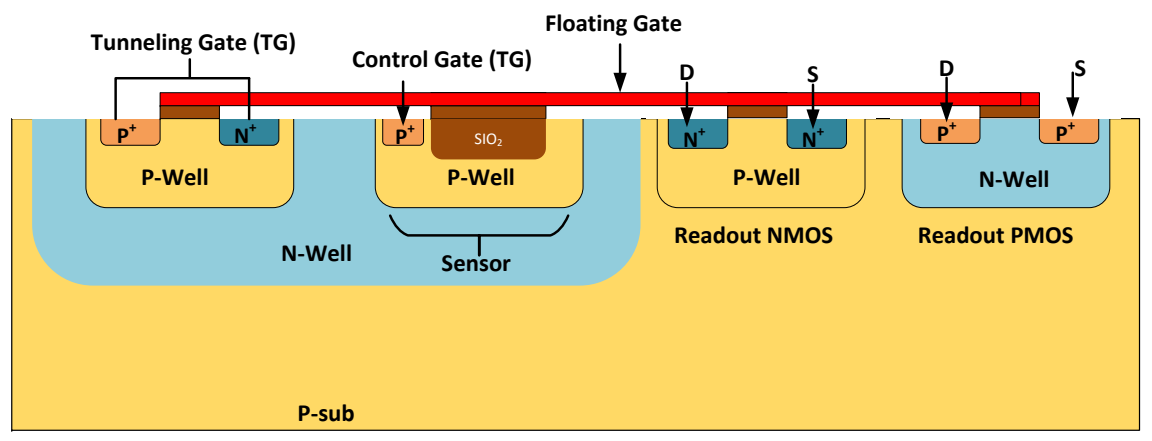

Figure 2.7: FG Inverter design realized in 180 nm CMOS technology [42]

the power consumption of these sensor devices is similar to the ones reported before. In fact, the use of an array instead of a single device does improve the sensitivity but at the expense of the power consumed. In this thesis, the idea of using CMOS inverter design is studied to improve the power consumption while providing comparable sensitivity values. Figure 2.8 shows the radiation exposure response of the CMOS inverter where $V_{t}$ is the switching point of the inverter which gets modified by irradiation.

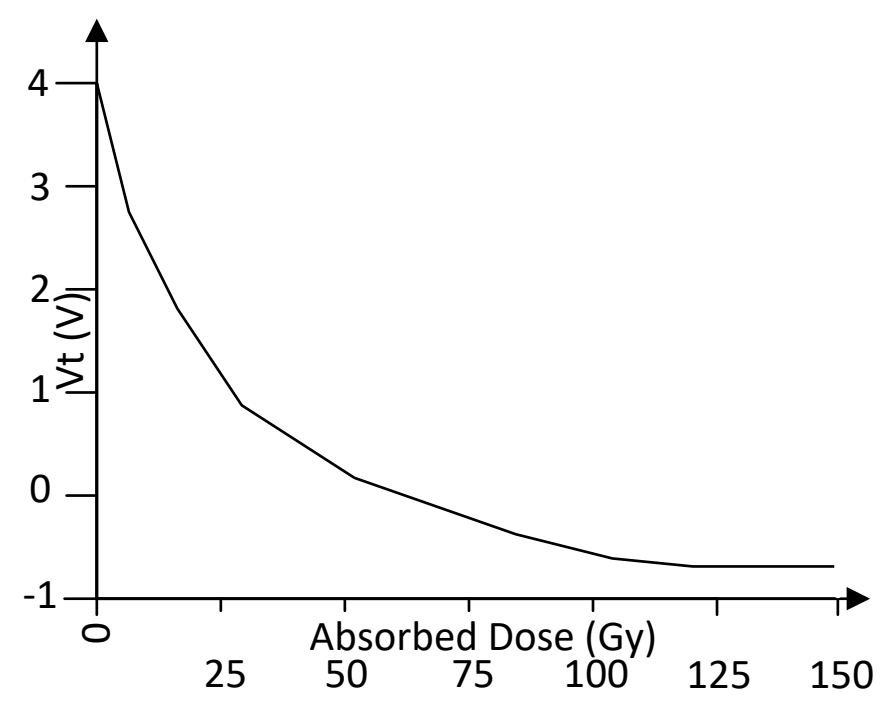

Figure 2.8: Voltage response of FG-CMOS inverter design to gamma radiation from CLINAC; $V_{t}$ is the switching voltage of the inverter [42] 


\section{Self-Powered Radiation Dosimeters}

Almost all the solutions for radiation dosimetry rely on external power source for their operation. There is hardly any work done in this direction specially when the sensor is based is on MOSFET technology. This opens a new door for proposing energy harvesting circuits alongside the sensor device thus introducing the concept of batteryless radiation sensors. Although not completely relevant, a group from Politecnico di Torino proposed an ultraviolet (UV) radiation sensor with wireless capabilities to communicate the collected data in 2013 with integrated energy harvesting blocks [43]. Two different techniques were employed to provide the energy required to operate the sensor: solar harvester and thermal harvester. Without going deep into the details, the problem here is the use of sources other than radiation itself to power the device renders this reference immaterial for this work while providing a basis of for the Self-Powered Radiation Dosimeter. A comparatively related article on the topic was published last year (i.e., 2017) in IEEE Sensors Conference [44]. The design concept for this work is depicted in Figure 2.9. JFET based Schottky diodes are used for energy harvesting purposing. The device is supposed to be employed in an X-ray dosimeter. By exposing the device to different strengths of radiations the authors showed generation of voltage across the diode which can in turn be used to supply power to the sensor integrated on the same board. Using radiation power levels of $28 \mu \mathrm{W} \mathrm{cm}^{-2}$ the diode was shown to generate voltage in the range of $0.7 \mathrm{~V}$. This value cannot operate the sensor on its own and therefore requires a Boost converter circuitry to amplify the voltage. This is a unique concept whereby the sensor device is supposed to work on its own without any external source. However, the design is in its preliminary stage with minimum information on the design and integration. The sensor design is to be based on FET technology but the details on the circuit

or the device implementation are completely absent. Thus, it is hard to conclude 


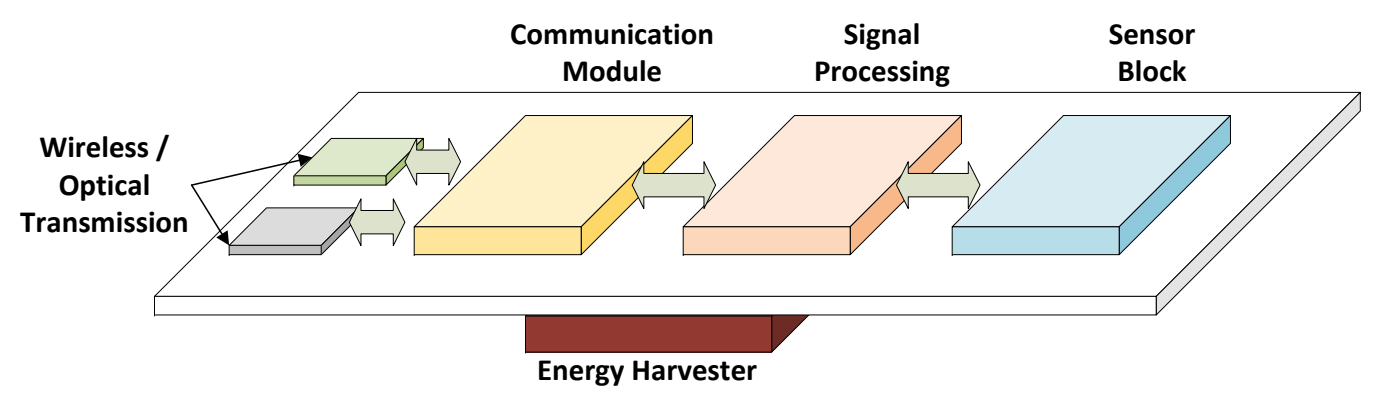

Figure 2.9: System Concept proposed for self-powered X-ray dosimeter [44]

the figure of merit of this design. Keeping in view the lack of literature in the field of energy harvesting in radiation dosimetry there is a clear need of investigation in this direction. In this thesis energy harvesting using the same radiation source whose intensity is to be measured will be used to provide the power required by the sensor. This will be the first ever proof of concept demonstration towards an autonomous radiation dosimeter sensor independent of external power source.

\subsubsection{Non-Volatile Memory (NVM) Designs for RFID Ap- plications}

The wireless dosimeter system proposed in this work requires the realization of a one-time programmable (OTP) memory design with tailored characteristics and performance parameters. There has been considerable work done on this topic over the past few decades. However, here a brief summary of designs that are most relevant to this work will be provided. In 2009, researchers from ST Microelectronics in France published a comprehensive summary on the design of non-volatile one-time and multiple time programmable memory [45]. Using $65 \mathrm{~nm}$ and $45 \mathrm{~nm}$ CMOS technologies a detailed performance analysis outlining the density comparison is presented. From the literature present at the time the paper concludes that the metal fuse, poly-fuse 
and anti-fuse are all good candidates for the OTP memory implementation as compared to other technologies out there. The intended design prefers metal gate to polysilicon gates on the basis of fabrication complexities for fuse solution. On the other hand, anti-fuse structures have been fabricated and measured as they provide the best trade off in the current CMOS technology and can be further extended to $32 \mathrm{~nm}$ and beyond. This work is an excellent platform for the research carried out on memory design using the conventional CMOS technology. Previously, memory cell based on the eFuse (electronic fuse) was published in Microelectronics Journal [46]. A comprehensive performance analysis on the read/write characteristics of the proposed design is presented. A scanning electron microscopy (SEM) image of the eFuse device in the blown and unblown scenarios is added here in Figure 2.18. The work also outlines the resistance variation that occur in the device due to the difference in the current flow before and after programming. The relevance of this work with the thesis is the utilization of $0.13 \mu \mathrm{m}$ RF-CMOS technology for the memory implementation. However, the circuit blocks suggested in [46] are different from the ones used here. Further to [46], another articles entails the use of eFuse devices for programmable memory solution. Here the authors go one step forward by introducing the concept of on-chip self-repair using eFuse solution. To accomplish this compressed repair data is programmed inside the fuse itself at the conclusion of the test. Although the concept is reasonably interesting and describes the system level implementation, it does not go into the details of the integrated circuits that are realized to get the desired results. Since the focus of this work is on the device level, it is hard to draw a fair comparison between the performance of the two concepts. Still, the concept of self-repair is something quite novel and hence included in this section. Papers [47] and [48] illustrate other options for OTP memory cell architecture, namely the deferential eFuse cells. 
A good summary of non-volatile one-time and multiple-time programmable memory design has been presented in [45]. Furthermore, [46] provides a description of the eFuse memory cell read/write characteristic and resistance changes due to current flow before and after programming. The significance of this publication is in that it introduces the eFuse cell in the same CMOS technology this thesis work employs. The read/write (sense/program) circuit blocks suggested in that paper is not utilized in this thesis. In terms of memory architecture, the work by [49] gives an overall system description of an eFuse memory block without getting into any design details. Papers by $[50,51]$ illustrate other options for OTP memory cell architecture, namely the differential eFuse cells.

Floating-gate memories are specific memories which can be used as OTP. Thanks to their floating gate which can store charges, they can be written and erased many times [52]. A single polysilicon floating gate is presented in [53] that is standard CMOS process compatible. A coupling capacitor is associated to a tunneling capacitor for operations of the bitcell. Programming is performed by capacitive coupling and erasing is based on Fowler-Nordheim mechanism. This memory structure depends on electric charge accumulation on the floating gate which shifts the threshold voltage of the MOSFET. This shift in $V_{t h}$ (threshold voltage) can be sensed and interpreted as a bit value of 1 or 0 . However, this architecture is not suitable for NVM design in the case of this research work since the ionizing radiation will deplete the charge on the FG, which causes an erroneous bit read.

Other OTPs exist such as phase change memory (PCM) [54-56], semiconductoroxide-nitride-oxide-semiconductor (SONOS) memories $[57,58]$, and resistive random access memory (R-RAM or ReRAM) [59]. Implementation of these memory solutions require extra processsing steps and layers which are not only more costly, but also not 
generally provided within IC design kits. While the above-mentioned list of memory solutions does not represent all the different kinds of memory available, it can be concluded that the viable choices are limited to either the electronic fuse (eFuse) or the antifuse structures.

\section{Antifuse Architecture and Related Work}

Most antifuse structures rely on intentional permanent damage caused by applying a large voltage to the gate oxide of a MOSFET as a means to write a bit value. Figure 2.10 illustrates an antifuse memory unit and a possible write circuitry [60, 61]. A programming voltage $V_{P P}$ is applied to either the anode or the cathode (the gate or the shorted drain-source regions of the MOSCAP) depending on the design, causing the gate oxide to break and essentially short out. Generally speaking, an antifuse architecture for a bitcell always involves a MOSCAP and an access transistor. The programming voltage applies an electric field across the oxide, stressing it and causing the injection of carriers through the oxide which in turn creates traps within it. Once a critical density of traps is created, a conductive path is formed which allows current to pass through, essentially breaking down the oxide. There exist two different breakdown phenomena, 1) the quasibreakdown which results from the connection of many conductive spots within the oxide and 2) the complete breakdown. In complete breakdown, as current flow increases, the local heating effect leads to a current runaway which creates a cylindrical path between the polysilicon gate and the silicon substrate. The difference between the two phenomena is extensively described in $[62,63]$ and shown in Figure 2.11. The two breakdown phenomena cause a large variation in resistance in the channel created, hence different current characteristics. Antifuse cells are classified into 4 different categories: 

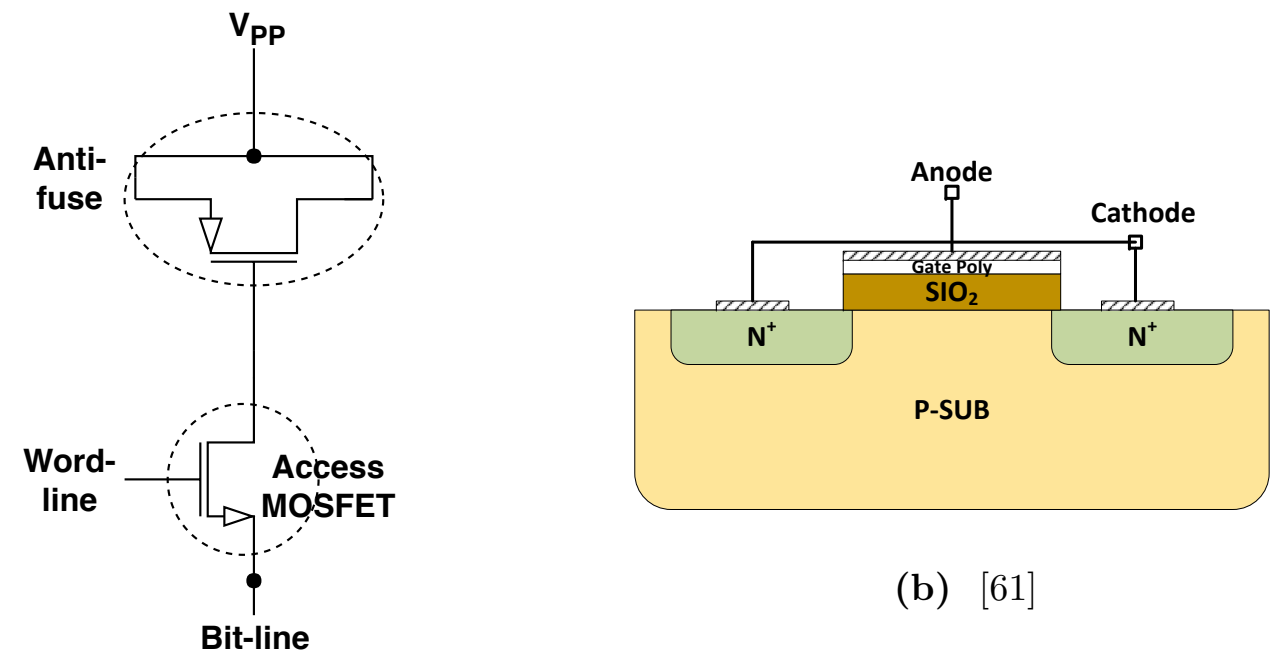

(b) $[61]$

(a) $[60]$

Figure 2.10: Antifuse memory unit, (a) possible write circuit, (b) unit structure.

1. 0T-1C antifuse This architecture features a parallel plate capacitor in series with an access diode to "write" (burn) and read the capacitor state. It is also called Diode Programmable Read Only Memory (DPROM) [64]. A sample of the cell structure is presented in Figure 2.12.

This memory structure requires large programming voltages in the range of $\sim 13 \mathrm{~V}$. It also requires special fabrication technology, hence becoming very costly and less available commercially. Moreover, due to its high programming voltage, neighboring bitcells are also stressed during the application of the high voltage, hence reliability becomes an issue. Due to the complexities mentioned, this type of NVM is not suitable as a system-on-chip solution.

2. 1T-1C antifuse This structure is composed of an access transistor and a capacitor. An example is presented in [65] and shown in Figure 2.13. Similar to the first architecture, a programming voltage is applied to the capacitor and another voltage is applied to the access transistor to create a high potential 

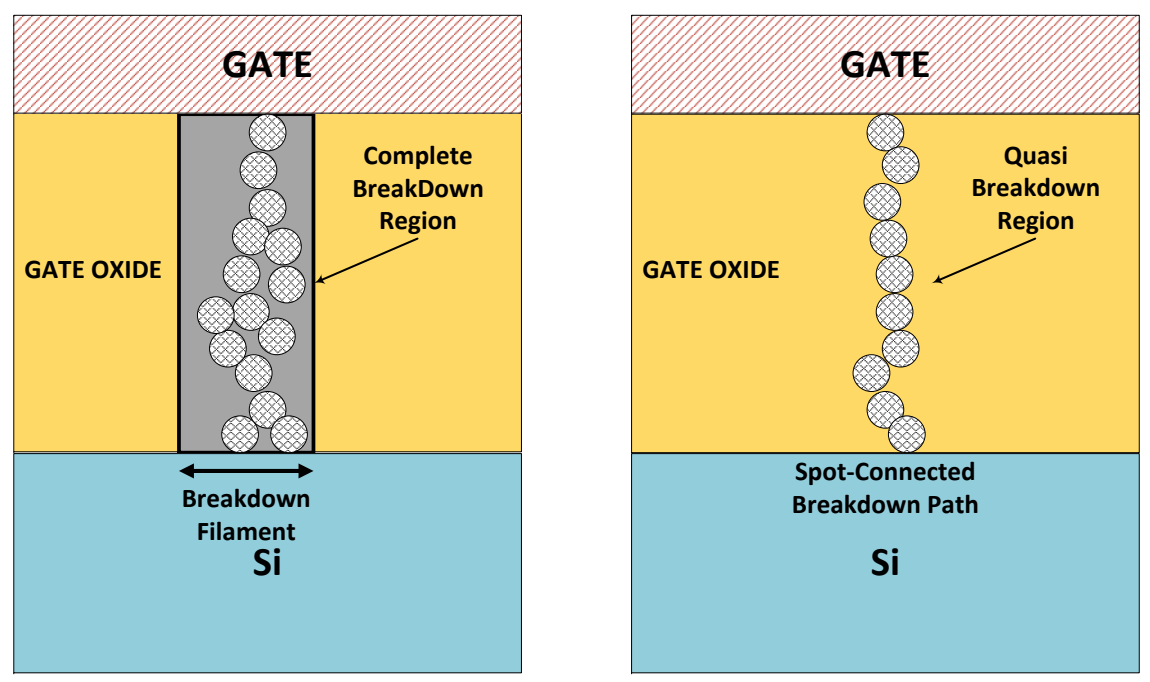

Figure 2.11: Illustration of the difference between a complete breakdown and a quasibreakdown of the gate oxide [62].

difference between the two plates of the capacitor and break its oxide. A reliability issue arises from the fact that once the capacitor oxide breaks, the drain of the transistor experiences a large voltage which could adversely affect it or even break the transistor gate oxide at the drain junction rendering it inaccessible. Moreover, it is necessary to use a thick-gate high voltage transistor as an access transistor to increase the robustness of the combined structure against high programming voltages. This need increases the size of a bitcell which in turn affects density.

3. Drift transistor antifuse To overcome the inherent drain junction stress mentioned above, another architecture was developed. This custom design utilizes an $\mathrm{N}$-well instead of the $\mathrm{N}^{+}$drain diffusion in order to minimize drain stress under the writing operation. The reported process uses similar gate oxide thickness of $\sim 5 \mathrm{~nm}$. The cross section of the drift antifuse structure is presented in 


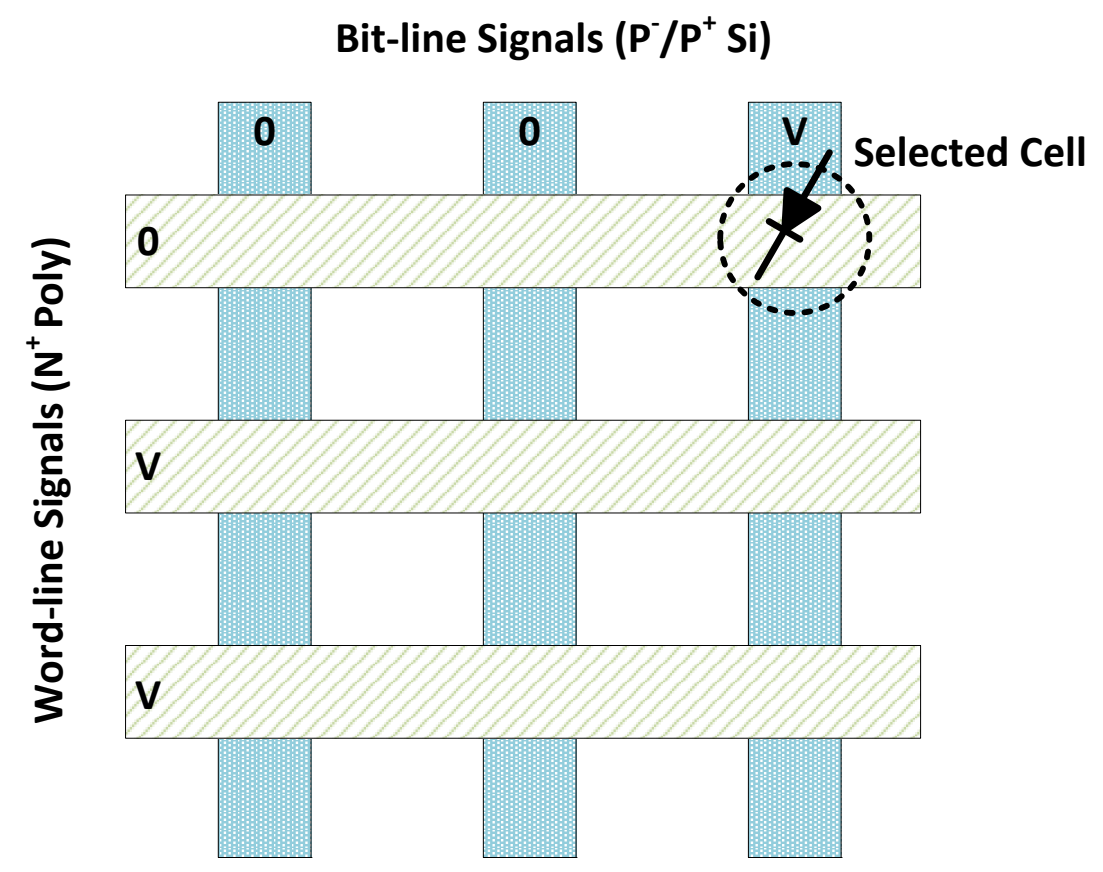

Figure 2.12: A diode antifuse is programmed at the crossing of the selected bit and wordline. [64]

Figure 2.14 [66]. This design requires that the designer be able to select between $\mathrm{N}^{+}$or $\mathrm{P}^{+}$doping which is not a choice in our technology. Also, the drift NMOS is sensitive to N-Well mask misalignment, i.e. the L, Olap (overlap layer), $L_{n \text {-well. }}$. This makes the layout for such design a challenge. Also as explained by the author, other considerations need to be taken into account in order to overcome capacitor gate oxide stress in addition to the access transistor (NMOS) design. For non-selected cells, a larger capacitor and smaller access transistor would reduce the stress, whereas, for selected cells to be written to, the opposite is true. Therefore, the design becomes an iteration of experimental evaluations in order to decide which exact dimensions would satisfy reliability, power consumption, and area occupied by each capacitor cell. In this design, a programming voltage of more than $8 \mathrm{~V}$ is required to write to a capacitor which 


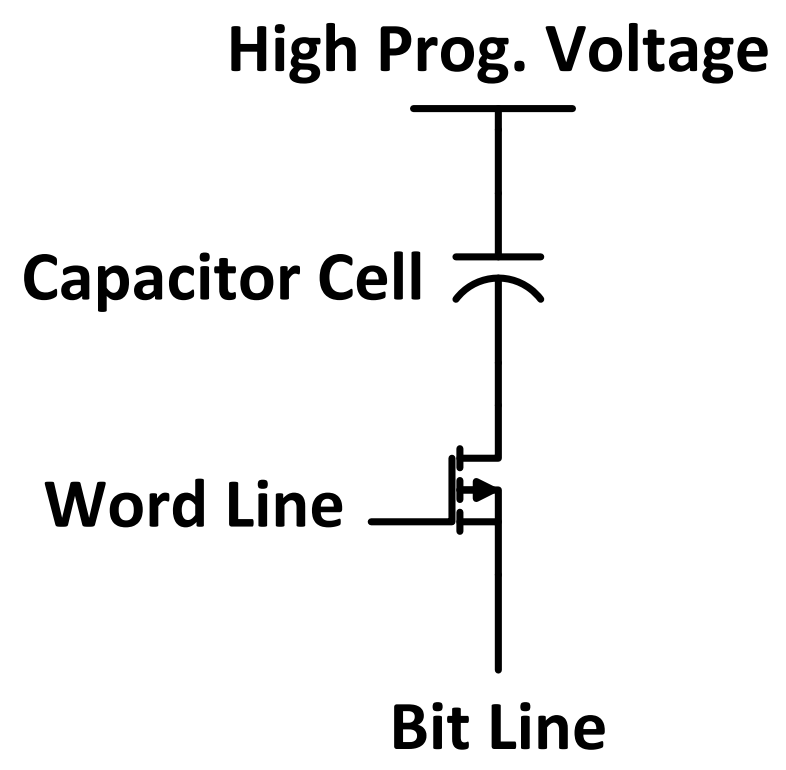

Figure 2.13: Capacitor and access transistor bitcell structure. A high voltage HV is applied to the capacitor's top plate while another voltage is applied to the access transistor in order to program the cell [65].

also worsens the reliability issue. A circuit representation of the write operation is given in Figure 2.15 where the author emphasizes on the gate oxide stress exerted on the non-selected cell.

4. 3T antifuse To mitigate the persisting issue of drain stress caused by the oxide breakdown, another solution has been presented in $[45,67-70]$ and shown in Figure 2.16. While this approach reduces the drain stress on the lower transistors in the cascade chain, it still leaves the adjacent transistor to the bitcell vulnerable to drain strain. In addition, having a long chain of transistors adds to the logic and layout complexity and consumes more chip area. Body biasing also becomes an issue which could increase the transistor threshold voltages which reduce the amount of current passing through the transistors both in read and write modes. This becomes a problem in read operation sensitivity, 


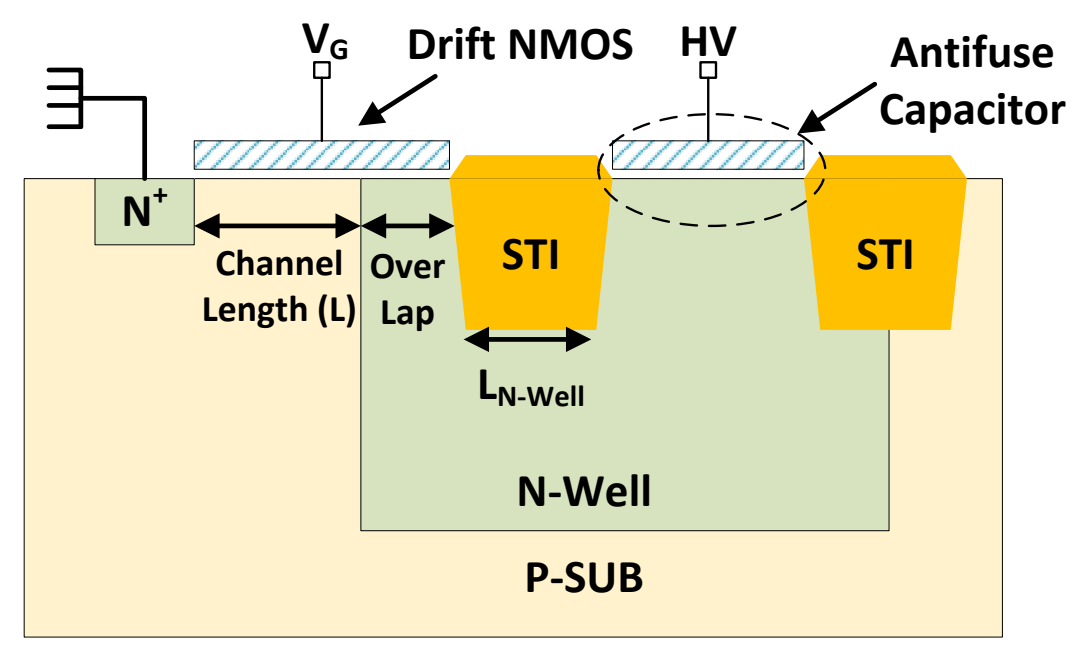

Figure 2.14: Cross section of the drift antifuse cell. [66]

so the designer needs to increase the operating voltage to compensate.

To summarize, in addition to the reliability issues such as high voltage programming, gate oxide thickness and other property mismatch, soft or hard breakdown and the resulting resistance variation among programmed bits, read current spread due to the latter phenomena, and layout dependent read/write yield in bitcells, another very important issue is the effect of neighboring cells on each other in both read and write operations. This issue is introduced in all the cited publications as the programming window. The programming window describes the voltage range suitable for programming a capacitor while avoiding damage to the neighboring capacitors not meant to be programmed as shown in Figure 2.17. This voltage range can be a source of reliability issue since due to process variations, a cell could tolerate higher or lower voltages, hence limiting the range.

Next, eFuse structure and related publications are studied. 


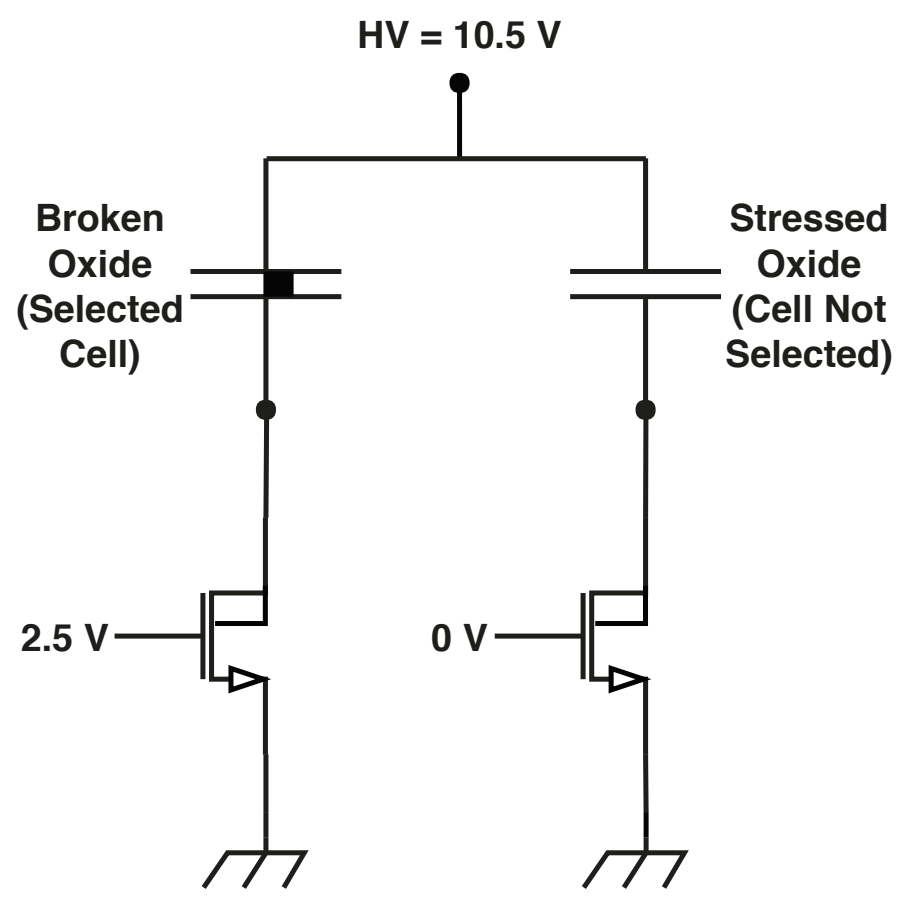

Figure 2.15: Circuit representation of a selected and a non-selected bitcell during programming using a drift NMOS architecture [66].

\section{eFuse Structure and Related Work}

Figure 2.18 shows a blown and an unblown eFuse structure [71].

An eFuse structure consists of a thin polysilicon layer connected between two ports (terminals) often noted as anode and cathode. The silicide layer covering the polysilicon and the polysilicon layer itself are subject to high current flow which permanently damages the layer. This physical change in the structure causes a resistance change between the two ports. This change can be compared to a reference device (or voltage) to determine whether a fuse has been damaged (blown or written) or intact (unblown). The first ever eFuse was reported in [72] presented by IBM Microelectronics in 2002. In this paper, in addition to the physical structure of the eFuse, the authors present their understanding of the phenomenon based on which the eFuse programming happens. Electromigration in the silicide layer is mentioned as the principal 


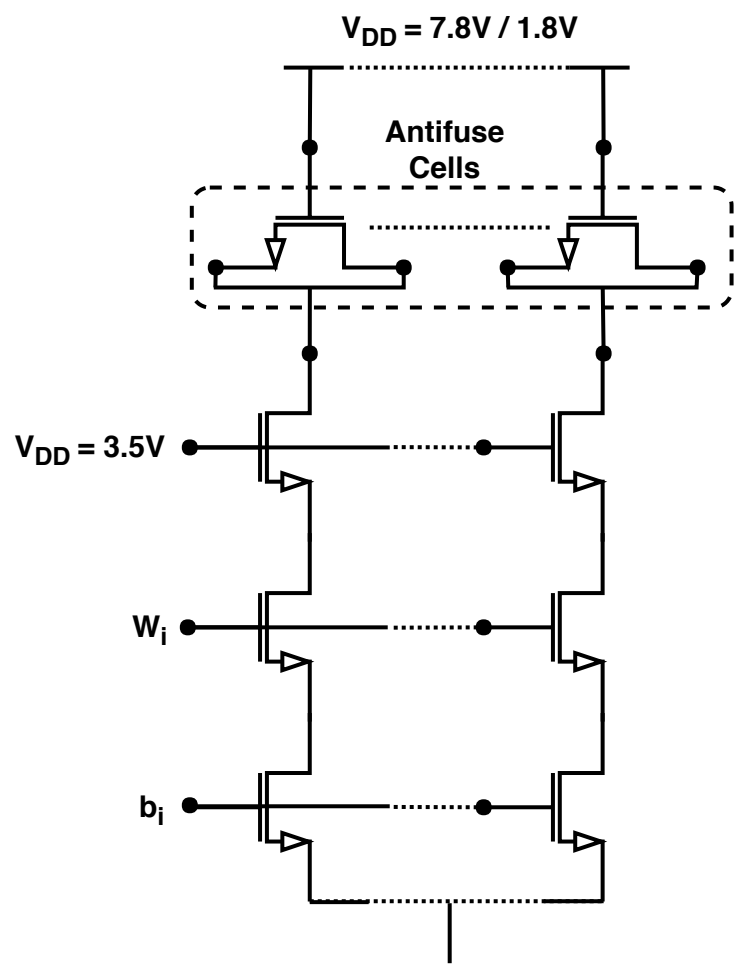

(a) $[70]$

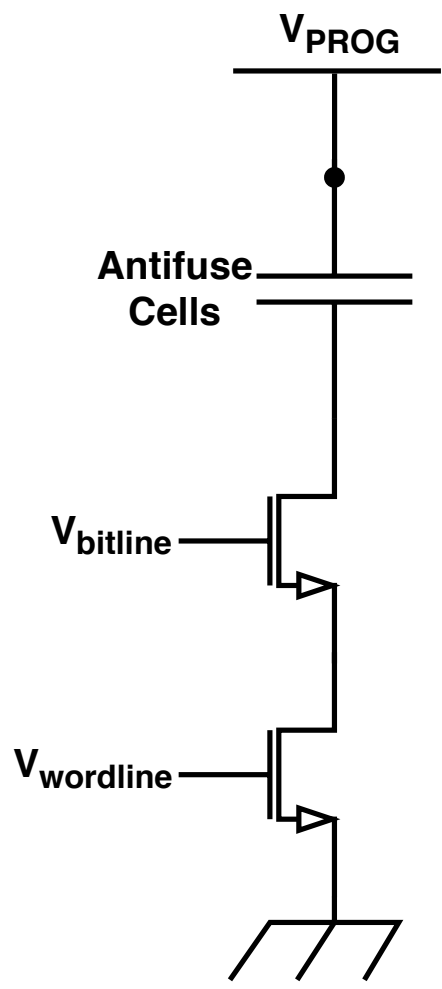

(b) $[45]$

Figure 2.16: Antifuse bitcell architectures; access transistor cascoded with other transistors to lower the drain voltage.

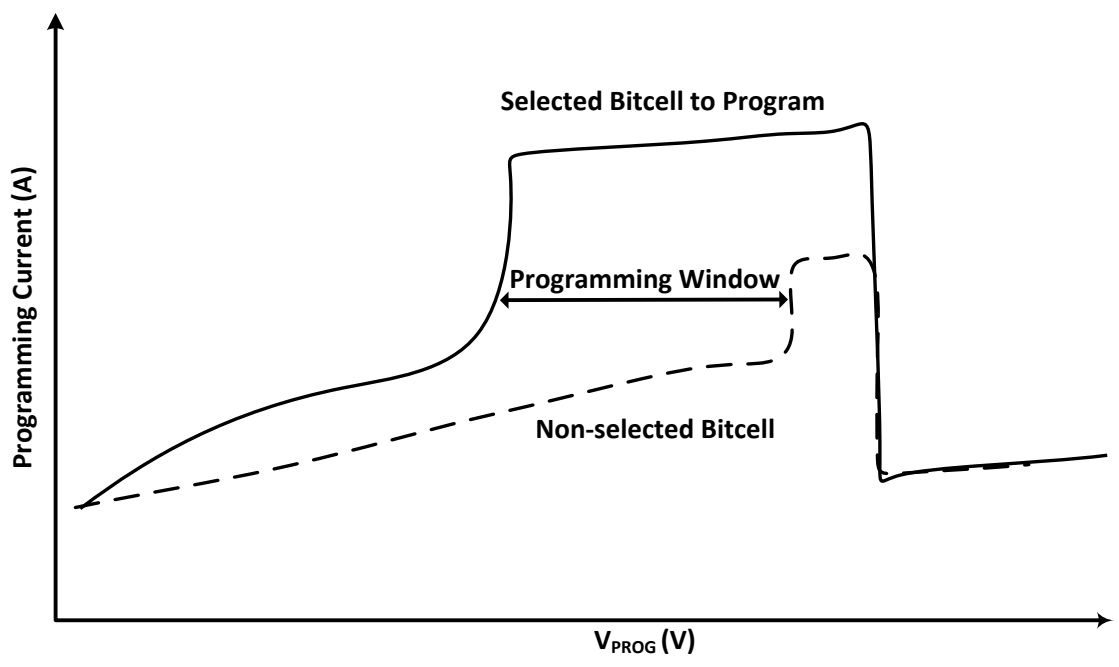

Figure 2.17: Static programming curves for a $1 \mathrm{~T}-1 \mathrm{C}$ antifuse with thin or thick oxide access transistor describing "programming window" [45]. 


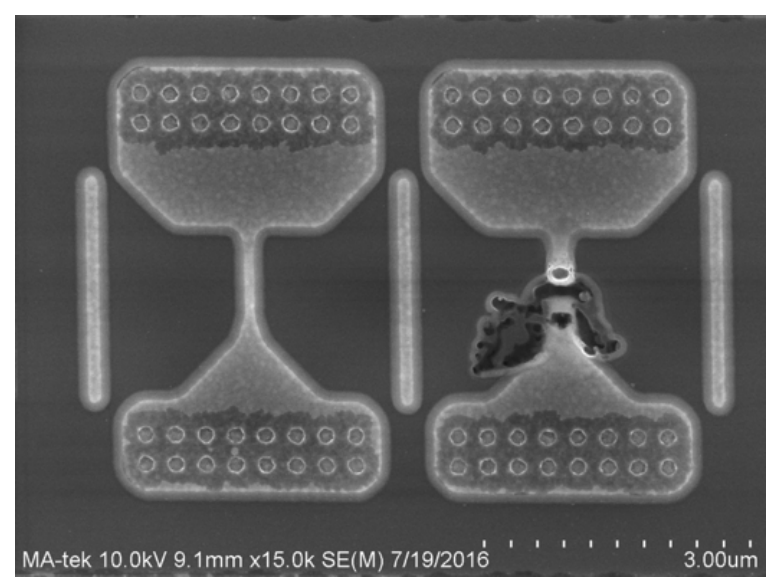

Figure 2.18: A blown (right) and an unblown (left) eFuse structure [71]

effect responsible. Other publications such as $[73,74]$ also emphasize electromigration as the dominant effect in eFuse programming. Other phenomena proposed that could play a role in the breaking of the fuse are explained in [45]. What is common in all the publications is the fact that a large current typically greater than $10 \mathrm{~mA}$ is necessary in order to permanently damage (program) the eFuse. The preprogrammed resistance of the fuse is $\sim 50-200 \Omega$, whereas post-programmed fuses can have resistances $\geq 4 \mathrm{k} \Omega[46,75,76]$.

There are a few memory architectures that have been proposed for programming and reading of the efuse. The reading block can be grouped into three main categories:

1. Single-ended sensing scheme where a cross-coupled inverter pair latch is preset to the 1 state (blown fuse). If the fuse is intact, its resistance is low enough to pull the " 1 " down to a "0". If the fuse is blown, its resistance is high enough to maintain the "1" $[46,47,77]$. An example illustrating single-ended sensing in Figure 2.19 has been presented in [47].

While a simple sensing mechanism, it is not used often due to resistance variations in both blown and unblown efuses which could lead to incorrect sensing. 


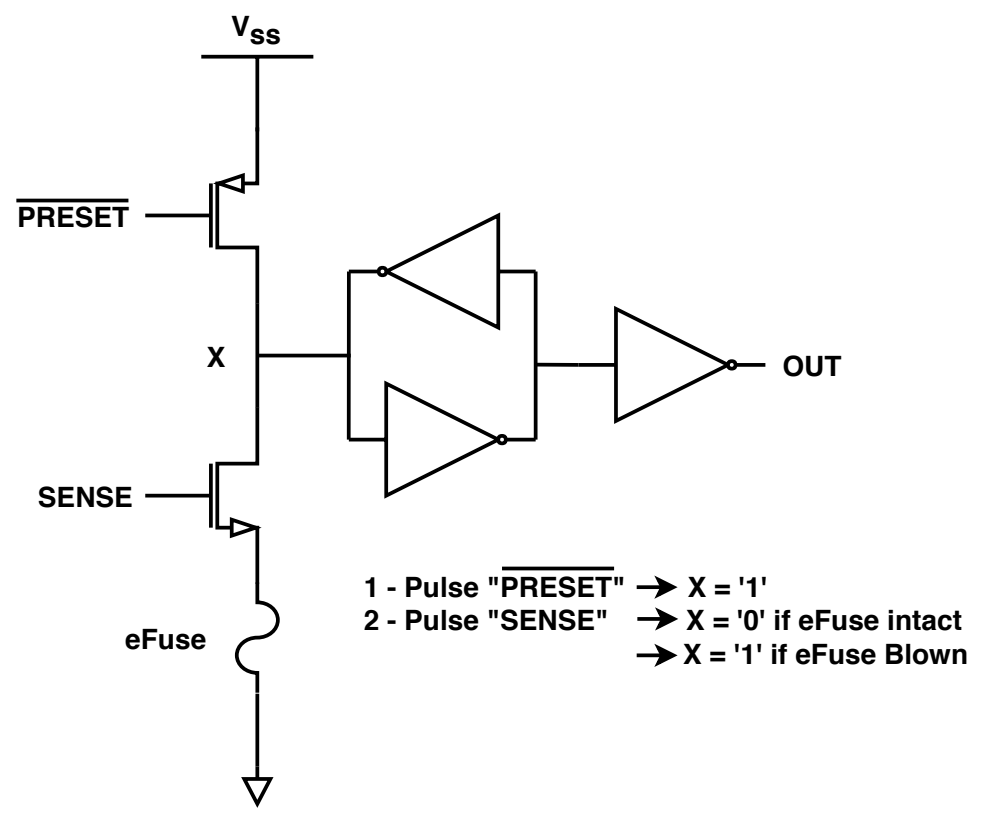

Figure 2.19: Single-ended eFuse sensing [47].

Also, variations in the threshold voltage of latch PMOS transistor could decrease its current drive leading to instability. Moreover, sensing either a " 1 " or a "0" creates a static current path. Hence, power consumption becomes dependent on the "sense" signal duration. Other reliability issues have been noted in $[47]$.

2. Differential eFuse sensing that helps increase the sensitivity of the sensing circuit in comparison to the single ended design topology $[47,48,50]$. In other words, the differential sensing topology reduces the margin between the sense trip point and the extreme fuse values in the case of blown and unblown fuses. The differential sensing is divided into three subcategories:

(a) True/Complement 2 fuse scheme This scheme, shown in Figure 2.20 utilizes two eFuse cells for each memory bit. There is no default condition for this architecture. 


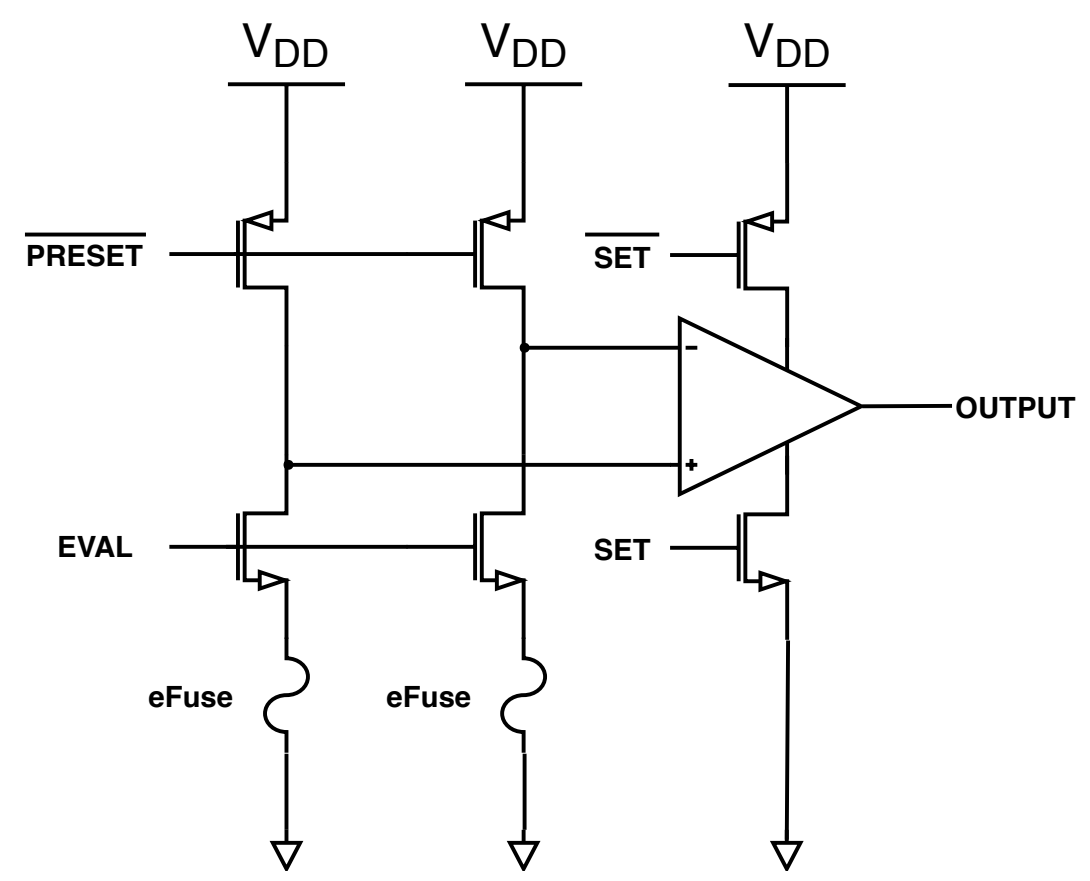

Figure 2.20: Differential True/Complement eFuse sensing circuit [47]

In programming each cell, both eFuses need to be programmed whether a " 1 " is being written or a " 0 ". The advantage is its robust fuse sensing solution since margin to the programmed fuse only is required. However, this topology offers a one-pass programming solution, meaning modifications to the " 0 "s cannot be done once programmed. Also, utilizing 2 eFuses for a cell requires twice the area considering the already large programming transistors and metal routing to handle the large current. It also introduces minor additional logic complexity to both programming and sensing blocks.

(b) Local reference; 1 reference, 1 fuse cell where a reference is replaced with an eFuse in the cell. This reference could be a polysilicon resistor 2 or 3x the unblown eFuse value. An eFuse resistance value below this reference sets the latch to a logic "0" whereas a resistance value above the reference 
sets the latch to a logic " 1 ". While this approach is mentioned in [47], to the best of the author's knowledge, there is no mention of such cell topology in other publications. This design topology also requires double the area of a single eFuse cell as well as careful choice of the reference resistors. Due to process variations, the reference resistors could have a large variation with respect to each other, hence increasing the chance of an error in bit sensing.

(c) Shared reference; 1 reference, many fuse cells in which the reference resistor is shared among many eFuses. This scheme benefits from increased sensitivity and robustness in addition to smaller area. It is also immune to process variation since all fuses are compared to only one reference. An example is presented in Figure 2.21. Other examples of the differential sensing topology are presented in Figures 2.22 and 2.23. These topologies are designed mainly for verification purposes.

Implementing such sensing circuits would require large chip areas considering the number of on-chip resistors. Also, it adds to the complexity and further power consumption of the chip which renders these topologies inefficient in a low power application such as the radiation detector chip ID. These designs require also at least 7 different signals applied in order to sense the bit cell value which makes signal timing a significant design concern. 


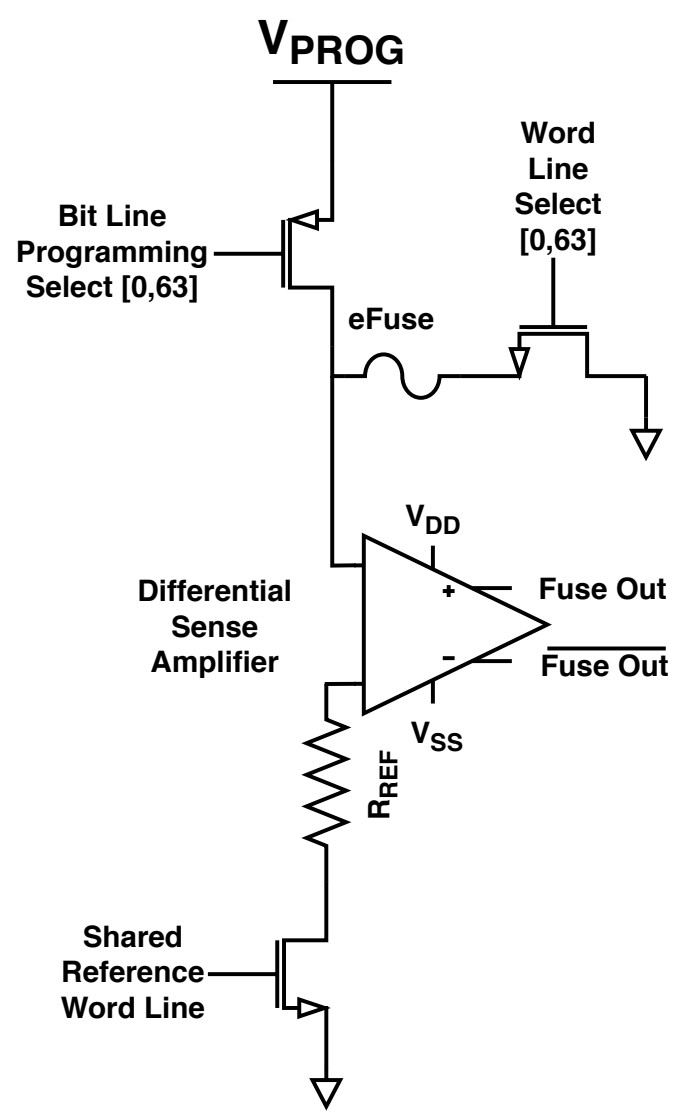

Figure 2.21: Shared reference sensing circuit [47]

\subsection{Conclusion}

A comprehensive review of the designs of FG-MOSFET dosimeters, integrated energy harvesting techniques and non-volatile memory has been reported in this chapter. It has been clearly indicated where the relevant technologies stand as of today. All these publications have their novelties, showing improved performance. However, there are still performance parameters that can be further investigated and enhanced. At the same time, hardly any work has been reported on the topic of energy harvesting for radiation dosimetry. Within this context, the objectives defined for this thesis will be tackled in the subsequent chapters. 


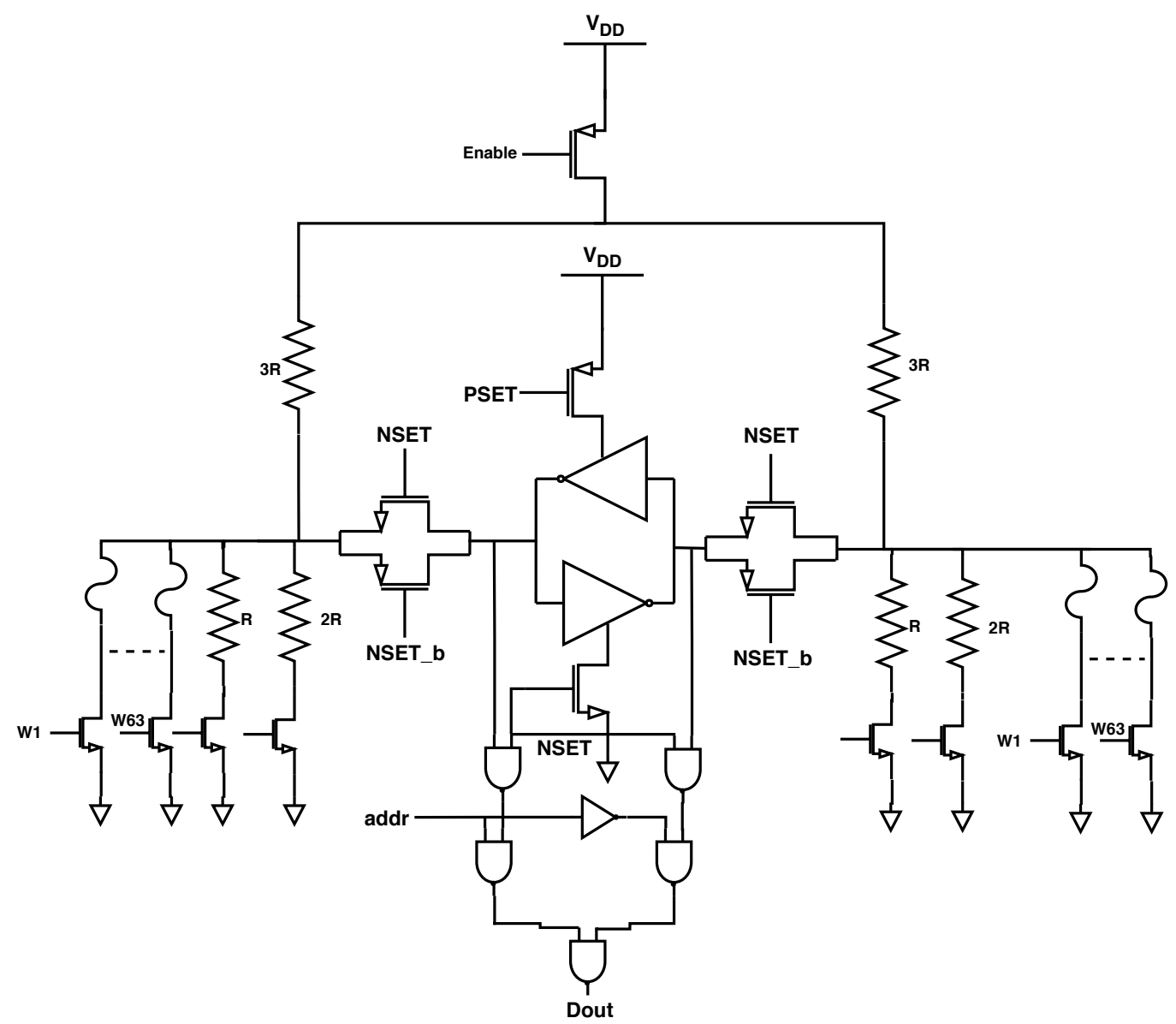

Figure 2.22: A differential sense amplifier with programmable reference [78]

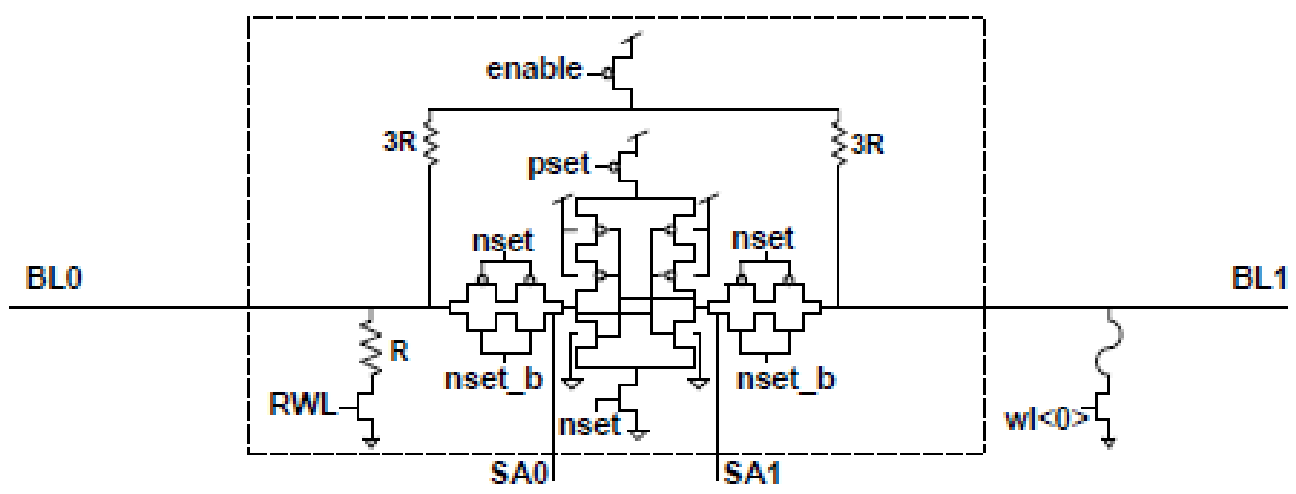

Figure 2.23: A differential sense amplifier with programmable reference [78] 


\section{Chapter 3}

\section{Floating-Gate MOSFET Dosimeter Design}

\subsection{Introduction}

In pursuit of the first thesis objective (design and characterization of an FG-MOSFET dosimeter), the following specific objectives will be followed in this chapter:

3.1. Parametric study of design parameters to define optimum configuration for sensor linearity, sensitivity, power consumption, and dosimetric range.

3.2. Fabrication and experimental validation of the designs considered in 3.1.

3.3. Experimental determination of the best pulse shape, duration, and height for charging and discharging of the FG sensor to allow synthesis of a future automatic charging system that could be implemented on chip. 


\subsection{Principles of Operation: Radiation Sensing \& Programming}

The principles of ionizing radiation sensing by floating-gate MOS devices have been extensively explained by many publications, [11, 12, 16, 29,31,79,80] to name a few. The simplified precharging and sensing of a floating-gate MOS sensor is depicted in Figure 3.1. Initially the floating gate is precharged with excess charge using a series of negative pulses of $\approx-7 \mathrm{~V}$ from an external source. Ionizing irradiation creates electron-hole pair inside the thick oxide layer formed under the floating-gate extension. The generated electrons and holes are separated from each other due to the electric field that exists within the deep oxide formation. The electrons from the floating gate recombine with the generated holes that are attracted to them. Another mechanism is that the excess electrons on the floating gate receive enough energy from the incident X-ray photons so that they could escape the floating gate. Moreover, the dielectric layer on top of and around the floating-gate extension contributes holes generated by ionizing radiation, which in turn recombine with the excess electrons. These three main mechanisms cause a reduction of charge inside the floating gate which is reflected in the output current. By sensing this change in the current value, one can determine the cumulative incident radiation on the device. A reference radiation dosimeter such as an ion chamber is used to determine the actual amount of ionizing radiation. The change in FG-MOSFET I $\mathrm{DS}_{\mathrm{D}}$ is then taken as a secondary measurement with respect to the reference device. This is the basic principle of FG-MOSFET devices as depicted in Figure 3.1. 


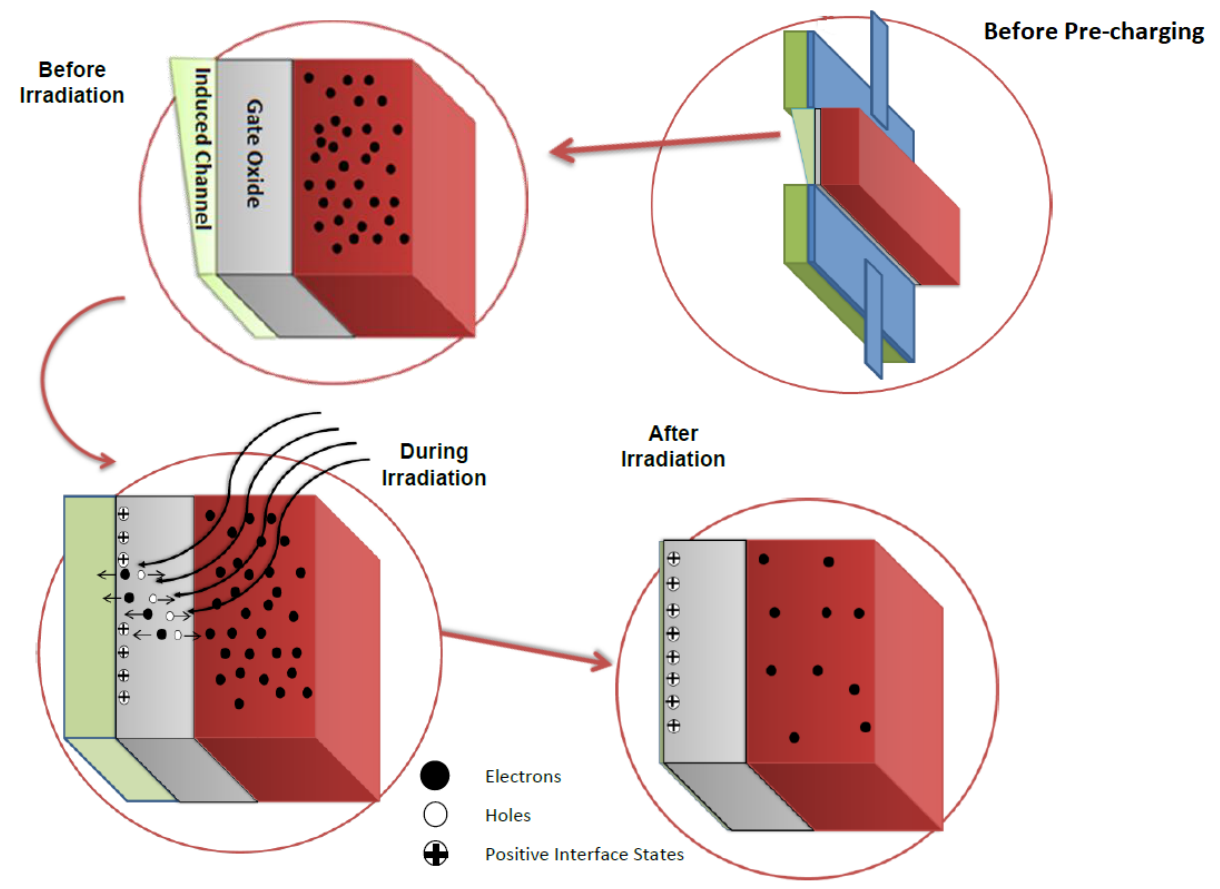

Figure 3.1: Simplified operation of an FG-MOS sensor [31]

\subsection{Design Variants and Preliminary Results}

To fulfill the sub-objective 3.1, it is proposed to design, fabricate, and test (electrical characterization and irradiation) variants of FG dosimeters as presented below and introduced in subsections 3.3.1-4:

1. Various FG-MOS devices, with(out) passivation

2. Various FG-MOS devices, with(out) metal protection

3. Various FG-MOS devices configured with a single or multiple fingers

To fulfill the sub-objective 1.3, it is proposed to experimentally explore the charging and discharging behavior.

Prior to presenting the irradiation exposure experiments in the following sections, 
it is necessary to explain the test setups, the devices used, and measurement techniques and calculations. These explanations are valid throughout the thesis for all the experiments unless otherwise mentioned.

\subsubsection{Radiation Test Setups and Techniques}

There are two X-ray sources that are used for the majority of experiments performed in this thesis:

1. An X-ray device with a commercial brand of "Faxitron ${ }^{\circledR} "$ shown in Figure 3.2;

2. An X-ray device with a commercial brand of "Raycell MK2 Blood Irradiator ${ }^{\circledR}$ " shown in Figure 3.4;

The first test setup involves the use of an X-ray source with the commercial name "Faxitron". This device is capable of producing X-rays in the approximate range of $5 \mathrm{keV}$ to $45 \mathrm{keV}$ by adjusting the voltage range from $0 \mathrm{kV}$ to $110 \mathrm{kV}$. This $\mathrm{X}$-ray range is usually noted as the diagnostic X-ray energy usually applicable to diagnostics and imaging fields. Figures 3.2 and 3.3 demonstrate such test setup.

The breadboard with the target sensor on it was placed at the center of the metallic tray at a distance of 10 inches directly under the opening of the X-ray lamp to establish a consistent test setup across all variants of the FG-MOS. Wires route out of the board to the measurement unit. A Keithley 2400 SMU was used to collect data samples and a Rigol LXI DG1022 was used to precharge the FG-MOS devices before each radiation session. More about the precharging stage is brought in the later sections.

Despite the fact that the Faxitron X-ray machine produces lower energy X-rays and 


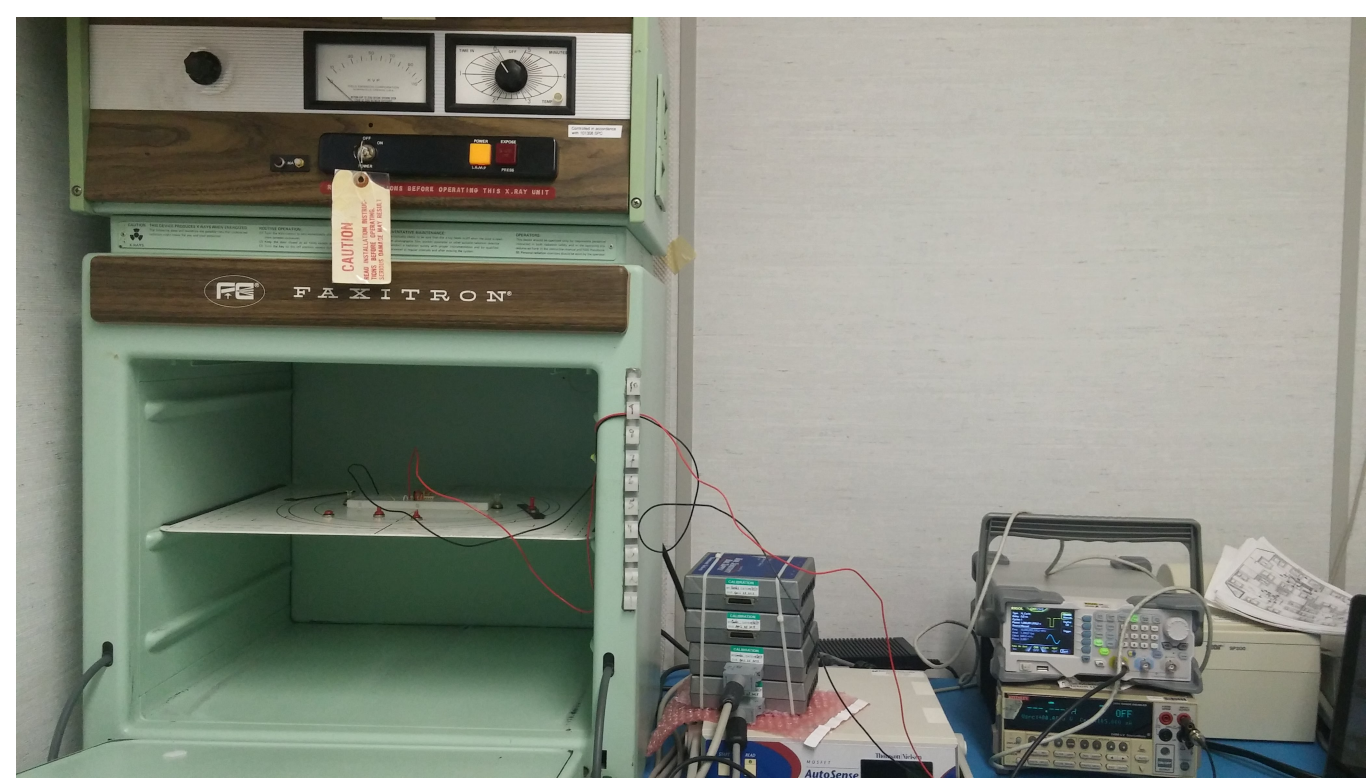

Figure 3.2: X-ray test setup

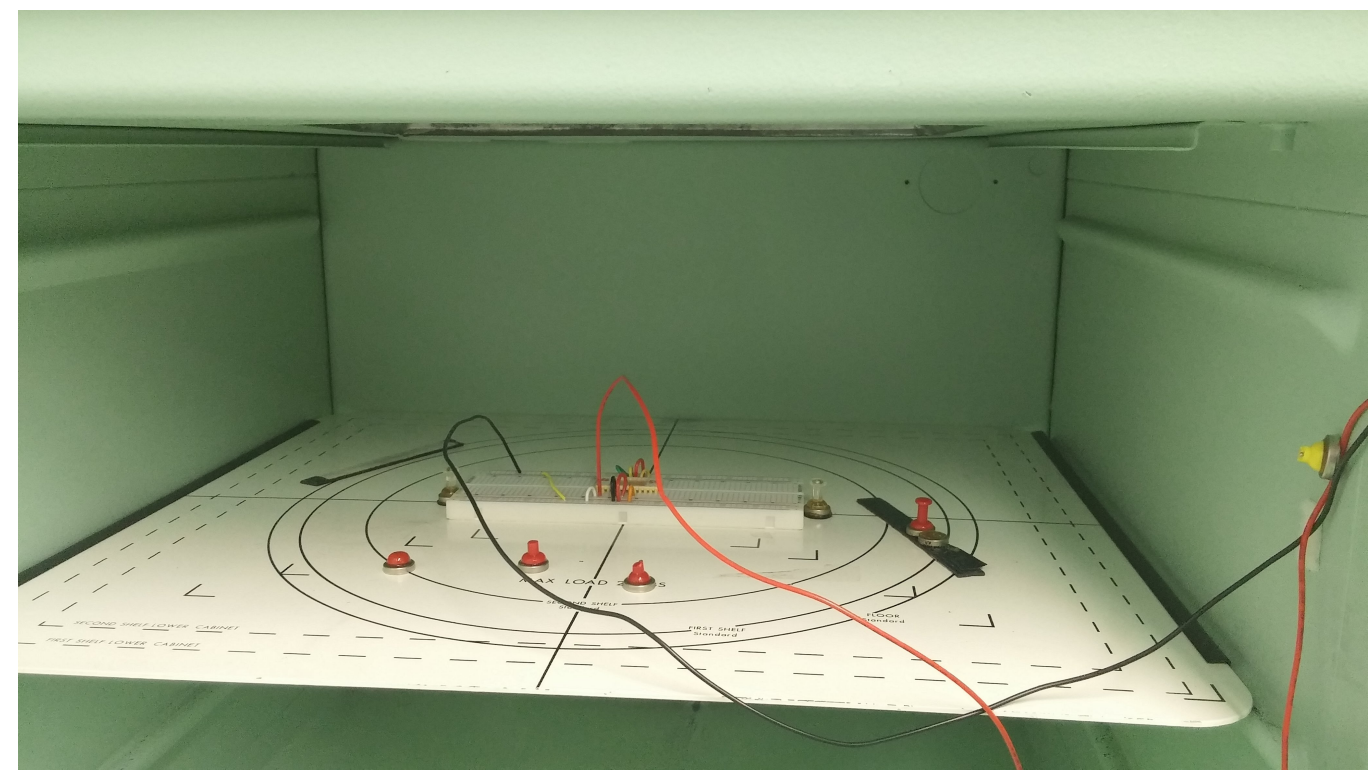

Figure 3.3: FG-MOS under radiation in the Faxitron X-ray machine 
lower photon flux than the Raycell MK2 $(2 \times 160 \mathrm{kVp}$ X-ray tubes each with $25 \mathrm{~mA})$, it is worthwhile to test the FG-MOS devices using this machine in order to establish whether they are sensitive to lower radiation energies or not. Throughout the testing, it was confirmed that any X-ray generated at peak voltages lower than $70 \mathrm{kV}$ would not result in significant sensitivity, hence radiation tests were carried out using $70 \mathrm{kVp}, 90 \mathrm{kVp}$, and $110 \mathrm{kVp}$ settings. However, only results of irradiation using X-rays produced at $110 \mathrm{kVp}$ will be presented throughout the chapter. The majority of the radiation tests using the Faxitron machine were done with the dial at $110 \mathrm{kVp}$ to acquire higher energy photons and possibly higher photon flux so to speed up the process of floating-gate discharge. This is due to the shortness of time available to perform the tests at the facility.

To determine the photon energy distribution in the X-ray machine, attempts were made to use an X-ray photon energy spectrometer with no success due to mechanical difficulties. There is no inlet devised on the outer body of the machine which would allow routing of a spectrometer or any absolute dose measurement reference such as an ion chamber. However, the setup described below presents a secondary approach to overcome this limitation.

To determine the dose rate of the machine at different peak voltages, production (reference) gate-connected MOSFET dosimeters of close dimensions to the devices presented in this section were used with a predetermined calibration factor. As per the documents generated internally by the providing company (Best Medical Canada Ltd.), a calibration factor of $14 \mathrm{mV} \mathrm{cGy}^{-1}$ can be applied to determine the dose to the dosimeters. Since the production (reference) dosimeters used are close in dimensions to the test subjects, it is safe to assume the same amount of radiation is incident on them. Since all the radiation testings are done against the duration of the exposure 
$(\approx 300 \mathrm{~s})$, it is essential to find the total dose per unit of time (dose rate) and the total cumulative dose.

Many sets of radiation tests were performed on the reference units for the duration of 300s to determine the absorbed dose. The reference MOSFETs are connected to a measurement box that records the change in voltage every second shown in second line of Eq.3.1. The dose rate and total dose calculations are shown below:

$$
\begin{aligned}
\text { Cal.Fac. } & =14 \frac{\mathrm{mV}}{\mathrm{cGy}} \\
\frac{\Delta V}{s} & =1.63 \frac{\mathrm{mV}}{\mathrm{s}} \\
\frac{1.63}{14} \frac{\frac{\mathrm{mV}}{\mathrm{s}}}{\frac{\mathrm{mV}}{c G y}} & =0.116 \frac{\mathrm{cGy}}{\mathrm{s}} \\
I_{D S-0}-I_{D S-1} & =\Delta I_{D S} \frac{\mu A}{\mathrm{~s}} \\
\text { Sensitivity } & =\frac{\Delta I_{D S} \frac{\mu A}{\mathrm{~s}}}{0.116 \frac{c G y}{\mathrm{~s}}}=\frac{\Delta I_{D S}}{0.116} \frac{\mu A}{c G y} \\
\text { TotalDose }_{300 s} & =0.116 \frac{c G y}{\mathrm{~s}} \times 300 s=34.8 c G y
\end{aligned}
$$

The second X-ray source used in the experiments in this thesis is equipped with two X-ray lamps operating at $160 \mathrm{kVp}$ placed vertically on top of and under the plastic canister (container) which in normal use contains blood bags. The device is shown in Figure 1.2. This device does not produce monochromatic X-ray photons, but rather a photon spectrum of $60 \mathrm{keV}$ to $80 \mathrm{keV}$ [81]. A calibrated "Radcal 9010 10x5-0.6cc" ion chamber was used to determine the dose rate of the machine as follows: 


$$
\begin{aligned}
\text { DoseRate } & =102.2 \frac{\mathrm{mGy}}{\mathrm{s}} \\
\text { CorrectionFactor } & =1.02 \\
\text { DoseRate }(\text { Corrected }) & =104.244 \frac{\mathrm{mGy}}{\mathrm{s}} \\
\text { DoseRate } & =6.254 \frac{\mathrm{Gy}}{\mathrm{min}} \\
\text { TotalDose }(\text { Sminutes }) & =6.254 * 5=31.273 G y
\end{aligned}
$$

The total dose calculated above is to the ion chamber, and experimentally determined total dose for the same duration of exposure to a canister filled with blood bags is 25 Gy [81].

It is important to highlight the fact that the blood irradiator unit's dose within the same 5 minute exposure duration is almost 90 times larger than that of the Faxitron unit. Also, the photon energies peak at $\approx 45 \mathrm{keV}$ for the Faxitron and $80 \mathrm{keV}$ for the blood irradiator. This difference helps explain the difference in results in the upcoming sections.

In this work, the actual dose to the MOSFET sensors is not taken as priority but rather the relative change in their characteristics within the duration of the irradiation session. MOSFET dosimeters always need to be calibrated against a reference measurement device such as an ion chamber before being exposed to radiation if they are to be used as a sensor.

Also it is important to bring to the attention of the reader that many publications report their FG-MOS radiation sensitivity in terms of the shift in the threshold voltage. In this work, the change in the channel current $\mathrm{I}_{\mathrm{DS}}$ is used to report the radiation 
effect. The voltage bias used throughout the experiments in Chapter 3 is kept consistently at $V_{\mathrm{DS}}=0.1 \mathrm{~V}$.

It is acknowledged by the author that the measurement of current change as a result of radiation exposure is dependent on the MOSFET width and length dimensions, whereas threshold voltage shift is a unique characteristic of a MOSFET regardless of the dimensions. With regards to the use of the shift in the threshold voltage, two significant points can be discussed: 1) No two fabrication technologies reported in the literature are identical in intrinsic characteristics such as $\mathrm{V}_{\mathrm{TH}}$ to make them absolutely comparable; 2) threshold voltage change as a result of irradiation is only applicable to gate-connected thick gate oxide older variants of MOS technologies in which permanent radiation damage in the gate oxide was responsible for this shift in the threshold.

As with the well-known electrically erasable programmable read-only memory (EEPROM) technology used for non-volatile memory (NVM) applications, the state ('0' or ' 1 ') of a charged or a discharged floating-gate MOS cell is determined by passing a current through the channel and converting that to a voltage through a sense amplifier stage.

Accordingly, in this chapter, the reported channel currents are used to draw conclusions which satisfy the objectives noted for this chapter. In future improvements to the results, a reference gate-connected identical MOS device can be used to indirectly determine the equivalent gate bias on the floating-gate device for the same drain-source bias.

In order to compare their dosimeter characteristics such as sensitivity, linearity, dosimetry information retention over time, noise immunity, and other possible measures, many publications utilize a known reference radiation source such as ${ }^{60}$ Cobalt. 
This synthetic radioactive isotope of Cobalt has a known half-life of 5.2747 years and emits two known gamma rays with energies of $1.17 \mathrm{MeV}$ and $1.33 \mathrm{MeV}$. Standard test setups such as the distance from the radioactive source, the area of exposure, the angle of exposure are considered for experiments. In this research work, we did not have access to the mentioned radioactive material; therefore, we cannot draw comparisons between the FG-MOS devices in this research and those of other publications.

The duration of exposure of the MOSFET sensors in the blood irradiator machine is set to 5 minutes in order to stay consistent in experiments done using the Faxitron Xray source. Figures 3.4 and 3.5 illustrate the test setup and equipments. Keithley 2400 SMUs and a Rigol LXI DG1022 pulse generator were used for the measurement of $I_{D S}$ and precharging respectively. The software application used to acquire measurement data is called "LabTracer 2.9 " provided by Tektronix ${ }^{\circledR}$.

Throughout the thesis, terminologies " $110 \mathrm{kVp}$ " and "blood irradiator" are used to distinguish between the X-ray machines and their respective characteristics.

With the test setups and measurement techniques described above, the following subsections describe the experimental results of the irradiation of the three variants of FG-PMOS sensors using each of the test setups.

\subsubsection{Floating-Gate Extension with/without Silicide Layer}

Silicide layer is automatically implanted on any poly-silicon layer on chip to reduce its resistance since the gate is used to route signal in short distances on chip. This compound layer is electrically conductive and reflective which could contribute to the degradation of radiation sensing of lower energy X-ray photons due to reflection and scattering. 


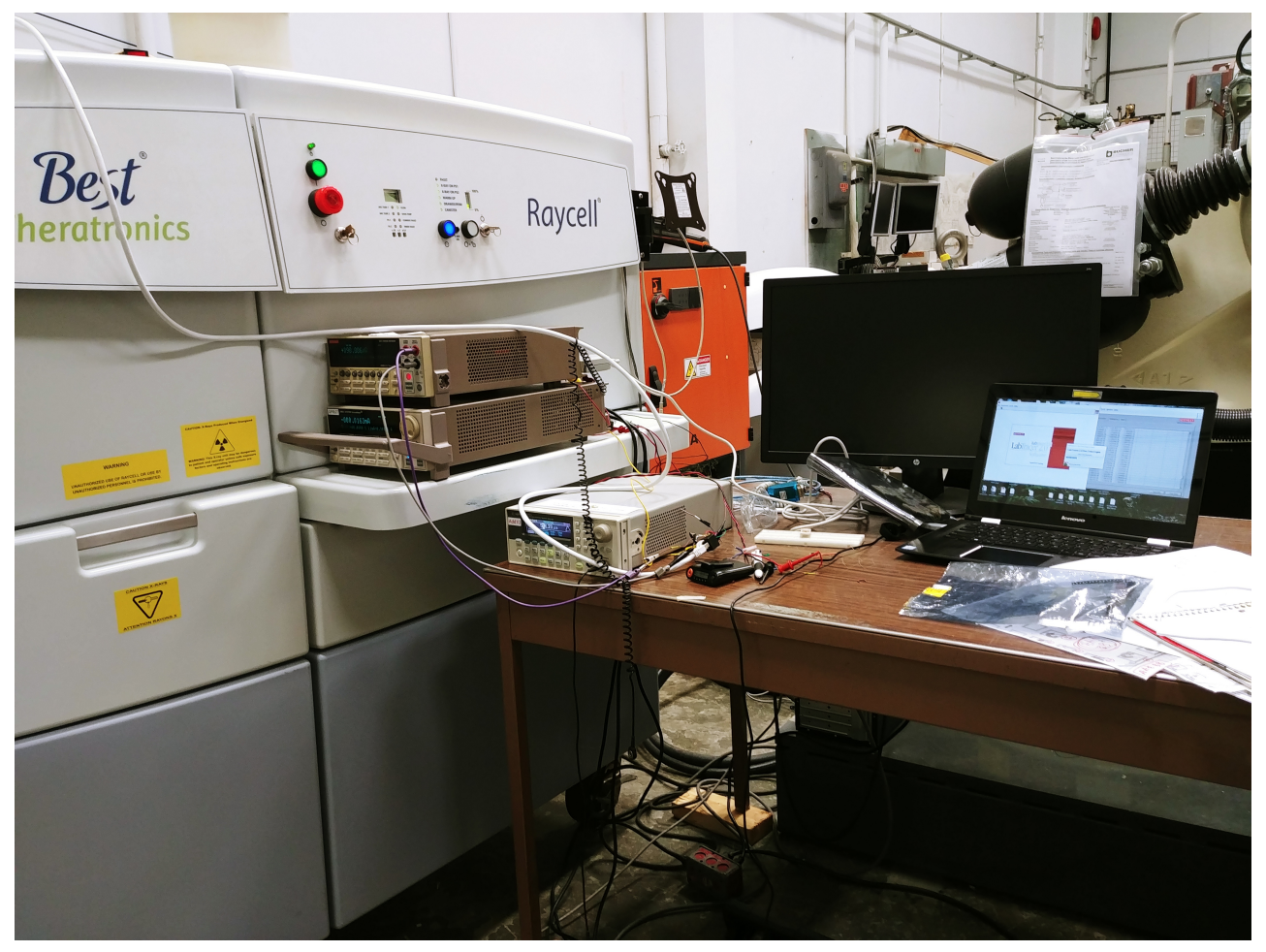

Figure 3.4: Blood irradiation machine test setup

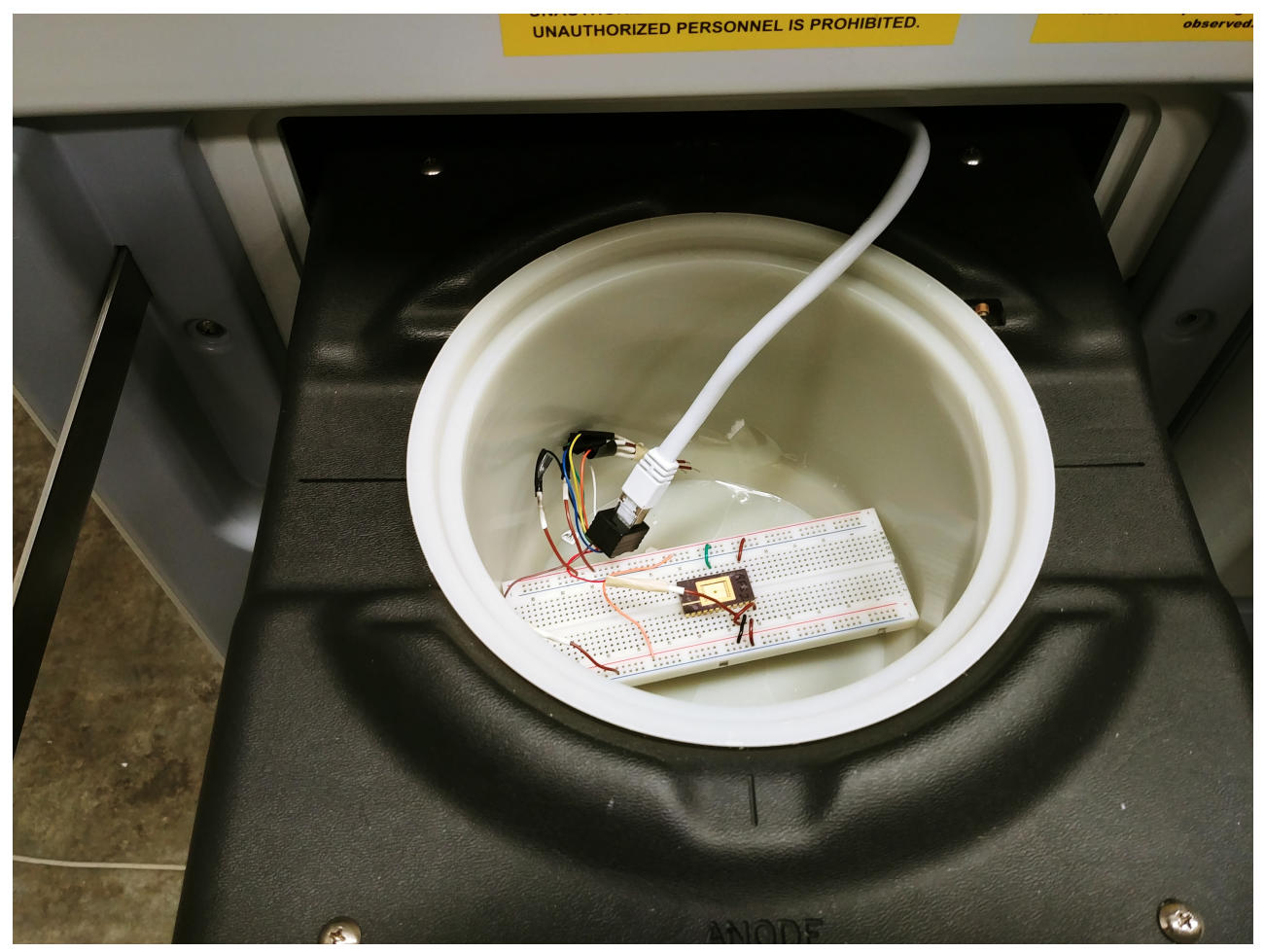

Figure 3.5: Blood irradiation machine test setup zoom 
This thesis explores radiation response comparison of an FG-MOS device having silicided poly-silicon gate extension and having the silicide deposition blocked. Figure 3.6 illustrates the layout of the two devices with identical dimensions of active and extension regions. Figure 3.7 depicts the same layout with all the physical dimensions and the contribution of the silicide layer's absence to the increase in sensitivity.

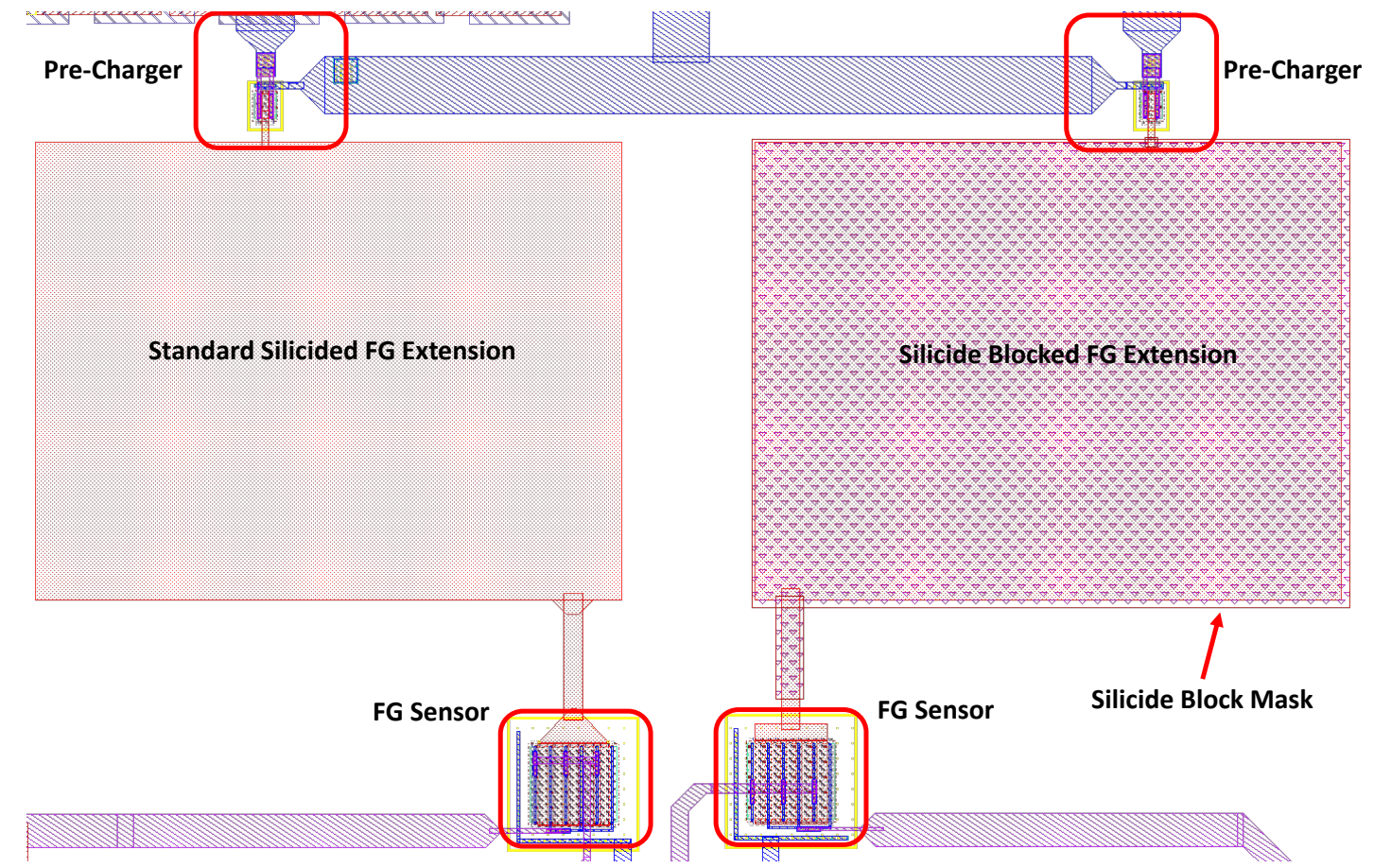

Figure 3.6: Silicided and non-silicided poly-silicon gate extensions

For clarity, Figure 3.8 shows the schematic of the silicide-blocked FG-MOS and its reference device.

To investigate the extent of radiation sensitivity of the FG-MOS devices, the pair have been irradiated under two different radiation sources.

Figure 3.9 illustrates the discharge of the floating gate under radiation at $110 \mathrm{kVp}$. A significant difference in sensitivity is observed between the silicide-blocked and the 


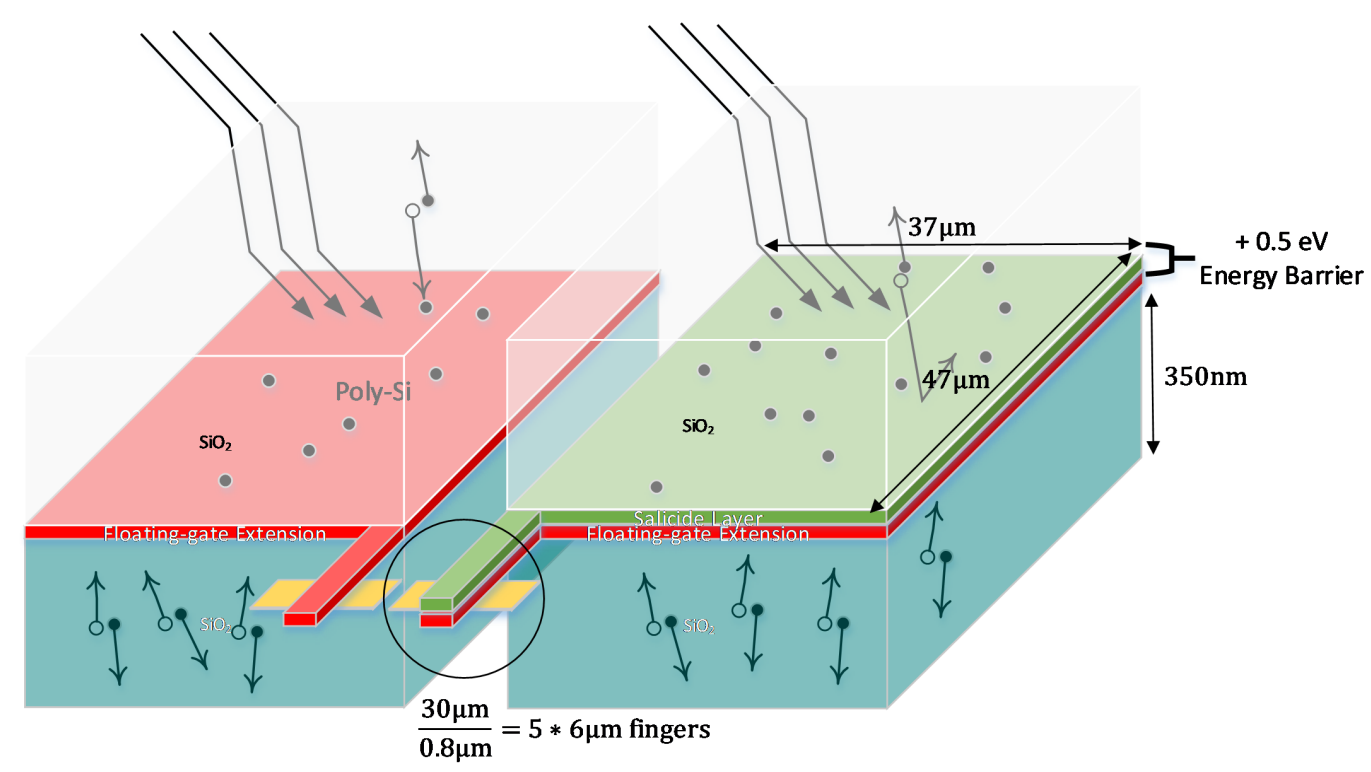

Figure 3.7: Depiction of silicided and non-silicided poly-silicon gate extensions
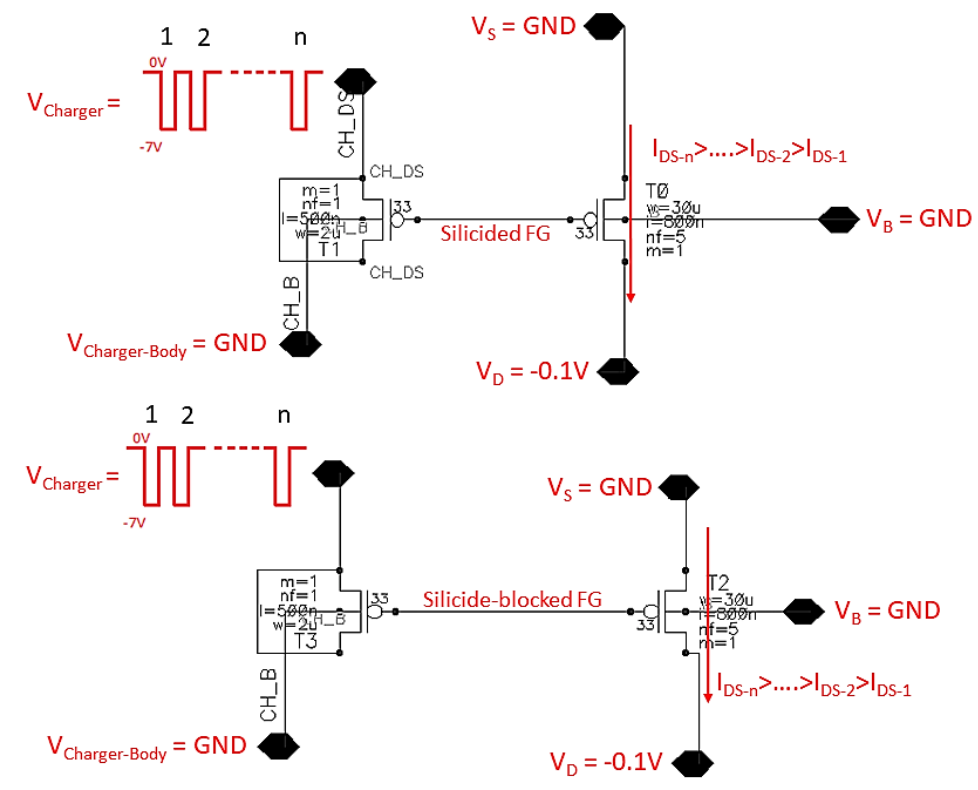

Figure 3.8: Schematic of the pair of FG-MOS devices 
silicided devices of otherwise identical properties. This difference is reflected through the difference in floating-gate discharge rates which in turn is reflected by a change in the channel current $\mathrm{I}_{\mathrm{DS}}$. Both the devices are biased at $-0.1 \mathrm{~V}$.

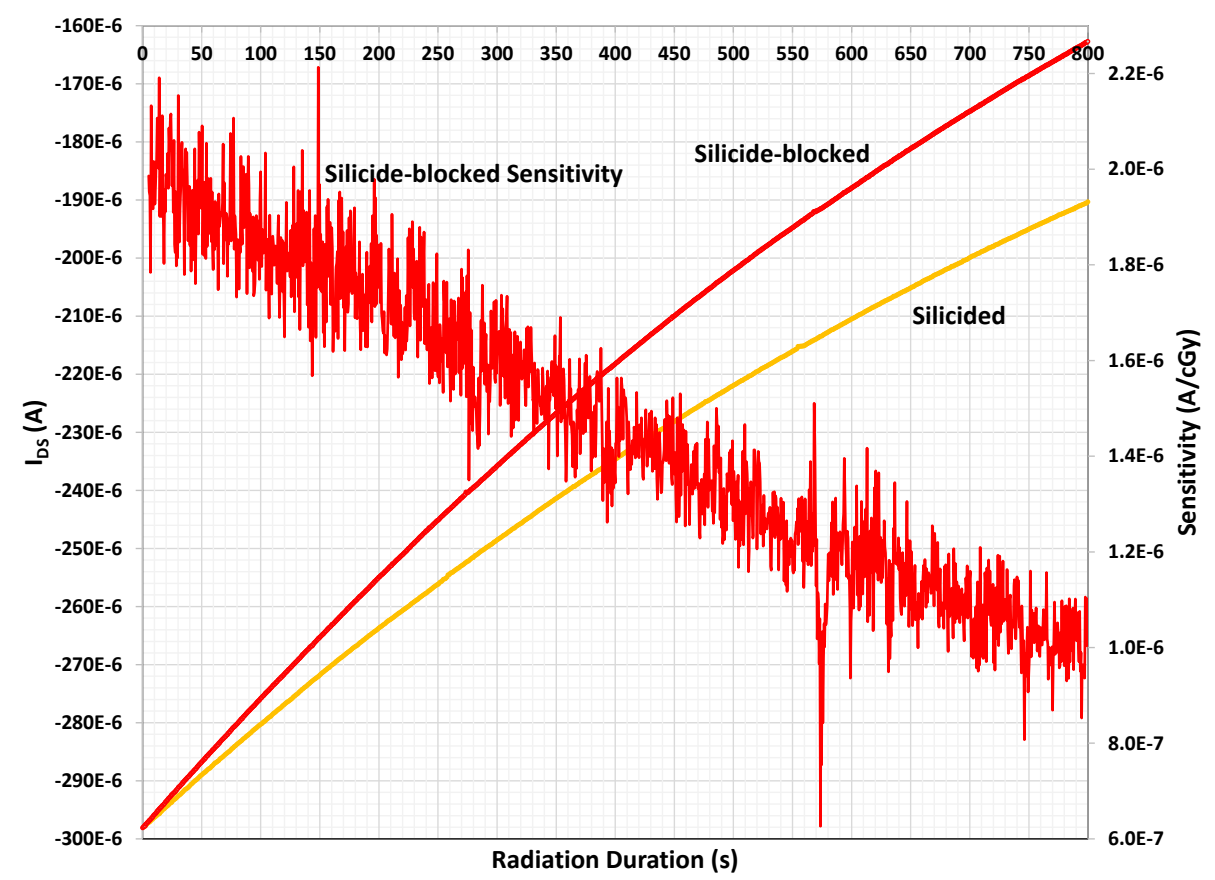

Figure 3.9: Radiation exposure of silicided and silicide-blocked devices to $110 \mathrm{kVp}$

This behavior is confirmed by the irradiation of other samples of the same configuration under the same setup. From Figure 3.9, it can be seen that the un-silicided version has a higher sensitivity. At the $800^{\text {th }}$ second, the silicide-blocked device shows approximately $140 \mu \mathrm{A}$ of reduction in channel current compared to $110 \mu \mathrm{A}$ for the silicided case.

Irradiation was performed for 12.5 minutes $(\approx 2.5 \times 5$ minutes $)$ in many repetitions in a number of devices. Under $110 \mathrm{kVp}$ X-ray, the silicide-blocked device showed $1 \%$ variation in response in repeated irradiations whereas the silicided variant showed $3 \%$ variation in irradiation response. The radiation sensitivity is also plotted on Figure 3.9 which shows a linear decrease from $\approx 2.1 \mu \mathrm{AcGy}^{-1}$ to $1.0 \mu \mathrm{AcGy}^{-1}$. One 
irradiation test was continued for 1730 seconds to find out whether the floating gate depletes completely under the above conditions, but it is observed that none of the FG-PMOS devices fully lost charge to completely stop the channel current.

Further analysis of the silicided and silicide-blocked devices point to the fact that the silicided sensors present a higher energy barrier for the secondary charged particles (electrons and holes resulting from incident X-ray photons) at the interface of $\mathrm{CoSi}_{2}$ and poly-Si which stops some of them crossing this interface and recombining with the excess electrons injected to the poly-Si floating gate. This results in enhanced sensitivity for the silicide-blocked device since more holes can reach the floating gate and recombine with the excess charge from the top. From $[82,83]$ this energy barrier can be calculated to be $0.47 \mathrm{eV}$ higher than without silicide.

In addition to the Faxitron X-ray irradiator, the devices are also tested in the Raycell ${ }^{\circledR}$ blood irradiator. These results are summarized in Figure 3.10. These results also show that the unsilicided devices have better sensitivity than the silicided ones with the former showing a channel current reduction of $190 \mu \mathrm{A}$ as compared to $160 \mu \mathrm{A}$ in the later ones. The pair of curves presented in Figure 3.10 is one from many results generated with the silicided and the silicide-blocked having $10 \%$ and $14 \%$ variation in results respectively at $600^{\text {th }}$ second. The curves of silicided and silicide-blocked devices follow each other which means that a $3 \%$ variation in one is followed by a $3 \%$ variation in the other. The sensitivity curve in Figure 3.10 shows a different behavior compared to the previous case which showed an almost linear decrease in sensitivity. An $85 \%$ decrease is observed versus $50 \%$ for the former case which is expected due to higher photon energy and flux in the blood irradiator than the Faxitron machine. Also is noteworthy the fact that as has been mentioned in many publications, MOSFETs' sensitivity is highly energy dependant. Also, as the electric field within the field oxide 
under the floating gate extension depreciates, charge separation efficiency decreases to a point where the MOSFET becomes insensitive to ionizing radiation. Any further electron-hole pairs generated will then be recombined due to the lack of a strong electric field.

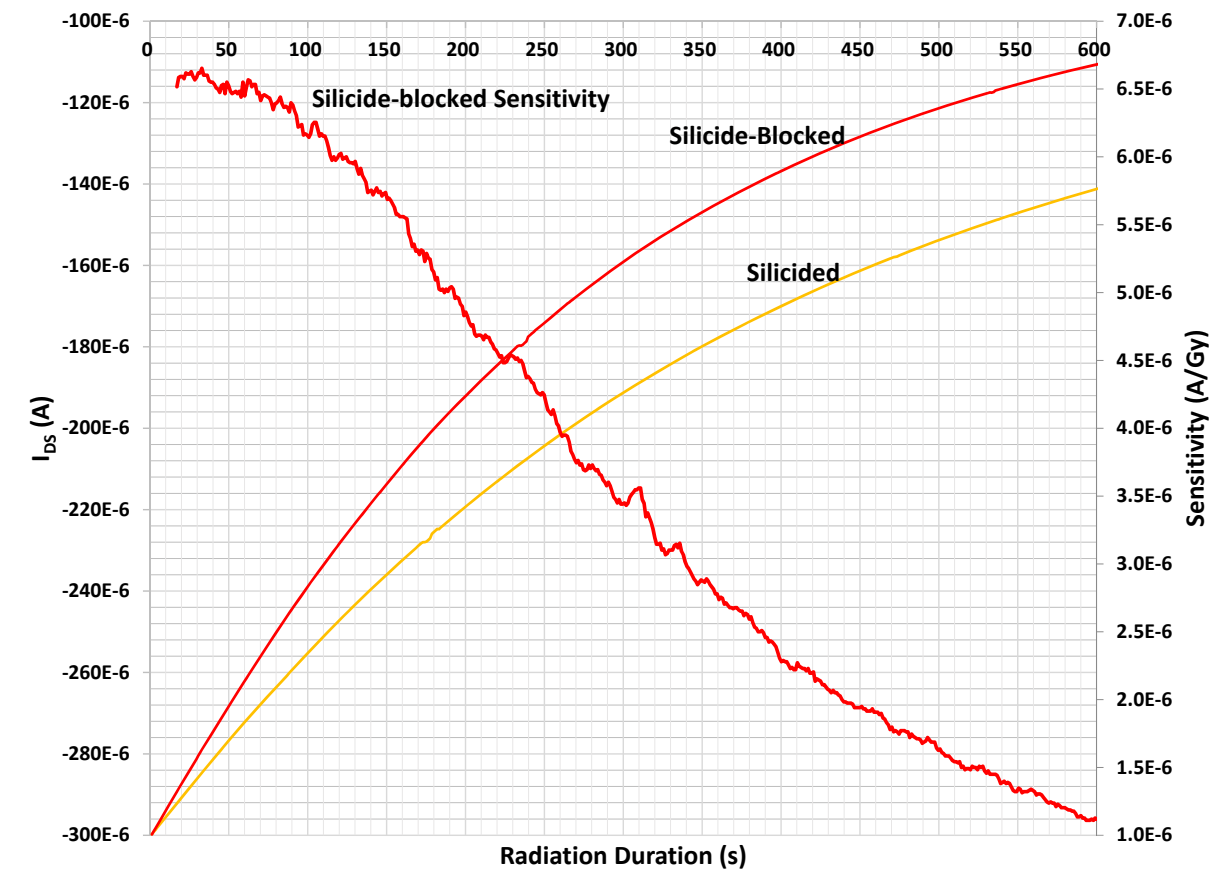

Figure 3.10: Radiation exposure of silicided vs. silicide-blocked device on a single chip using the Raycell Blood Irradiation Unit

The sensitivity of this FG-MOS variant decreases almost linearly within the first 300 seconds of the radiation session which is a desirable factor since this change in current can be converted to a linear voltage signal. 300 seconds is the time duration required to reliably irradiate a blood bag; therefore, a linear sensitivity response of the MOSFET is achieved for the useful period of the exposure. From 300 seconds to 600 seconds, sensitivity decreases sub-linearly which indicates diminished response. A lower FG-MOS radiation sensitivity leaves it more prone to noise-related implications when converted to voltage signals which makes this part of the response undesirable. 
The minimum resolvable dose detected depends on the noise within the MOS dosimeter; if higher signal-to-noise ratios are desired, larger MOSFETs need to be designed. Within the scope of this research, a great emphasis is placed on the difference in MOS variants' radiation responses while keeping all other variables out of the picture. Noise considerations and minimum detectable dose become important factors when a readout circuit is to be constructed to convert the current to a voltage signal. Such readout circuits are not explored in this Chapter.

With regards to pre- and post-irradiation stability of the FG-MOS devices, the same FG-MOS device was precharged and irradiated repeatedly. Before each irradiation session, the precharged FG-MOS was monitored for 5 minutes in order to ensure charge retention. Also, after each irradiation session, the FG-MOS device was monitored for 5 minutes in order to observe any possible current change such as a decrease, an increase, or fluctuations. At the end of the last irradiation session, the FG-MOS device was left connected for approximately 15 hours to ensure charge stability. As a result, no charge retention issues in pre- or post-irradiation were observed in any of the FG-MOS variants including the ones presented in the next sections.

\subsubsection{Floating-Gate Extension with/without Metal Protec- tion}

In works previously published [12,34], a grounded metal layer covering the floatinggate area protects it from nearby electric fields that could disturb the charge and also increases radiation sensitivity of the FG-MOS. Similar to the mentioned work, it is worthwhile to investigate this modification in this technology and for the specific $\mathrm{X}$-Ray energy range employed in this research work. 
For this study, a pair of floating-gate MOS sensors identical in dimensions and arrangement to the previous case were fabricated. One device was covered with the top metal layer ( $8^{\text {th }}$ metal layer) available in the $0.13 \mu \mathrm{m}$ RF-CMOS technology and the other side was fabricated without the metal layer. Figures 3.11 and 3.12 illustrate the fabricated devices' layout and the depiction of the two devices for more design clarity respectively.

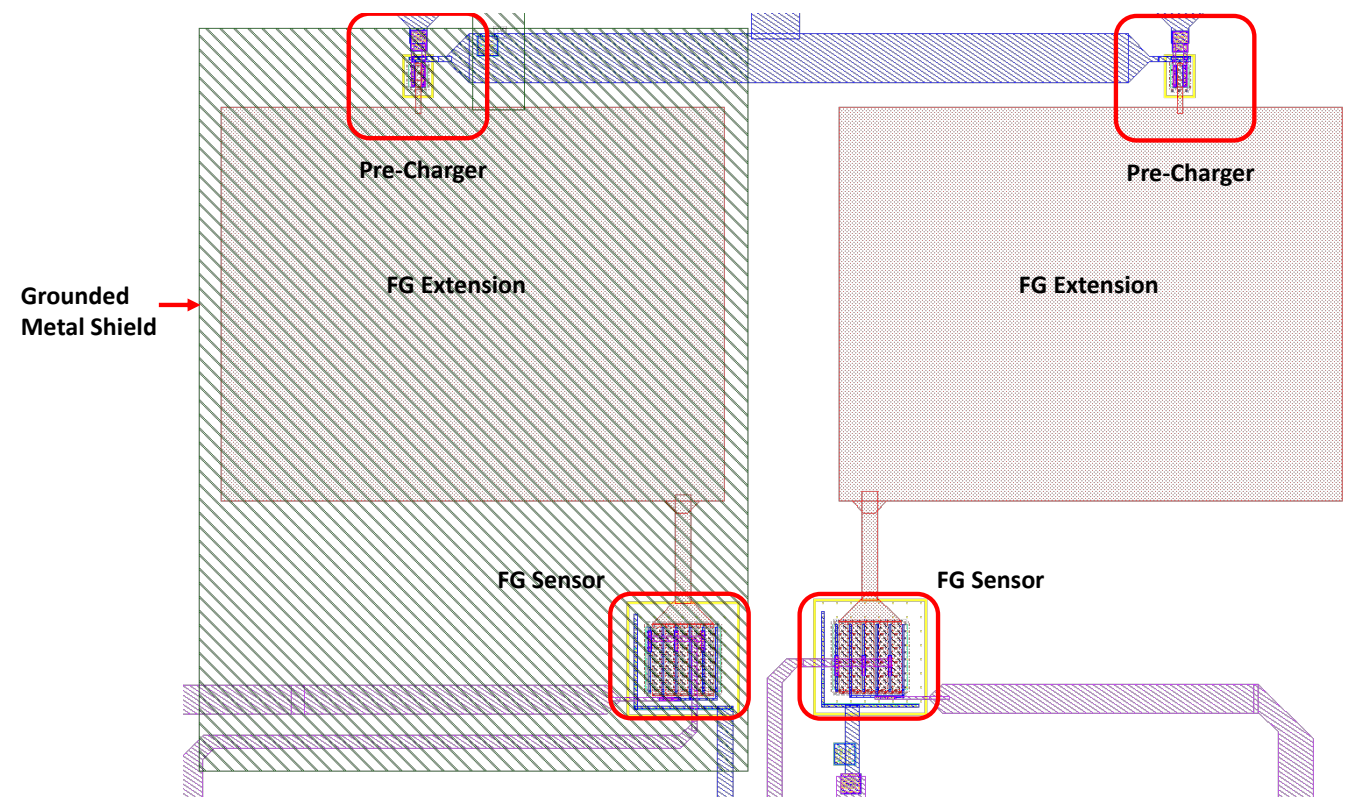

Figure 3.11: Grounded top metal layer covering the FG extension (left) and the exposed FG extension (right)

Radiation testing of two identical FG-MOS devices, one with grounded metal protection, and one without a grounded metal protection using the $110 \mathrm{kVp}$ source are presented in Figure 3.13.

It shows that the curves follow the same pattern with a $4 \%$ variation around the midpoint of $188.5 \mu \mathrm{A}$ at $600^{\text {th }}$ second for both variants. Other test results show that sensitivity variations cause the irradiation results to be indistinguishable between the 


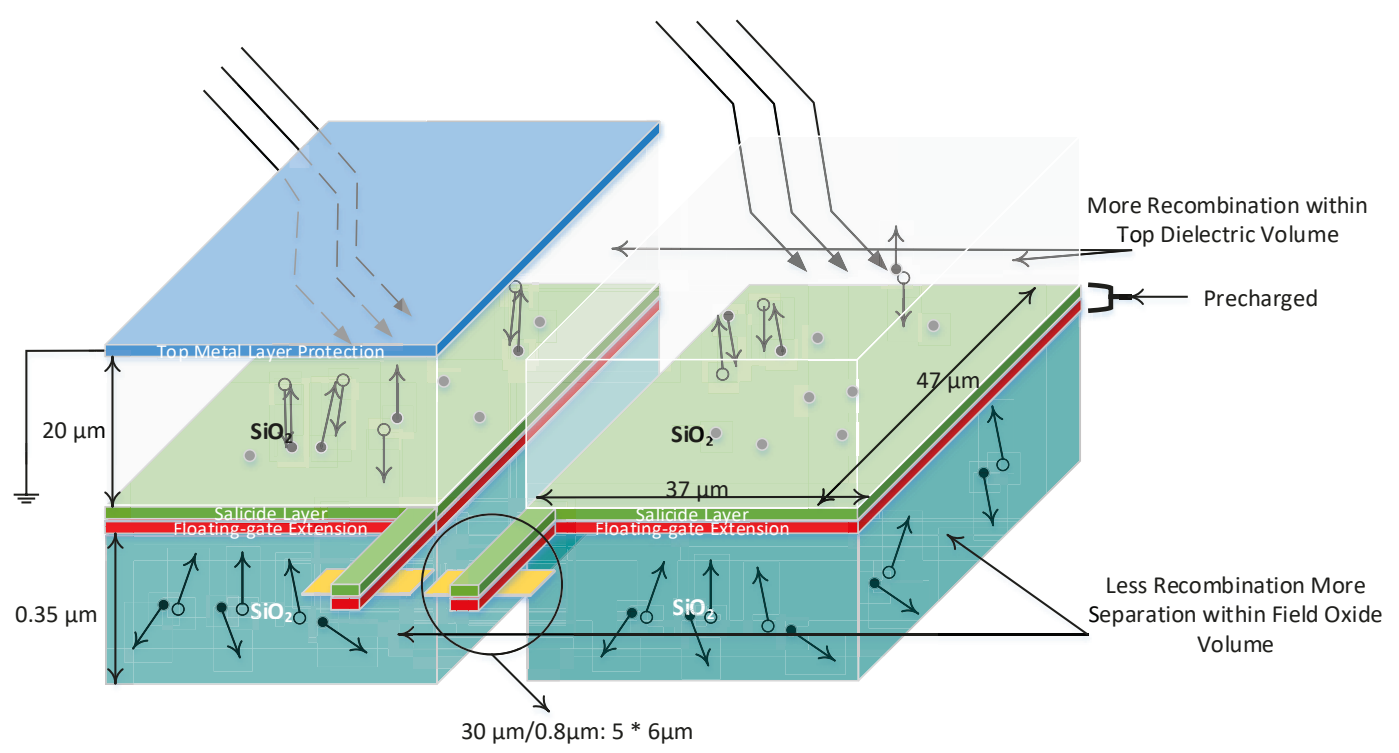

Figure 3.12: The depiction of the grounded top metal layer covering the FG extension (left) and the exposed FG extension (right)

sensors with and without metal coverage.

The presence of metal surfaces on top of the floating-gate structure does not have any significant effect on the device performance which is contrary to the conventional idea. Generally, it is well-known that having a metal shield would increase absorbed dose due to increased coupling between the floating gate and ground. The same might not be true in this case since that increase might be offset by the fact that there is a dielectric layer almost $20 \mu \mathrm{m}$ thick between the metal shield and the floating-gate surface. That may be the reason why any electron-hole pair generated within the dielectric region on top of the floating gate gets recombined before reaching the floating gate. Also the quality of the dielectric layer can be different from a technology to another which means there might be energy traps which would capture and recombine the generated charges. It would be beneficial to investigate whether there is an optimum metal layer distance from the floating-gate surface that contributes to the 


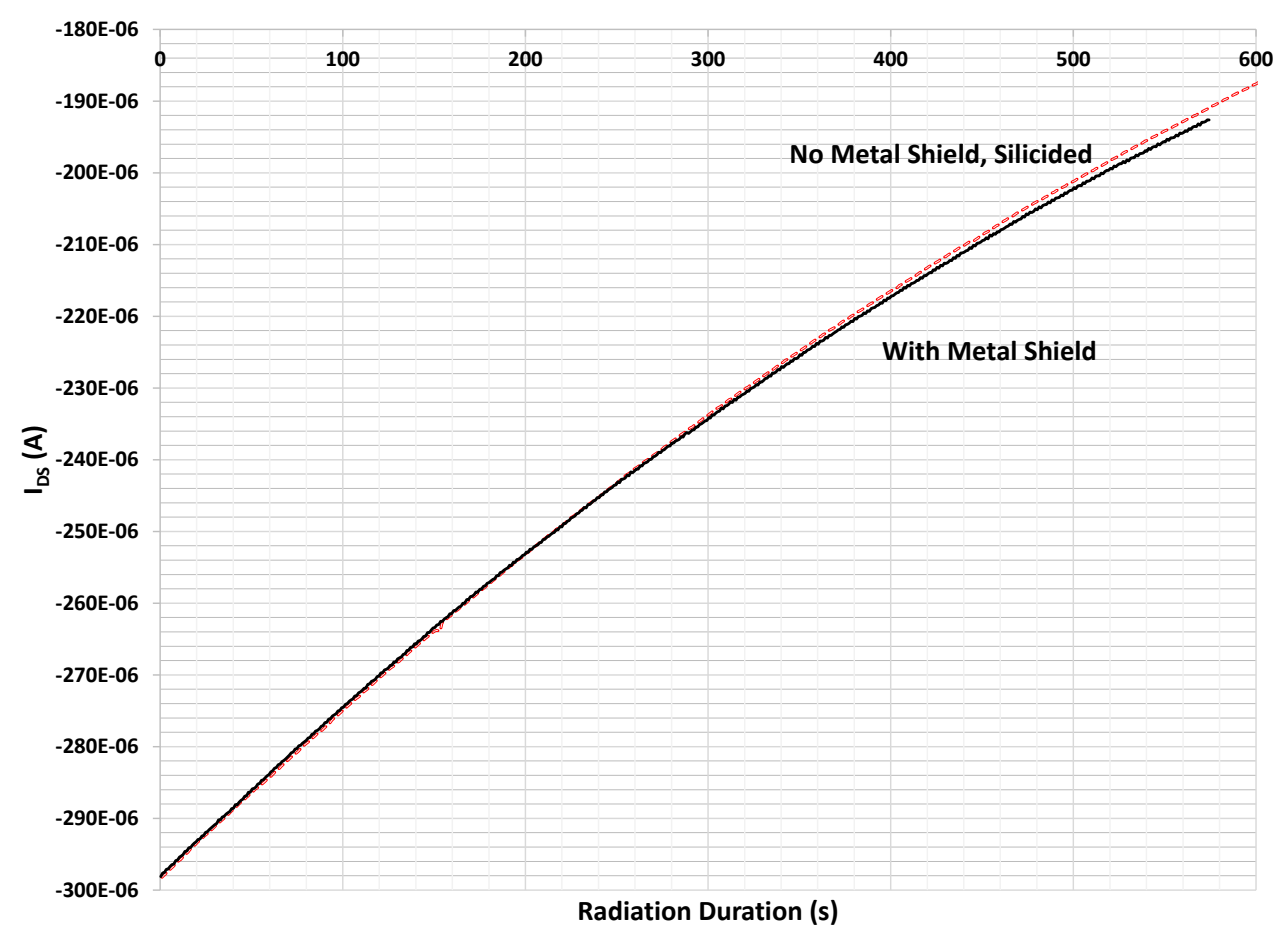

Figure 3.13: Metal shielded and no metal shield covered FG sensor exposed to $110 \mathrm{kVp}$ X-ray

sensitivity of the sensor. In this case, the highest metal layer was chosen, but in the future, other metal layers closer to the sensor could be investigated.

The blood irradiator is used next to investigate the behavior of the devices under radiation. The results are shown in Figure 3.14.

Despite the fact that the 2 curves show a difference in behavior, further irradiations of multiple devices have shown a variation in results of $\approx 14.5 \%$ at $300^{\text {th }}$ second without a correlation which means that there is no clear distinction between results of metal shielded and not metal shielded devices. This agrees with the results of the 110 kVp X-ray source. 


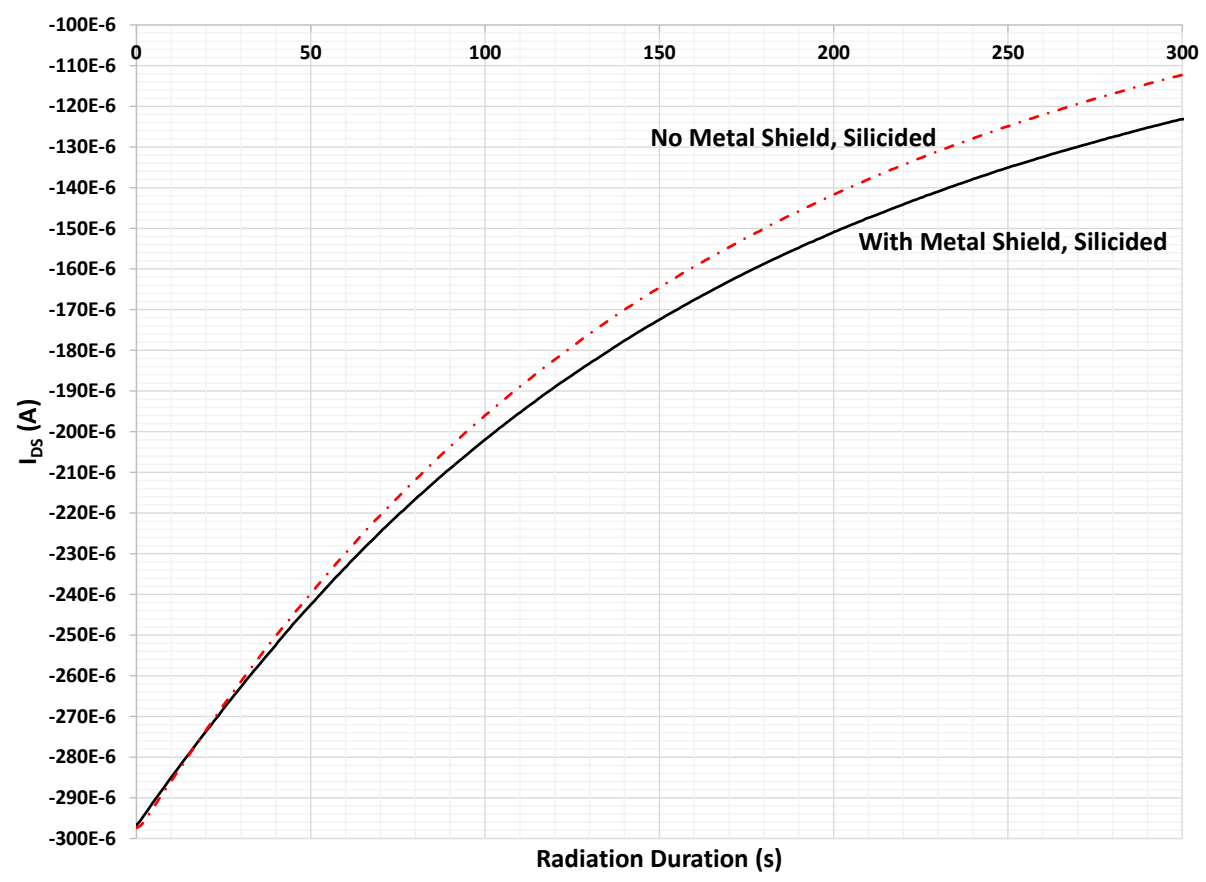

Figure 3.14: Metal shielded and no metal shield covered FG sensor exposed to X-ray from blood irradiator machine

\subsubsection{Floating-Gate MOSFET with/without Multiple Fin- gers}

MOS transistors could be laid out having only one finger or multiple fingers. Some of the benefits of having multi-fingers is reduction in chip space occupied by the transistor and more immunity against fabrication process variations which could lead to characteristic $\left(V_{t h}, L_{\text {eff }}\right.$ dimension) variations. On the other hand, multi-finger transistors can have additional parasitic capacitance which could affect the sensitivity. Exploring this option is important since the working principles of an FG-MOS dosimeter depends on a combination of MOS capacitances as illustrated in Figure 3.15 and described by Eq.3.3.

$$
\Delta I_{D}=\frac{g m_{F G}}{C_{f s}+C_{f d}+C_{f b}+C_{f o x}+C_{f b p s g}+C_{o x}} \times \frac{D}{W_{e-h}} \sum_{i} f_{i} \rho_{i} A_{i} t_{i}
$$


where, $D$ is the total dose (energy deposited per unit mass), $W_{e-h} \approx 18 \mathrm{eV}$, the electron-hole pair generation energy, $g m_{F G}$, the trans-conductance of the transistor, $f_{i}$, the fraction of electron-holes not recombined, $\rho_{i}$, the material density $\left(\mathrm{SiO}_{2}\right), A_{i}$, the area of capacitor, and $t_{i}$, the thickness of the dielectric [16].

As can be seen, there are a variety of capacitances that eventually constitute the overall effect on the electrical performance of a MOSFET. These include the oxide capacitances $C_{O X}, C_{f B P S G}$, and $C_{f O X}$, the depletion capacitance $C_{d e p}$, while there are additional capacitances between the FG and the device terminals $C_{f s}, C_{f g}, C_{f d}$. $Q_{f g}$ is the total charge that is accumulated on the gate terminal.

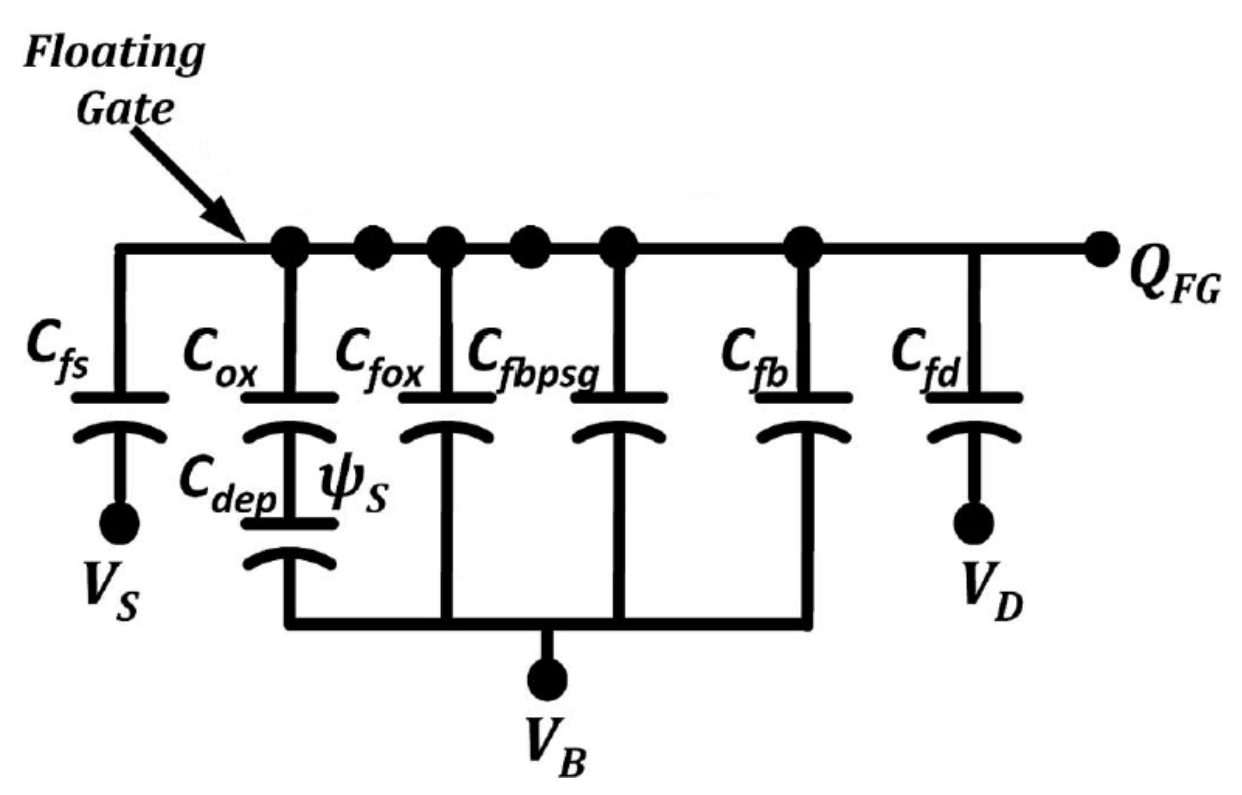

Figure 3.15: Capacitor model for the FG-MOS device [16]

Bearing the above schematic in mind, Figure 3.16 illustrates the layout view of the multi-finger against single FG-MOS device. The two devices have identical $\frac{W}{L}$ values. Moreover, this layout was done on a separate batch (same technology, different run) from the ones shown in Figures 3.6 and 3.11 which helps investigate, as an additional measure, radiation sensitivity variations across different chips and runs, even though 
it is nominally assumed to be the same. All the FG-MOS devices fabricated so far have $\frac{W}{L}=\frac{30 \mu \mathrm{m}}{0.8 \mu \mathrm{m}}$ and gate extension of $47 \mu \mathrm{m} \times 37 \mu \mathrm{m}$ which sets the ratio of gate extension to active region to $\approx 75 \%$. Antenna rules dictate a maximum ratio of $100 \%$ in order to reduce the chance of gate oxide damage during the fabrication steps. $75 \%$ ratio seems reasonably safe to design considering the fact that floating gates do not have a means to discharge the excess charge accumulated on them during fabrication.

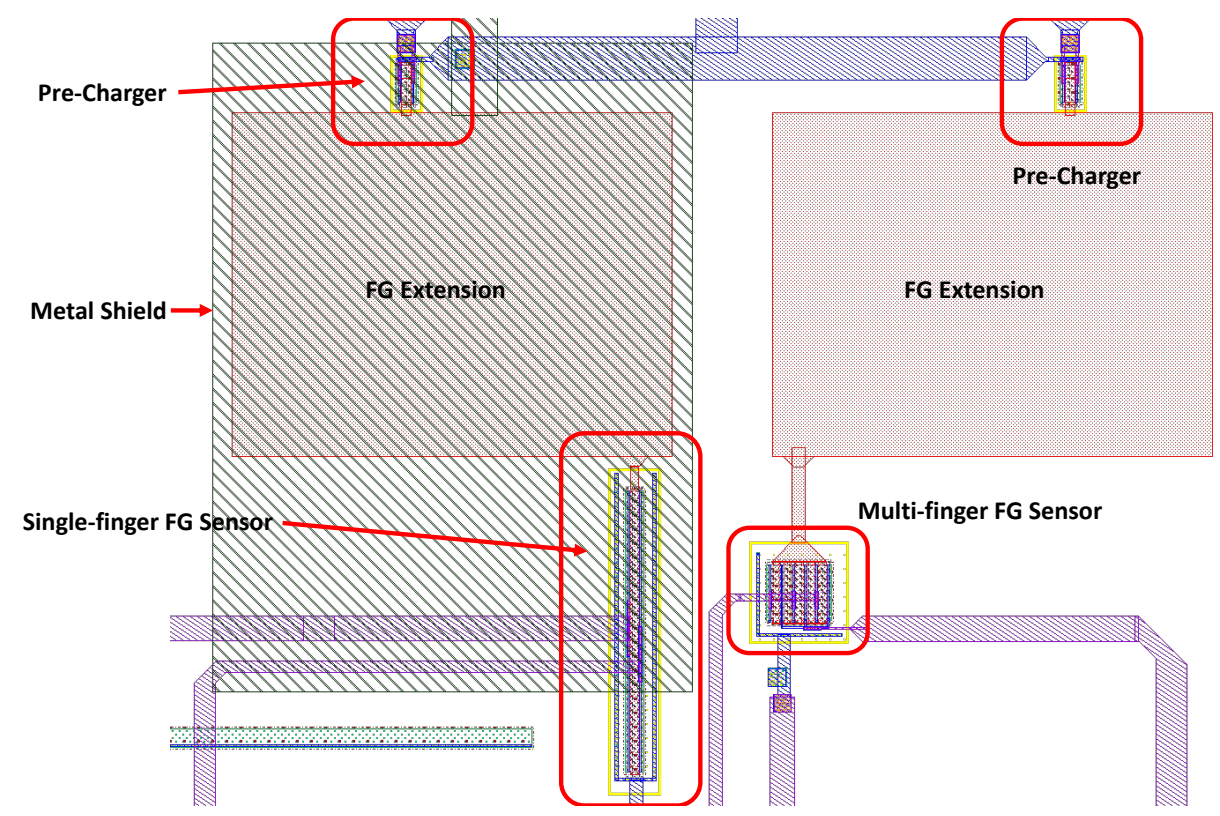

Figure 3.16: Single transistor (left) vs. multi-finger transistor (right) FG-MOS

Now keeping this in view, an analysis is provided in Figure 3.17 for the effect of using multi-finger gate as opposed to single finger devices. The variation in the channel current for the two configurations is small for the same intensity of radiation. This slight difference is due to the additional gate capacitance of the multi-finger devices. This capacitance comes due to the presence of multiple fingers in the gate implementation which introduces mutual capacitance among them. This is something well known to the circuit designers. Due to this change in the gate capacitance the sensitivity of multi-finger MOSFETs is better than the single finger counterpart. 


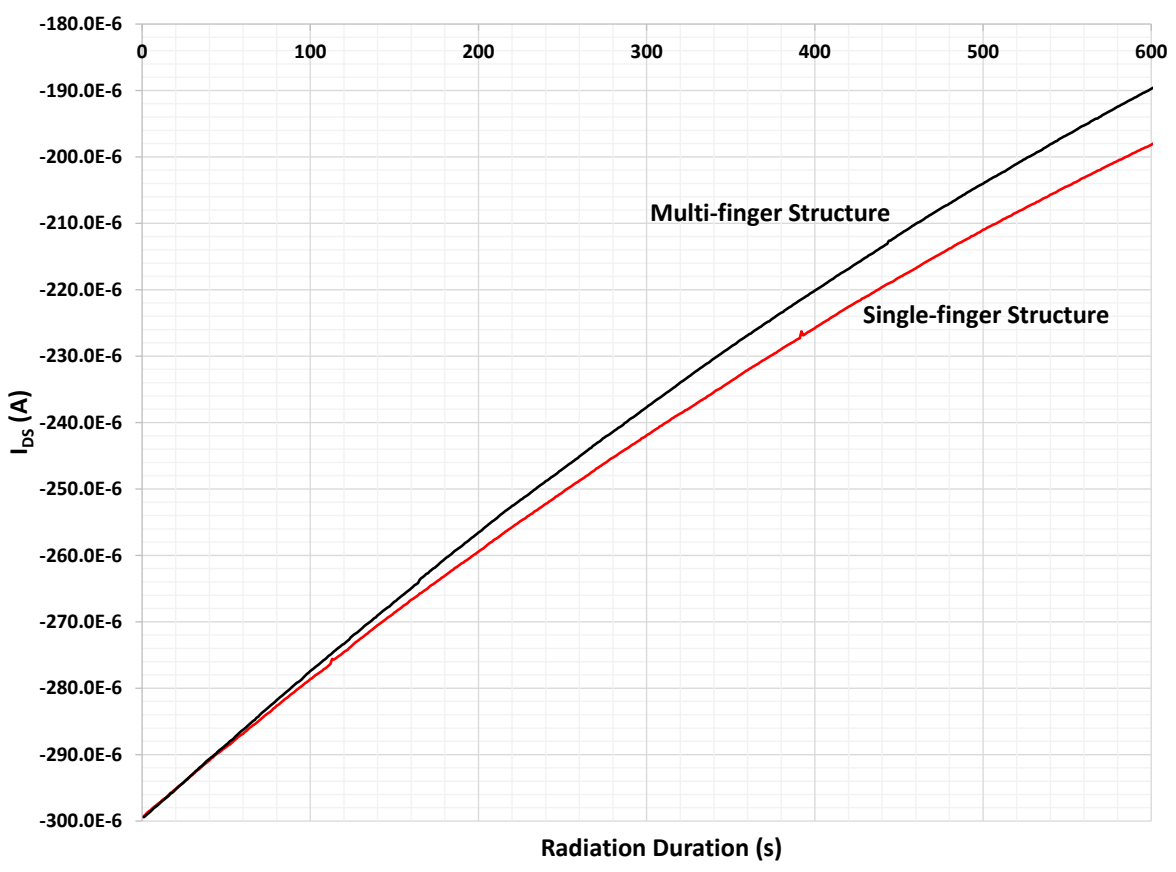

Figure 3.17: Single-finger and multiple-fingers structures exposed to $110 \mathrm{kVp}$ X-ray

Specially at higher dose levels this difference will become more apparent and can be exploited in the sensor design. The radiation results' variation is $\approx 2 \%$ and there is a distinguishable difference in results in the devices tested.

Figure 3.18 illustrates a sample radiation exposure measurement of the multi-finger and single finger device to the X-ray from the blood irradiator machine. The multifinger device shows a higher sensitivity compared to the the single finger one which is in agreement with the $110 \mathrm{kVp}$ X-ray source results. The variation is results for this design is $\approx 5 \%$. 


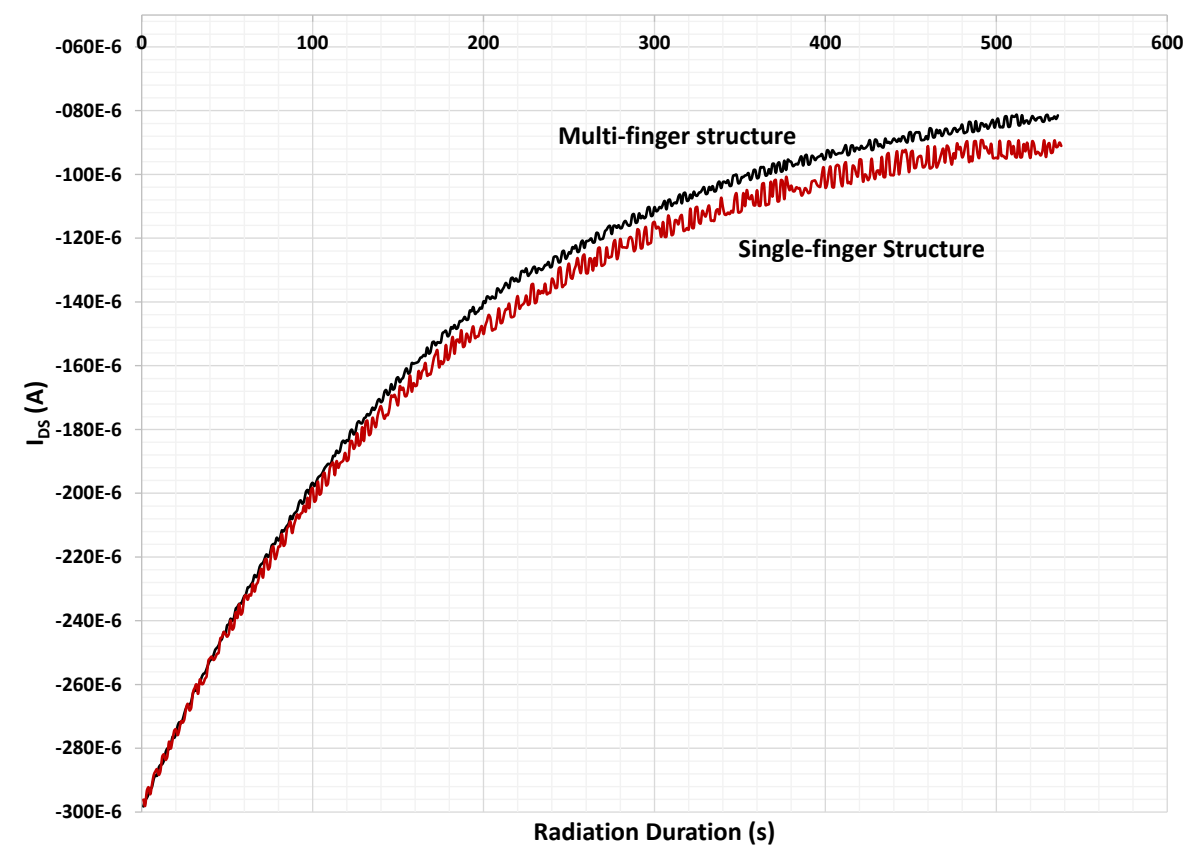

Figure 3.18: Single-finger and multiple-fingers structures exposed to X-ray from the blood irradiator machine

\subsection{FG-MOS Charge/Discharge Cycles}

Numerous charging and discharging tests have been performed on the floating-gate devices using pulse generators in order to determine the pulse height and widths necessary. These tests are important for FG MOSFET devices because of their use in the intended application of radiation dosimetry. It is important to minimize the complexity of the circuitry which will be implemented to carry out the automated charging/discharging task. Knowing that a potential structure such as a positive/negative pulse generator is required on chip, it is pivotal to study the charge/discharge characteristics of the FG-MOS devices. Common practice in charging the floating-gate MOSFETs in applications such as non-volatile memory devices is the hot carrier injection (HCI). A large bias across the drain/source while applying a large voltage on a control gate above the floating gate is maintained that causes a large current $\mathrm{I}_{\mathrm{DS}}$ in the device. This large current creates electron-hole pairs in the channel. A percentage 
of these generated charges are accelerated through the gate oxide and placed onto the floating gate, hence charging the device. As stated above, this practice requires a large current through the channel, but in the case of a low power sensor with limited power budget, it is difficult to produce. It was determined that applying pulses to the shorted PMOS precharger device illustrated in many of the figures including Figure 3.8 could charge and discharge the floating gate without the use of a large current through the channel.

The pulses used to charge/discharge the floating gate have certain characteristics: 1) polarity, 2) pulse width, 3) pulse height, 4) duty cycle, and 5) the number of pulses, which will be discussed here.

The discharge process involves applying positive polarity pulses to the shorted PMOS and its nWell body while the substrate is grounded. This causes the nWell body to the p-doped substrate diode to be reverse biased during the positive pulse causing the depletion of excess electrons from the floating gate, hence reducing the FG-MOS device's channel current $\mathrm{I}_{\mathrm{DS}}$. The tunneling effect utilized in this process depends on the gate oxide thickness through the formula below: [84]

$$
T(E)=\frac{1}{\sqrt{\frac{2 m}{h^{2}}\left(V_{0}-E\right)\left(x_{2}-x_{1}\right)}}
$$

where $T(E)$ is the transmission coefficient of electron, $m$ is the mass of electron, $h$ is the modified Planck's constant, $V_{0}$ is the potential in the barrier, $E$ is the energy level of the electron outside the barrier which is less than $V_{0}$ and $\left(x_{2}-x_{1}\right)$ is the thickness of the barrier which is the gate oxide thickness.

Voltage pulses lower than a certain value would not cause tunneling. Through experiments, $5.5 \mathrm{~V}$ was found to be the limit below which no discharge would occur. 
For this experiment, a random FG-MOS device was chosen, which was precharged to close to the maximum possible charge on the floating gate which corresponds to the maximum amount of $I_{D S}$ at a set drain/source bias of $-0.1 \mathrm{~V}$.

As mentioned above, pulse heights below $5.5 \mathrm{~V}$ do not discharge the FG-MOS. Hence, as seen in Figure 3.19, pulse height values below $5.5 \mathrm{~V}$ were omitted. Pulse heights of $6.0 \mathrm{~V}$ or $6.5 \mathrm{~V}$ do discharge the $\mathrm{FG}$, but not completely, even though longer pulse widths were applied meaning that no matter how many pulses of different pulse widths are applied, beyond a certain point, no discharge occurs. Hence, naturally, pulse height was increased to $7.0 \mathrm{~V}$. This value discharges the FG effectively. It is capable of completely discharging the FG.

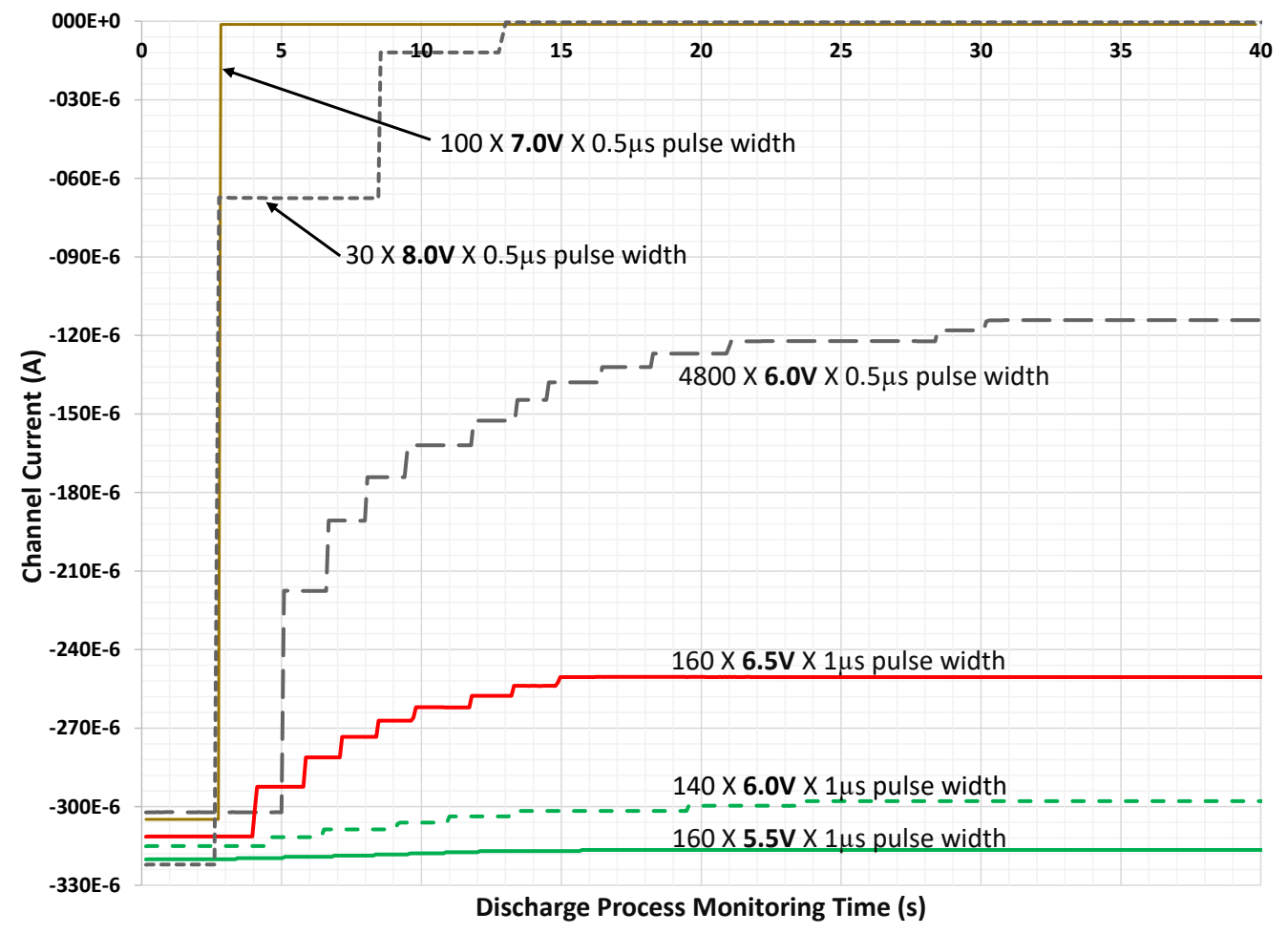

Figure 3.19: Discharging process of an FG MOSFET by applying different pulse heights and numbers. The values in bold signify the different amplitudes of pulses applied during the discharge process. 
Experimentally, it was determined that an optimum pulse train of 100 pulses each of $0.5 \mu \mathrm{s}$ (50\% duty cycle) depletes the floating gate. This is the minimum requirement to completely discharge the floating gate. Voltages higher than $7.0 \mathrm{~V}$ could surely discharge the FG, but also require higher pulses generated which in turn means more complexity, more stages of voltage multipliers and more power consumption.

Having the optimum pulse height for discharging the FG, it would be ideal to be able to charge the FG by the same pulse height value, but in the opposite polarity $-7.0 \mathrm{~V}$. This value is desirable since once a positive pulse generator is designed, all one would need is to convert it to the negative value by adding one extra stage.

Without investigating whether any other negative pulse height values would be effective, attention was focused on determining the optimum pulse width and duty cycle in order to minimize the time to charge the FG. To satisfy this point, many pulse trains of $-7.0 \mathrm{~V}$ with different pulse widths and periods and pulse counts were tested. The negative pulses were applied to the shorted drain/source of the precharger PMOS device. In this configuration, the body of the PMOS (nWell) could be left floating or tied to the p-substrate (GND or common). This configuration would stop any junction diode within the structure to forward bias during the precharging stage. To make the experiment efficient, the pulse width was chosen to stay at $0.5 \mu$ s as was in the case of discharge setup and only duty cycle (pulse period) and pulse counts were experimented with. Another important point to make here is that all the different variants of FG-MOS devices have the exact same precharger/discharger structure hence, the charge/discharge cycles were evaluated repeatedly on one device only. This also helps provide another conclusion as to whether or not the oxide breaks down under the pulses applied at the mentioned voltages.

Two separate groups of experiments were performed. Initially, manual single pulses 
were applied to the precharger device (shorted PMOS). By single application of each pulse to the FG, the behavior of the channel current was monitored in order to observe any rebound or fluctuation right after the pulse application. Figure 3.20 shows the experimental results. It can be seen from the figure that except for the yellow trace all other ones indicate precharging to almost the same value of channel current. The yellow trace simply shows lower number of pulses applied to it, which in turn shows lower current.

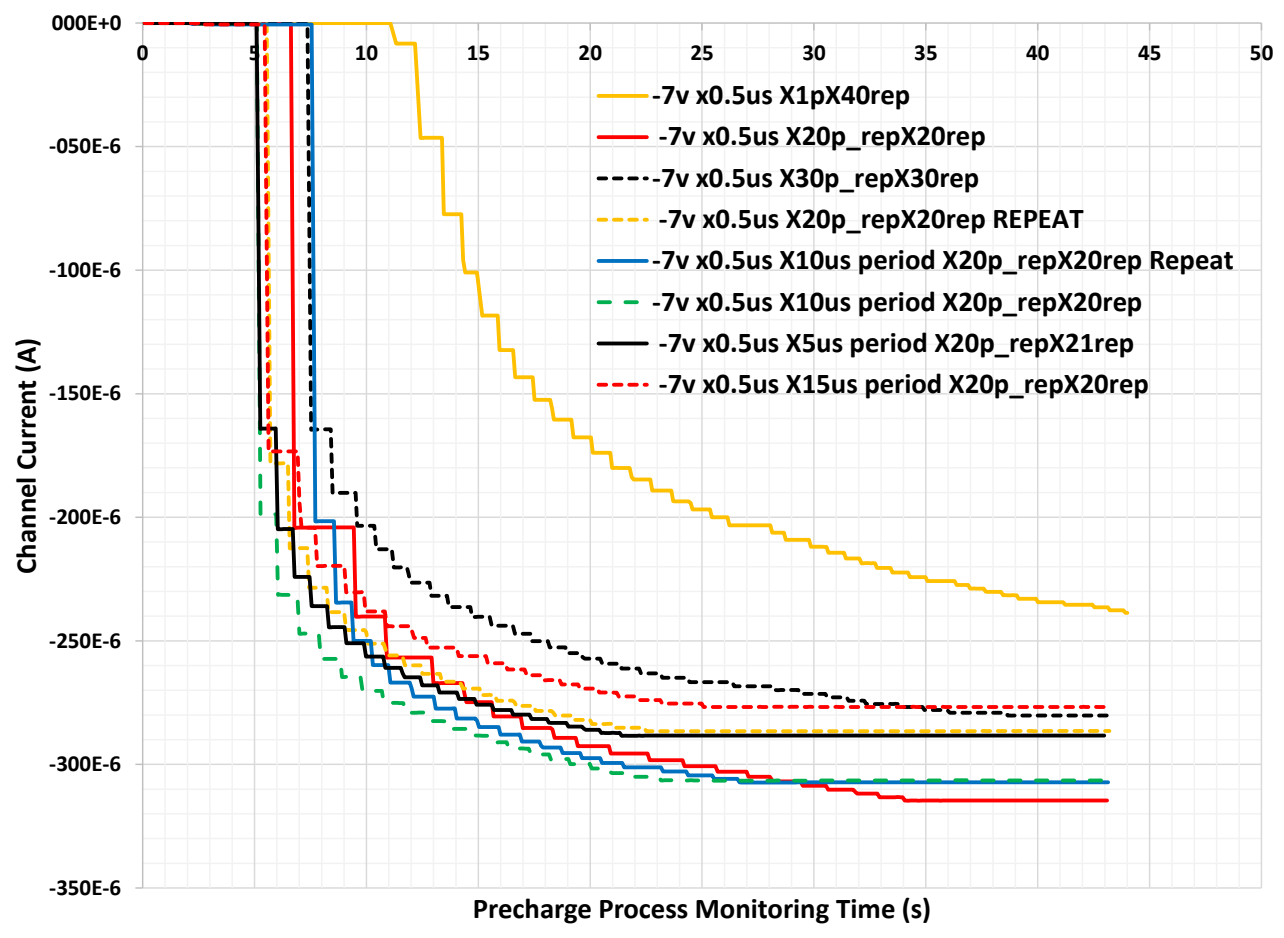

Figure 3.20: Manual precharging

Automated pulses were applied as shown in Figure 3.21. Pulse information is shown in the legends of the figures.

The repeatability results of this stage is also presented in Figure 3.22. What is signified in this figure is the fact that automated charging repetitions produce the 


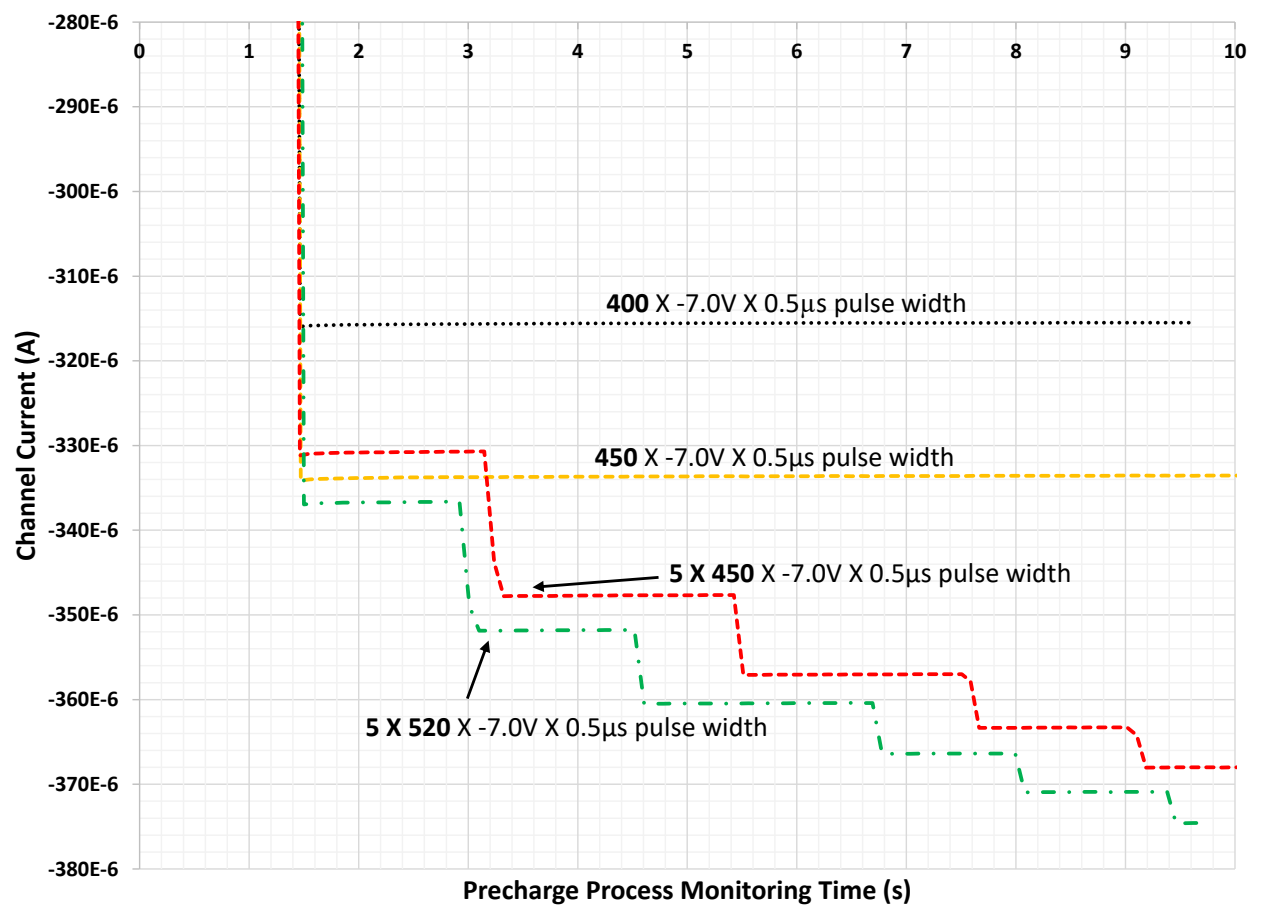

Figure 3.21: Precharging process of an FG-MOS. Numbers in bold represent the number of pulses in a pulse train applied to the shorted PMOS precharger device.

same amount of current every time.

To summarize, in order to precharge the gate (tunnel electrons onto the FG) negative pulses of $0.5 \mu$ s with a pulse height of $-7.0 \mathrm{~V}$ is required, and similar pulse height and width but in opposite polarity is needed to discharge the gate.

\subsection{Sub-threshold MOSFET Dosimeter}

In this section, a novel concept is introduced which utilizes the MOSFET in its subthreshold region as radiation dosimeters. The undesirable circuit complexity inherent to floating-gate MOS sensor precharging, and charge disturbance due to surrounding circuit blocks and human interactions drives the research in non-floating-gate (gateconnected) MOSFET dosimetry in this thesis. 


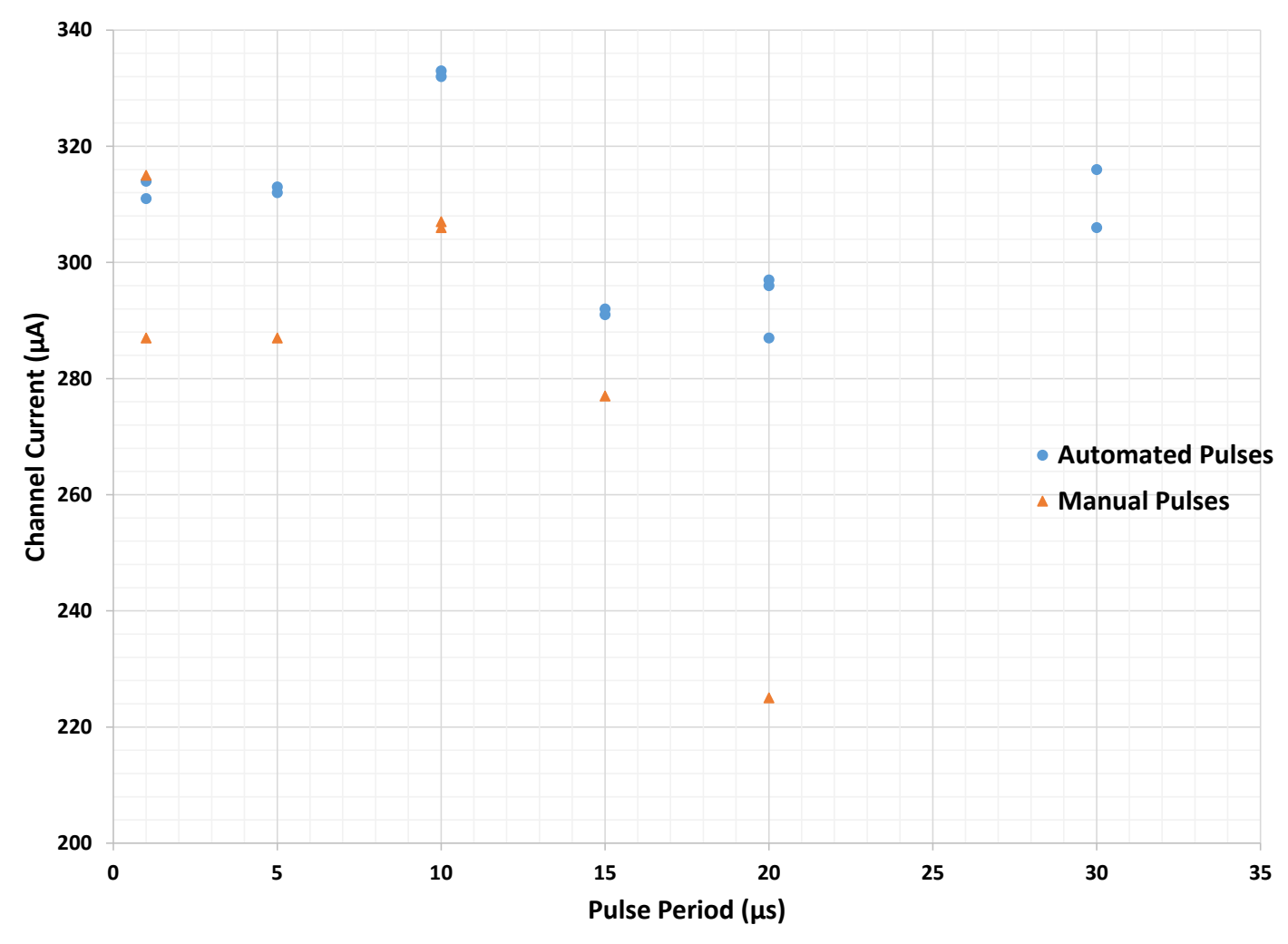

Figure 3.22: Precharging repeatability

MOSFET dosimeters developed in [85-88] prior to the development of floating-gate devices rely on the traps created by ionizing radiation in the thick gate oxide to shift $V_{t h}$ and hence measure the change in the channel current. This change in current is a measure of the cumulative dose absorbed in the oxide. This system requires a gate bias of $5 \mathrm{~V} \rightarrow 20 \mathrm{~V}$ depending on the MOSFET characteristics and the desired sensitivity. The higher the bias, the higher the sensitivity. Such MOS devices were used mostly to measure high energy photons in the $\mathrm{MeV}$ range employed in radiotherapy applications. Higher energy photon tends to pass through the oxide layer without interaction (electron-hole pair or trap generation) unless a high electric field is applied across the oxide to decrease the rate of recombination. Moreover, these MOSFETs are usually off-the-shelf components which cannot be integrated into a system-on-chip due to their large gate bias requirement. 
In this thesis, photon energy range of $\leq 100 \mathrm{keV}$ is used which increases photon interaction with oxide. Also, greater dose rates than in any radiation therapy application $\left(\geq 5 \mathrm{~Gy} \mathrm{~min}^{-1}\right)$ is used to irradiate blood which means lower sensitivity is also acceptable. This allows lowering the gate bias in order to lower the power consumption which is pivotal in the design of ultra-low power RFID sensor design.

In this section, two specific sub-objectives related to sub-threshold MOSFET dosimeter are pursued:

1. The study and design of sub-threshold device configurations;

2. Fabrication and experimental validation of the said designs.

\subsubsection{Principle of Operation}

Sub-threshold circuits are designed based on the simple concept of decreasing the supply voltage in order to reduce the energy consumption. The dynamic energy and power consumption of a CMOS circuit are governed by [89]

$$
\begin{gathered}
E=C V_{D D}^{2} \\
P=C V_{D D}^{2} f
\end{gathered}
$$

where $C$ is the total capacitance and $f$ is the frequency of operation.

As the above formulas suggest, lowering $V_{D D}$ has a subsequent squared decrease on the total dynamic energy and power. This is the main reason why subthreshold circuits are a subject of interest for Ultra-Low Power (ULP) applications. Some of these applications include wireless sensors, implantable devices/networks, ambient intelligence, wearable computing, smart grids and plant monitoring and static random 
access memories (SRAM) [90]. Another significant benefit of subthreshold circuits is their decreased leakage current and static power loss. The static power consumption of a CMOS circuit, $P_{S T}$, is given by [89]

$$
P_{S T}=V_{D D} I_{\text {Leakage }}
$$

where $I_{\text {Leakage }}$ is the total leakage current of the circuit. Using smaller supply voltage values for sub-threshold circuits leads to a linear decrease in static power consumption. This holds true assuming an equal leakage current for sub-threshold and superthreshold circuits. However, the leakage current for subthreshold circuits is also decreased, resulting in further static energy saving.

Although subthreshold circuits are superior to superthreshold ones in terms of power consumption, they do have drawbacks and challenges. The main concerns with subthreshold design are low speed and high process variation. In this study, the feasibility of using MOSFETs in subthreshold in radiation dosimetry is examined, which is of primary interest so process variation and speed are not investigated.

The most important characteristic of the MOS device in sub-threshold is the channel current equation [37].

$$
I_{d s}=\beta v_{T}^{2} e^{1.8} e^{\frac{V_{g s}-V_{t h}}{n v_{T}}}\left(1-e^{\frac{V_{d s}}{V_{T}}}\right)
$$

where

$$
\begin{aligned}
& \beta=\mu C_{o x} \frac{W}{L} \\
& n=1+\frac{C_{d e p}}{C_{o x}}
\end{aligned}
$$




$$
v_{T}=\frac{K T}{q}
$$

As can be seen in Eq.3.8, current $I_{d s}$ has an exponential relation with both $V_{g s}$ and $V_{t h}$. This means that a small shift in threshold voltage due to radiation is amplified exponentially and reflected in $I_{d s}$.

Variants of MOS devices available in $0.13 \mu \mathrm{m}$ technology were laid out to prepare for irradiaiton as presented in the next section.

\subsubsection{Sub-threshold MOSFET Design}

Variants of both NMOS and PMOS transistors were laidout and fabricated as shown in Figures 3.23, 3.24, and 3.25. As can be seen in Figures 3.23 and 3.24, there are three variants of MOS devices laid out, presented in Table 3.1. The transistors are close to minimum size. It would have been beneficial if the transistors were much larger in order to increase their gate oxide volume for better capture of incident photons but the initial thought on the design was to keep the dimensions to a minimum in order to draw the least amount of current at lower bias voltages.

Table 3.1: Dimensions of MOSFETs fabricated for subthreshold irradiation experiments using the blood irradiator X-ray machine

\begin{tabular}{|c|c|c|c|c|}
\hline Device ID & NMOS & W/L $(\mu \mathrm{m} / \mu \mathrm{m})$ & PMOS & W/L $(\mu \mathrm{m} / \mu \mathrm{m})$ \\
\hline 1 & Thick Gate Oxide I/O & $0.5 / 0.4$ & Thick Gate Oxide I/O & $1.0 / 1.0$ \\
\hline 2 & Thin Oxide Low Power & $0.16 / 0.12$ & Thick Gate Oxide I/O & $0.5 / 0.4$ \\
\hline 3 & Thin Oxide Standard & $0.16 / 0.75$ & Thin Oxide Standard & $0.16 / 0.12$ \\
\hline 4 & Thin Oxide Standard & $2 / 0.12$ & Thin Oxide Standard & $0.16 / 1.0$ \\
\hline 5 & Thin Oxide Standard & $0.16 / 0.12$ & Thin Oxide Low Power & $0.22 / 0.12$ \\
\hline 6 & Thin Oxide Standard & $1.0 / 0.12$ & & \\
\hline
\end{tabular}

Radiation exposure test has been performed on these devices and the results will be explained herein. 


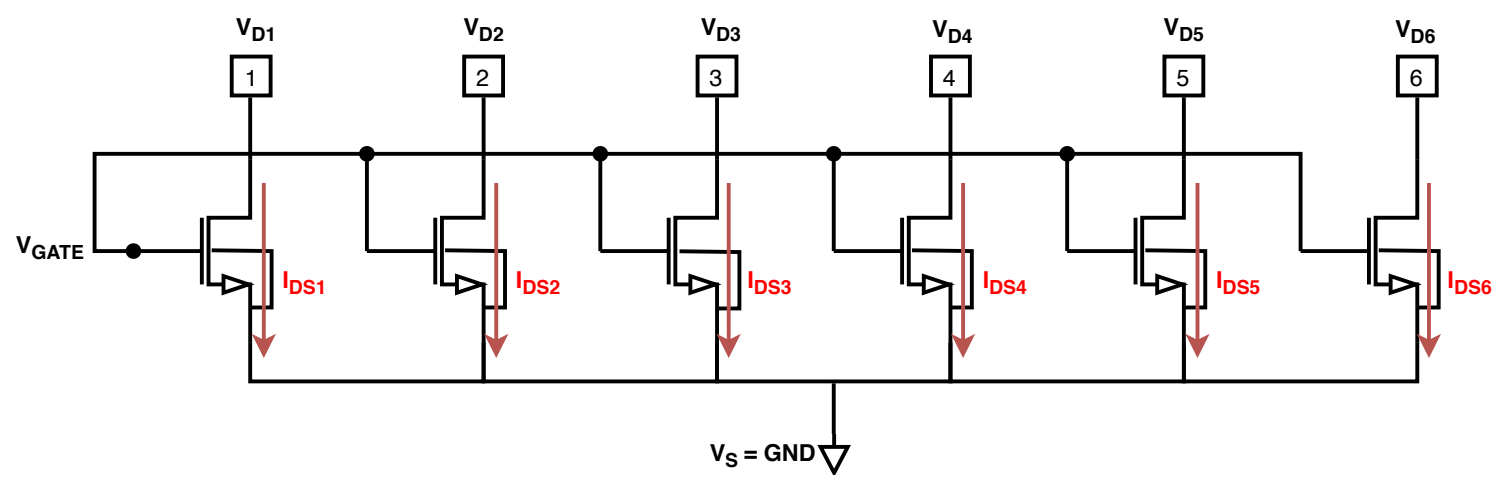

Figure 3.23: NMOS transistors fabricated

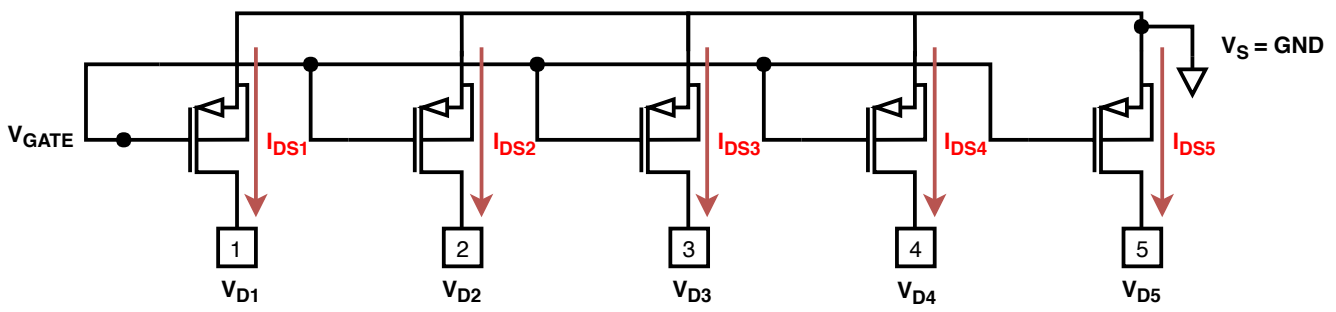

Figure 3.24: PMOS transistors fabricated

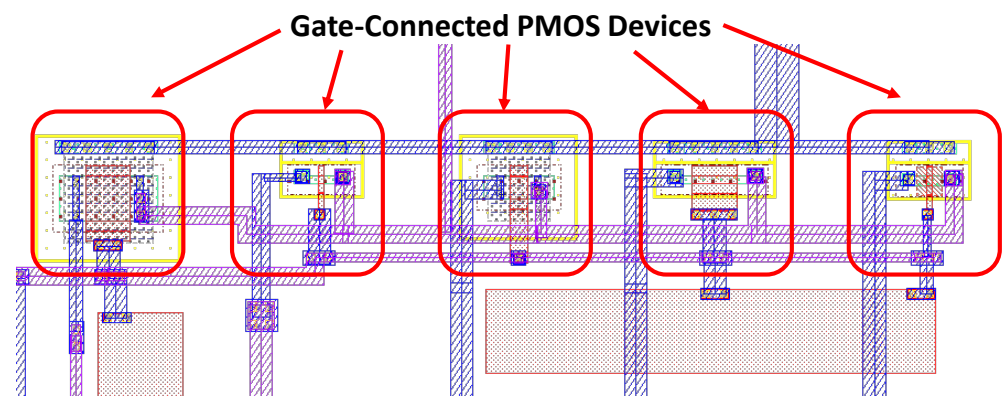

Figure 3.25: Sample Layout of fabricated PMOS devices

Another approach to a sub-threshold MOSFET radiation sensor design has been proposed. Referring back to Equation 3.8, another important factor in sub-threshold current of the MOSFET is the gate voltage which also has an exponential effect. A design proposed utilizes on-chip series chain of diodes which would develop a voltage under radiation across them. Once the chain of diodes is connected to the gate of the 
MOSFET, it biases it in sub- or near-threshold region depending on the number of diodes in series. A small drain/source bias at the MOS device results in current in the channel which could be integrated to determine the amount of radiation dose. The same developed voltage under radiation across the diodes can be applied to a chain of inverters in a current starved ring oscillator configuration. A counter then can be employed to measure the frequency. Another approach is to embed a MOS transistor biased with the diodes in a current mirror to set the current, hence the frequency of oscillation in the ring oscillator. An example of a voltage-controlled ring oscillator is shown in Figure 3.26.

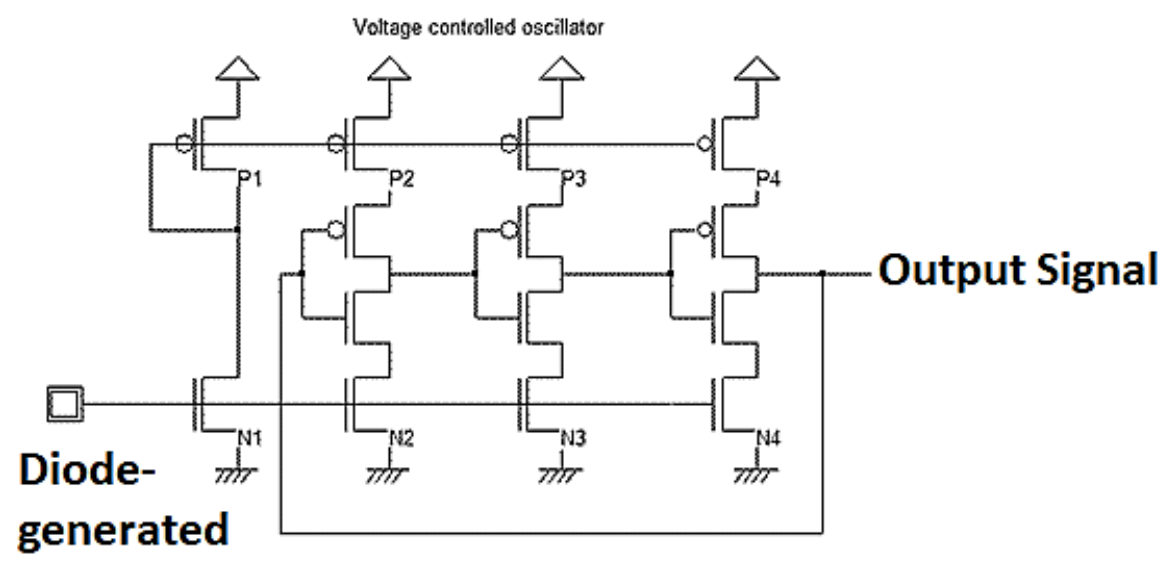

Figure 3.26: Voltage-controlled ring oscillator schematic

Figure 3.27 shows the irradiation results of a sample gate-connected NMOS device. The same irradiation conditions were applied (Blood irradiator machine, same ambient temperature, pressure, and duration) to the devices mentioned in Table 3.1. Preliminary experiments showed that low gate biases in the range of sub-threshold values between $\approx 0.3 \mathrm{~V}$ to $0.5 \mathrm{~V}$ would not result in any change within the 300 seconds of irradiation. Therefore, it was decided to increase the gate voltage to $1.0 \mathrm{~V}$. This already goes against the sub-threshold idea, but the experiments are nonetheless valuable. 
In the first 300 seconds of Figure 3.27, irradiation is happening and from that time onwards the rest period is presented. The rest period is there to monitor the behavior of the device immediately after irradiation to confirm whether there is any significant changes due to radiation exposure. The device was never physically moved between the two time period and the plot shows one continuous operation.

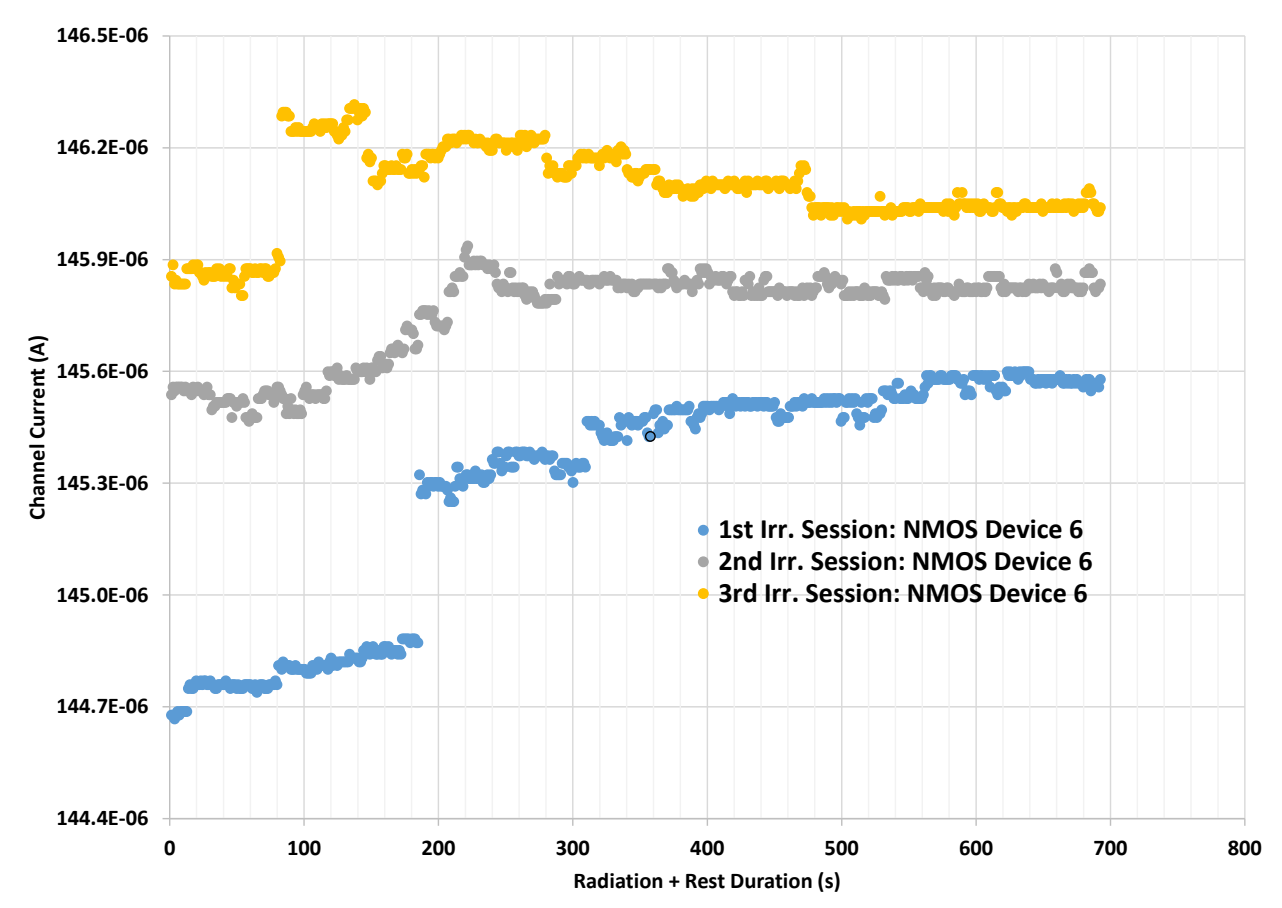

Figure 3.27: Device\#6 channel current change as per radiation

As can been seen from the figure, there is insignificant change in current during each of the three irradiation sessions in this device. This change could even be attributed to temperature changes within the device due to current draw or the X-ray machine heating up. Overall, the change is less than $1 \%$. Also, at a $V_{\mathrm{DS}}=1.0 \mathrm{~V}$, the power consumption is already more than the FG-PMOS case which consumed $\approx 30 \mu \mathrm{W}$ at its highest current draw which shows this solution might not be favored due to lack of radiation response and increased power consumption.

Unfortunately for the PMOS devices, the same lack of sensitivity to ionizing radiation 
from the blood irradiator machine was observed. Hence their measurement plots are not presented.

\subsection{Chapter Conclusion}

The main contribution of this chapter has been the study of the design and implementation of different RF-CMOS configurations of FG-MOSFET to understand the best possible design for the proposed radiation dosimetry application. Performance parameters such as sensitivity, linearity and power consumption have been kept in mind. The conclusions of this chapter are as follows:

1. A parametric study is carried out on PMOS FG devices in the presence and the absence of a passivation layer. Cobalt Silicide $\left(\mathrm{CoSi}_{2}\right)$ was used as the passivation layer on top of the FG extension. It is observed that the presence of silicide reduces the sensitivity of the FG MOSFET as compared to the devices which do not have these passivation layers. These results indicate the use of silicide in this application as a downside.

2. Similar to the last point, a study is carried out on the use of a grounded metal protection layer on the top of such sensors. The results thus obtained show that these metal layers do not have any effect on the device performance. But since the metal layer could still shield the sensitive floating gate from electromagnetic interference, its use is still relevant.

3. Finally, as a device variation, multiple gate fingers were employed instead of using a single gate finger while maintaining the same channel dimensions of the MOS transistor. In this case, like the last one, no significant change in the power consumption, sensitivity or linearity of the sensor was observed. 
4. The sensor devices are also characterized for the charging and discharging patterns using square pulses. To summarize, in order to precharge the gate (tunnel electrons onto the FG) negative pulses of $0.5 \mu$ s with a pulse height of $-7.0 \mathrm{~V}$ are required, and similar pulse height and width but in opposite polarity are needed to discharge the gate. Thus, these pulses can be applied when a sensor is required to be charged or discharged depending upon the scenario.

5. At the end of this chapter the sensors are tested for their operation under subthreshold bias conditions. Such an option is explored to study the viability under extremely low power conditions. Although the sensors do provide some sensitivity, the value is low and hard to read. Therefore, the next chapter investigates better techniques to address the challenges of low power operation. It will also be seen if the sub-threshold operation can be used in conjunction with another solution to improve the overall response. 


\section{Chapter 4}

\section{Integration and Functionality}

\section{Enhancements}

In the previous chapter it is shown how RF-CMOS technology can provide a solution as an FG-MOS sensor in radiation dosimetry. However, the design can be further improved by lowering the power/energy requirements. A new idea of sub-threshold operation was therefore investigated. In this chapter, the following novel approaches will be studied to reduce the power requirements or provide an efficient energy source:

1. N-Type Floating-gate MOSFET Radiation Dosimeter. NMOS devices have never been researched on and investigated in this $0.13 \mu \mathrm{m}$ RF-CMOS technology. It is research-worthy to implement NMOS floating-gate devices and evaluate their sensitivity, linearity and response reproducibility under irradiation.

2. Floating-gate Minimum Size Inverter Design. Novel minimum size shared floating gate inverter sensor is designed, implemented, characterized and irradiated as a digital sensor, consistent with the aim of low power sensor design.

3. Radiation-Powered Dosimeter Idea. Preliminary investigations and design 
of a novel device has been done, which involves on-chip harvesting of ionizing radiation for powering the dosimeter chip. This idea has not been explored and to the best of the author's knowledge, no publication has been presented on the topic.

\subsection{A Versatile FG-MOS Design}

The design presented in Figure 4.1 is a new versatile design. The PMOS in the middle is shorted and can be compared to the precharger device in the previous designs presented. The other two transistors on either side can be biased to measure the currents through them. A radiation response (current change vs radiation) can be obtained from the two to compare their behaviours under radiation. This means that the floating gate can be precharged by electrons to use the left PMOS as the sensor, or by holes to use the NMOS on the right as the sensor. Moreover, if the nodes $D_{P}$ and $D_{N}$ were to be tied together, and $V_{D D}$ be applied to $S_{P}$ while $S_{N}$ is grounded, then an inverter is obtained which could sense radiation by "flipping" its output from $0 \rightarrow 1$ or vice versa.

The shorted PMOS, like the previous case, has a gate oxide thickness of $5.2 \mathrm{~nm}$ as

reported by the foundry, and has $\frac{W}{L}=\frac{1.5 \mu \mathrm{m}}{1.4 \mu \mathrm{m}}$, while the other two have the dimensions $\frac{W}{L}=\frac{0.5 \mu \mathrm{m}}{0.4 \mu \mathrm{m}}$ making them minimum standard sizes with the same gate oxide thickness. The floating gate is wrapped around the shorted PMOS and the two transistors are placed on either side as seen in Figure 4.1(c). The dimensions of the entire cell is $14 \mu \mathrm{m} \times 7 \mu \mathrm{m}$.

The objective of this design block is to investigate whether a very small floating gate extension area over the shallow trench isolation (STI) can be enough as a charge 


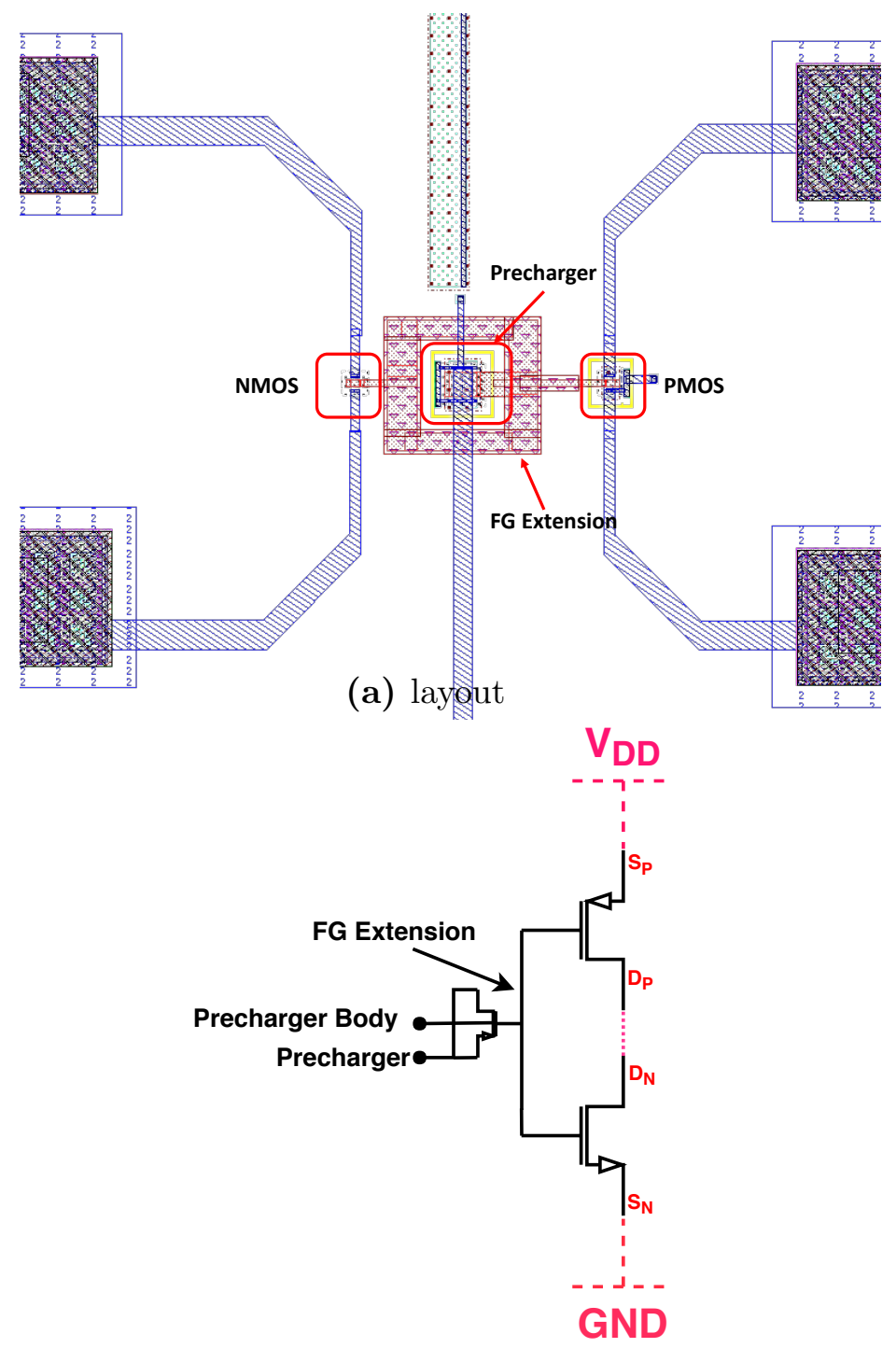

(b) schematic

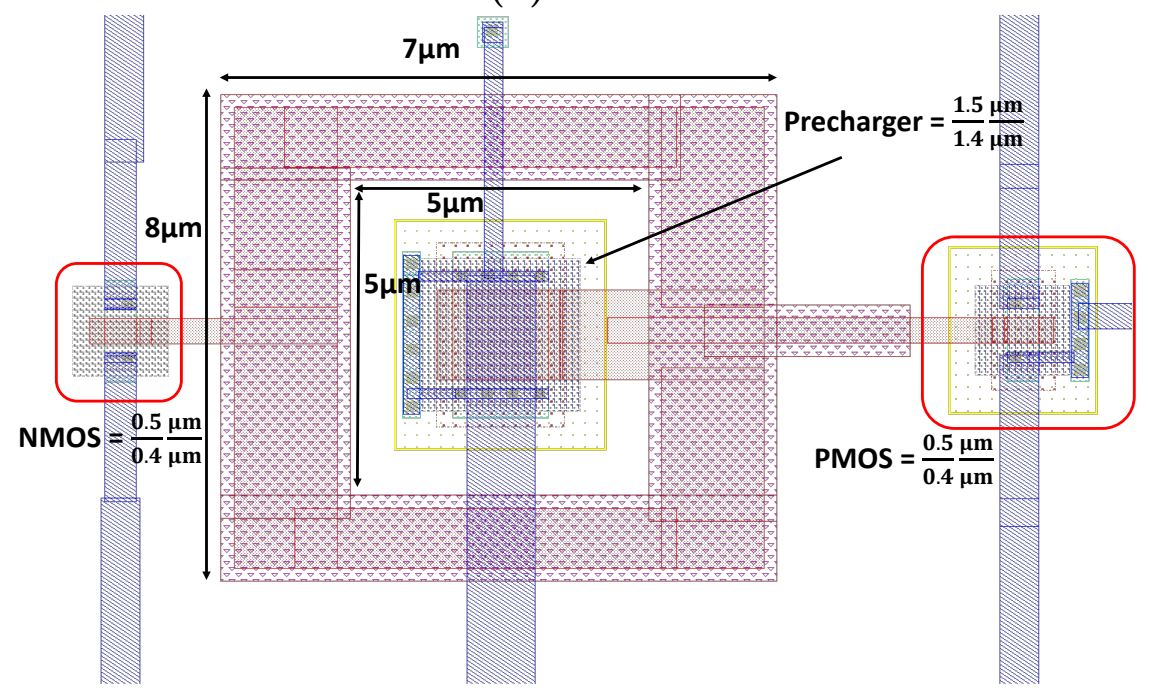

(c) Zoomed-in layout

Figure 4.1: Versatile floating-gate cell, (a) overall structure, (b) schematic, (c) zoomed-in layout 
collection body for the same radiation modes $(110 \mathrm{kVp}$ and blood irradiator X-ray sources) compared to the large FG-PMOS case studied in Chapter 3. Moreover, in the case of this block being sensitive to radiation, it is important that the block be precisely characterized in terms of its response reproducibility and power consumption. There is great potential in this design since it could potentially be used as a secondary solution to the single large PMOS devices previously studied.

Studying this cell would also allow an experimental comparison of the radiation sensitivity to previous designs in terms of MOS and floating extension sizes.

The original intention of this design was to be able to separately study the radiation sensitivity of the minimum size FG-PMOS as well as the FG-NMOS device. An issue encountered with process-related Design Rule Checks (DRC's) stops negative pulses to precharge the floating gate with excess electrons. The mandatory $n$-tie-down is a highly doped N-type (an $\mathrm{n}+$ ) region within the p-type substrate required for creating a discharge path for excess charge generated during the fabrication process in the PMOS regions. It is the reason why negative pulses cannot cause tunneling since the $n$-tie-down conducts; hence the FG-PMOS cannot be precharging. Instead, the NMOS device, which also shares the same floating gate can be precharged with excess holes and hence turn on prior to irradiation. As a result, numerous FGNMOS irradiation characteristic curves are available, but unfortunately, none for the minimum size FG-PMOS device.

The small NMOS and PMOS devices mentioned above also have their gate-connected counterparts of the same dimensions (shown in Figure 4.11) which were fabricated to enable direct I-V characteristic analysis of the devices. Figure 4.2 shows the $I_{D S}$ versus $V_{D S}$ curves for the NMOS device. Figure 4.3 shows the $I_{D S}$ versus $V_{G S}$. 


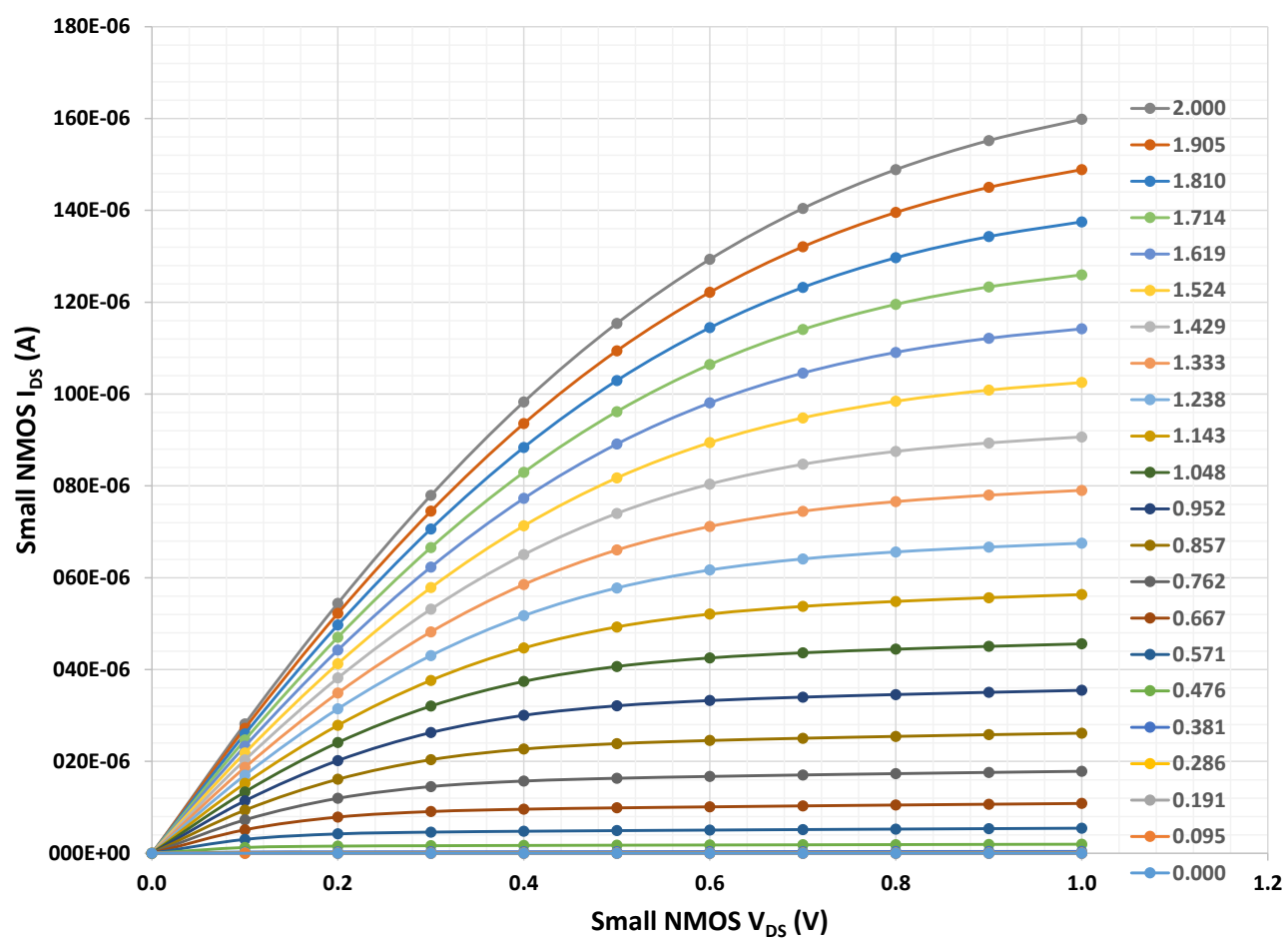

Figure 4.2: Small gate-connected NMOS $I_{D S}$ vs $V_{D S}$ curves (measured)

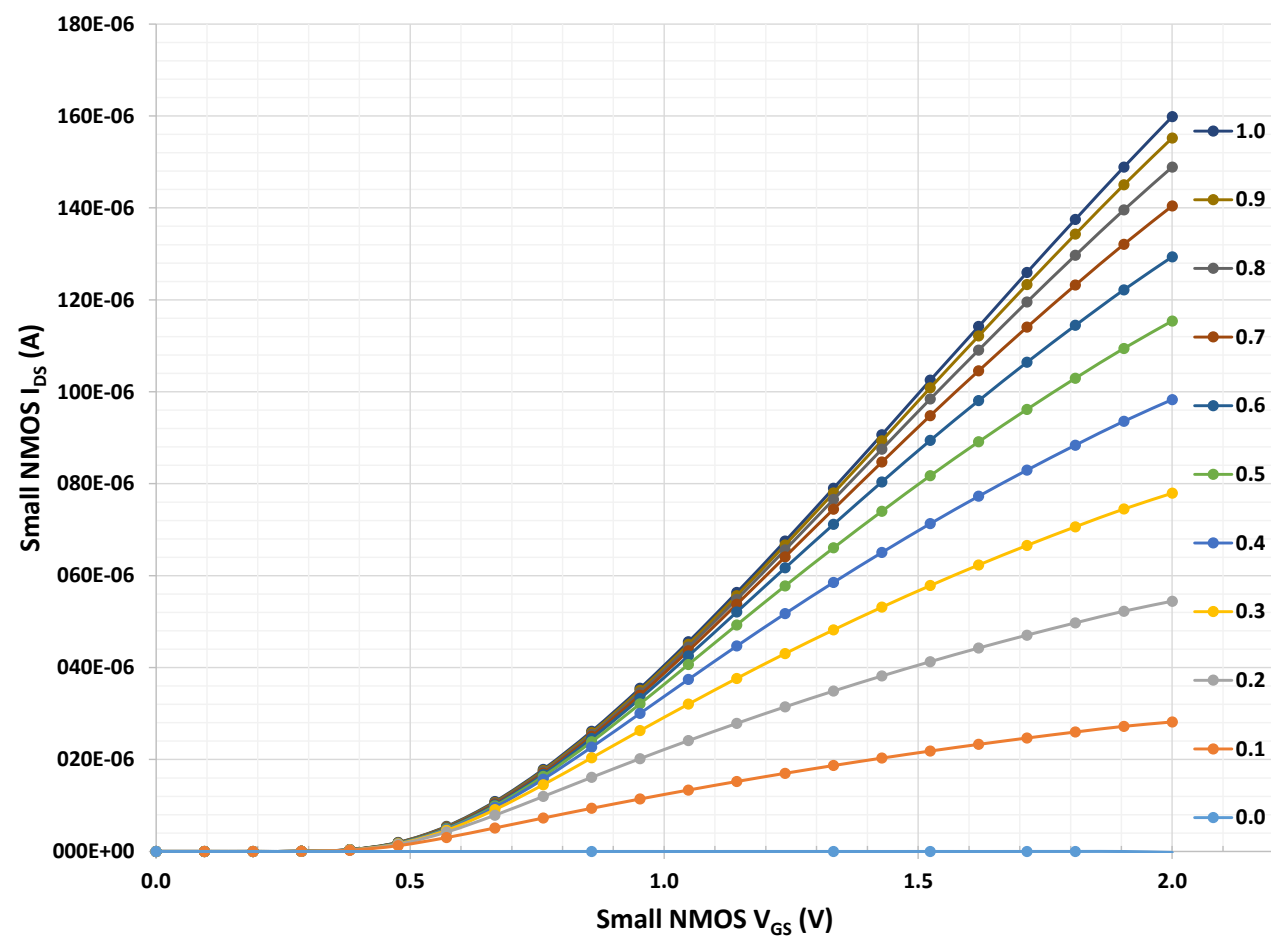

Figure 4.3: Small gate-connected NMOS $I_{D S}$ vs $V_{G S}$ curves (measured) 
Post layout simulation results are almost identical taking into account possible leakage paths, dark currents of junction diodes, and process variations.

Figure 4.4 shows the I-V characteristics of the small PMOS device.

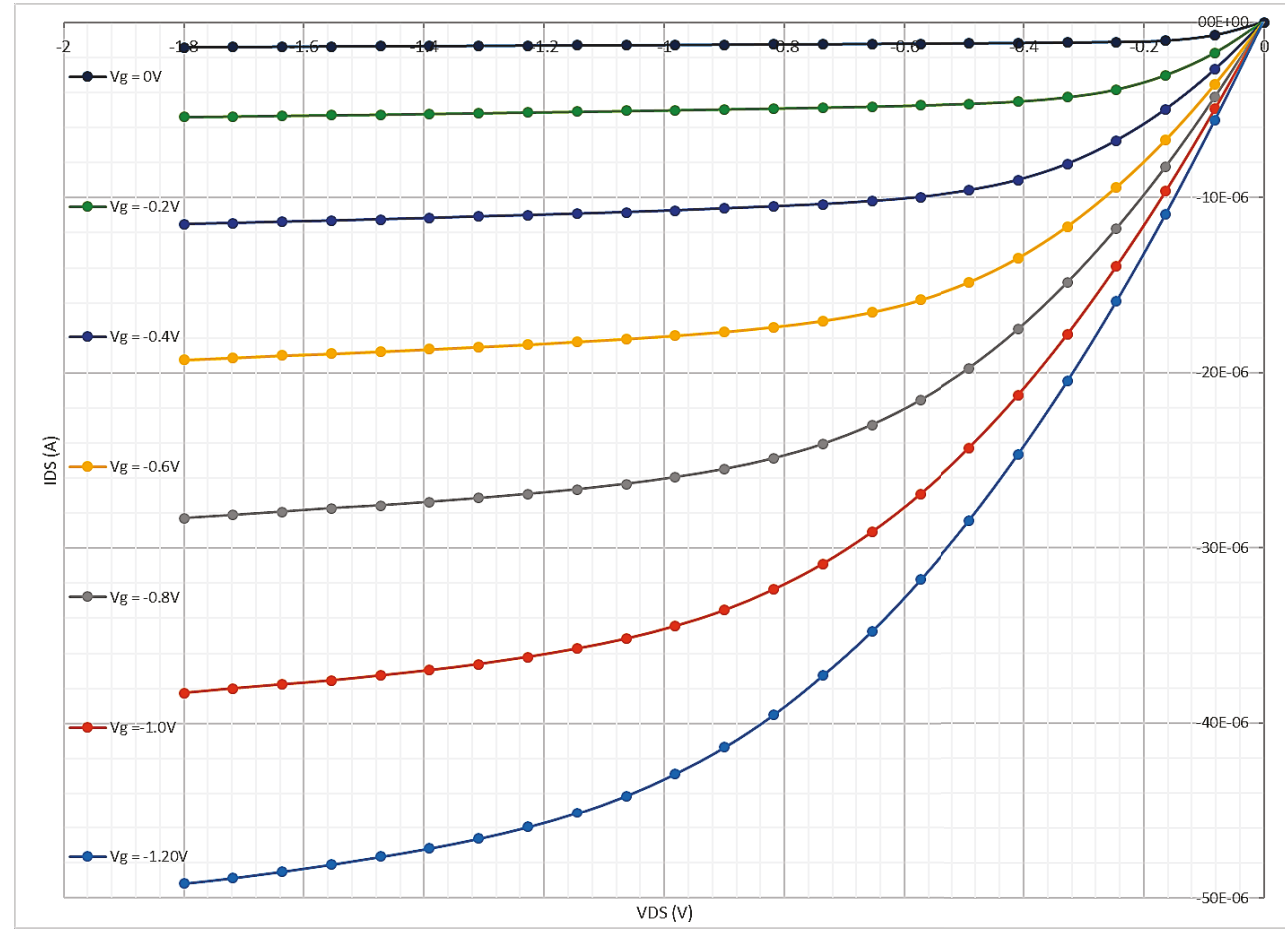

Figure 4.4: Small gate-connected PMOS $I_{D S}$ vs $V_{D S}$

Figures $4.2,4.3$, and 4.4 are included as complementary and a reference to clarify the upcoming points regarding the FG-NMOS and FG-inverter irradiation results. The first observation is the threshold voltages of the devices, which are $\approx 0.5 \mathrm{~V}$ and $-0.5 \mathrm{~V}$ for the NMOS and PMOS respectively.

Samples of the minimum size FG-NMOS irradiation results under both the $110 \mathrm{kVp}$ and the blood irradiator X-ray machine are presented in Figures 4.5, 4.6, and 4.7.

From Figure 4.2, it can be deduced that for an equivalent $V_{G S}=1.5 \mathrm{~V}, I_{D S}=100 \mu \mathrm{A}$ 


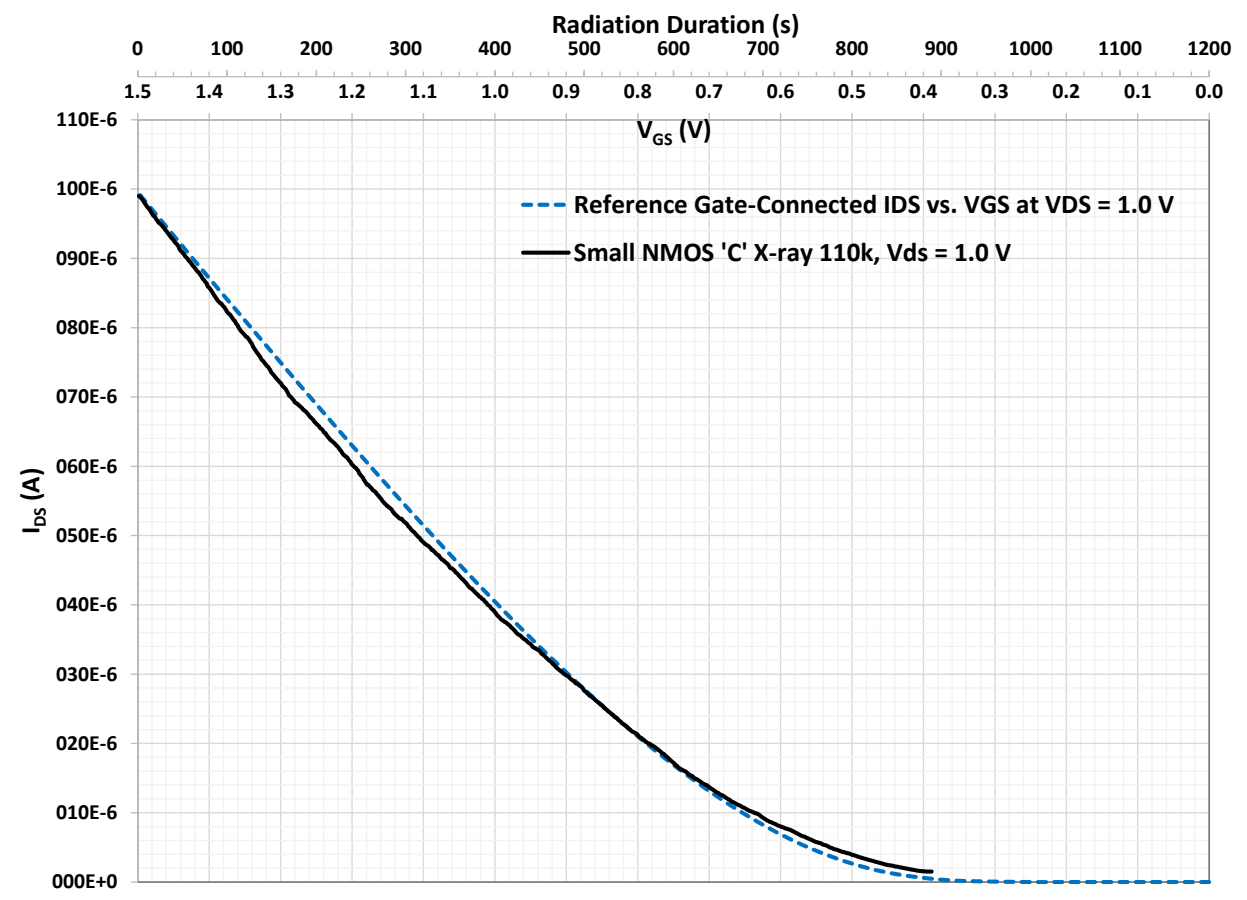

Figure 4.5: A sample of minimum size FG-NMOS device and its gate-connected reference biased at $1.0 \mathrm{~V}$ exposed to $110 \mathrm{kVp}$ X-ray

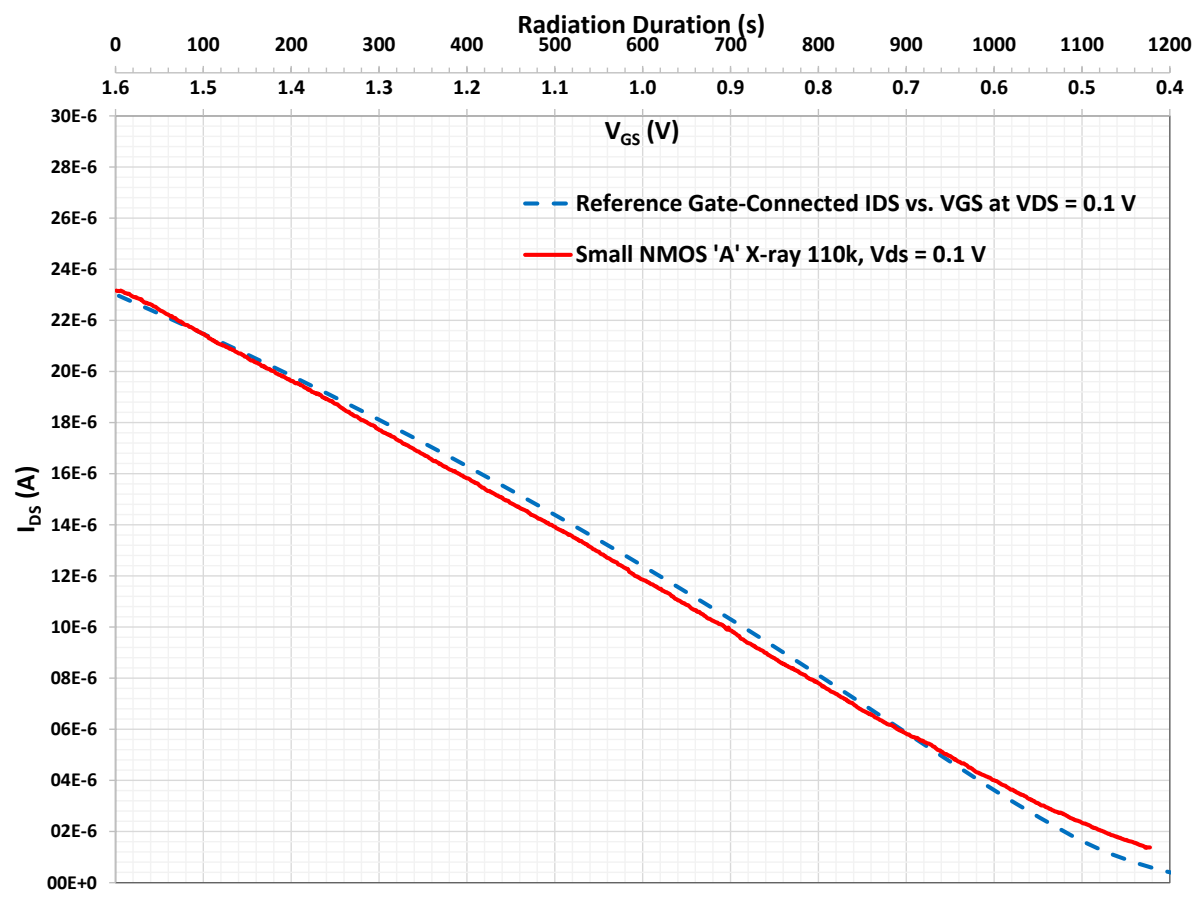

Figure 4.6: A sample of minimum size FG-NMOS device and its gate-connected reference biased at $0.1 \mathrm{~V}$ exposed to $110 \mathrm{kVp}$ X-ray 


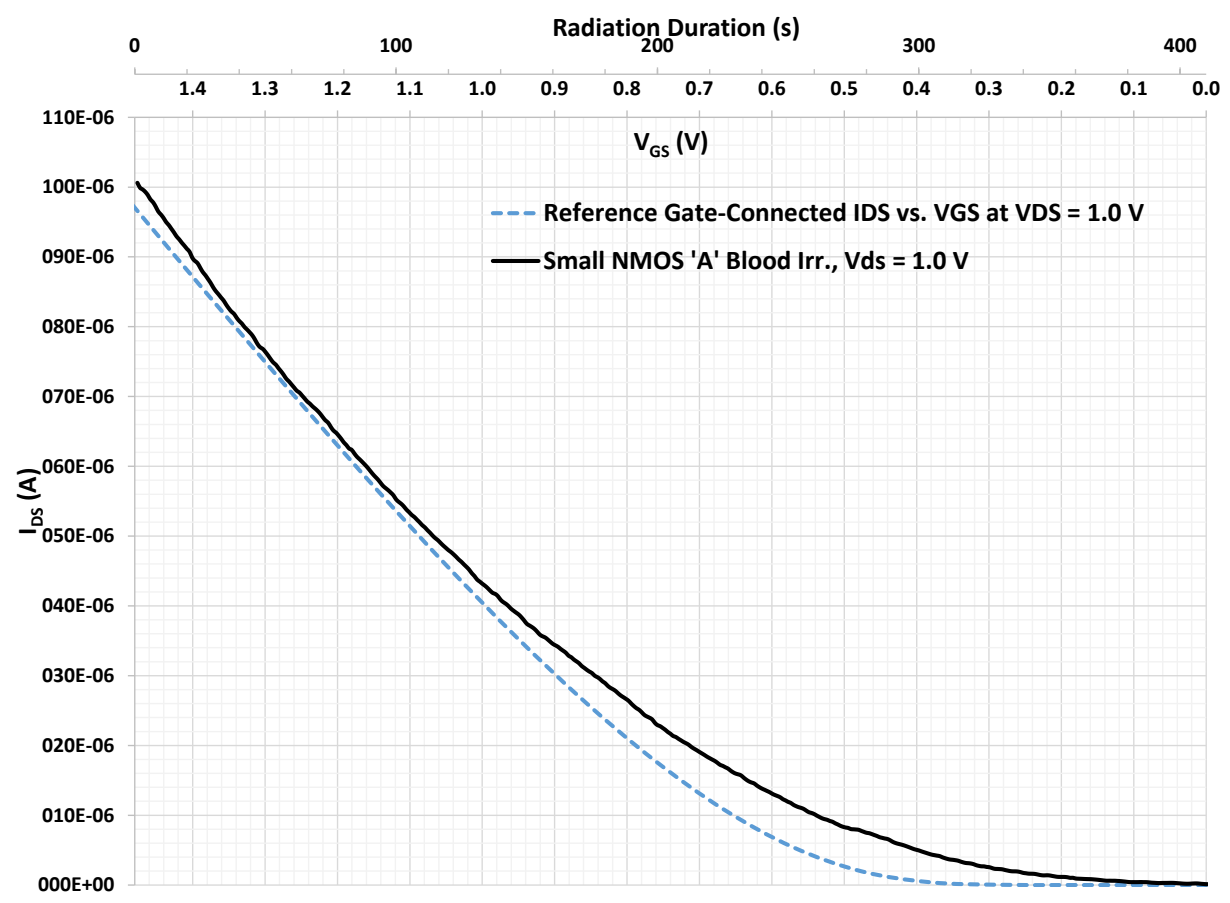

Figure 4.7: A sample of minimum size FG-NMOS device and its gate-connected reference biased at $1.0 \mathrm{~V}$ exposed to blood irradiator machine X-ray

at $V_{D S}=1.0 \mathrm{~V}$ (Figure 4.5) and for an equivalent $V_{G S}=1.6 \mathrm{~V}, I_{D S}=24 \mu \mathrm{A}$ at $V_{D S}$ $=0.1 \mathrm{~V}$ (Figure 4.6). This confirms the correct operation of the sample FG-NMOS devices since the sample presented in Figure 4.5 was precharged to the same level and operated at two $V_{D S}$ 's of $0.1 \mathrm{~V}$ and $1.0 \mathrm{~V}$.

In the case of $110 \mathrm{kVp}$ X-ray, irradiating the FG-NMOS demonstrates a linear relation between $I_{D S}$ and irradiation at $V_{D S}=0.1 \mathrm{~V}$ emphasizing that the NMOS is operating in the triode region of operation, whereas in the case of $V_{D S}=1.0 \mathrm{~V}$, a nonlinear relation exists $\left(2^{n d}\right.$ order polynomial) as in the saturation region of operation. Figures 4.5 and 4.6 also reveal another interesting fact and that is for the case of irradiation with the $110 \mathrm{kVp}$ X-ray source, $I_{D S}$ vs irradiation lies directly on the gate connected counterpart's $I_{D S}$ vs $V_{G S}$. The dotted lines in both of the figures show that regardless of $V_{D S}, I_{D S}$ always follows the reference (gate connected) curves. This is a useful conclusion that one can predict the irradiation behavior of the FG-NMOS device 
before irradiation in the case of lower photon flux and energy X-ray source. Moreover, the irradiation by the blood irradiator machine also matches the same curve at $V_{D S}=$ $1.0 \mathrm{~V}$ with the only difference being the faster discharge time compared to irradiation using the X-ray machine as shown in Figure 4.7.

Another observation is the fact that the FG-NMOS devices completely discharge and $I_{D S}$ reduces to 0 ampere within the irradiation duration using either of the X-ray sources as opposed to the FG-PMOS devices presented in the previous chapter. In this case, the FG-PMOS device could be used as a current source for some oscillating circuit such as a ring oscillator since it does not completely turn off, otherwise, the current to the ring oscillator would cut off causing it to completely stop oscillating.

The FG-NMOS device could potentially be used as a switching device since when precharged, some current corresponding to the amount of charge pass through the channel, and when irradiated, it completely stops conducting.

\subsection{Minimum Size Floating Gate Inverter}

Inspired by the results of the irradiation of the FG-NMOS devices, a new radiation sensing block is designed. As can be seen from Figure 4.1, it includes minimum size thick-gate $(5.3 \mathrm{~nm}) \mathrm{NMOS}$ and PMOS transistors connected to a small floating gate extension $(8.3 \mu \mathrm{m} \times 7.2 \mu \mathrm{m})$. The floating gate is precharged by applying pulses to the shorted PMOS in between the P and NMOS devices. As explained before, only the NMOS was able to be charged by tunneling the electrons out of the floating gate, causing an excess hole accumulation, hence turning on the NMOS device and turning off the PMOS.

In order to have an inverter, the terminals of the device shown in Figure 4.1b were 
tied to each other and the source of the PMOS was connected to $V_{D D}$ and the source of the NMOS to GND. The floating gate which is shown as a square loop around the control gate is shown in Figure 4.1c. Underneath the floating gate an STI of $350 \mathrm{~nm}$ is what makes this device sensitive to ionizing radiation as was the case for the FGPMOS device discussed in Chapter 3. Originally, the intension was to precharge the floating gate by applying positive pulses to the precharger shorted PMOS device, but unfortunately, fabrication issues did not allow for that; therefore, the PMOS device shown on the left hand side of Figure $4.1 \mathrm{~b}$ was used to apply the positive pulses. The floating gate was precharged by excess holes by applying positive pulses of $\approx 7.0 \mathrm{~V}$ while the NMOS device was biased at $1.0 \mathrm{~V}$ and the current being monitored. The proper connections were made after to configure an inverter out of the two devices while the precharger shown on Figure 4.1b was grounded. Since the NMOS is turned on prior to irradiation, the output of the inverter is at $0 \mathrm{~V}$.

The FG-inverter was simulated in Cadence Virtuoso ${ }^{\circledR}$ prior to fabrication and it was tested in the $V_{D D}$ range of $0.5 \mathrm{~V}$ to $1.0 \mathrm{~V}$ in all the extreme corners (fast-fast, slowfast, etc.) and in the range of temperatures from $0{ }^{\circ} \mathrm{C}$ to $85^{\circ} \mathrm{C}$. This was performed to investigate whether the switching voltage has a significant shift which could offset the results to an extent. While it would have been ideal to be able to test the devices in the mentioned temperature range under radiation, not having access to the gate to apply an external bias and not being able to change the temperature of the chips while being irradiated means that switching only occurs as a result of radiation and cannot be easily manipulated. The closest one can come to temperature range testing would be to either test regular gate-connected inverter block or trust the simulation results. Considering the evidence that the simulations and fabricated chip results follow each other very closely, it was decided to present the simulated transfer characteristics of the inverter but with a gate connection instead. Figure 4.8 shows the $\frac{V_{\text {out }}}{V_{\text {in }}}$ and power 
supply currents of an inverter with different corners and temperatures considered.

Temperature variations and process corners affect the transfer function characteristics as shown in the figure. A variation of $\approx 80 \mathrm{mV}$ in the switching point is observed, and switching point current draw can vary between $\approx 2.6 \mu \mathrm{A}$ and $340 \mathrm{nA}$. The important conclusion is that for a constant room temperature of $25^{\circ} \mathrm{C}$, process variations can shift the mid-point hence the inverter radiation sensor cannot have any resolution better than the process variation itself. This does not however discourage the investigation of this new design since for many applications such as blood irradiation indicators, food irradiation or surgical tools sterilizations, a considerable radiation dose is applied with a wide radiation measurement tolerance which makes this device a viable choice nonetheless.

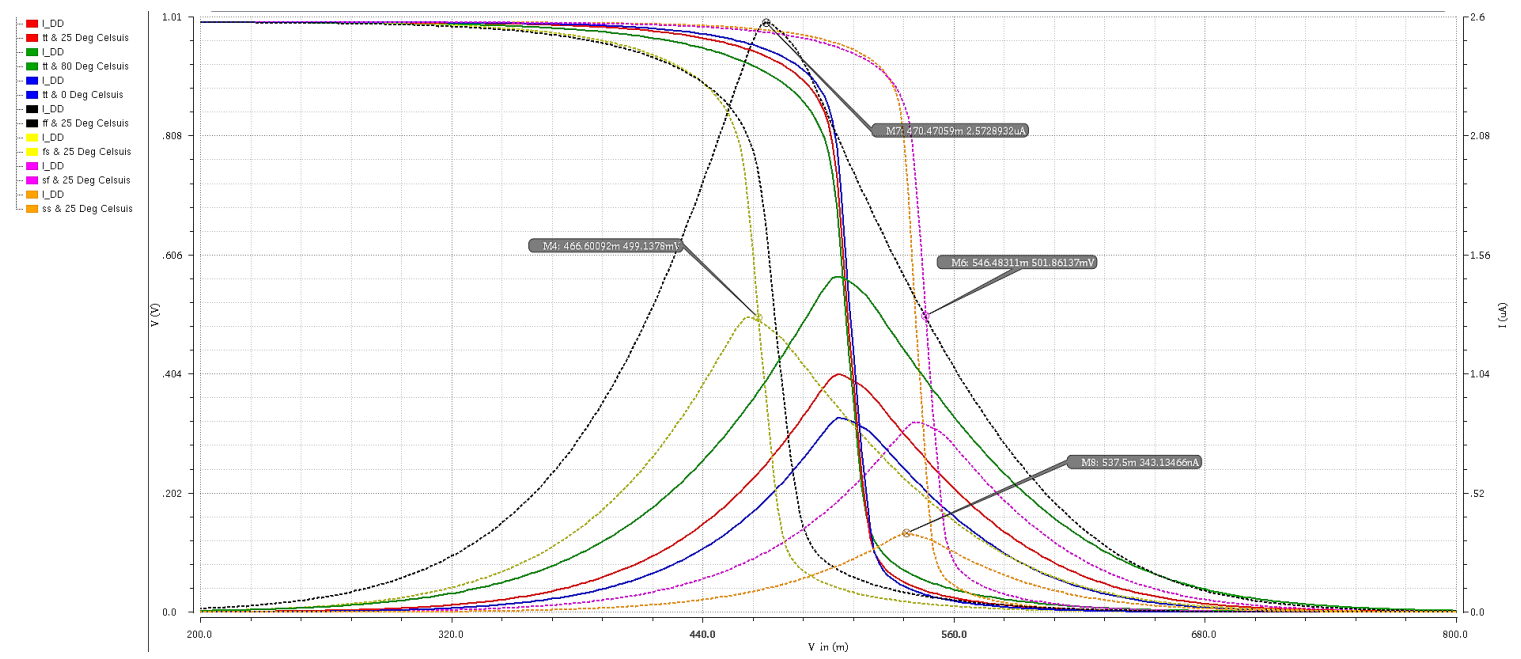

Figure 4.8: An inverter transfer characteristics in different corners and temperatures (simulation)

The behavior of the FG-inverter devices were investigated under radiation using the blood irradiator machine. Considering the goal of designing low power radiation sensors, the next logical step is to vary the power supply of the circuit block from $0.5 \mathrm{~V}$ to $1.0 \mathrm{~V}$ 
Figure 4.9 presents the FG-inverter biased at $0.5 \mathrm{~V}$ under radiation using the blood irradiator machine.

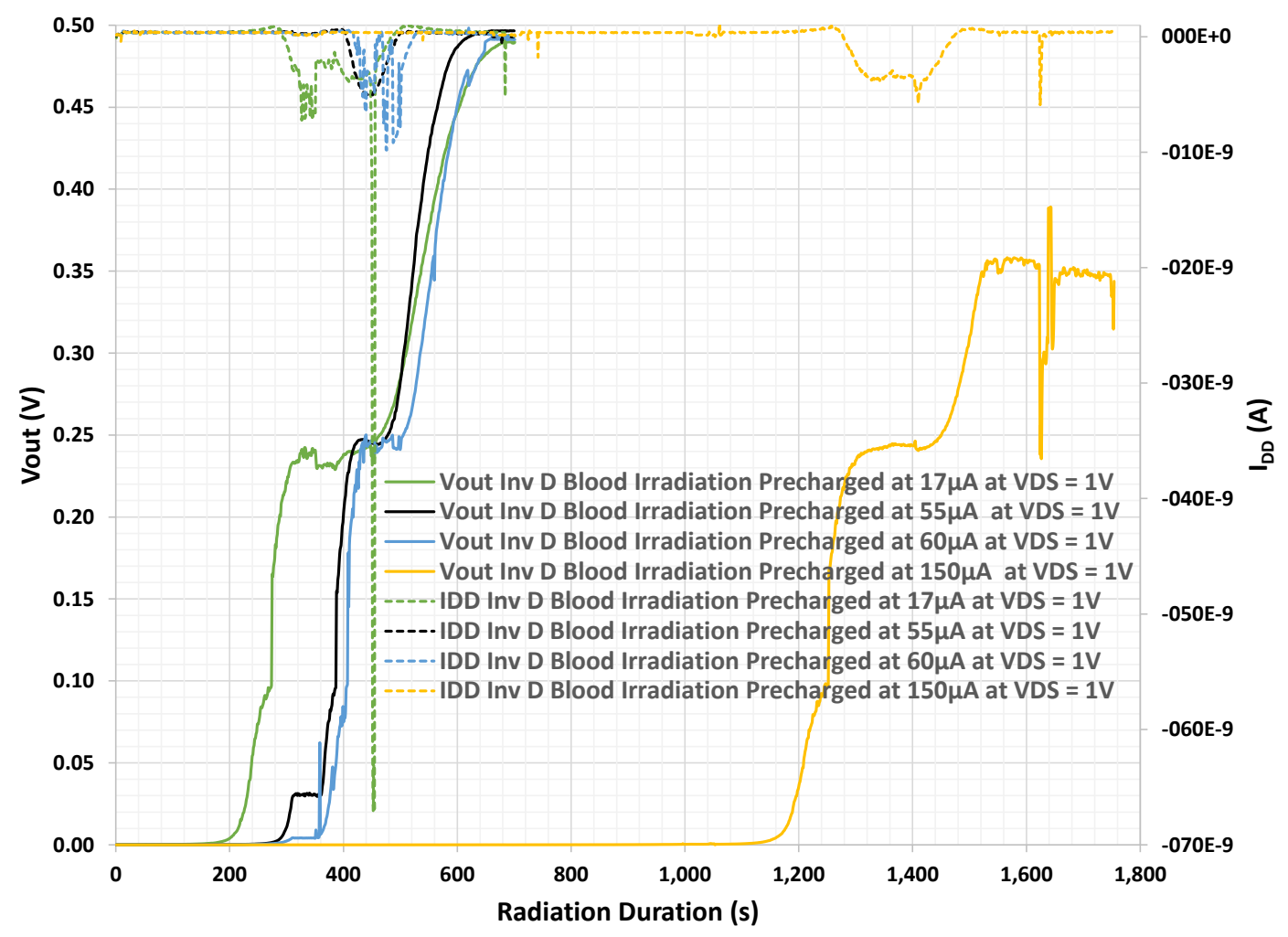

Figure 4.9: Radiation behavior of an FG-inverter biased at $0.5 \mathrm{~V}$ supply in the blood irradiator machine

The dotted lines represent the current draw from the supply and the solid lines show the output voltage behavior of the inverter. It can be seen that the transfer characteristic curves resemble that of an inverter but not exactly. Prior to irradiation, the floating gate is precharged with excess holes which turns on the NMOS and turns off the PMOS device; hence, the output voltage is at $0 \mathrm{~V}$ to start. During irradiation, the excess holes recombine with the radiation induced electrons from the electron-hole pairs which consequently weaken the NMOS and starts to turn on the PMOS. As the NMOS channel current reduces, the subthreshold current in the channel of PMOS causes the output voltage to rise to $V_{D D}$. This overall behavior can be observed from 
the figure. However, as seen in Figures 4.2, 4.3, and 4.4, both the N- and PMOS devices have $V_{t h} \approx \pm 0.46 \mathrm{~V}$. This means that initially, the NMOS has an equivalent gate voltage of $\approx 0.8 \mathrm{~V} \rightarrow 1.9 \mathrm{~V}$ (for the $I_{D S}$ range of $17 \mu \mathrm{A} \rightarrow 150 \mu \mathrm{A}$ ) deduced from Figure 4.3 , which puts it in the triode region of operation $\left(V_{D S_{n}}<V_{G S_{n}}-V_{t h}\right)$ while the PMOS is OFF. As radiation discharges the floating gate, the equivalent $V_{G S}$ of the NMOS reduces until it moves to the subthreshold region of operation which makes it a high resistance. Meanwhile, the PMOS does not still turn on since the equivalent $V_{G S_{p}}<V_{t h}$. This is exactly what is observed in the $\frac{V_{D D}}{2}$ region. In this region, both the NMOS and PMOS are essentially OFF and act as high resistance values. The current draw regardless of how insignificant is still present due to this leakage path. As radiation exposure continues, the floating gate further discharges and the PMOS's equivalent gate voltage gets closer to $V_{t h}$ which makes it a lower resistance than the NMOS; therefore, the output voltage rises to $V_{D D}$. It is also noted from the radiation tests shown in Figure 4.9 and many other experiments not shown, operating the inverter at $0.5 \mathrm{~V}$ results in unstable and unpredictable behavior under radiation. As seen in the 'yellow' curve in the figure, the output did not rise to $V_{D D}$ in one case of the exposure of the same chip labeled ' $\mathrm{D}$ ' in the figure. The valuable outcome of this experiment is the correlation of the amount of excess charge prior to irradiation and the point at which the inverter output "flips"; the more the excess charge, the further the inverter goes before changing the output under radiation. Experimentally, the desired point at which the inverter output flips can be set to coincide with the necessary amount of radiation for different applications. The design consideration here is that both the NMOS and PMOS were chosen to be their minimum size possible. In an ordinary inverter design, the ratio of the $\mathrm{P}$ to NMOS $\frac{W}{L}$ is chosen close to $\frac{2.5}{1.0}$ in modern transistors in order to offset the P-type device's lower charge mobility and hence set the mid-point of $V_{\text {out }}$ to that of $V_{i n}$. In the case of designing the inverter 
as a sensor, input to the sensor is total cumulative ionizing radiation incident on the floating gate and the bit flip can be set to occur at any desired point during the irradiation by manipulating the excess charge. Therefore, the main consideration is to minimize current draw in the mid-point (transition) region which directly relates to the size of the transistors. Any larger transistor width results in larger currents without any benefit to sensitivity. Supply voltages below $0.5 \mathrm{~V}$ were tested and the output voltage did not rise to $V_{D D}$ due to $\mathrm{P}$ and NMOS threshold voltages. Supply voltage was next raised to $0.7 \mathrm{~V}$ and then to $1.0 \mathrm{~V}$ as shown in Figure 4.10.

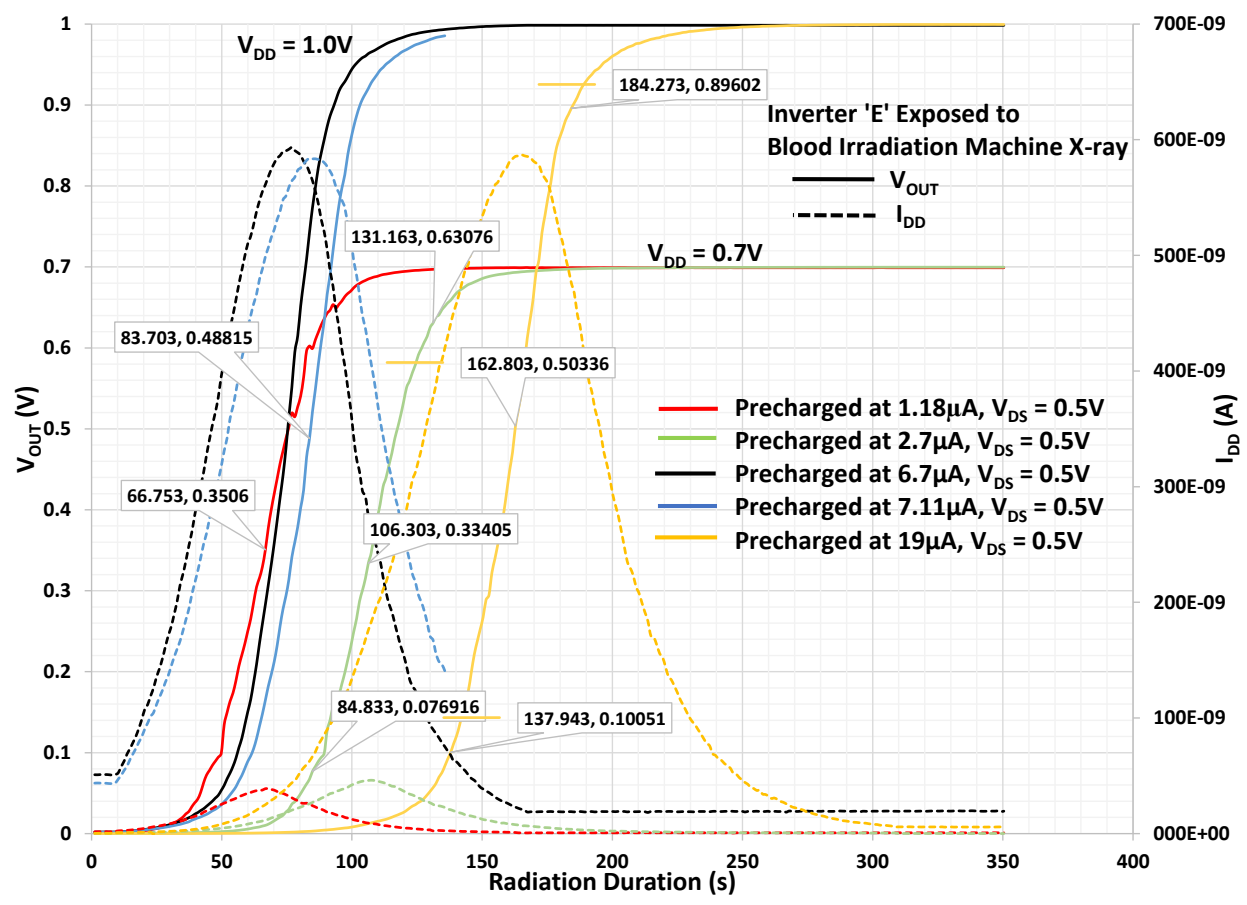

Figure 4.10: Radiation Behavior of an FG-inverter powered by $0.7 \mathrm{~V}$ and $1.0 \mathrm{~V}$ supplies in the blood irradiator machine

As seen in the figure, beyond $0.7 \mathrm{~V}$, the issue with lower supply voltages is not observed since both $\mathrm{N}$ and PMOS have enough supply to conduct better and with lower channel resistance even though they still go through the subthreshold region when the gate equivalent voltage drops below $0.5 \mathrm{~V}$ at which point $V_{G S_{p}}=0.2 \mathrm{~V}$ but it can still 
conduct more current to pull the output high without being stuck in $\frac{V_{D D}}{2}$. Also current consumption increases but not beyond $600 \mathrm{nA}$ which is perfectly acceptable since this means only nanoWatts of power consumption. Another important observation is that precise precharging of the floating gate is essential in having the inverter flipping the output at the right time. For example, if it is needed for the inverter to flip at or close to $35 \mathrm{~Gy}$ of radiation, regardless of the duration, the FG-inverter should be precharged at $\approx 40 \mu \mathrm{A}$.

Considering the same dose rate through the duration of the irradiation, it is also observed that precharging the device at a lower or higher charge does not change the rise time of the inverter response significantly. For example, for the test case shown in Figure 4.10 precharged at $19 \mu \mathrm{A}$ - 'yellow' curve-, the $10 \%$ to $90 \%$ rise time is $46.4 \mathrm{~s}$ and that of the sensor precharged at $2.7 \mu \mathrm{A}$-'green' curve- is $47 \mathrm{~s}$. The slight faster rise of the case at $V_{D D}=1.0 \mathrm{~V}$ is due to higher current drive of the inverter.

Another observation is that precharging the floating gate at different ratios in different cases with respect to each other does not directly translate to the same ratio in $50 \%$ times delay between the curves. For example, precharging the FG-inverter at $7.11 \mu \mathrm{A}$ and $19 \mu \mathrm{A}$ result in a mid-point of transitions of $84 \mathrm{~s}$ and $163 \mathrm{~s}$. The ratios $\frac{19}{7.11} \neq \frac{163}{84}$. This could be due to the non-linear relation between the NMOS $I_{D S}$ and the $V_{G S}$ as it goes through different regions of operation as it transitions the output.

Figures 3.23 and 3.24 are redrawn with some explanations in Figure 4.11. This section helps confirm some of the findings in the FG-inverter section by investigating the behavior of a gate-connected inverter under different bias and input conditions. Since the FG-inverter was tested with $0.5 \mathrm{~V}, 0.7 \mathrm{~V}$, and $1.0 \mathrm{~V}$ biases, the gate connected version will also be tested under the same conditions. In this case, since there is no radiation involved, the input to the inverter is just the voltage sweep. 


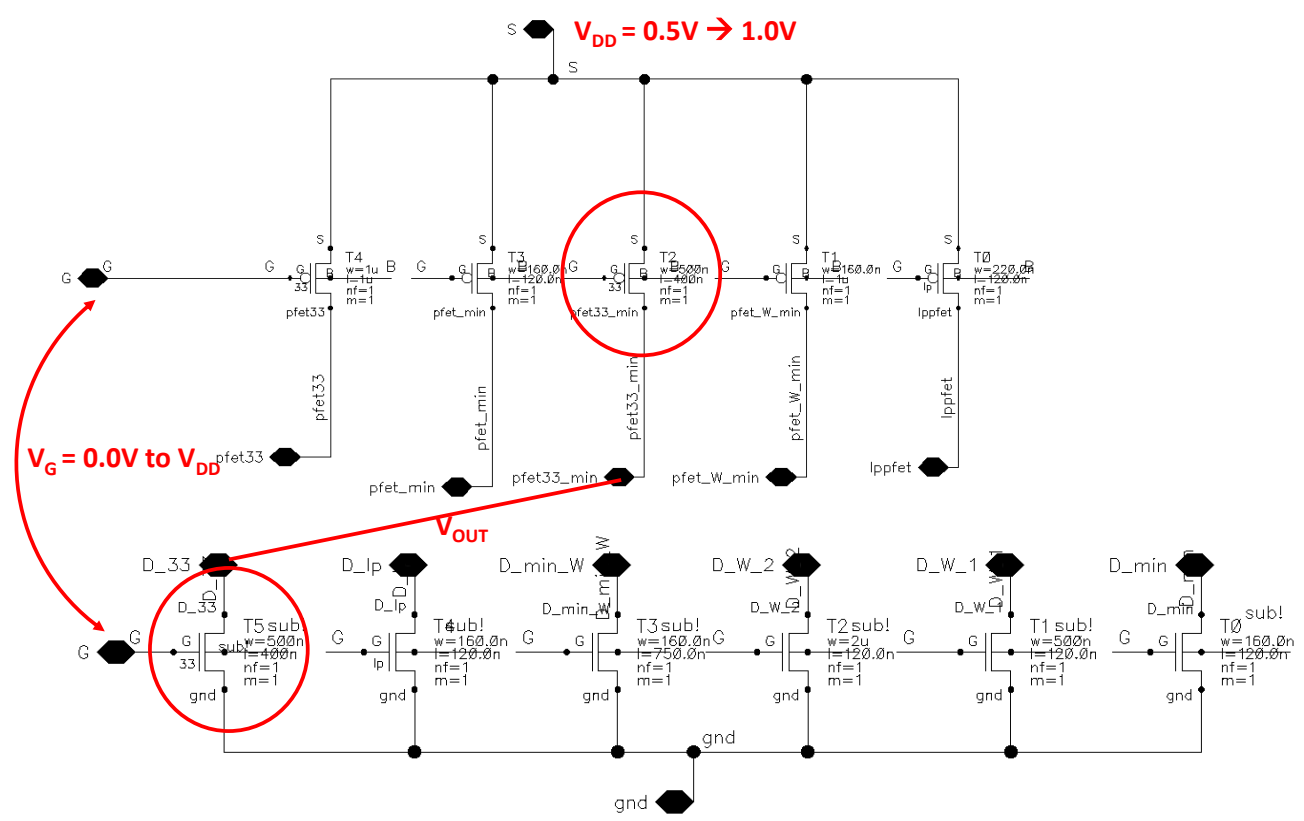

Figure 4.11: Identical minimum size $\mathrm{N}$ and PMOS devices externally connected to created the inverter

An $h p 4145 A$ Semiconductor Parameter Analyzer is used to apply and read voltages and currents. In addition to comparing the behavior of the FG-inverter with its gate-connected counterpart, a batch-to-batch comparison is also performed since the transistors shown in Figure 4.11 were fabricated a year prior to the FG-inverter chip. Figure 4.12 illustrates the measured values of an inverter at different $V_{D D}$ s corresponding with the curves from Figure 4.10.

Significant points to be made are the fact that $I_{D D_{V_{D D=1}}}=860 \mathrm{nA}$ which is higher than the FG-inverter case. This is due to the existence of ESD protection circuitry in the case of the gate-connected device which draws a DC current and also other leakage paths that unfortunately exist due to gate damage to a parallel connected device on chip which draws current through the gate electrode, hence lowering it which makes the devices not reach full $V_{D D}$. The current $I_{D D}$ shown however is the actual current 


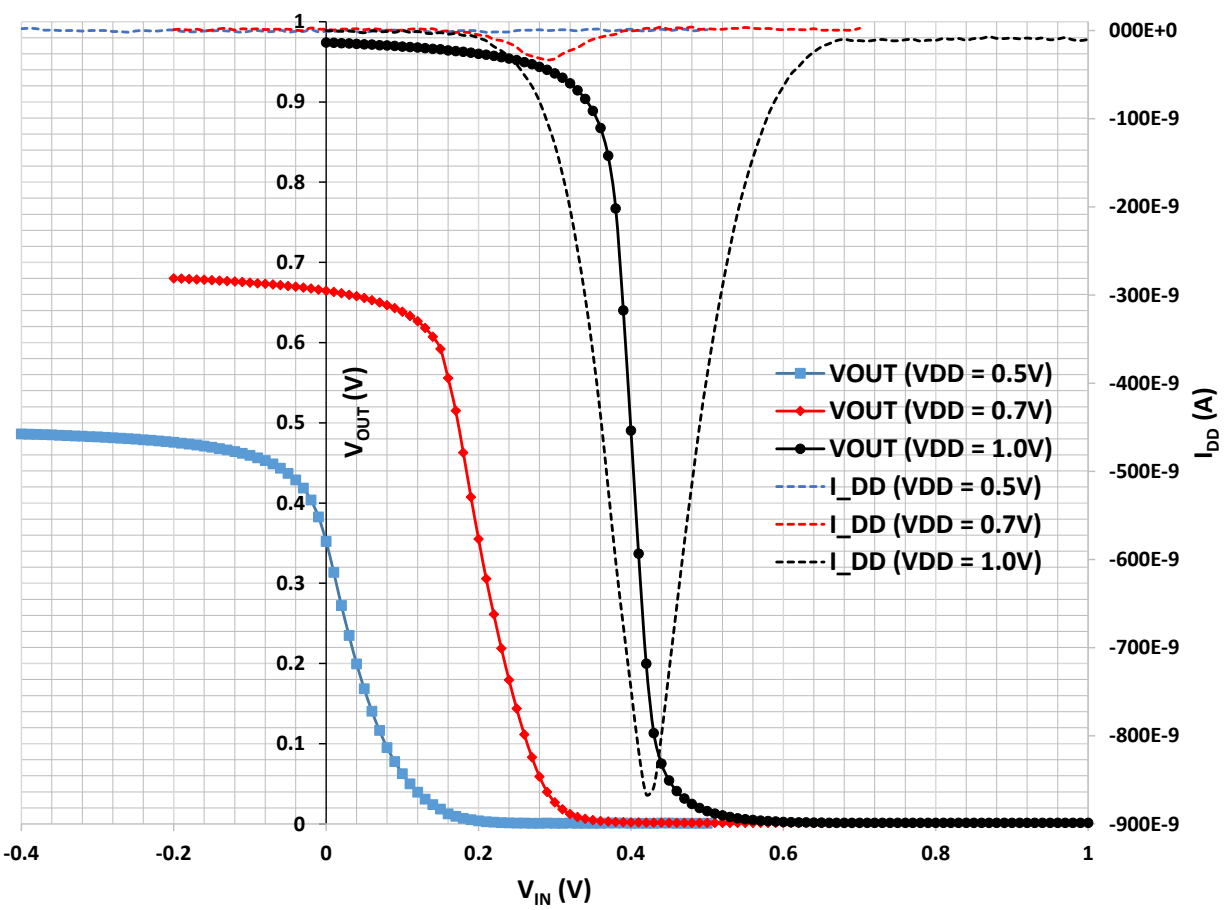

Figure 4.12: Gate connected inverters transfer characteristics and current consumptions at different $V_{D D} \mathrm{~S}$

running through the device and not that of the gate leakage.

Overall, it is concluded that using the FG-inverter device as a "1-bit resolution" digital irradiation sensor could be beneficial in many ultra-low power applications where precise dose measurement is not an issue as long as a certain percentage of error is tolerated. This block theoretically consumes no DC power and the only current draw happens during the transition period. This work can however be expanded to other digital blocks which are capable of more bits of resolution to give a more precise measurement of radiation. Such a device is introduced, designed, laid out, and simulated as part of the future works. 


\subsection{X-ray Power Harvesting Using Diodes}

The novel energy harvesting mechanism presented in this section utilizes photodiodes to harvest X-ray energy to potentially power the circuit blocks on the radiation measurement chip. Two types of photodiode devices were considered: 1) discrete (purchased) 2) fabricated on-chip. Due to initially more promising results from the discrete diodes, the majority of experiments focused on this device and less concentration on case 2 .

It is intended to investigate whether diodes could be used to harvest radiation energy and turn it into useful energy to power circuitry under radiation. Diodes have traditionally been used as radiation detectors. Under radiation, when diodes are reverse biased, the width of the depletion region increases and an electric field is built up across it. The ionizing radiation generates electron-hole pairs in the depletion region and the electric field within that region separates the charges before they could recombine. This collected charge is then converted to a voltage signal through the use of a transimpedance amplifier. Diodes, unlike MOSFETs do not measure cumulative dose or in other words they do not have a "memory"; hence, they need to be sensed constantly and data be collected continuously. This experiment aims to use the diodes as photovoltaic devices under ionizing radiation.

Silicon as a semi-conductor is used in a range of visible light wavelengths to generate current and hence useful power. However, using it for the same application under ionizing radiation does not seem possible since higher energy photons beyond the absorption of silicon will pass through without any interactions.

Experiments were performed on discrete $\mathrm{p}-\mathrm{i}-\mathrm{n}$ diodes manufactured by 
Luna Optoelectronics as shown in Figure 4.13 [91]. Figure 4.14 shows the responsivity of the diodes. It can be seen that longer wavelengths have a better responsivity than the shorter wavelengths. Silicon p-i-n diodes either would not interact with ionizing radiation or if they do, recombination rates would be high in an unbiased mode to generate a significant current; therefore, three different scintillating material were chosen and used to compare against the case without any scintillating material on it.

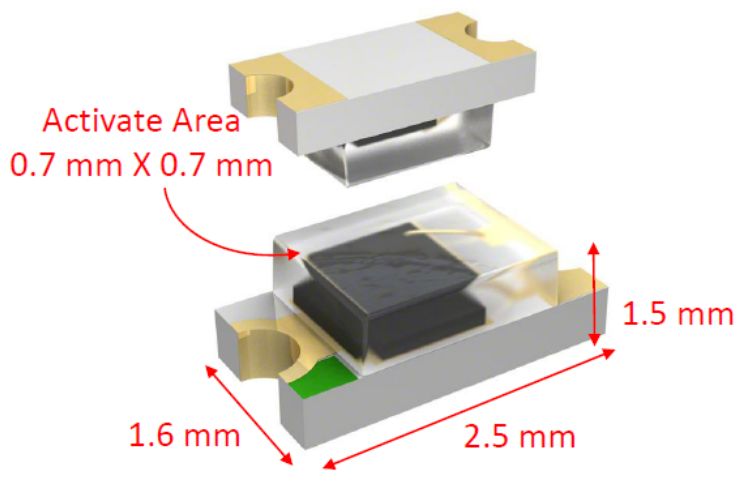

Figure 4.13: Discrete p-i-n diode dimensions [91]

As the integrated chip technology has found its way into ultra low power wireless sensor applications, using photodiodes as energy-harvesting devices has gained serious attraction and popularity. The energy source focused on in a large portion of the photodiode energy harvesting devices reported in numerous publications is the visible spectrum of light and to a lesser extent mechanical, thermal, or chemical sources. [92-94]. A more extensive review of energy harvesting techniques is presented in [95]. The main focus of these publications is to further increase the photo-efficiency of the on-chip integrated junction diodes by manipulating the different variables available through the commercial or non-commercial fabrication technologies for junction diode 


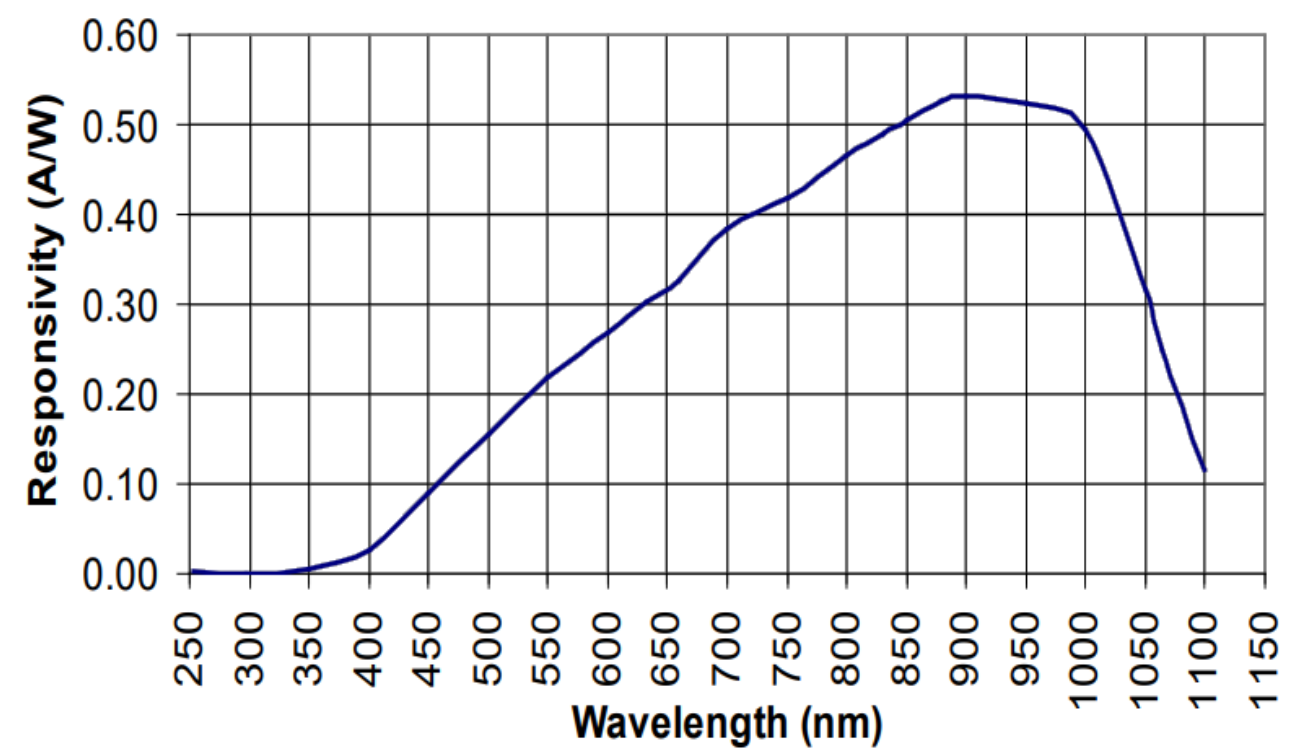

Figure 4.14: Discrete diode spectral response across the spectrum [91]

designs. Different junction areas, vertical and horizontal structures, series or parallel combinations of the devices are some of the reported areas that have been worked on.

\subsubsection{Photodiode theory}

Many publications have discussed photovoltaic effects in detail such as in $[84,96,97]$. A brief theoretical background is provided to set the grounds for the following section. The basic operation of a photodiode in photovoltaic mode are as follows:

1. The generation of light-generated carriers;

2. The collection of the light-generated carriers to generate a current;

3. The generation of a large voltage across the device;

4. The dissipation of power in a load or parasitic resistances;

A silicon photodiode has a single bandgap $E_{G}$. A photon of light with less energy than the bandgap is not absorbed and passes through the device without any interactions, 
but when the incident photon energy is greater than $E_{G}$, the photon is absorbed by an electron in the valence band which raises to the higher energy conduction band. Photon energy and wavelength have an inverse relationship as follows:

$$
\lambda=\frac{c}{v}=\frac{1.24}{h v}
$$

where $\mathrm{c}$ is the speed of light, $\lambda$ is the wavelength in micrometers, $h$ is Planck's constant and $\nu$ is the photon's frequency.

The carriers are generated everywhere throughout the volume of the photodiode; electrons in the p-type doped and holes in the n-type doped parts are considered minority carriers which means that there is a probability of them recombining and hence not contributing to the generated power. They can only exist for a length of time equal to the minority carrier lifetime. But a portion of the generated carriers that reach the depletion region of the diode get swept across the junction and if there is an external load connected across the photodiode, they contribute to the light-generated current. The electron then dissipates its energy through the external load. The reason for this current is the built-in electric field across the diode junction which separates the electrons and holes generated. In order to have useful power, a light-generated current and voltage (photovoltaic effect) must be present. Under short circuit conditions, there is no build up of charge across the junction, hence all the light-generated carriers exit the device. However, if there is no path for this current to flow, the collection of the generated carriers causes an increase in the ptype carriers in the p-side and the n-type carriers in the n-side. This separation of charge creates an electric field at the junction which is in opposition to that already across the junction. A reduction in the net electric field increases the forward bias diffusion current. The light generated current is balanced out by the forward bias 
diffusion current and a net zero current is reached. The voltage required to create this balance is the open circuit voltage $V_{o c}$.

Equation 4.2 describes the I-V characteristic of a photodiode under illumination:

$$
I=I_{s}\left(\exp \left(\frac{q V}{n k T}-1\right)\right)-I_{L}
$$

where, $I_{L}$ is the light-generated current, $I_{s}$ is the saturation current, $V$ is the voltage across the diode, $k$ is the Boltzmann constant, $T$ is the absolute temperature of the $\mathrm{p}-\mathrm{n}$ junction, and $q$ is the magnitude of charge of an electron. Open circuit voltage is found by setting the net current to zero as shown in Equation 4.3:

$$
V_{o c}=\frac{k T}{q} \ln \left(\frac{I_{L}}{I_{s}}+1\right) \approx \frac{k T}{q} \ln \left(\frac{I_{L}}{I_{s}}\right)
$$

Figure 4.15 illustrates the photodiode in the photovoltaic mode of operation. The voltage and current at which the maximum output power is generated is shown in Figure 4.16.
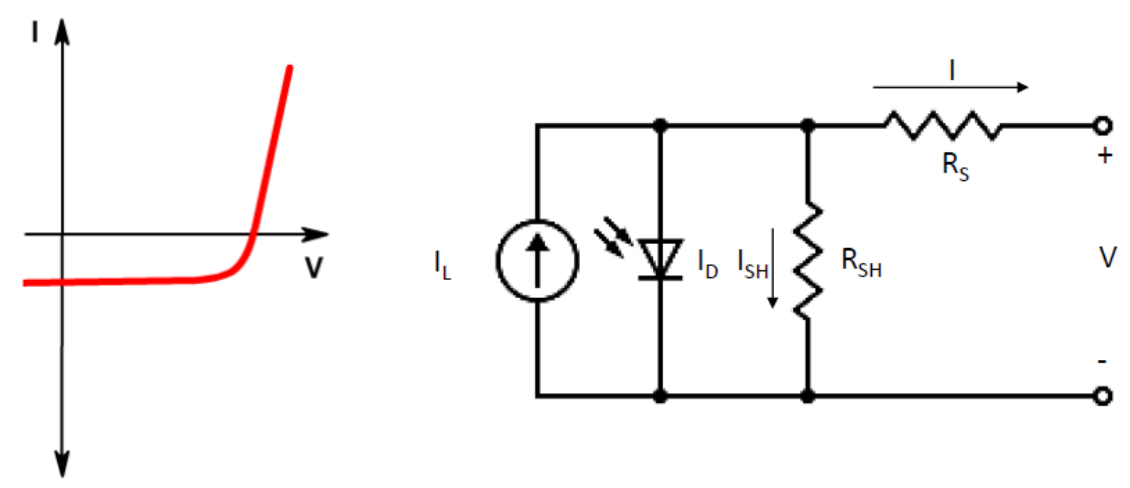

Figure 4.15: Photovoltaic operation of a diode

The absorption coefficient shown in Figure 4.17 determines how far the photon of light 


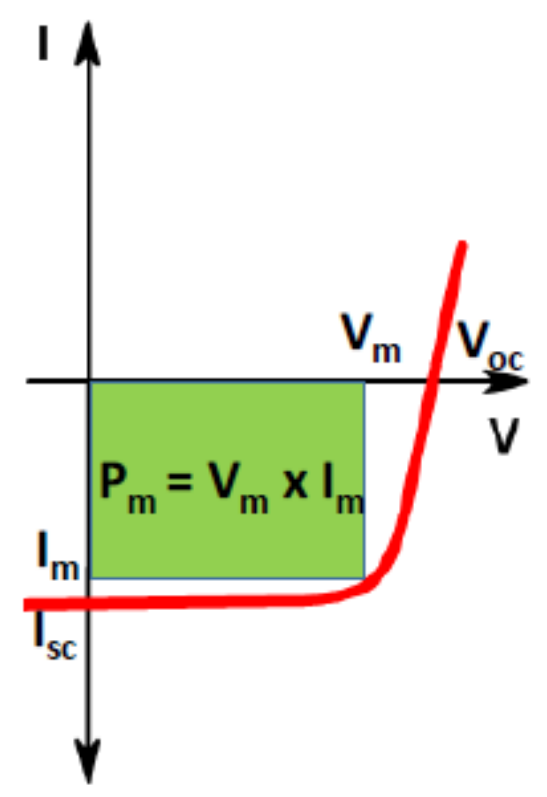

Figure 4.16: $\mathrm{Pmax}$

of a certain energy would travel before being absorbed. It depends on the material and also the wavelength of light. The inverse of the absorption coefficient is the absorption depth shown in Figure 4.18, which describes how deeply light penetrates before being absorbed. Higher energy light has a shorter wavelength and shorter absorption depth [98].

Considering that $\mathrm{X}$-ray photons generated in the blood irradiator and the $110 \mathrm{kVp}$ source are of much higher energies and cover a wide spectrum of photon energies, silicon alone has a relatively low absorption coefficient compared to visible light spectrum. This means that even though carrier generation occurs within the silicon body, either the interactions are less probable or the carriers generated recombine before crossing the depletion width hence insignificant contribution occurs shown in Figure $4.19[99]$. 


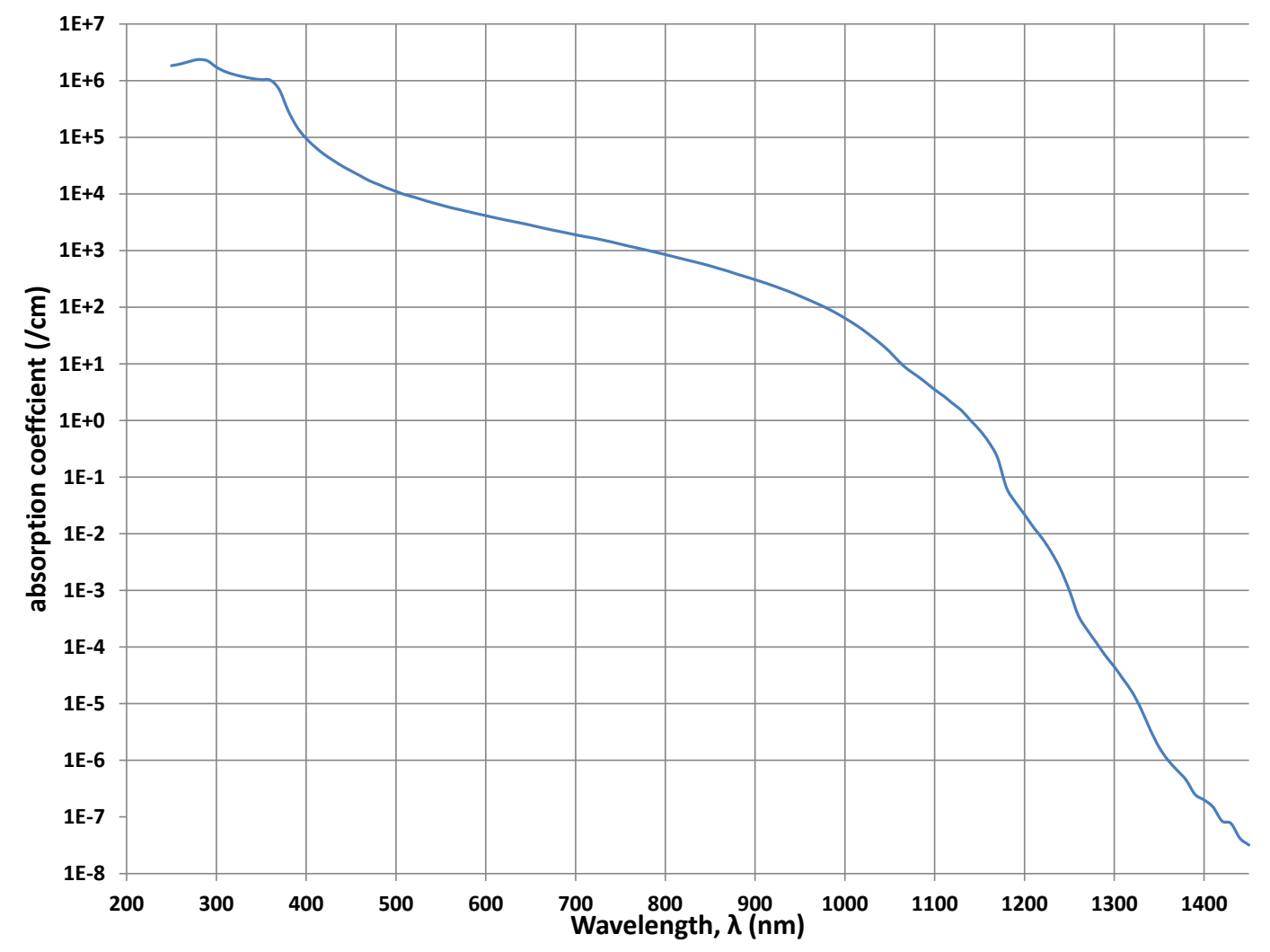

Figure 4.17: Silicon absorption coefficient at 300k [98]

Knowing the shortcoming of silicon photodiodes in high energy photon environments, a new approach was employed in generating power under radiation using these diodes. Scintillating material were employed to convert X-ray photons to visible spectrum photons that can be detected and absorbed by the photodiodes under radiation. A scintillating material is such that when excited by ionizing radiation, it absorbs its energy and re-emits the energy in the form of light. Numerous publications have described in detail the mechanisms of scintillation in matter and their applications $[100,101]$. Below, an overall introduction of scintillating material is presented.

Scintillation is a relatively complex process which can be described in three general steps:

1. Conversion, 


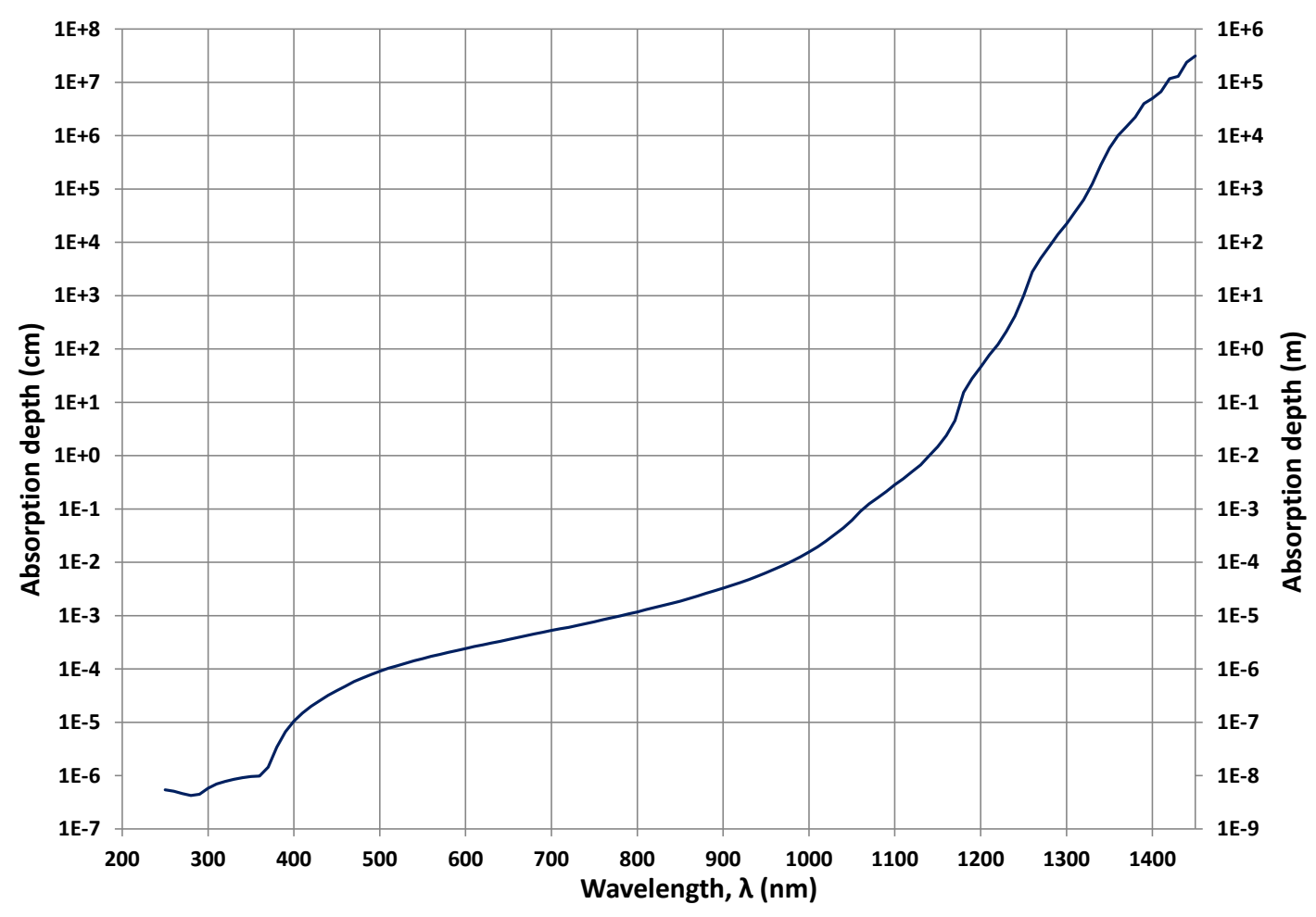

Figure 4.18: Silicon absorption depth at 300k [98]

\section{Transport,}

3. luminescence.

Through the conversion process, a multilevel interaction of a high energy photon with the lattice of the scintillating material occurs through the photoelectric effect and Compton scattering effect. Photon energies below $100 \mathrm{keV}$ interact predominantly through photoelectric effect. Through this step, many electron-hole pairs are generated [102]. During the transport stage, electrons and holes travel through the material during which repeated trapping at defects may occur and energy losses are possible. This stage takes considerable time because of the repeated recapturing of the carriers. The final step is the luminescence which is the consecutive trapping of the charge carriers at the luminescence centers and their radiative recombination. 


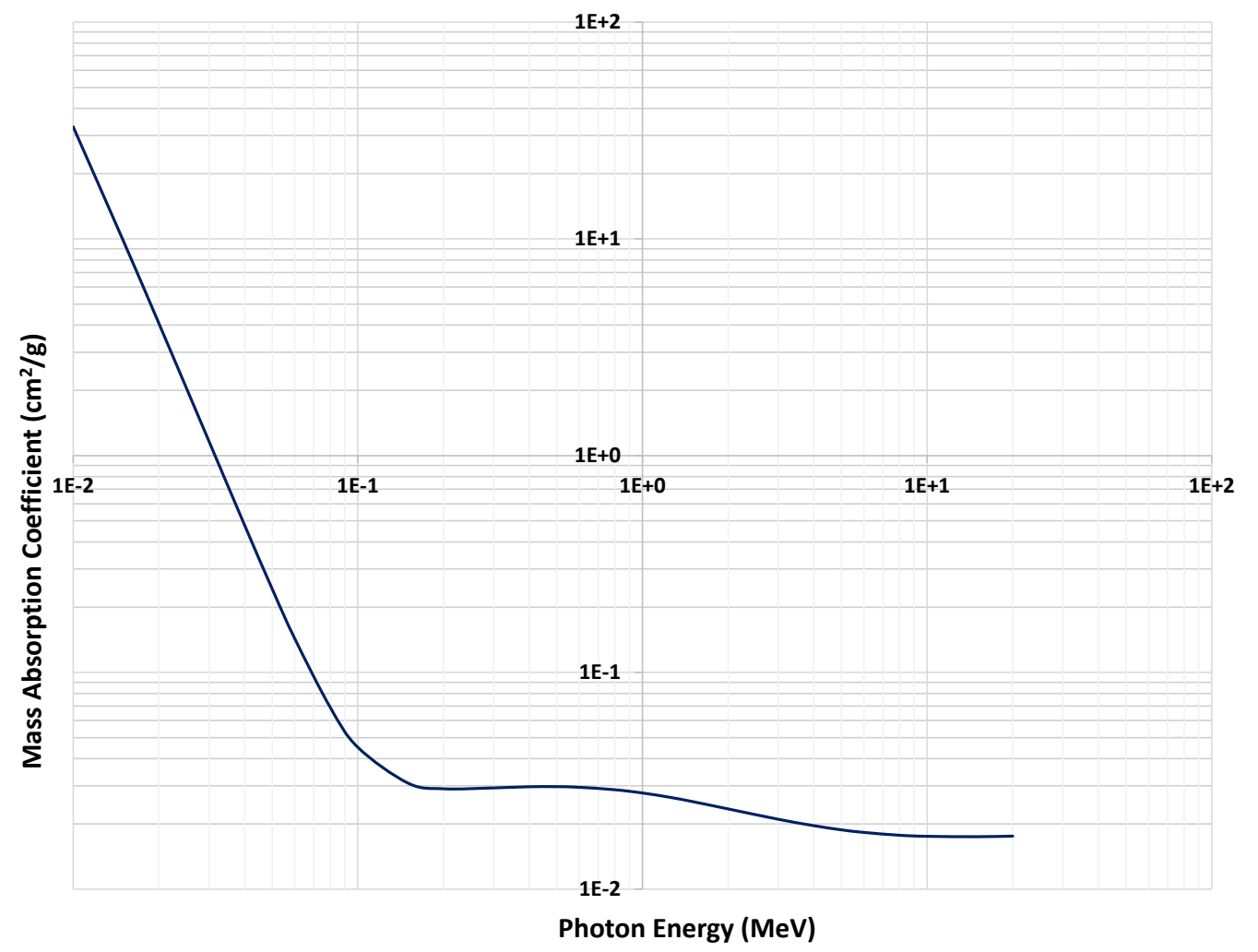

Figure 4.19: Silicon mass energy abosrption coefficient [99]

The radiative recombination is the stage at which the light generation occurs. A good scintillating material must be composed of high atomic number and high density elements in order to absorb the X-ray radiation. It must also produce a large number of visible light photons for each absorbed X-ray photon. Scintillating materials are generally used to detect X-rays when combined with electronics. Photodetectors (photodiodes) are coupled with the scintillating materials to capture the visible light photons generated as per X-ray radiation which in turn generate an electrical signal which can be analyzed. Figure 4.20 shows the simplified phenomena.

There are many scintillator characteristics such as light yield, X-ray stopping power, decay time, chemical stability and radiation resistance, and linearity of light response with the incident X-ray photon energy. The more important of these characters in the 


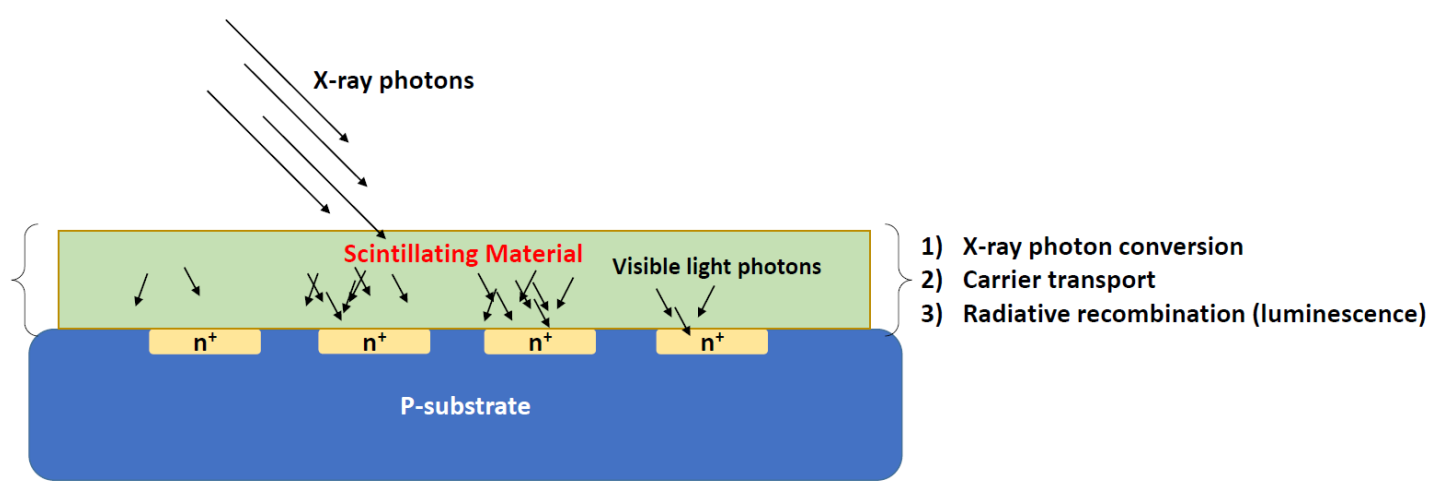

Figure 4.20: Scintillation of X-rays and consequent photo detection and conversion to electrical signal

case of utilizing scintillators for power generation and not for detection are the light yield and the X-ray stopping power. Light yield is the measure of visible photons generated per X-ray photon measured in $\frac{1}{M e V}$. Equation 4.4 [103] demonstrates the dependence of light yield on the following variable.

$$
N_{p h}=\frac{E}{\beta E_{g}} S Q
$$

$E$ is the incoming photon energy, $\beta$ is a number between 2 and $3, S, Q$ are quantum efficiencies of the transport and luminescence stages.

The X-ray stopping power or attenuation coefficient depends on the thickness of the scintillator, the density $\rho$ and the effective atomic number $Z_{\text {eff }}$.

For the experiment, three scintillating materials were chosen:

1. $\mathrm{Y}_{2} \mathrm{O}_{3} / \mathrm{Eu}$ : Red luminescence; Yttrium Oxide, europium doped which fluoresces at $609 \mathrm{~nm} \rightarrow 613 \mathrm{~nm}$ (package \#3); [104]. According to [105] and [106], Europium doped Yttrium Oxide is widely used for radiation dosimetry applications among others. 
2. $\mathrm{CeMgAl} \mathrm{M}_{11} \mathrm{O}_{19} / \mathrm{Tb}$ : Green luminescence; Cerium Magnesium Aluminate, Terbium Doped which fluoresces at $544 \mathrm{~nm} \pm 2 \mathrm{~nm}$ (package \#1) [107].

3. $\mathrm{BaMgAl}_{10} \mathrm{O}_{17} / \mathrm{Eu}$ : Blue luminescence; Barium Magnesium Aluminate, Europium doped which fluoresces at $450 \mathrm{~nm} \pm 10 \mathrm{~nm}$ (package \#2) [108]. According to [109], Europium doped Barium Magnesium Aluminate is an important blue emitting phosphor.

Without further investigation into the chemical compositions of the above-mentioned scintillators, they were applied to identical discrete diodes shown in Figure 4.21. The diodes were verified under visible light source to ensure their proper operation and close response prior to being subjected to the materials. The thickness of the scintillating materials are the same and about $1 \mathrm{~mm}$ thick. Packages \#5 \& 6 demonstrate insignificant difference in photovoltaic performance under visible light illumination since package \#5 is only covered with transparent fill and package \#6 is left exposed. Package \#6 is used as the reference device to compare against the other three packaged devices under X-ray radiation.

First experiment involves exposing the packages to the $110 \mathrm{kVp}$ X-ray source. The photon energy spectrum is not exactly known for this machine. The aim of this experiment is to verify that diodes under radiation can be used not as detectors and dosimeters, but rather for power generation to potentially be used to power the dosimeter chip under exposure. Figure 4.22 shows the results of the experiment.

As seen in Figure 4.22, the diode package with no scintillating material coverage generates three times the highest current produced by the scintillation material covered diode under $110 \mathrm{kVp}$ X-ray. This could be due to the low photon energies out of the X-ray source and also low photon flux. Diode without the cover has a short circuit 


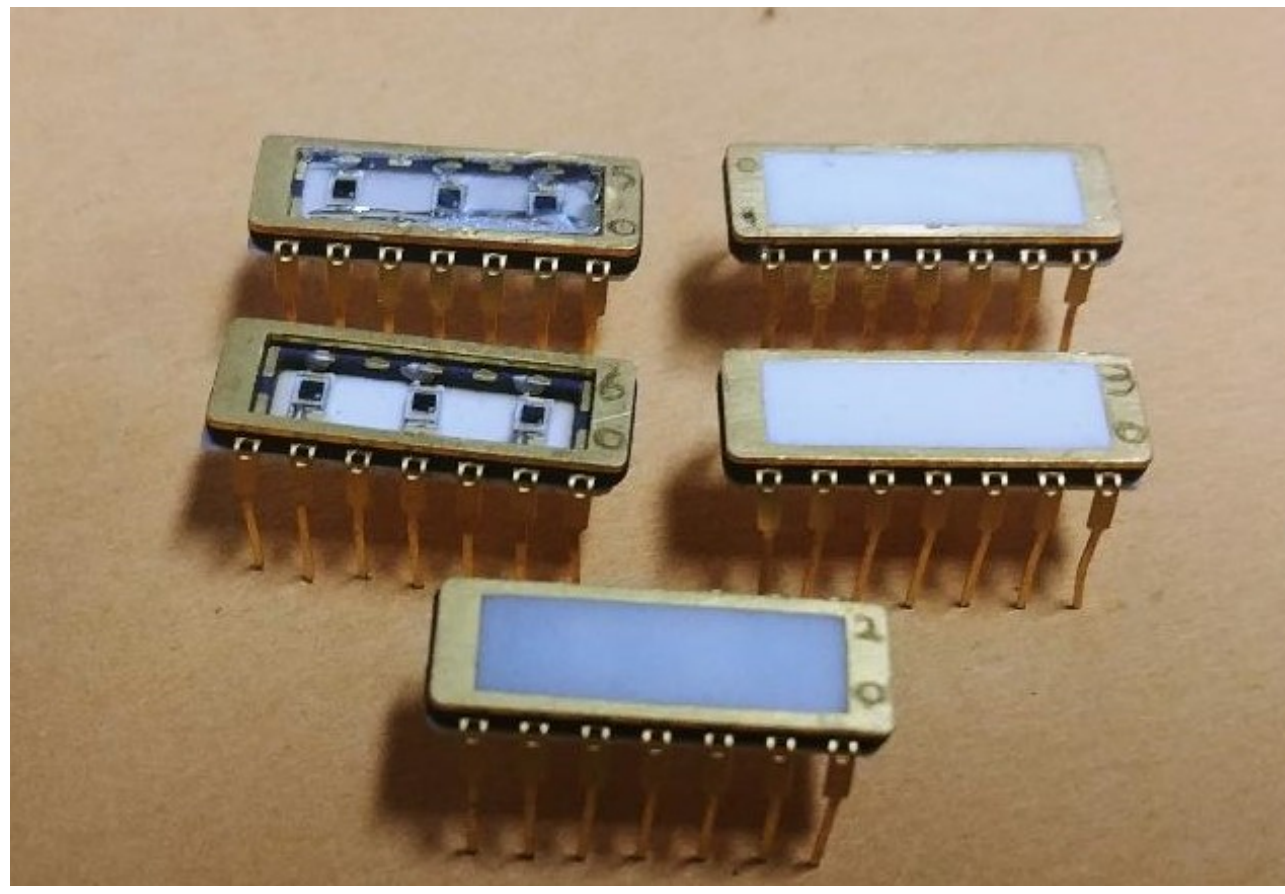

Figure 4.21: Packaged discrete diodes with or without scintillating material

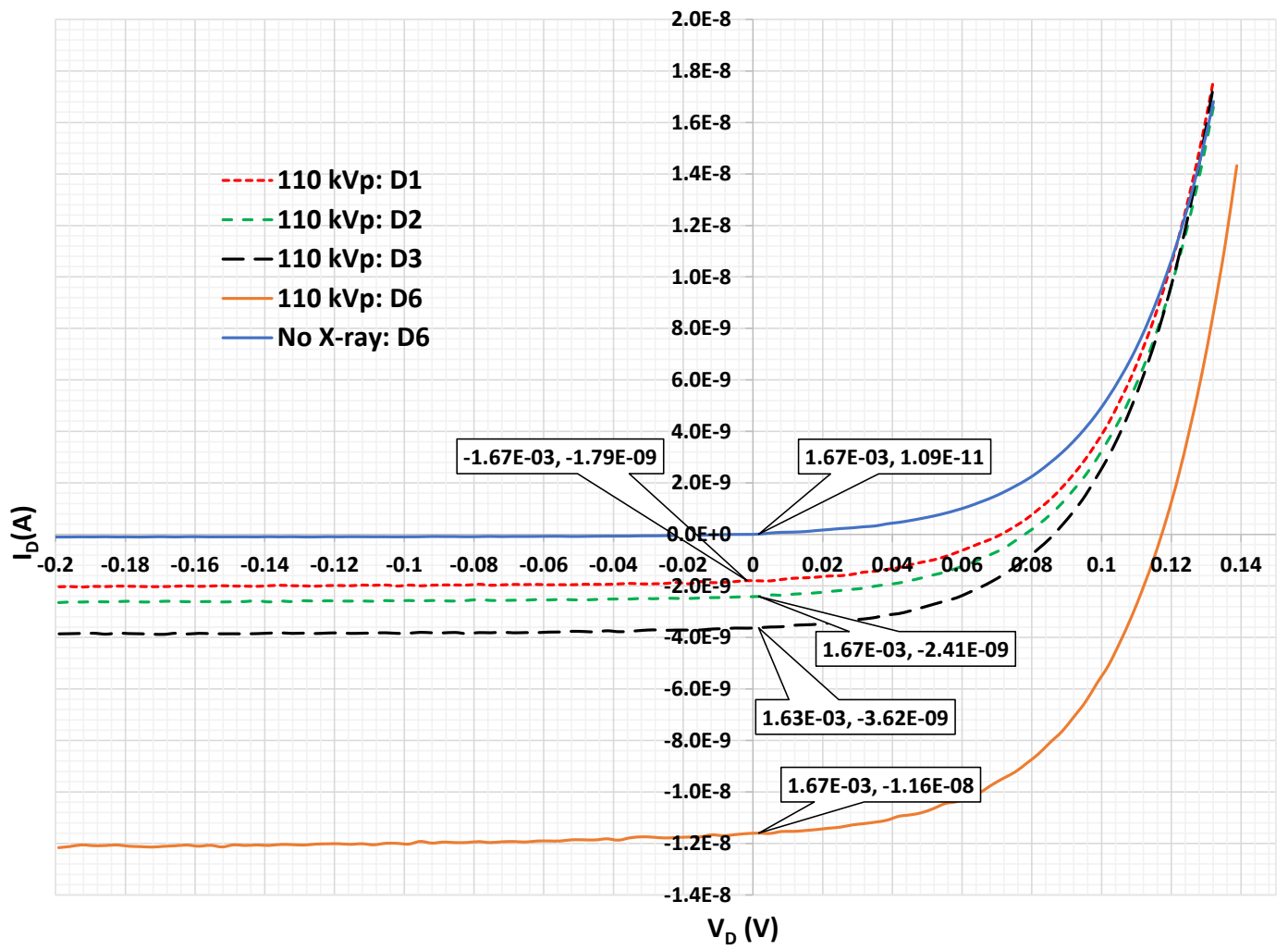

Figure 4.22: Discrete diode packages 1,2,3, and 6 under $110 \mathrm{kVp}$ X-ray source irradiation 
current $I_{S C}=11.6 \mathrm{nA}$ and open circuit voltage $V_{O C}=118 \mathrm{mV}$. The generated maximum power for D6 is shown in Figure 4.23 and it is at $0.7 \mathrm{nW}$. A negative power is taken as light-generated power.

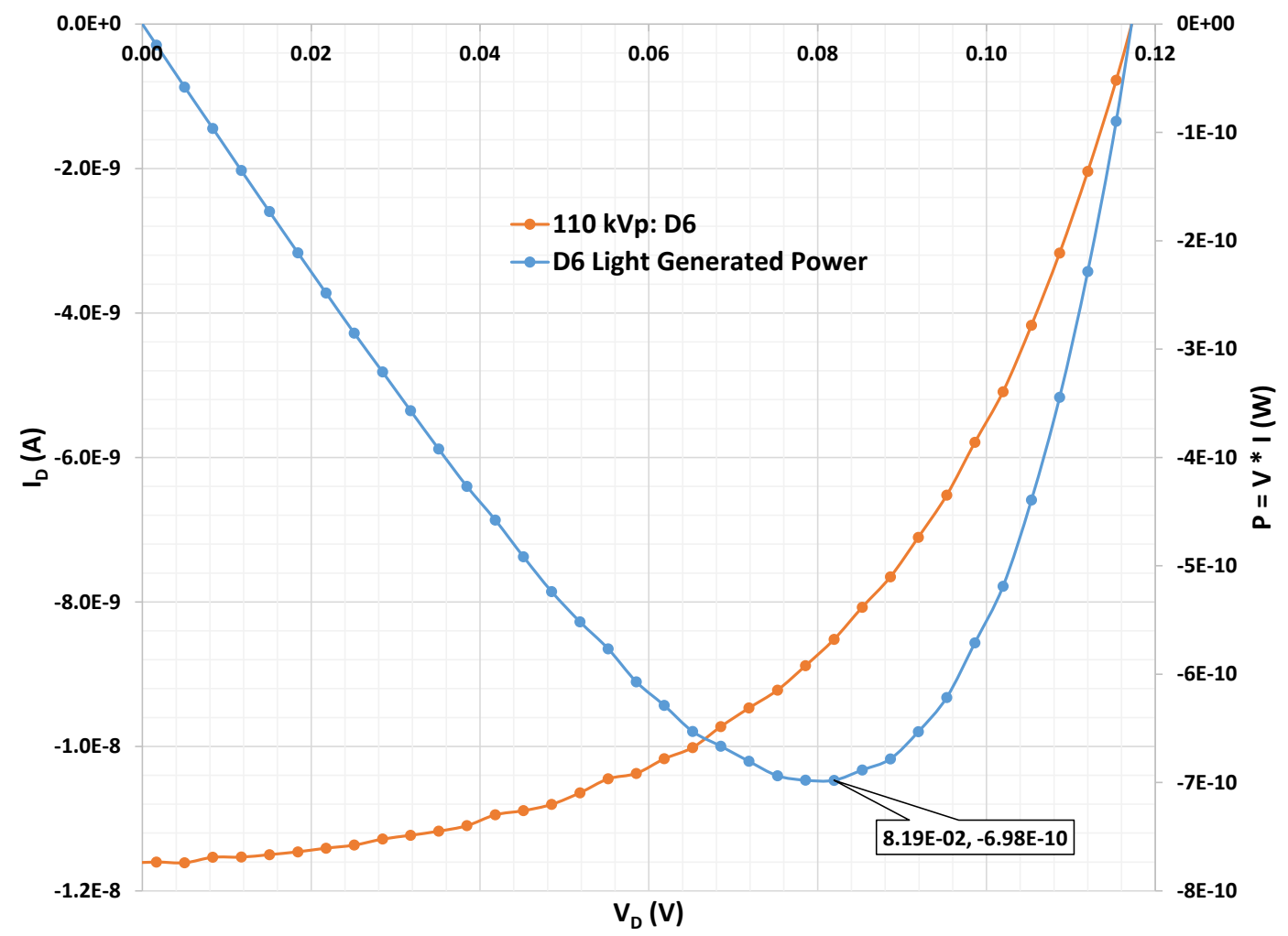

Figure 4.23: Generated power under $110 \mathrm{kVp}$ curve of D6 package

Higher photon energy and higher photon flux experiment using the blood irradiator machine was performed on the same diode packages and the results are shown in Figure 4.24.

As the experiments illustrate, package $\mathrm{D} \# 3$ which is coated with $\mathrm{Y}_{2} \mathrm{O}_{3} / E u$ demonstrates the most short circuit current $I_{S C}=118 \mathrm{nA}$ to $146 \mathrm{nA}$. The 3 diodes in package $\mathrm{D} \# 3$ illustrate a spread in results which could be caused by density or thickness variations in the scintillating material. This variation is not seen in the other 


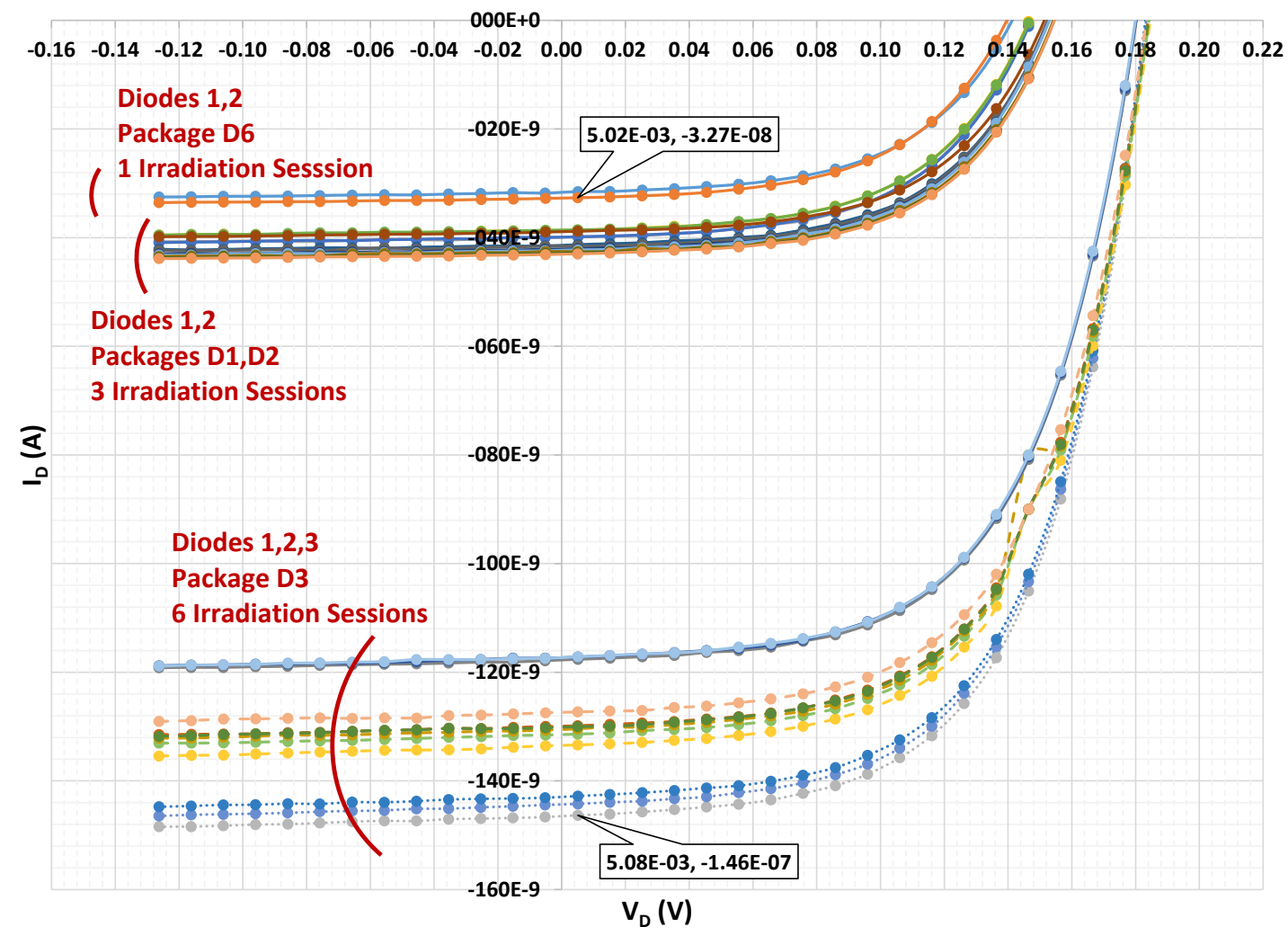

Figure 4.24: Diode packages D1, D2, D3, and D6 irradiation using the blood irradiator machine

cases mainly due to the fact that the other cases have lower currents to start with, so a percentage variation in their diode-to-diode performance is insignificant.

Package D\#6 has a short circuit current of $I_{\mathrm{SC}}=33 \mathrm{nA}$ compared to the $110 \mathrm{kVp}$ case which outputs double the current. It is believed that higher energy photons in this case have more interactions with the photodiode which help generate more electronhole pairs than in the previous case and also package $\mathrm{D} \# 3$ emits in the red spectrum of light which travel further within the thickness of the scintillation material which could be the reason for the higher photon conversion efficiency.

Lastly, the series and parallel combinations of 3 diodes in each package were created and irradiated by the blood irradiator machine as shown in Figure 4.25. As seen in the 
figure, package D\#3 is dominant in current and voltage generation under irradiation. Therefore, either of the parallel or the series combinations of this diode can be used to power ultra low power circuits.

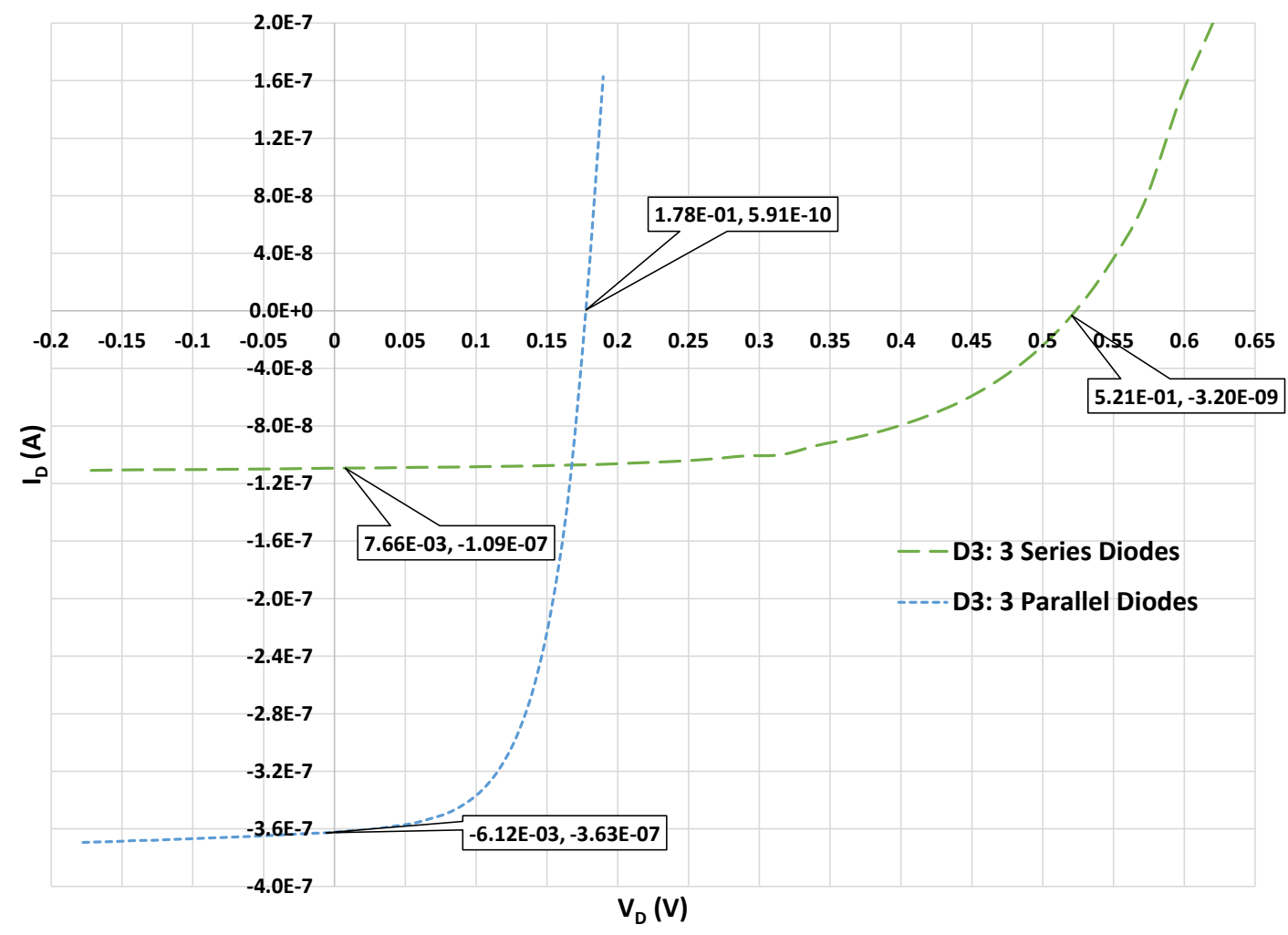

Figure 4.25: I-V characterisitics of series and parallel combinations of 3 diodes in D3 package

Figure 4.26 shows the light generated power in a series and parallel combinations of three diodes in package $\mathrm{D} \# 3$.

Future investigations on selecting more effective scintillating material is required. Using a diodes coated with $\mathrm{Y}_{2} \mathrm{O}_{3} / \mathrm{Eu}$ proved to be effective in generating voltages and currents that are capable of driving low power low voltage digital circuits such as the FG-inverter introduced previously. 


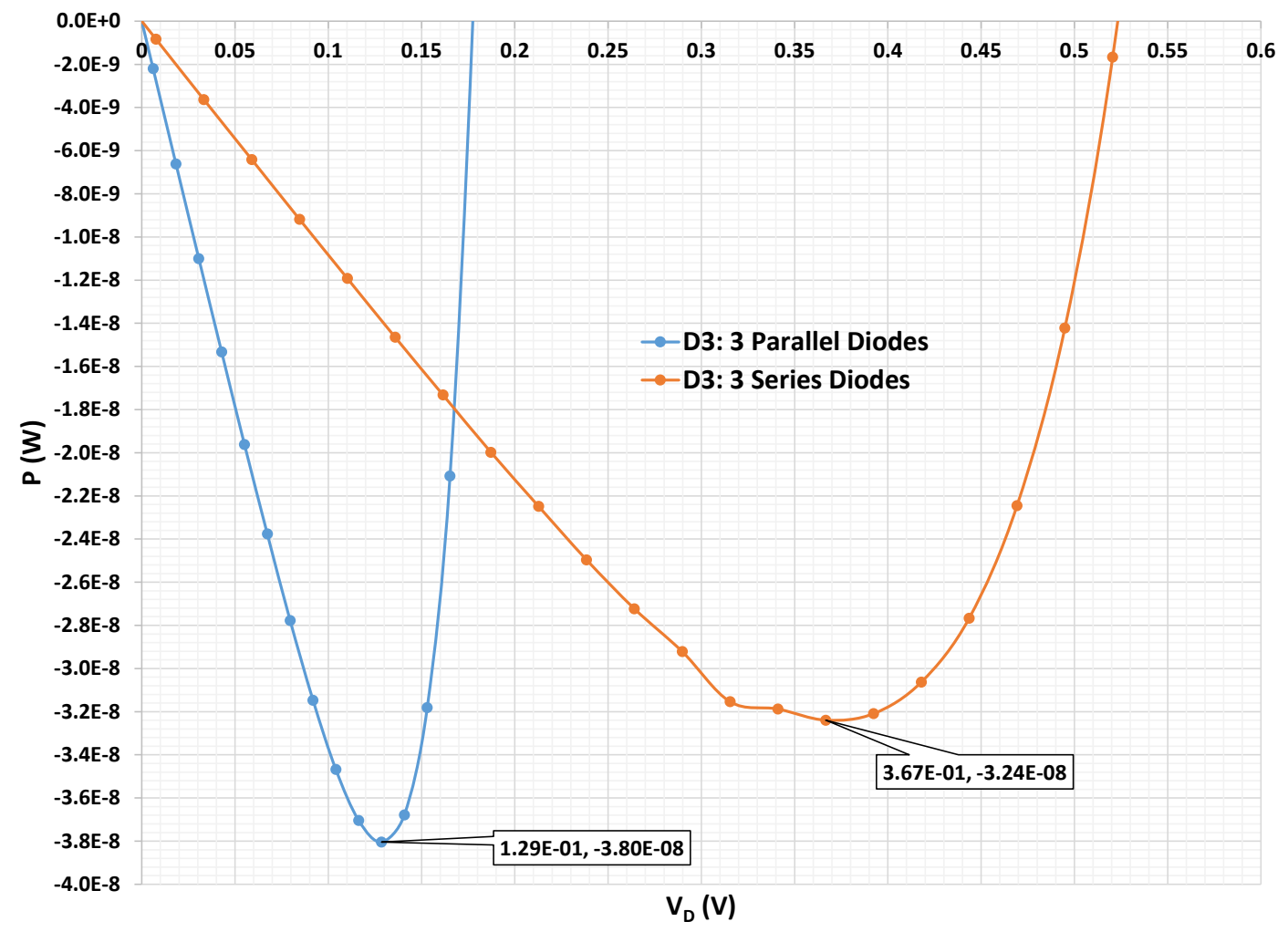

Figure 4.26: Power generated in a series and parallel combination of D3 diodes under blood irradiator radiation

\subsection{Chapter Conclusion}

This chapter presents methods to improve the efficiency of the MOSFET radiation sensors by reducing the active power consumption of the design. For this purpose, new design techniques based on NMOS and CMOS topologies are investigated. The following conclusions can be drawn from this chapter:

1. At first, FG-NMOS irradiation sensors are studied to validate their utilization in the desired application. Using a $110 \mathrm{kVp}$ and the blood irradiator X-ray sources it is observed that the precharged sensor can be completely discharged after 6 mins. The results thus provide evidence for their use as a radiation dosimeter.

2. Using the initial results on an FG-NMOS sensor, an inverter design is proposed 
that can work as a 1-bit resolution substitute to the PMOS sensors presented in Chapter 4. This is the first time that floating gate technology is used in an inverter configuration to measure the intensity of radiation exposure. The design is profoundly better in terms of power consumption as compared to the PMOS sensor design. The topology thus presented shows a resolution of 1 bit with a total power consumption of $\approx 600 \mathrm{nW}$ during the transition period. This represents a reduction of $98 \%$ in the power consumption compared to the FG-PMOS case.

3. To further cater to the need for a power source, energy harvesting techniques have been studied using off-the-shelf discrete photodiodes. The diodes are coated with three different scintillating materials to study their effects on the I$\mathrm{V}$ characteristics. When exposed to $\mathrm{X}$-rays, $\mathrm{Y}_{2} \mathrm{O}_{3}$ based designs demonstrated the highest short circuit current. A maximum current of $146 \mathrm{nA}$ is induced through exposure to radiation. This solution shows the merit of integrating photodiodes with the radiation sensors as a secondary source of energy whereby these devices are charged by the radiation themselves.

4. The concept of a photodiode is implemented using discrete components; however, similar results could be obtained from on-chip designs. As a future research, integration of these photodiodes on the same chip as the FG-MOS sensor can be studied to reduce the sensor's dependence on an external power source. The eventual goal will be to make these sensors battery-less. 


\section{Chapter 5}

\section{Non-Volatile Memory Design}

\section{$5.1 \quad$ Introduction}

In this chapter, the third thesis objective is presented: to design an ultra-low power, variable supply, non-charge based, permanent memory cell that stores an identification bit string on chip for each dosimeter tag. This is crucial to the operation of the RFID dosimeter tag in two ways. First, it is necessary to be able to identify a blood bag from another. There may be multiple blood bags close to each other with each having a tag affixed on it. We require identifying each blood bag from others for tracking purposes to avoid tampering with the bags. Second, it is necessary to be able to initiate a command to a specific tag to send the radiation data back to the reader. If there is no specific identification of the chips, once a read data command is sent to the tags, all of them would respond back at the same time causing jamming or possible error in reading back the dosimetry data.

The challenges faced in designing a robust memory cell are as follows:

1. Ensuring low power operation and operation from a range of supply voltages $(\sim 0.5 \mathrm{~V}-1.2 \mathrm{~V})$ of the memory (ID) block, since it will be powered by wireless RF 
illumination or a super capacitor as a secondary power source. The emphasis is on the read operation in this case as the write operation will have been performed once by either the chip manufacturer or the tag supplier.

2. Ensuring the correct operation of the memory, i.e. presenting the same bit stream, without missing a bit or an incorrect bit read. Clock spread and comparator layout symmetry are important considerations.

Accordingly, the specific thesis sub-objectives are as follows:

5.1. Investigate and select the best memory type to represent each bit and the best architecture for this application;

5.2. Investigate and design ultra-low power write and read operation circuitry for the selected memory architecture mentioned above;

5.3. Validate correct operation of the memory block in terms of robustness to variable supply voltage and insensitivity to ionizing radiation.

Considering the different types of one-time programmable (OTP) memory presented in the literature review, we propose the eFuse memory cell as the ultimate candidate for implementing the chip ID in this thesis for the following reasons:

1. eFuse cells are readily available in the $0.13 \mu \mathrm{m}$ RF-CMOS technology used for the low power X-ray radiation sensor tag system implementation.

2. eFuse does not require higher than $3.3 \mathrm{~V}$ limit of the thick gate oxide IO transistors for programming as opposed to the antifuse option.

3. Antifuse cells would only have been an option if their behavior (programming and reading characteristics) were known through other publications. If this thesis were to choose antifuses, a series of experimental verifications would be 
required to determine the optimum size and voltages for programming. Also, layout would have become an important factor.

For the readout (sensing) block, the shared reference topology is proposed in order to increase the sensing robustness while reducing circuit complexity and area. Also having one reference means less current consumption which ultimately means less power consumption.

As for the number of bits, it was decided that an 8-bit memory ID is an adequate size. A greater number of bits was not an option due to shortage of time for design; however, it is possible to expand the number beyond 8 bits if need be in the future.

\subsection{Novel non-Volatile Memory Design and Imple- mentation}

While antifuse memory cell has been widely adopted as a solution to NVM design, in this thesis work, the most important parameter is the reliability and the correct operation. Due to tight schedule for design and fabrication, and also due to variation of opinions with regards to design decisions (i.e. applied programming voltage, MOSCAP size, access transistor size, etc.) among researchers, this design architecture is not chosen for the purpose of this thesis work. The main advantage noted by authors in utilizing the antifuse as a memory solution is its smaller footprint and programming current compared to efuse memory structure. However, smaller area and programming current are not of considerable concern in this work. Also, eFuse links are readily provided within the technology package used in this thesis; therefore, eFuse memory cell is chosen to create the ID cell.

In this research work, an original NVM design was undertaken in $0.13 \mu \mathrm{m}$ RF-CMOS 
to meet the unique wireless dosimeter specifications. An 8-bit non-charge based nonvolatile one-time programmable read-only memory (OTPROM) using the electronic fuse (e-Fuse) offered in the standard $0.13 \mu \mathrm{m}$ RF-CMOS with no additional processing overhead mask is proposed in this research. The sensing circuitry is designed to operate stably in the supply range of $0.53 \mathrm{~V} \rightarrow 1.2 \mathrm{~V}$. This enables the block to operate correctly even if the supply voltage drops due to unforeseen circumstances or operating off of a super capacitor. Low voltage operation is possible through a supply independent current reference block setting the current through the reference and each eFuse. Another significance of this design is that it overcomes the challenge of isolating the programming circuit block - which operates at $3.3 \mathrm{~V}$ - from the sensing circuit block which operates at low voltages using $1.2 \mathrm{~V}$ MOSFETs that cannot withstand higher supplies. The lowest sensing current of $\sim 114 \mu \mathrm{A}$ is drawn at a supply of $0.53 \mathrm{~V}$, resulting in a power consumption of $60.42 \mu \mathrm{W}$. The overall area of the memory block is $220 \mu \mathrm{m} \times 140 \mu \mathrm{m}$. The sensing block utilizes the strong arm latch design which only requires a clock signal to precharge the line and instead of passing through each eFuse like in other designs, it passes through the input transistors which avoids extra metal routing.

To the best of the author's knowledge, there is no eFuse memory design done in this RF-CMOS technology having the design innovations mentioned above.

An "un-burnt" e-Fuse measures $\sim 50 \Omega$ to $150 \Omega$ and a burnt one measures $\sim 4000 \Omega$. Therefore, an E-fuse memory cell requires a write circuitry that burns each eFuse by applying a high-voltage $(\sim 3.3 \mathrm{~V})$ and a large current $(\sim 10-15 \mathrm{~mA})$ for programming and also a low power sense circuitry that reads the bit string produced by the combination of burnt and unburnt e-Fuses. The application of $3.3 \mathrm{~V}$ to each cell requires the use of high-voltage $(3.3 \mathrm{~V})$ variation of transistors for the write stage since the 
standard ones cannot withstand such high voltages.

The overall layout image is presented in Figures 5.1 and 5.2. The overall chip measures at $1.5 \mathrm{~mm} \times 1.5 \mathrm{~mm}$ including the bonding pads.

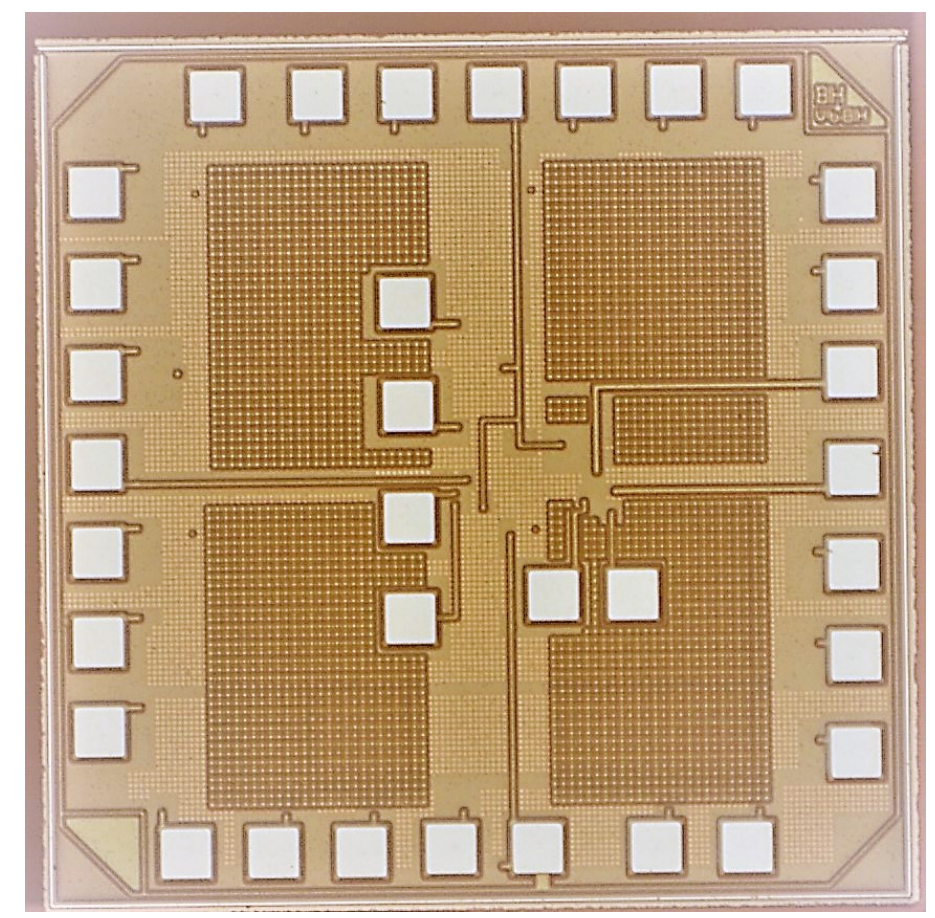

Figure 5.1: A microscopic image of the overall ID chip. The total chip meaures at $1.5 \mathrm{~mm} \times 1.5 \mathrm{~mm}$.

The overall read/write operations of this block require 8 input signals (pads) and 2 supply pads for $V_{D D}$ and ground. Table 5.1 shows the input signals.

Reset is used to clear any possible bit values from the registers and also is a necessary signal in conjunction with Pulse_in to start the ring counter circuit.

Read_clk signal is used for reading out of the memory bit pattern. This clock signal is to be generated on chip through the use of a ring oscillator or any other clock generator block. The operating voltage of this signal matches the read operation 


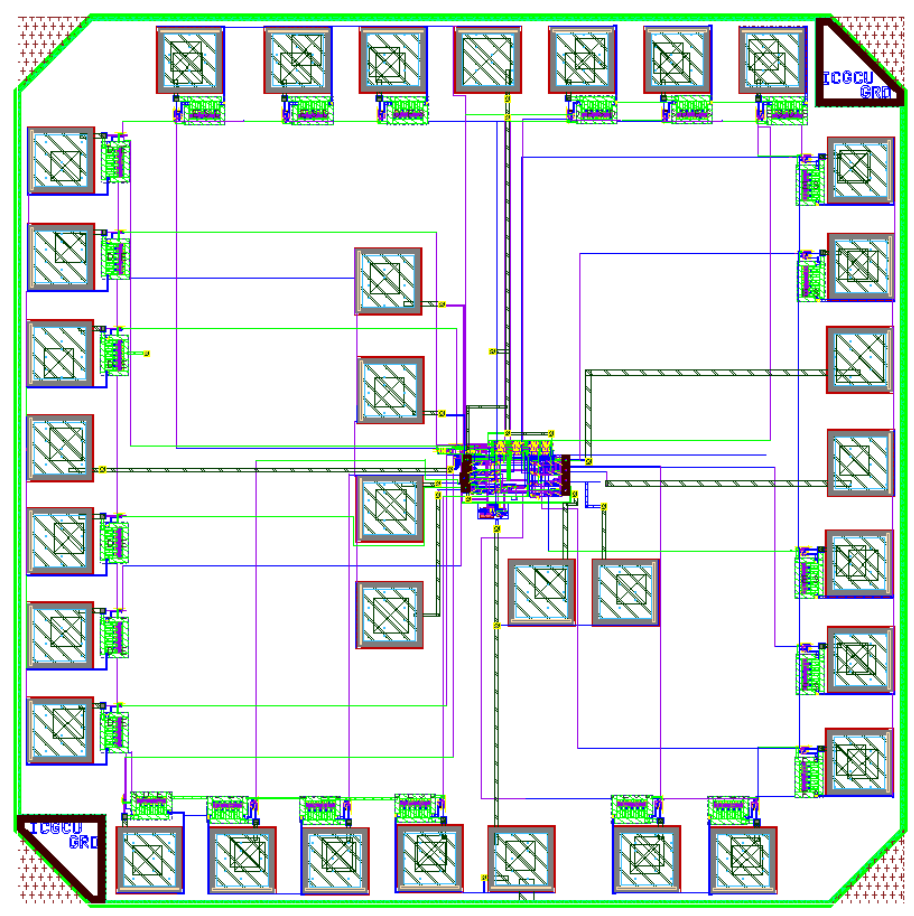

Figure 5.2: Overall memory chip layout including all the input, output and test signal pads

Table 5.1: Input signals for write and read operations of the NVM

\begin{tabular}{|c|c|c|}
\hline Input Signal & Write Operation & Read Operation \\
\hline Reset & $0 \rightarrow 1$ & $0 \rightarrow 1$ \\
\hline Read_clk & $0 \rightarrow 1$ & $01 \times$ many \\
\hline Pulse_in & $0 \rightarrow 1$ & $0 \rightarrow 1$ \\
\hline Write_clk & $0000(01) \times 80000(01) \times 80$ & 0 \\
\hline Data_Write_Enable & $0 \rightarrow 1$ & 0 \\
\hline Data & 0000 bit stream & 0 \\
\hline C_OF & $22 \times 0 \rightarrow 1$ & 0 \\
\hline$V_{\text {DDH }}$ & $3.3 \mathrm{~V}$ & $0.53 \mathrm{~V} \rightarrow 1.2 \mathrm{~V}$ \\
\hline$V_{\text {DD }}$ & $1.2 \mathrm{~V}$ & 0 \\
\hline Gnd & 0 &
\end{tabular}


supply range of $0.53 \mathrm{~V} \rightarrow 1.2 \mathrm{~V}$. From Read_clk through the use of a phase shifter and frequency divider two signals are generated called Comp_clk and $S R_{-}$clk.

Comp_clk is the comparator clock and this signal arrives earlier than the $S R_{-} c l k$. Comp_clk precharges the nodes to $V_{D D}$ on the falling edge as shown in Figure 5.9. On the rising edge of this clock, the evaluation period starts where a decision is made based on the input values to the differential pair.

Pulse_in has the task of starting the ring counter which carries a logical " 1 " through the $8 \mathrm{read} /$ write blocks; the outputs of the ring counter are named RingQi, RQi, RQib.

Write_clk is applied to a separate pad externally to perform the fuse writing task. The clock amplitude is set to $V_{D D}$ of $1.2 \mathrm{~V}$ and not to $3.3 \mathrm{~V}$. This signal is grounded for the reading operation of the block.

Data_Write_Enable is a constant "1" used only during the writing operation. This signal acts on the MUX's used to carry out the write or read tasks.

Data is an 8 bit pattern passed to the registers serially before the writing operation. $C_{-} O F$ enables or disables the access to $V_{\mathrm{DDH}}$ supply. This signal is also necessary for enabling the write operation on each individual fuse.

$V_{D D H}$ is set at $3.3 \mathrm{~V}$ for the writing operation only. This signal also isolates through a PMOS "T2" shown in Figure 5.8 the standard MOSFETs in the reference current source from possible damage. This signal also isolates the input of the comparator VFuse and any possible current path from the Fuse Cell block shown in Figure 5.6 during the writing operation. 


\subsubsection{Programming Circuit Design}

As described briefly above, programming an eFuse within this technology requires a $\sim 3.3 \mathrm{~V}$ supply and $\sim 10-15 \mathrm{~mA}$ of DC current for a duration of $\geq 200 \mu \mathrm{s}[45,46]$. Figure 5.3 shows the first 8 clock cycles that pushes the 8 bit data pattern to the registers before being written in the next 8 clock cycles.

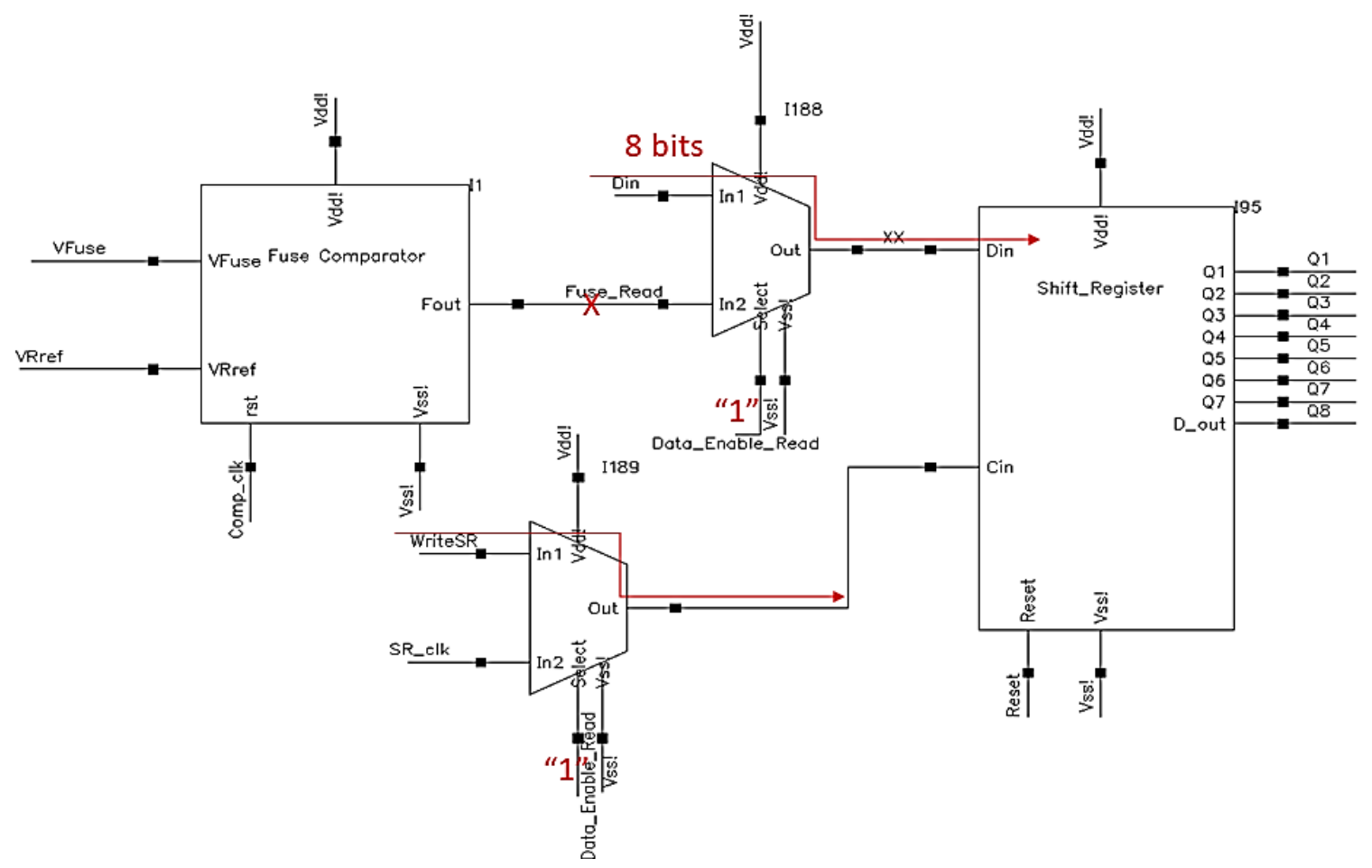

Figure 5.3: Reading in the 8 bit data pattern to the registers to be written to the eFuse

Figure 5.4 demonstrates a more focused picture of the eFuse program/sense circuit block. Figure 5.5 shows the read/write control circuit blocks. To briefly explain the operation of these blocks, the three signals $Q_{i}$, Ring $g_{Q i}$, and $C_{O F}$ need to be "High" in order for the level shifter inside the "Fuse Write Input" block to output a "High $=3.3 \mathrm{~V}$ " signal to enable the programming NMOS. Then the programming current 


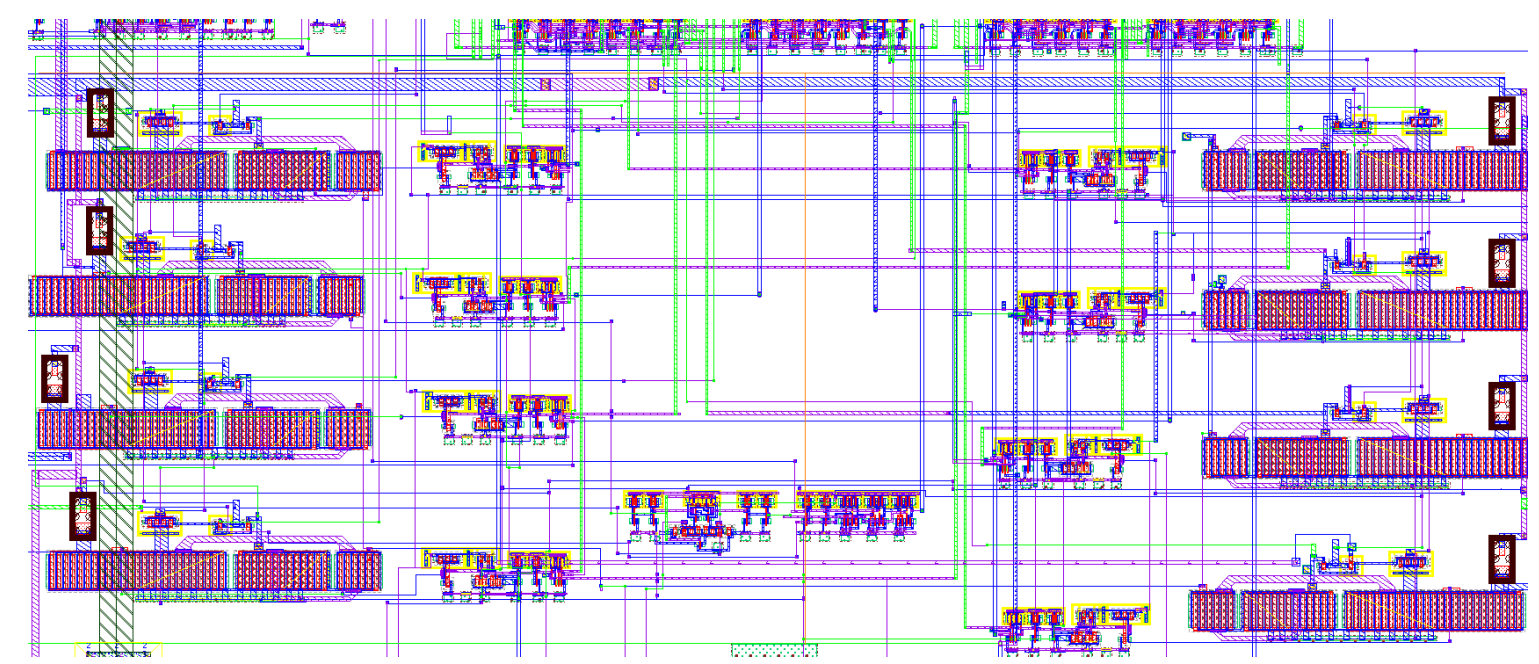

Figure 5.4: eFuse links and corresponding programming circuitry

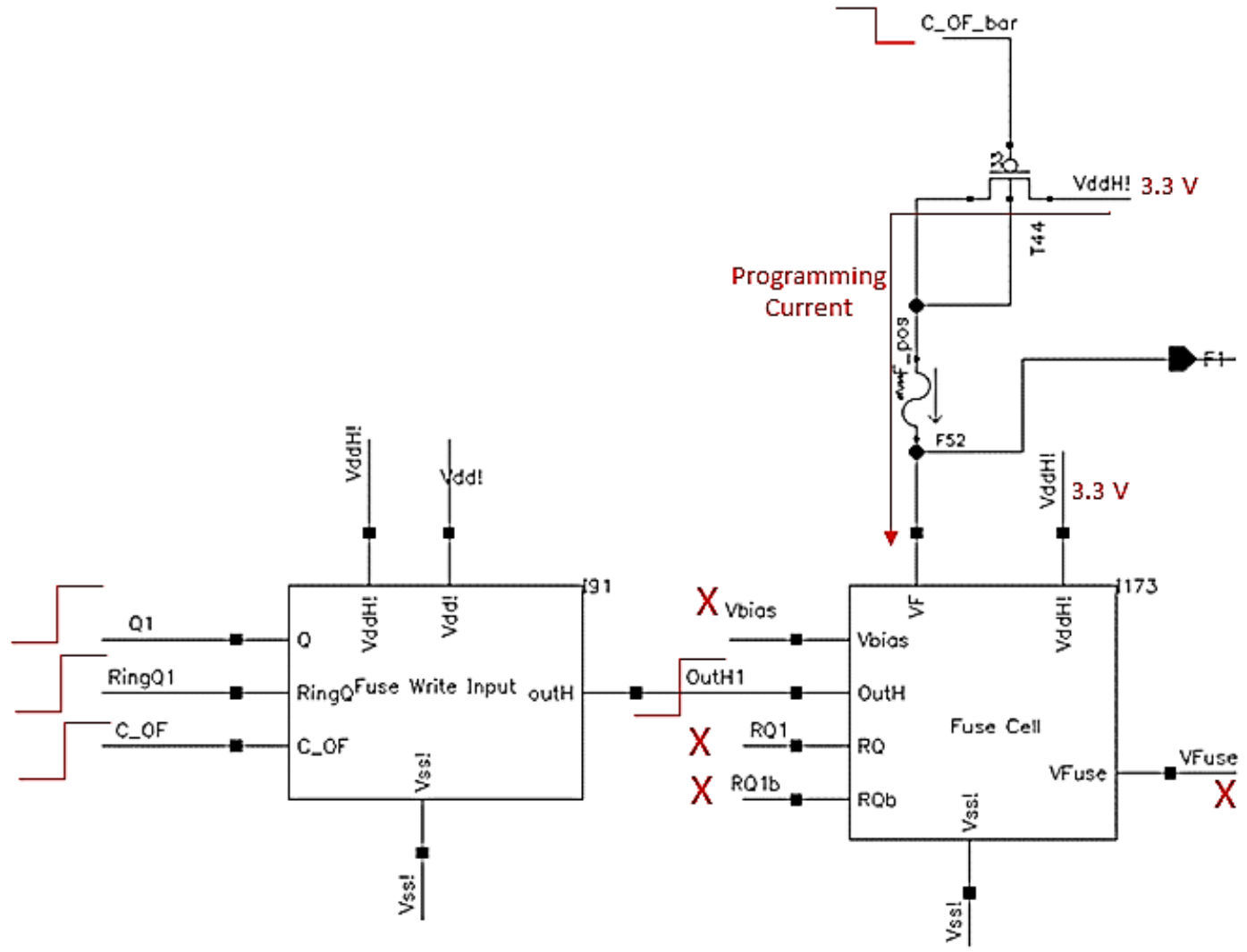

Figure 5.5: eFuse programming system block

flows as shown in Figures 5.5 and 5.6. 


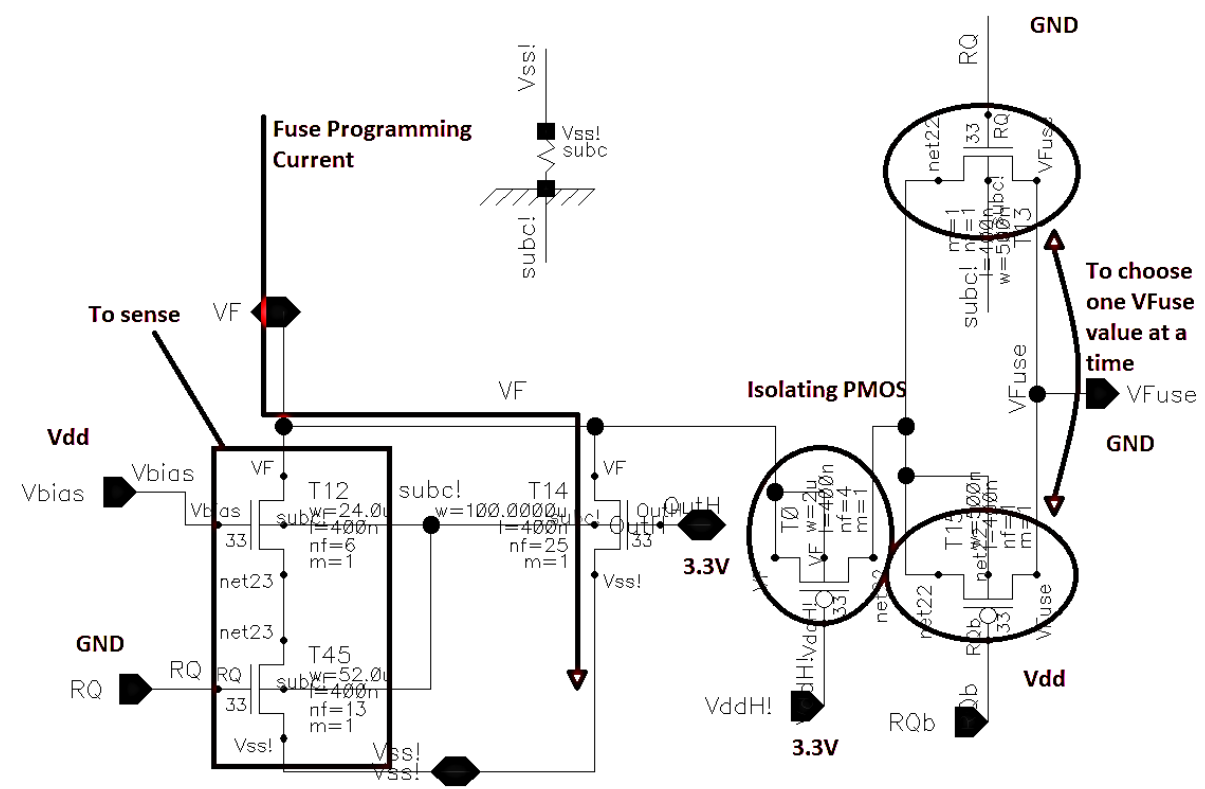

Figure 5.6: "Fuse Cell" circuit block which controls sensing and programming. Programming current path and signal conditions are shown here.

Figure 5.6 illustrates signal values in the case of programming an eFuse. $Q_{i}$ is any data bit passed to the registers; $R i n g_{Q i}$ is the output of a counter that starts up after the first 8 clock cycles. The counter's "1" output rotates through the eFuse cells one at a time. In other words, the eFuses can only be written in series due to large amount of current needed to program a fuse. The size of the program NMOS has been determined by simulations in order to pass a large current and the routing metals connecting this MOSFET to the eFuse and to the supply (3.3 V) and to ground have also been carefully sizes as per layout rules in order to avoid any electromigration or ground lifting, or voltage drop along them. $C_{O F}$ is the program enable signal which is set "High $=3.3 \mathrm{~V}$ " for programming and "Low $=0 \mathrm{~V}$ " for sensing. An isolating PMOS is placed between the sense/program circuit block and the VFuse output to the comparator to protect the comparator's input from the high voltages resulting from the programming step. The transmission gate only turns ON to sense the eFuses one at a time. The VFuse line is shared among the eFuses so eFuses need to be 
isolated from that line and only one value be loaded onto the line at a time. Figure 5.7 demonstrates a sample write operation signal pattern. It can be seen that 4 bits out of 8 are being burnt by applying $3.3 \mathrm{~V}$ which allows a current of $\approx 18.5 \mathrm{~mA}$ to pass through the fuses one at a time.

As shown in Figure 5.8, another isolating PMOS cuts off the $3.3 \mathrm{~V}$ supply from the low voltage side during programming. For sensing, as $V_{D D H}$ is grounded, $V_{D D}$ is connected through this PMOS to the eFuses. The size of this transistor has been chosen to minimize the voltage drop across its drain source. A transmission gate (i.e. an NMOS in parallel with this PMOS) would not have been possible since the aim of this design is to operate independent of a $3.3 \mathrm{~V}$ during sensing operation which relies on on-chip generated voltage.

Next, sensing operation is briefly explained.

\subsubsection{Reading Circuit Design}

Sensing operation involves the blocks shown in Figures 5.5, 5.8, and 5.9 in addition to a clock input, a clock phase shifter block and the output shift registers. Unlike other publications noted in the previous sections which rely on precharging the bitline directly connected to eFuses through access transistors, this topology precharges only the diffusion capacitances on nodes "X", "Y", "AA" and "BB" prior to sensing. Also, another benefit of this topology is that the best switching (latching) performance is achieved by using minimum size transistors. This is because the precharger does not have to drive a large capacitor resulting from many metal routings connecting eFuse nodes in parallel. Other benefits of using this circuit is that 1) it consumes zero static power, and 2) it directly produces rail-to-rail outputs. This topology was first 


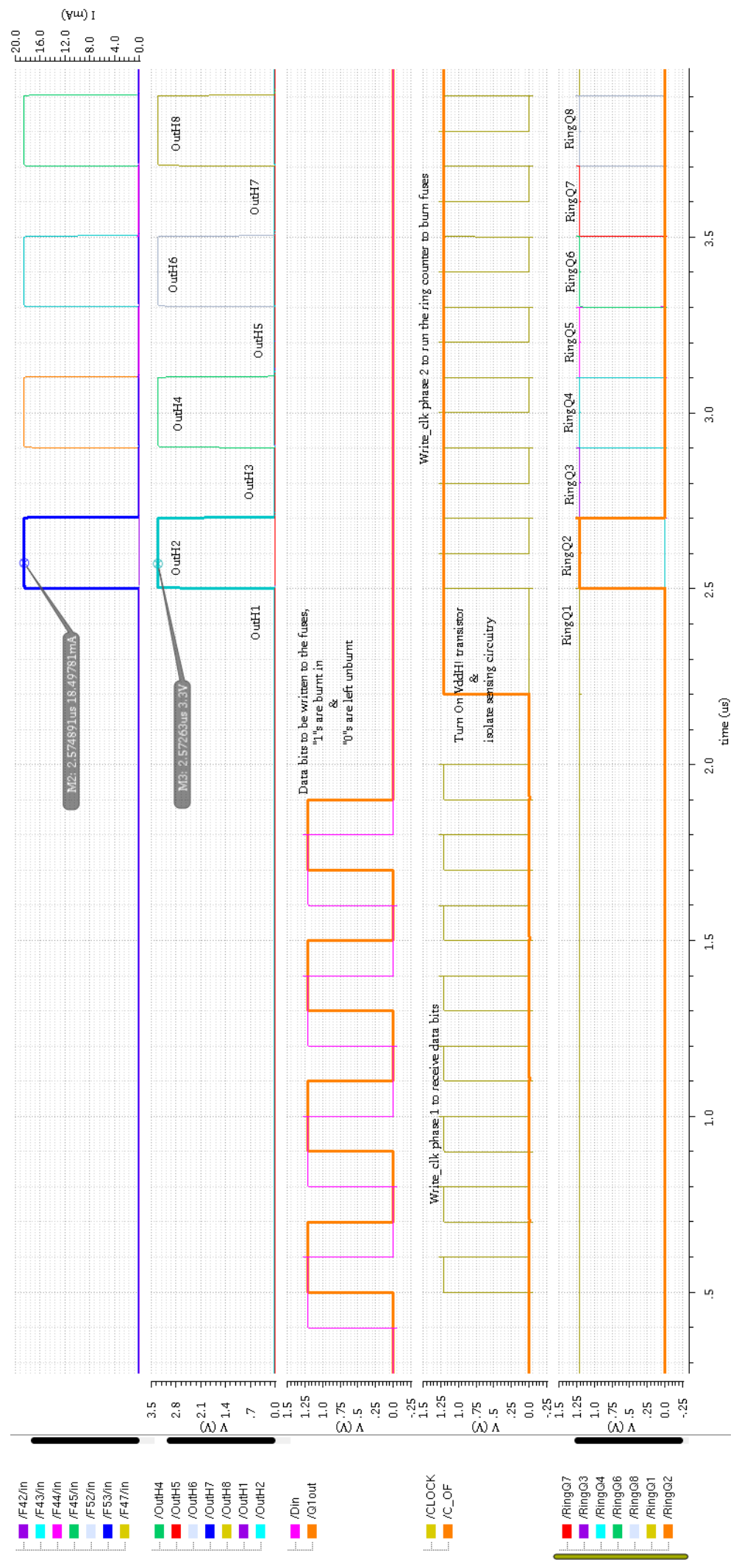

Figure 5.7: Fuse writing operation signal samples 


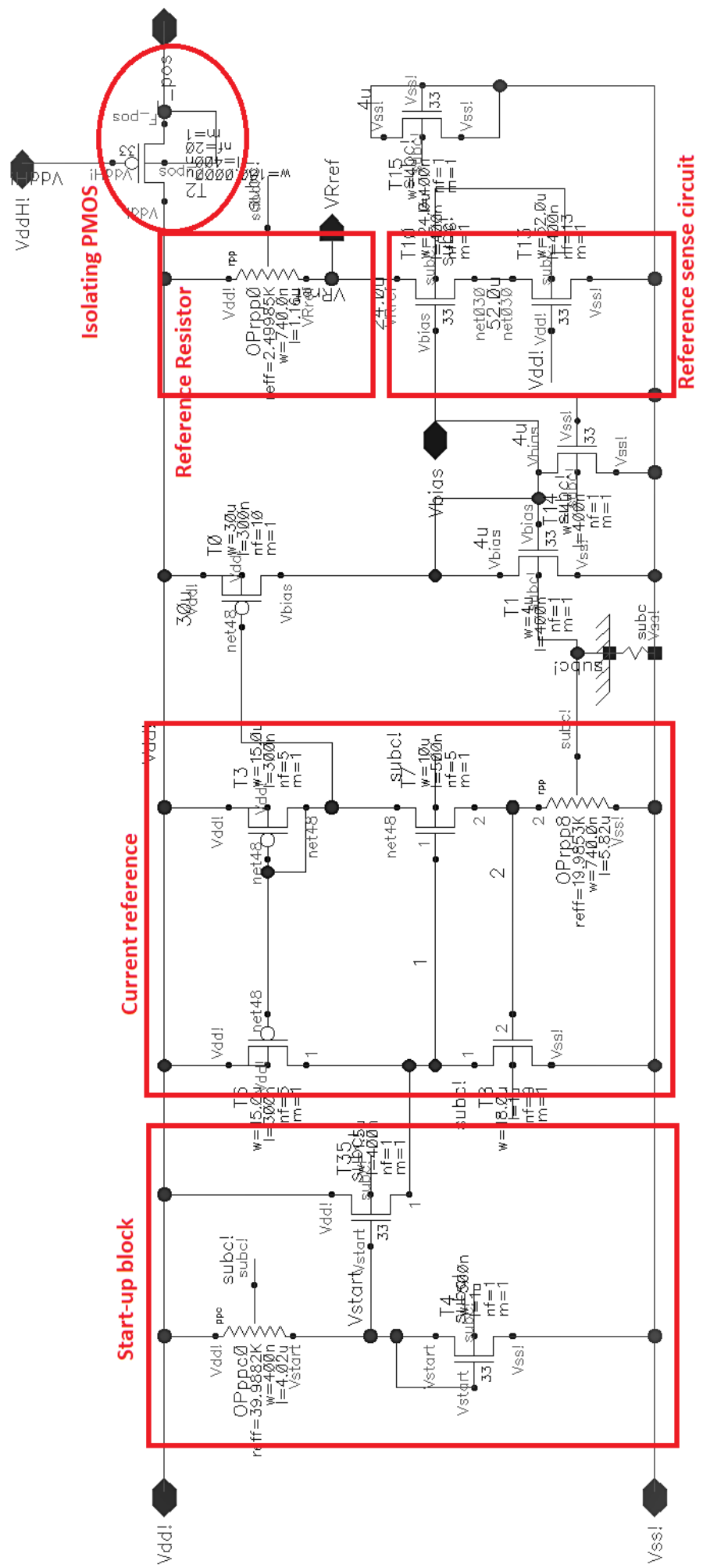

Figure 5.8: Supply independent current source for reading operation 
presented by [110] and further enhanced by [111]. Since this circuit relies on voltage gain resulting from the flow of charge from $C_{A A}$ and $C_{B B}$, the input offset must be minimum in order to increase the read sensitivity (i.e. the minimum input voltage difference detectable). This requirement puts an emphasis on a completely symmetric layout as seen in Figure 5.10.

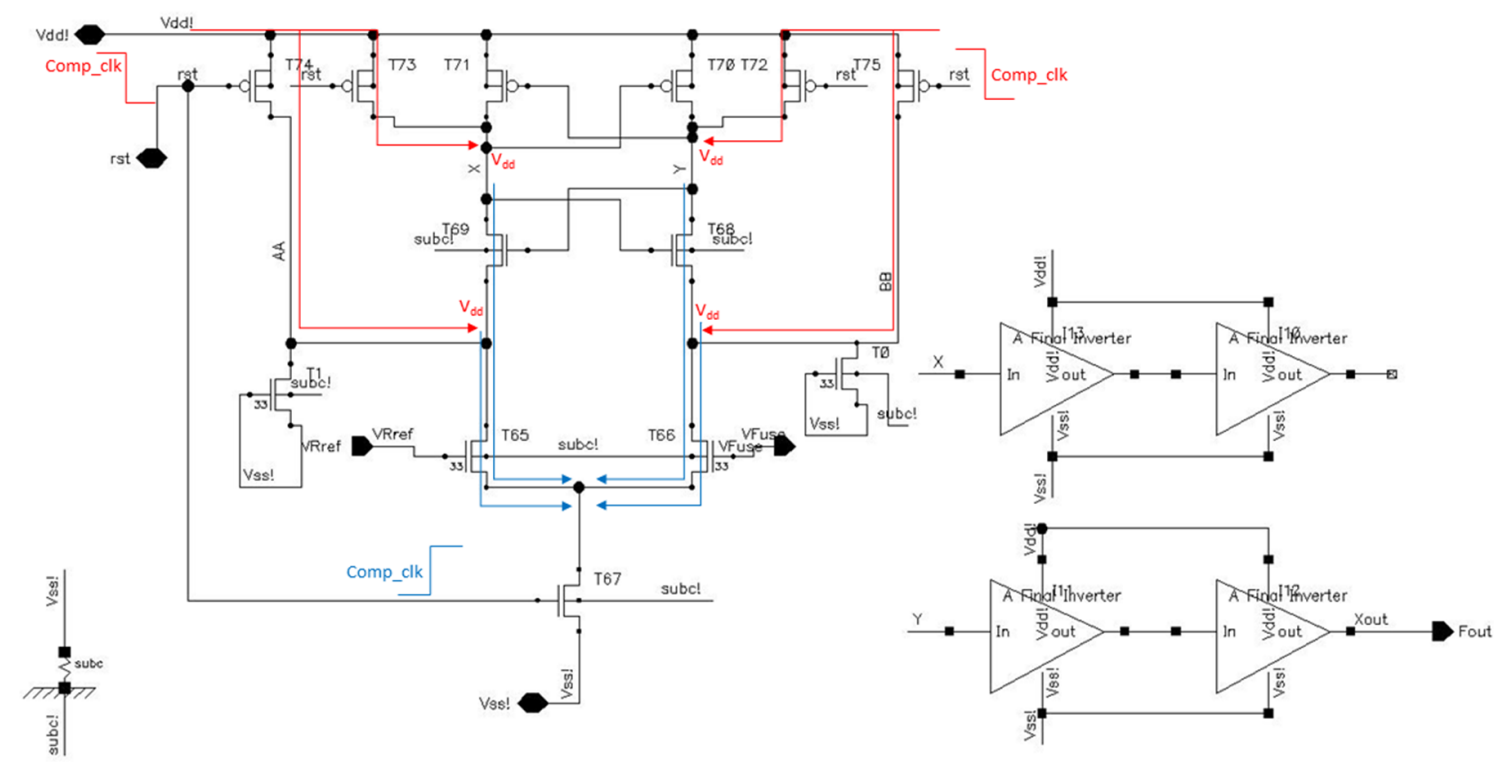

Figure 5.9: Clocked strong arm latch comparator for sensing

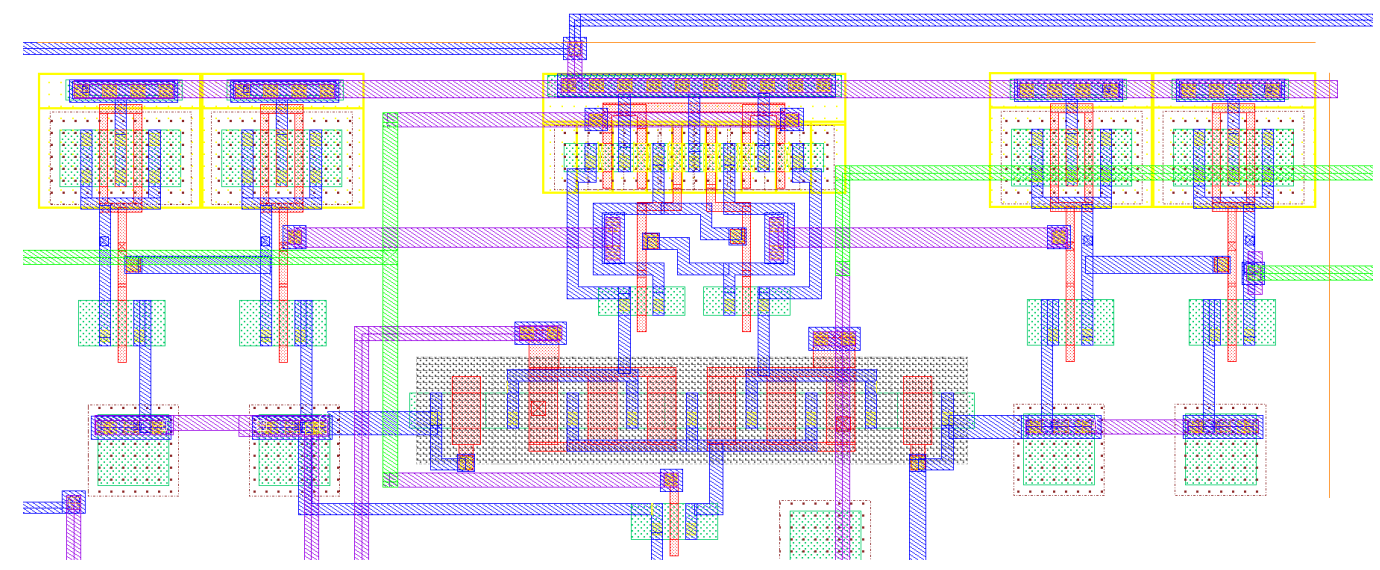

Figure 5.10: Readout block: The strong arm latch clocked comparator layout

A few considerations were taken into account when designing the sensing topology: 
1. High accuracy on-chip resistors where needed. Through simulation, it was determined that a few resistors used in the current reference circuit block and in the eFuse reference needed to be of high accuracy type. These polyresistors have a smaller process variation but also occupy a much larger footprint than low accuracy poly-resistors.

2. Choosing the reference resistor value. As per the technology documentations, a typical eFuse would have a resistance $\geq 5 \mathrm{k} \Omega$. Therefore, the reference resistor was set at $2.5 \mathrm{k} \Omega$, the approximate midpoint between $150 \Omega$ and $5 \mathrm{k} \Omega$. Monte Carlo simulations were performed to determine the trip point - closet value to the reference resistor correctly detectable. Simulation results will be included later.

3. Conservative design approach vs. power consumption. The lower the reference resistor value, the higher the bias current running through the reference and the eFuses. While it is possible to assume a higher programmed eFuse resistance, the decision was made to design conservatively for the sake of robust and reliable operation. Further iterations of this design would minimize footprint and power consumption.

Figure 5.11 shows the logical signal flow for a read operation. As the Comp_clk goes HIGH, the precharging PMOS's turn off and the evaluation period begins by turning on the NMOS as shown in Figure 5.9. The two signals being compared are at the inputs of the differential pair called VRef and VFuse. It can be seen from Figure 5.11 that the two signals are set up properly before the evaluation period in order to ensure stable signal read operation.

As the read_clk signal rises, the Comp_clk rises, and since the VFuse is lower than 


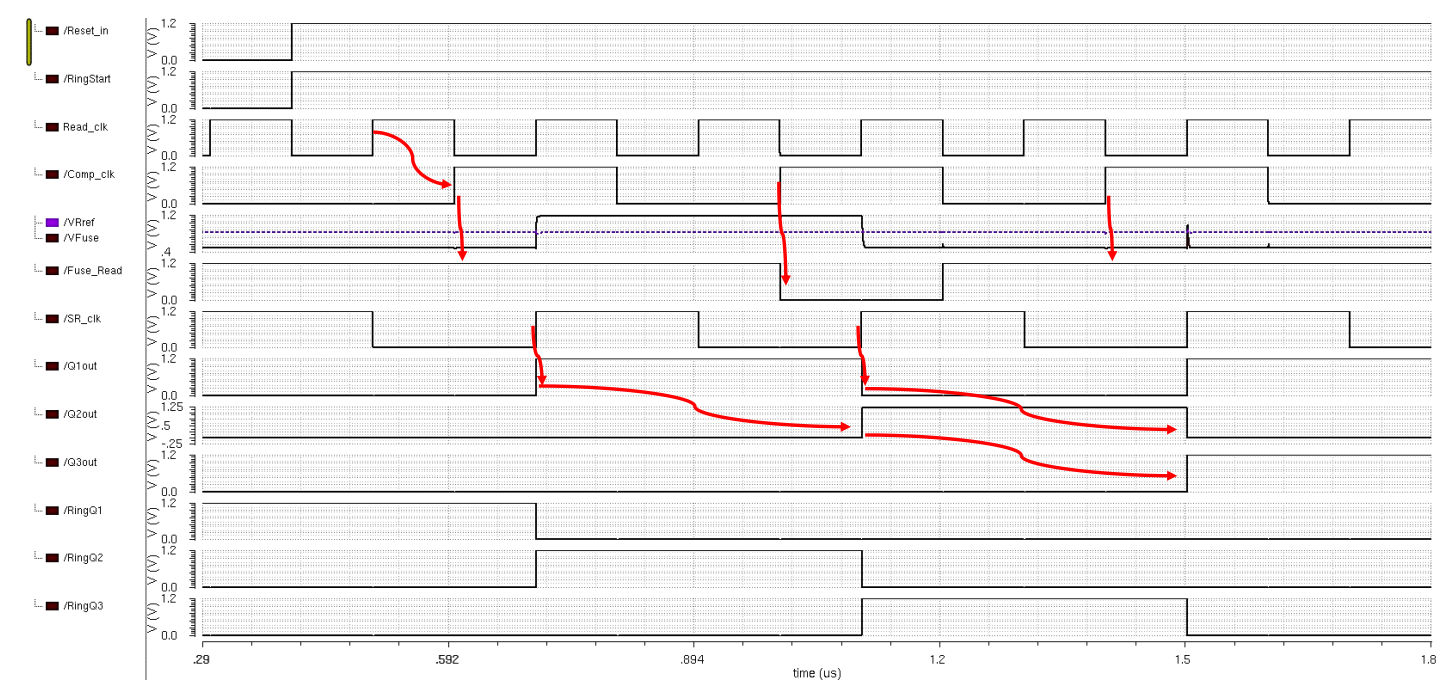

Figure 5.11: Read operation signal flow

the VRef, there is no transition in the Fuse_Read signal. As the first $S R_{-} c l k$ positive signal edge arrives, it passes the "1" to the first register Q1out. This process during the RingQ1 signal which enables fuse 1 to be read. If VFuse $<$ VRef, then the fuse has been blown (high resistance) which is interpreted as a logical "1", otherwise it is a logical " 0 ". As RingQ1 goes low and RingQ2 goes high, fuse 2 is read which transitions VFuse from low to high and the rest of the operation repeats as before.

As the supply voltage is reduced from the nominal $1.2 \mathrm{~V}$ to $0.53 \mathrm{~V}$, the difference in voltage between VFuse and VRef reduces to the point where reliable read operations are not possible. The current source circuit shown in Figure 5.8 (repeated in Figure 5.12 for clarity) is the essential block to maintain a difference between the two signal voltages within the supply range. Individual transistors' DC current values are shown in Figure 5.13 for the case of a "connected" fuse and those for a "blown" fuse are shown in Figure 5.14. Figures 5.15 and 5.16 illustrate the difference between VRef and Vfuse as the supply voltage changes for the connected and blown fuse cases respectively. As can be seen, except for the curves shown in "purple" and "blue" all 


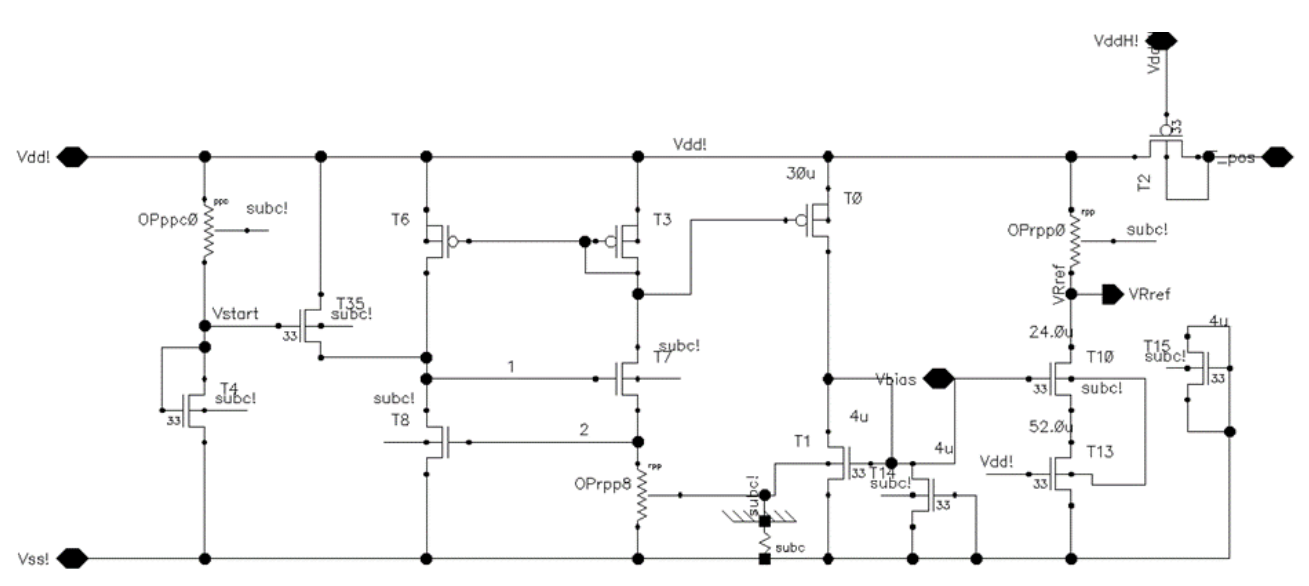

Figure 5.12: Supply independent current source

the other currents are fairly unchanged throughout the range of supply voltages. The dotted black curve shows the entire chip's current draw. The transistors' currents in Figure 5.13 correspond with the transistors in Figure 5.12.

"T2" and "T10" current curves are not constant with supply voltage. "T10" s gate is biased internally which would make it draw current independent of the supply much like other transistors in the circuit. It was decided to remove a PMOS transistor where the "Reference Resistor" in Figure 5.8 is placed. This causes the current in that branch not to be perfectly independent of the supply. If the PMOS where to be placed instead of the reference resistor, then VRef would stay unchanged with supply, but on the other hand, the Fuse circuit structure would not be an exact mirror of that of the reference. More over, with a PMOS in place, VRef would drop to only 10's of millivolts where there would be no comparison between each fuse and the reference circuit. Also, a reference resistor $(2.5 \mathrm{k} \Omega)$ mid-way in value between a connected fuse $(100 \Omega)$ and a blown fuse $(5 \mathrm{k} \Omega$ and more) would result in a more accurate comparison than a reference PMOS. 


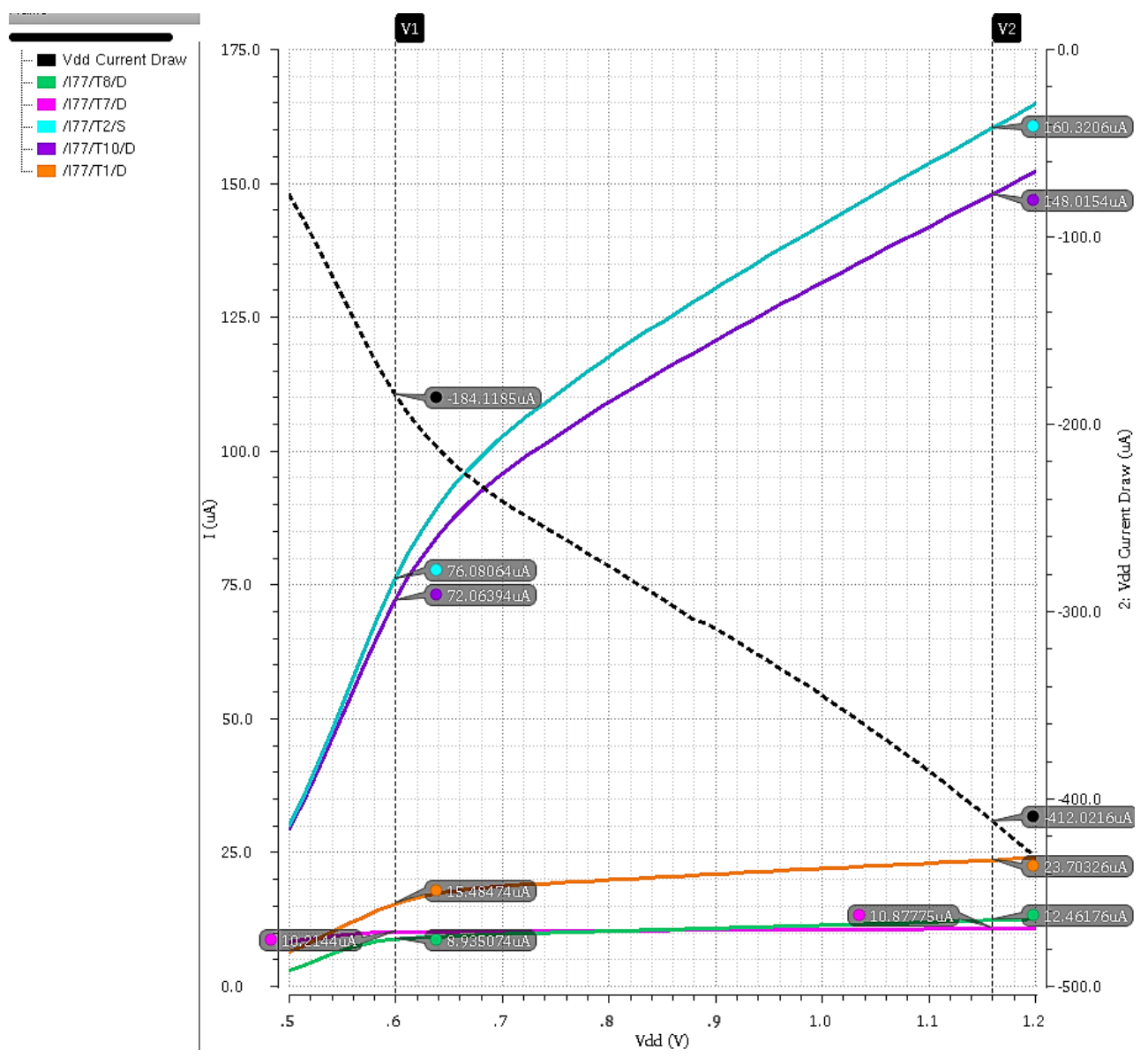

Figure 5.13: DC current flow through the current source block for reading of a low resistance (connected) fuse

Many iterations of post-layout Monte Carlo simulations and corner plus temperature simulations were performed in order to arrive at the best possible solution to the reference vs. fuse resistor comparison. This primary design iteration has in mind the correct and optimum (in term of power consumption) operation of the memory chip as a whole than maintaining the current in the reference and fuse branches. The limitation arises from the fact that a common branch feeds both the sensing and writting currents to the individual fuse, which means in order to pass $15 \mathrm{~mA}$ of current through a PMOS, sizes above $100 \mu \mathrm{m}$ are needed for each fuse which would not be efficient in area. Future design iterations would consider other methods to try to 


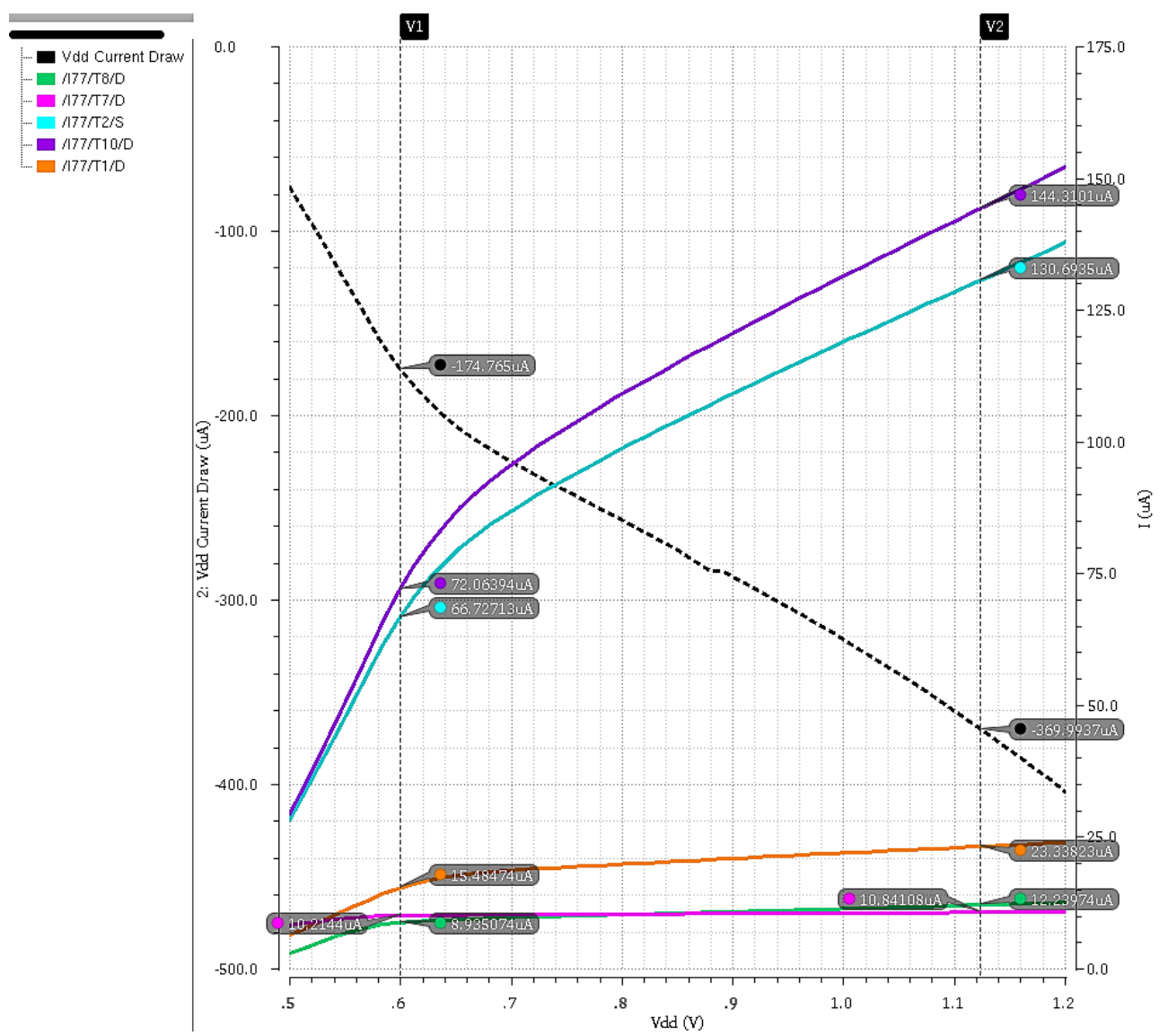

Figure 5.14: DC current flow through the current source block for reading of a blown (high resistance) fuse

make the current in that branch independent of the supply while maintaining the same $\mathrm{read} /$ write robustness and reliability and optimum power consumption presented in the current design.

\subsubsection{Layout}

The system layout had to be manually drawn since no automatic layout tool was available which could reliably implement the routing and also pass DRCs. There is always room to improve the layout and to make it more compact but since the aim of this research work is to implement a robust low power design and no area reduction criteria was set, this design has already achieved its objective. Most of the area 


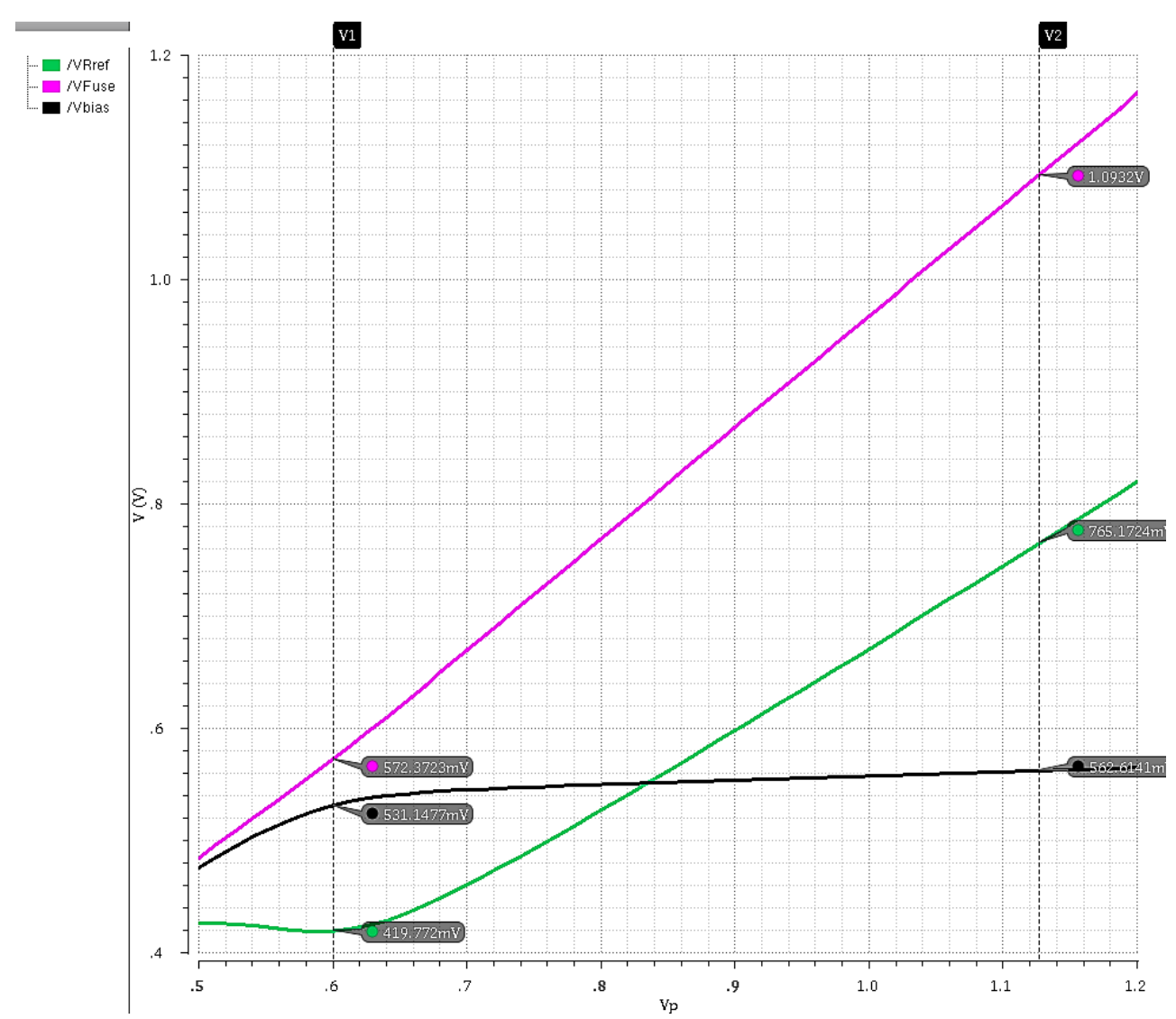

Figure 5.15: DC values of VRef, VFuse, and Vbias in the case of a low resistance fuse as per $V_{\text {DD }}$ sweep

has been taken up by the programming transistors since they need to draw a large current. Metal routings were designed having current density and resistance in mind. Every digital block included in flip flops, registers, buffers, etc. were designed and simulated to ensure reliable operation and power consumption. Figure 5.17 illustrates the layout of the memory block.

Next, testing of the memory chip is described. 


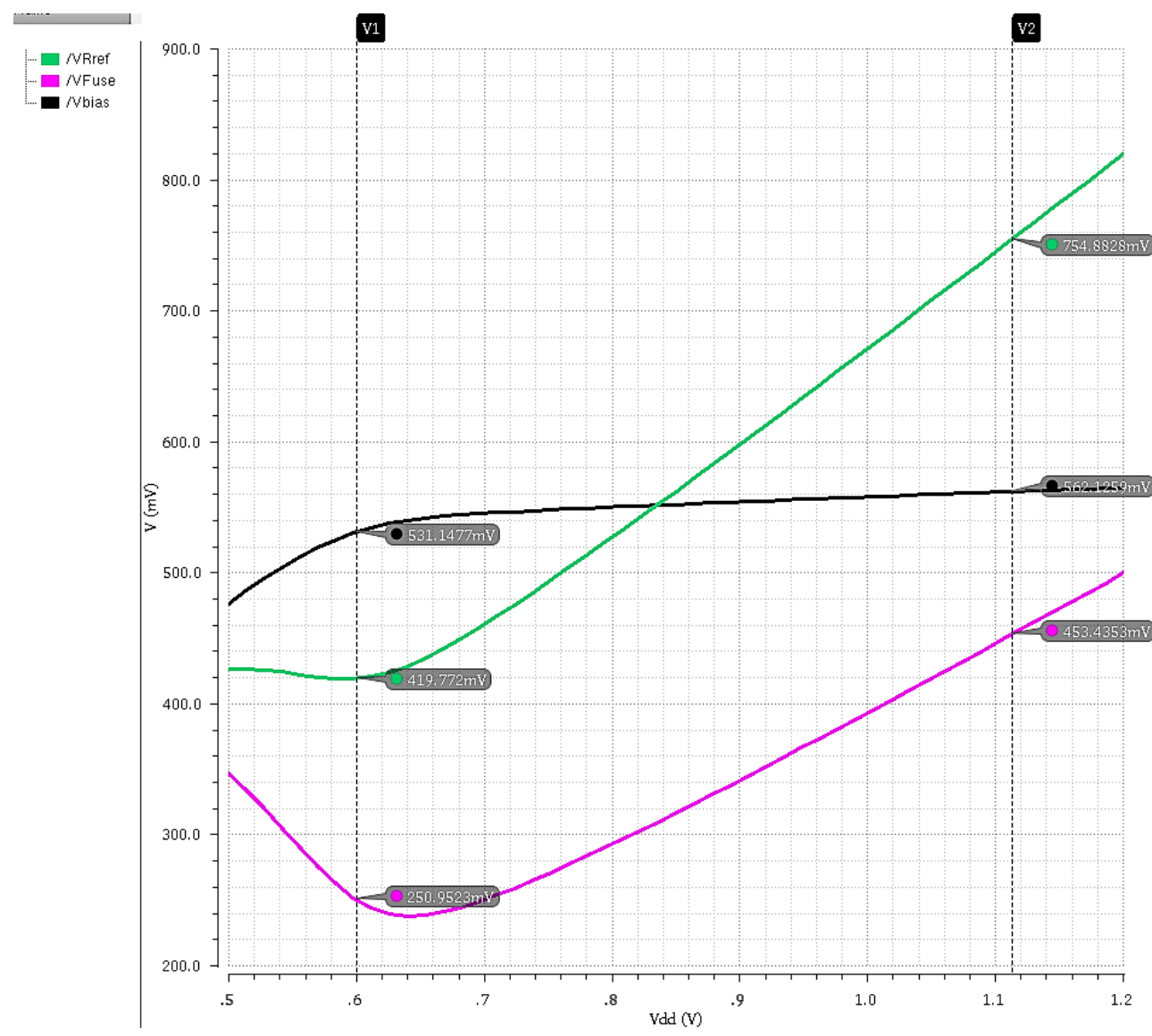

Figure 5.16: DC values of VRef, VFuse, and Vbias in the case of a blown (high resistance) fuse as per $V_{\mathrm{DD}}$ sweep

\subsubsection{Testing}

The memory block was successfully tested using a Digilent Analog Discovery which generates the necessary input signals to program or sense the memory. The test setup, sample waveforms, read/write signal passing, and output signal pictures are presented in Figures 5.18, 5.19, and 5.20. The additional resistors and capacitors seen in Figure 5.19 are responsible for voltage division of the output signals from the signal generator (since digital I/Os are at $3 \mathrm{~V}$ ) to chip input signals of $\sim 0.5-0.6 \mathrm{~V}$ and power supply decoupling and surge protection, respectively. 


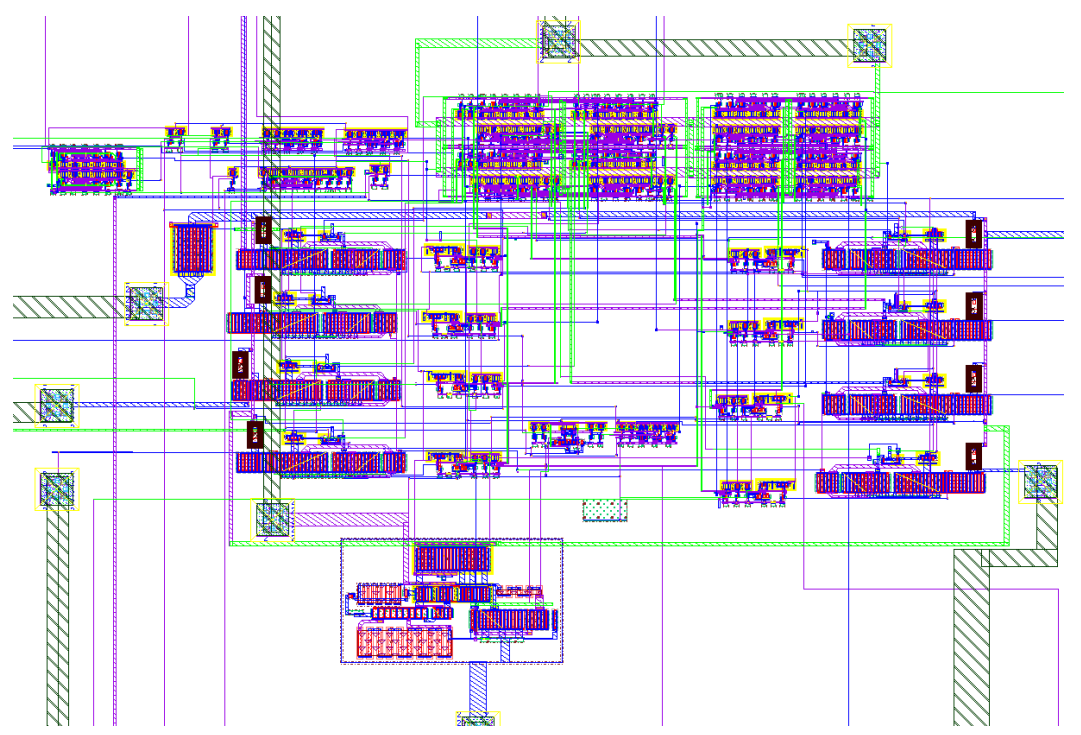

Figure 5.17: Zoomed-in layout of the ID block

The robust operation of the memory block has been tested under X-ray radiation using the Raycell MK2 blood irradiator. The packaged memory chip was installed on a breadboard with all the input signals attached via a CAT 7 shielded cable and passed through the opening from behind the irradiator machine. While under radiation, the memory block was read and the correct bit stream previously programmed on the chip was output. The supply was kept at the minimum $\sim 0.5 \mathrm{~V}$. A total radiation dose of 150Gy was applied by the machine during which the memory chip was read continuously to ensure correct operation. 


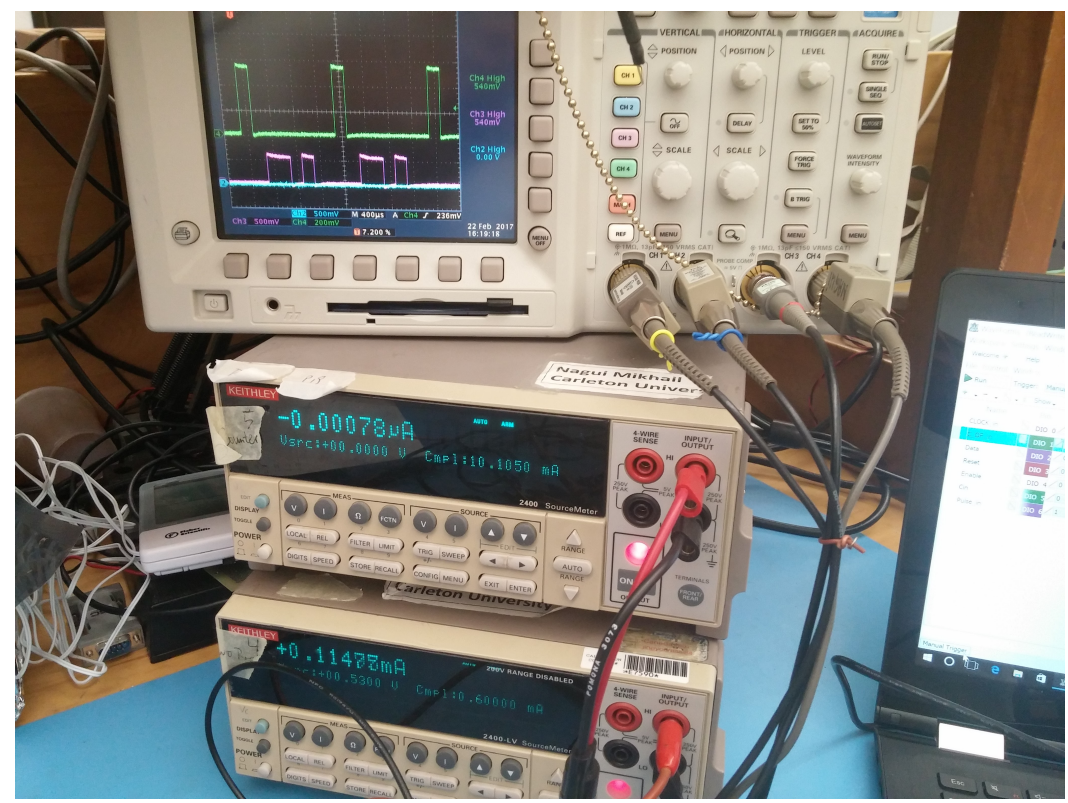

Figure 5.18: A read operation sample illustrating average power consumption of $60.81 \mu \mathrm{W}$

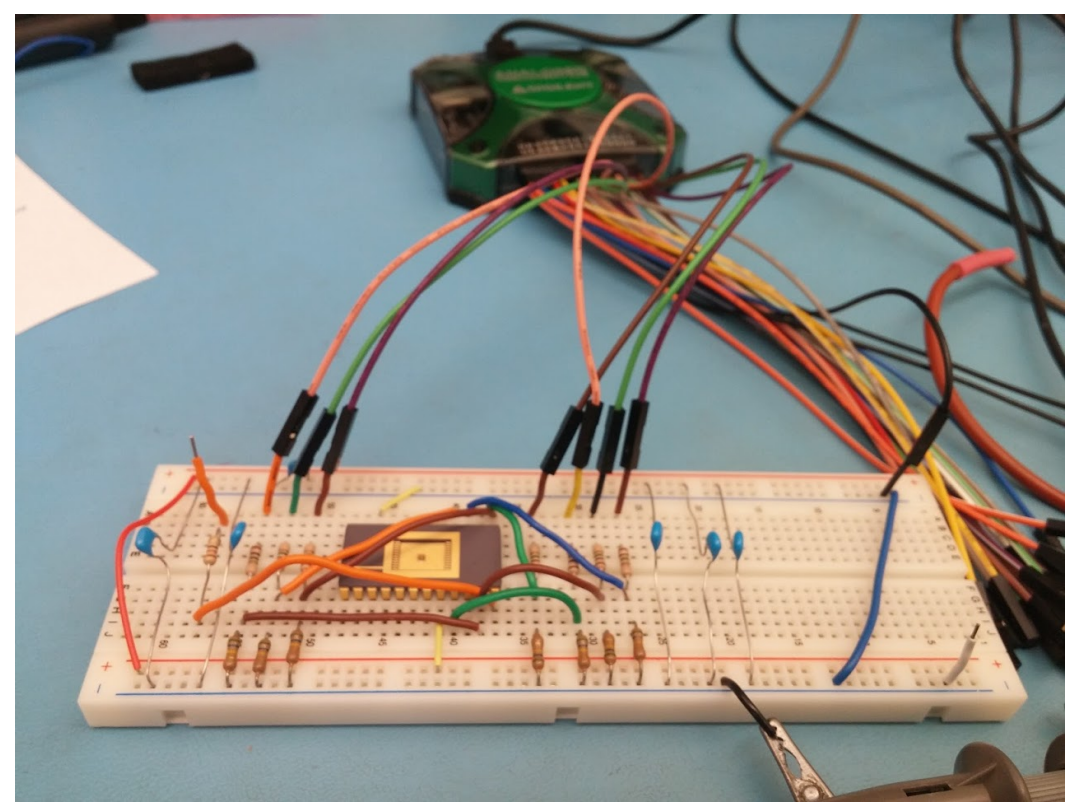

Figure 5.19: Memory test setup illustrating a set of resistor dividers in order to adjust the input signal voltage levels from nomial $3 \mathrm{~V}$ output from the signal generator to $0.5 \mathrm{~V}$ at the chip input. 


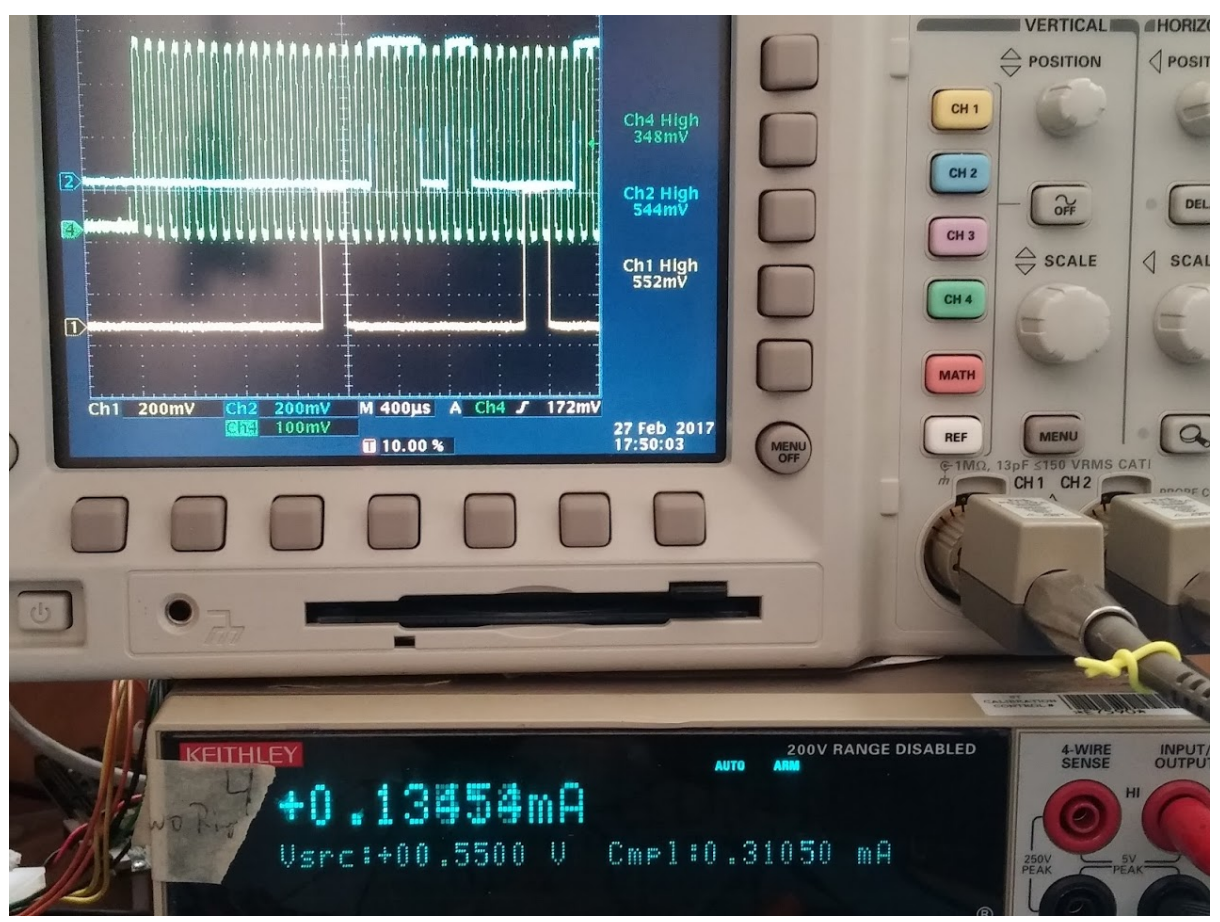

Figure 5.20: Clock signal supply reduction and operation of the chip in read mode 


\section{Chapter 6}

\section{Thesis Conclusions, Contributions, and Future Work}

\subsection{Thesis Conclusions and Contributions}

This thesis has made several notable advancements to the field of ultra-low power wireless dosimetry using a standard RF-CMOS technology. In addition, this work has furthered the wireless X-ray dosimeter project at Carleton University. The main contributions are summarized below.

With regards to objective 1:

A thorough investigation and full characterization of floating-gate MOSFETs developed in a standard RF-CMOS process for the application of X-ray dosimetry in blood irradiation has been presented. The feasibility of using such MOSFET devices as floating-gate sensors is successfully demonstrated through X-ray irradiations using two different ranges of X-ray photon energies. It was successfully demonstrated, for the first time, that removing the $\mathrm{CoSi}_{2}$ layer from the floating-gate extension can lead to increased radiation sensitivity by lowering the energy barrier at the oxide-Poly-Si 
gate interface. This was verified by irradiation with two X-ray sources.

It was shown that the presence or the absence of a metal shield on the floating gate does not affect the sensitivity. Also, sensitivity does not vary significantly in the multi- vs. single-finger configurations of the FG-PMOS devices.

A complete characterization of the charging and discharging operations of the FGPMOS presented helps pave the path for an optimum automated precharging circuit block. Many charge/discharge cycles performed with different pulse widths and heights have also proven that the gate oxide can withstand such operations without any significant degradation in charge retention.

With regards to objective 2 :

A novel concept never explored before in radiation dosimetry has been presented. Subthreshold dosimetry has the potential to push the boundaries of low power wireless radiation sensing. Utilizing the X-ray sources available to us has shown that radiation sensitivity levels are not high enough compared to FG-PMOS and FG-NMOS devices, considering that the gate oxide thickness is too small. Numerous circuit configurations could be developed using this concept, aiding in technology advancements in lowpower radiation dosimeters that do not rely on charge depletion of a floating gate.

With regards to objective 3 :

A novel minimum size FG-inverter is designed, implemented, and successfully irradiated. This design helps further push the boundaries of integrated dosimeter sensor design with extremely low power consumption. While the sensor practically has 1 bit of resolution, meaning it indicates its depletion of charge by flipping the output, its concept of operation can be expanded to other digital designs with more resolution. Also, in this design, it was demonstrated that by precharging the device with lower 
or higher amount of charge, one can set the transition point of the output in order to set the desired level of irradiation exposure.

With regards to objective 4:

A novel approach to X-ray energy harvesting is presented using discrete diodes. These standard silicon p-i-n diodes showed great potential as a means to power the dosimeter while under irradiation, a concept never explored before. While the diodes themselves generate insignificant amounts of power, employing scintillating materials showed a great increase in their power conversion efficiency. One scintillating material showed the most increase in useful power generation. It was also shown that series or parallel combination of these photodiodes can multiply the power generated. Having introduced minimum sized FG-inverters as dosimeters with minimum power consumption, a combination of photodiodes and FG-inverters could be used as a self-powered radiation dosimeter.

With regards to objective 5:

A low power, radiation-insensitive 8-bit OTP memory for chip-ID has been developed in the same $0.13 \mu \mathrm{m}$ RF-CMOS process. Its unique design allows it to operate in a supply range of $0.53 \mathrm{~V}$ to $1.2 \mathrm{~V}$, hence enabling operation in conditions where the supply voltage is provided by a depleting supercapacitor, a weak wireless power, or from the diode harvester. The novel design isolates the high voltage programming circuit block from the sensing circuit block in order to protect the low voltage transistors during the write operations. This circuit block will be integrated into the dosimetry system in the future.

Full read/write characterization has been successfully performed and the block has passed the test of sensing (read) operation under X-ray irradiation for a cumulative 
dose equivalent to four times that necessary for a blood bag irradiation. It occupies a fraction of the entire chip area and consumes only $60 \mu \mathrm{W}$ of power for the duration of its operation.

\subsection{Future Work}

Further areas of research opened up as a result of studies in this thesis are suggested here:

\subsubsection{Future Work on FG-PMOS Devices}

With respect to FG-PMOS sensors, future experiments could include:

1. Full characterization of the radiation response of the same devices under ${ }^{60} \mathrm{Co}$ and ${ }^{137} \mathrm{Cs} \gamma$-ray exposures. The investigations would include response reproducibility and possible degradation.

2. Integration of the devices in low power direct readout circuit blocks such as in ring oscillators ( $\mathrm{RO}$ ) as a current source with a decreasing channel current with increasing cumulative radiation dose. Such a circuit block can be used as a radiation-to-frequency converter since a decrease in current drive in the RO means a decrease in its frequency. The study of such circuits could be of interest due to nonlinear behavior of FG-PMOS radiation sensor with radiation and the nonlinear behavior of the RO with changes in its current drive. The analysis of the combination of these nonlinear behaviors could lead to better sensor readout designs.

3. A reference FG-PMOS can be used to remove first order process and temperature variations in an identical circuit block but with the gate biased. 
4. The complete precharging/discharging characteristics of the FG-PMOS presented would enable the future development of the automated precharging mechanism as a crucial step towards complete integration of the sensor. Current designs utilize externally generated pulses, but with the outcome of this investigation, the optimum pulse height and width for precharging and discharging of the FG-MOS has been determined which would be used as design criteria for a low power on-chip pulse generator circuit block.

\subsubsection{Future Work on Integration and Functionality En- hancements}

Successful demonstration of a novel FG-inverter dosimeter in a commercial fabrication process sets the stage for further development of a variety of digital circuit blocks that could sense radiation without consuming static power, unlike mainstream dosimeter designs which rely on constant monitoring of channel current to determine the cumulative dose.

Presented below is a novel digital dosimeter with no static power consumption. The circuit is already designed, laid out, and is awaiting fabrication.

\section{A Novel Clocked Comparator-Based Digital Ionizing Radiation Dosimeter With 4 Bits of Resolution}

A comparator based on the strong arm latch design of [111] is presented in Figure 6.1. The original design uses NMOS transistors as input differential pairs whereas here the PMOS devices are used. The MOS labeled with $16 \mu \mathrm{m}$ is the FG-PMOS device and the 4 MOS's on the left hand side are connected to a binary up counter

which turns them on by applying a "low" signal to their gates. 16 bits of resolution 
are hence created that could track the change in the FG-PMOS gate charge (voltage) indirectly. The bit pattern that flips the output is taken as the equivalent gate charge left on the FG. This procedure repeats constantly during the irradiation and once the session is over, the bit pattern that changes the output is taken as equivalent to cumulative dose.

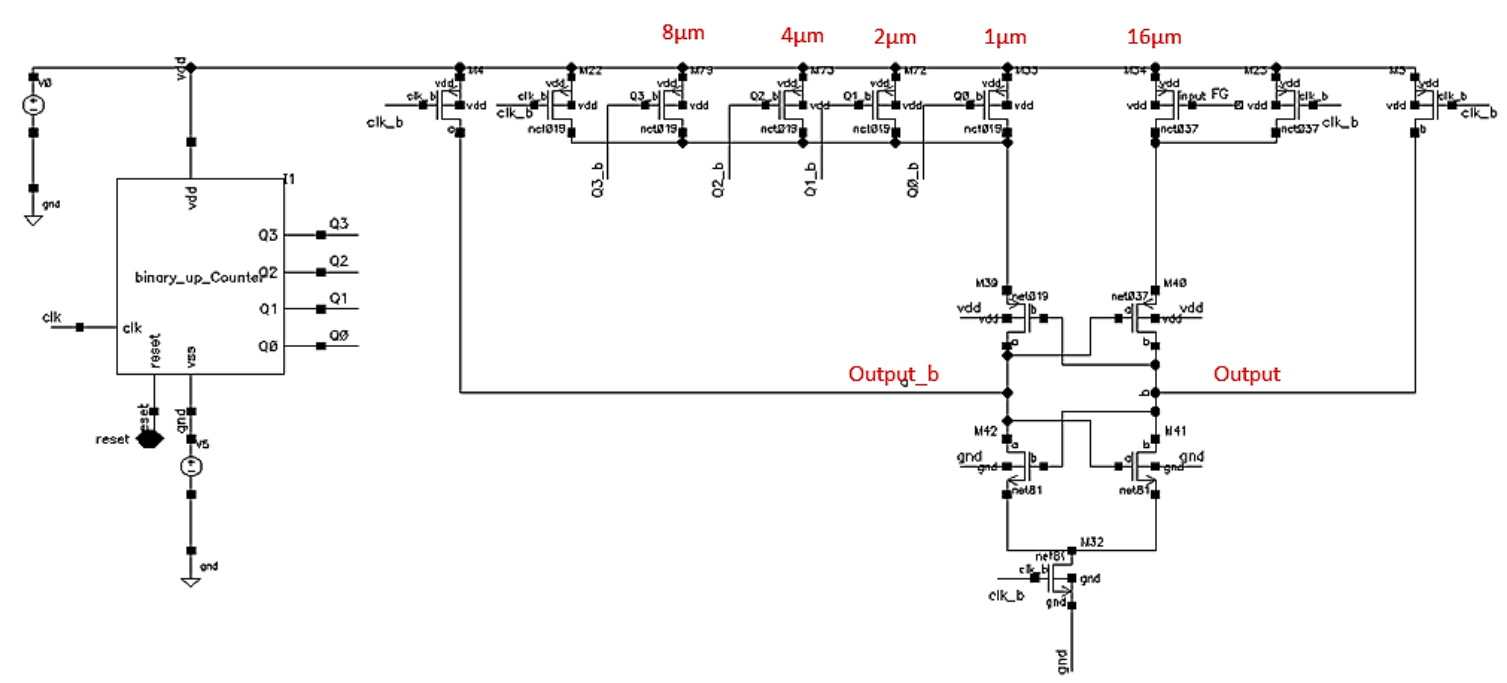

Figure 6.1: Comparator based 4-bit resolution FG-dosimeter with zero static power consumption

Since the operation of the comparator is tested successfully in the design of the NVM in Chapter 5, and the FG-PMOS has been successfully demonstrated to be sensitive and stable under irradiation, there is no doubt as to the successful combined operation of the two. It is worth mentioning that the clock signal charges the internal nodes of the comparator prior to each sequential step of the binary up counter. The only power consumption is from discharging the internal nodes of the comparator on each clock cycle. To reduce the power consumption, the up counter and hence the node precharging stage can be done at a lower frequency.

Also, the supply voltage of the whole block can be reduced to $\approx 0.5 \mathrm{~V}$ as was the case 
in the NVM design, which would further reduce power consumption.

\section{Diodes As X-ray Power Harvesters}

As shown in Chapter 4, discrete diodes were successfully demonstrated to generate useful power from irradiation by coating them with scintillating materials.

Further research into other scintillating materials would surely be able to increase the power conversion efficiency of the diodes. Currently, $\mathrm{CdWO} \mathrm{O}_{4}$ and $\mathrm{PbWO}_{4}$ have been studied and chosen for the future investigations into higher power generation under radiation.

Furthermore, four varieties of on-chip diodes which are already fabricated but not tested under radiation should be tested. These varieties are of much smaller dimensions but their expected power generation efficiency could be extrapolated from the results from the discrete diodes.

Once finalized, these diodes could in future designs be used to drive low power, low voltage digital dosimeter circuit blocks introduced above. In terms of cost of implementing photodiodes on chip or using the discrete variants explored in Chapter 4, it is important to keep in mind the intended use of a radiation-powered dosimeter. For some applications, the increase in cost incurred by implementing the photodiodes on chip may be worthwhile, whereas in some other applications where mass production is considered, lower cost must be maintained and hence discrete diodes may be preferred.

\subsubsection{One-Time Programmable Nonvolatile Memories}

The complete read/write design introduced in Chapter 5 should be integrated into the dosimeter system. Moreover, further optimizations in its design can improve power 
consumption even more. The use of anti-fuse devices introduced in the literature review would need to be investigated as an option.

\subsubsection{Publications}

Expected publications from this work are listed below:

Refereed Journal Articles

1. B. Yadegari, et. al., "A novel X-ray CMOS Based Ultra-Low Power Wireless Dosimeter System for Blood Sterilization Applications", IEEE Journal on Circuit and Systems, 2019 (under preparation)

2. B. Yadegari, et. al., "Low power Floating-Gate PMOS X-Ray Radiation Sensor", IEEE Transactions on Electron Devices, 2019 (under preparation)

3. B. Yadegari, et. al., "Ultra-Low power Chip ID Block Developed for X-Ray Radiation Sensor for Blood Sterilization", IEEE Transactions on Electron Devices, 2019 (under preparation)

4. B. Yadegari, et. al., "A Novel X-Ray Radiation Power Harvester Block Utilizing On-Chip PN Junctions", IEEE Transactions on Nuclear Science, 2019 (under preparation)

To date, one conference publication has appeared:

B. Yadegari, O. Sanusi, F. A. Ghaffar, A. Shamim, L. Roy and A. Shamim, "An Efficient and Compact Wireless Solution for Blood Sterilization Apparatus", IEEE International Symposium on Antenna Technology and Applied Electromagnetics (ANTEM), (August 2018). 


\section{List of References}

[1] N. Schroeder. (2016) Sensors markets 2016: Development of sensors markets for the non-military, open world market until 2016. [Online]. Available: http://www.intechnoconsulting.com/ic/press/details/e-press-se2016-us.pdf

[2] P. Danini. (2013) Steady growth and technology in ections open opportunities for medical image sensors. [Online]. Available: http://www.i-micronews.com/ upload/pdf/MEMSTrendsJanuary2013AC.pdf

[3] —. (2011) Frequently asked questions - products. [Online]. Available: http://www.hc-sc.gc.ca/ewh-semt/occup-travail/radiation/dosim/ res-centre/faqprod-eng.php

[4] J. Font, J. Cesari, and A. Pineda, "Radiation Sensor With Current Output," vol. 60, no. 5, pp. 4026-4030, 2013.

[5] (2000). [Online]. Available: http://www.hss.gov.nt.ca/sites/default/les/ keyfactsaboutbloodtransfusions.pdf

[6] J. T. et al. (2011) Guidelines on the use of irradiated blood components prepared by the british committee for standards in haematology blood transfusion task force,. [Online]. Available: http://dx.doi.org/10.1111/j.1365-2141.2010.08444.x

[7] P. I. Sheet, "Process Verification for Blood," 2008.

[8] B. theratronics: (2013) Gammacell 1000 elite/3000 elan brochure. [Online]. Available: http://www.theratronics.ca/PDFs/BT_MBTS_8005_ GC1000E_3000E_3_V112013_webSECURE.pdf

[9] B. Theratronics. (2013) Raycell mk2 brochure. [Online]. Available: http://www. theratronics.ca/PDFs/RCMK2_BTMBTS_8014RCMK2_4_v112011_web.pdf 
[10] C. J. Peters, N. G. Tarr, K. Shortt, I. Thomson, and G. F. Mackay, "A floatinggate mosfet gamma dosimeter," Canadian Journal of Physics, vol. 74, no. s1, pp. 135-138, Dec 1996.

[11] N. G. Tarr, G. F. Mackay, K. Shortt, and I. Thomson, "A floating gate mosfet dosimeter requiring no external bias supply," in RADECS 97. Fourth European Conference on Radiation and its Effects on Components and Systems (Cat. No.97TH8294), Sep 1997, pp. 277-281.

[12] N. G. Tarr, K. Shortt, Y. Wang, and I. Thomson, "A sensitive, temperaturecompensated, zero-bias floating gate mosfet dosimeter," IEEE Transactions on Nuclear Science, vol. 51, no. 3, pp. 1277-1282, June 2004.

[13] M. Arsalan, E. Carleton University. Dissertation. Engineering, and Computer, "Wireless system-on-chip sensor design for radiotherapy applications," Ph.D. dissertation, Carleton University, 2009.

[14] A. Shamim, E. Carleton University. Dissertation. Engineering, and Computer, "Wireless system-on-chip and system-on-package design for biomedical applications," Ph.D. dissertation, Carleton University, 2009.

[15] A. Shamim, M. Arsalan, L. Roy, M. Shams, and G. Tarr, "Wireless dosimeter: System-on-chip versus system-in-package for biomedical and space applications," IEEE Transactions on Circuits and Systems II: Express Briefs, vol. 55, no. 7, pp. 643-647, July 2008.

[16] M. Arsalan, A. Shamim, M. Shams, N. G., and L. Roy, "Ultra low power cmos-based sensor for on-body radiation dose measurements," IEEE Journal on Emerging and Selected Topics in Circuits and Systems, vol. 2, no. 1, pp. 34-41, March 2012.

[17] S. Toth, E. Carleton University. Dissertation. Engineering, and Computer, "An effcient rf rectifier for energy harvesting systems with applications to wireless dosimetry," Master's thesis, Carleton University, 2014.

[18] K. Finkenzeller, RFID Handbook: Fundamentals and Applications in Contactless Smart Cards, Radio Frequency Identification and near-Field Communication, Third Edition, 3rd ed. Wiley Online Book, 2010.

[19] B. Wang, M. K. Law, A. Bermak, and H. C. Luong, "A passive rfid tag embedded temperature sensor with improved process spreads immunity for a $-30^{\circ} \mathrm{C}$ 
to $60^{\circ} \mathrm{c}$ sensing range," IEEE Transactions on Circuits and Systems I: Regular Papers, vol. 61, no. 2, pp. 337-346, Feb 2014.

[20] S. Park, C. Min, and S. Cho, "A 95nw ring oscillator-based temperature sensor for rfid tags in 0.13 um cmos," in 2009 IEEE International Symposium on Circuits and Systems, May 2009, pp. 1153-1156.

[21] G. C. Martins and F. R. de Sousa, "An rf-powered temperature sensor designed for biomedical applications," in 2013 26th Symposium on Integrated Circuits and Systems Design (SBCCI), Sept 2013, pp. 1-6.

[22] D. M. Dobkin, The RF in RFID: UHF RFID in Practice, 2nd ed. Newnes, 2012 .

[23] H. Dagan, A. Teman, A. Fish, E. Pikhay, V. Dayan, and Y. Roizin, "A low-cost low-power non-volatile memory for rfid applications," in 2012 IEEE International Symposium on Circuits and Systems, May 2012, pp. 1827-1830.

[24] P. Feng, Y. Li, and N. Wu, "An ultra low power non-volatile memory in standard cmos process for passive rfid tags," in 2009 IEEE Custom Integrated Circuits Conference, Sept 2009, pp. 713-716.

[25] K. F. Strauss and T. Daud, "Overview of radiation tolerant unlimited write cycle non-volatile memory," in 2000 IEEE Aerospace Conference. Proceedings (Cat. No.00TH8484), vol. 5, 2000, pp. 399-408 vol.5.

[26] I. Microelectronics, Design kit and technology training CMOS8RF (CMRF8SF) V1700. unpublished, Oct 2010.

[27] M. N. Martin, D. R. Roth, A. Garrison-Darrin, P. J. McNulty, and A. G. Andreou, "Fgmos dosimetry: design and implementation," IEEE Transactions on Nuclear Science, vol. 48, no. 6, pp. 2050-2055, Dec 2001.

[28] E. Isern, M. Roca, E. Garcia-Moreno, J. C. Font, J. Cesari, and A. Pineda, "Characterization of a floating-gate radiation sensor for x-ray dose detection," in 2013 14th European Conference on Radiation and Its Effects on Components and Systems (RADECS), Sept 2013, pp. 1-4.

[29] E. Garcia-Moreno, E. Isern, M. Roca, R. Picos, J. Font, J. Cesari, and A. Pineda, "Floating gate cmos dosimeter with frequency output," IEEE Transactions on Nuclear Science, vol. 59, no. 2, pp. 373-378, April 2012.

[30] E. Garcia-Moreno and E. Isern, "Temperature compensated floating gate mos 
radiation sensor with current output," IEEE Transactions on Nuclear Science, vol. 60, no. 5, pp. 4026-4030, Oct 2013.

[31] B. Yadegari, E. Carleton University. Dissertation. Engineering, and Computer, Low Power Gamma-ray FG-MOSFET Dosimeter in 0.13 um CMOS Technology, 2012. [Online]. Available: https://books.google.ca/books?id= -KCBMwEACAAJ

[32] S. Danzeca, J. Cesari, M. Brugger, L. Dusseau, A. Masi, A. Pineda, and G. Spiezia, "Characterization and modeling of a floating gate dosimeter with gamma and protons at various energies," IEEE Transactions on Nuclear Science, vol. 61, no. 6, pp. 3451-3457, Dec 2014.

[33] Y. Wang, Z. Xiang, H. Hu, and F. Cao, "Feasibility study of semifloating gate transistor gamma-ray dosimeter," IEEE Electron Device Letters, vol. 36, no. 2, pp. 99-101, Feb 2015.

[34] P. Evgeny, R. Yakov, and N. Yael, "Ultra-low power consuming direct radiation sensors based on floating gate structures," Journal of Low Power Electronics and Applications, vol. 7, no. 3, 2017. [Online]. Available: http://www.mdpi.com/2079-9268/7/3/20

[35] A. Faigon, I. M. Vazquez, S. Carbonetto, M. G. Inza, and G, "Floating gate sensor for in-vivo dosimetry in radiation therapies. design and first characterization." Journal of Physics: Conference Series, vol. 792, no. 1, p. 012057, 2017. [Online]. Available: http://stacks.iop.org/1742-6596/792/i=1/ $\mathrm{a}=012057$

[36] M. S. Nadjar, E. Carleton University. Dissertation. Engineering, and Computer, "Robust subthreshold cmos voltage-controlled frequency synthesizer for wireless dosimeter application," Master's thesis, Carleton University, 2015. [Online]. Available: http://www.collectionscanada.gc.ca/obj/thesescanada/ vol2/OOCC/TC-OOCC-18913.pdf

[37] A. P. C. Alice Wang, Benton Highsmith Calhoun, Sub-threshold Design for Ultra Low-Power Systems, 1st ed., ser. Integrated Circuits and Systems. Springer US, 2006.

[38] E. Mondot and J. P. David, "Experimental procedure influence on total dose 
cmos inverters hardness," in RADECS 93. Second European Conference on Radiation and its Effects on Components and Systems (Cat. No.93TH0616-3), Sept 1993, pp. 306-312.

[39] N. A. Estep, "High power microwave (hpm) and ionizing radiation effects on cmos devices," 2010.

[40] E. Pikhay, Y. Nemirovsky, Y. Roizin, V. Dayan, K. Lavrenkov, Y. Leibovich, and D. Epstein, "Radiation sensor based on a floating gate device," in 2012 IEEE 27th Convention of Electrical and Electronics Engineers in Israel, Nov 2012, pp. 1-4.

[41] E. Pikhay, Y. Roizin, U. Gatti, and C. Calligaro, "Re-usable 180nm cmos dosimeter based on a floating gate device," in 2016 IEEE International Conference on Electronics, Circuits and Systems (ICECS), Dec 2016, pp. 125-128.

[42] E. Pikhay, Y. Roizin, and Y. Nemirovsky, "Ultra-low power consuming direct radiation sensors based on floating gate structures," Journal of Low Power Electronics and Applications, vol. 7, no. 3, p. 20, Jul 2017. [Online]. Available: http://dx.doi.org/10.3390/jlpea7030020

[43] E. Pievanelli, R. Stefanelli, and D. Trinchero, "Wireless technology for occupational dosimetry implementing medium range sensor networks," in 2013 International Symposium on Electromagnetic Theory, May 2013, pp. 1144-1147.

[44] N. S. Mohamed, N. G. Wright, and A. B. Horsfall, "Self-powered x-ray sensors for extreme environments," in 2017 IEEE SENSORS, Oct 2017, pp. 1-3.

[45] E. Ebrard, B. Allard, P. Candelier, and P. Waltz, "Review of fuse and antifuse solutions for advanced standard cmos technologies," Microelectronics Journal, vol. 40, no. 12, pp. 1755 - 1765, 2009. [Online]. Available: http://www.sciencedirect.com/science/article/pii/S0026269209001748

[46] R. F. Rizzolo, T. G. Foote, J. M. Crafts, D. A. Grosch, T. O. Leung, D. J. Lund, B. L. Mechtly, B. J. Robbins, T. J. Slegel, M. J. Tremblay, and G. A. Wiedemeier, "Ibm system z9 efuse applications and methodology," IBM Journal of Research and Development, vol. 51, no. 1.2, pp. 65-75, Jan 2007.

[47] N. Robson, J. Safran, C. Kothandaraman, A. Cestero, X. Chen, R. Rajeevakumar, A. Leslie, D. Moy, T. Kirihata, and S. Iyer, "Electrically programmable fuse (efuse): From memory redundancy to autonomic chips," in 2007 IEEE Custom Integrated Circuits Conference, Sept 2007, pp. 799-804. 
[48] Y.-k. Kim, J.-h. Jang, G.-s. Yoon, D.-h. Lee, M.-y. Ha, P.-b. Ha, and Y.-h. Kim, "Design of 32-bit differential paired efuse otp memory in a form of two-dimensional array," Journal of Central South University, vol. 19, no. 12, pp. 3484-3491, Dec 2012. [Online]. Available: https: //doi.org/10.1007/s11771-012-1433-3

[49] D. Anand, B. Cowan, O. Farnsworth, P. Jakobsen, S. Oakland, M. R. Ouellette, and D. L. Wheater, "An on-chip self-repair calculation and fusing methodology," IEEE Design Test of Computers, vol. 20, no. 5, pp. 67-75, Sept 2003.

[50] J.-H. Jang, L.-y. Jin, H.-G. Jeon, K.-I. Kim, P.-B. Ha, and Y.-H. Kim, "Design of an 8 bit differential paired efuse otp memory ip reducing sensing resistance," Journal of Central South University, vol. 19, no. 1, pp. 168-173, Jan 2012. [Online]. Available: https://doi.org/10.1007/s11771-012-0987-4

[51] Y.-B. Park, I.-H. Choi, D.-H. Lee, L. Jin, J. H. Jang, P. B. Ha, and Y. H. Kim, "Design of an efuse otp memory of 8 bits based on a 0.35 um bcd process," in International Conference on Mobile IT Convergence, Sept 2011, pp. 137-139.

[52] K. Y. Na and Y. S. Kim, "Novel single polysilicon EEPROM cell with dual work function floating gate," IEEE Electron Device Letters, vol. 28, no. 2, pp. 151-153, 2007.

[53] J. Raszka, M. Advani, V. Tiwari, L. Varisco, N. Der Hacobian, A. Mittal, M. Han, A. Shirdel, and A. Shubat, "Embedded flash memory for security applications in a 0.13 um cmos logic process," vol. 47, pp. 46 - 512 Vol.1, 03 2004.

[54] F. Bedeschi, E. Bonizzoni, G. Casagrande, R. Gastaldi, C. Resta, G. Torelli, and D. ZelLa, "Set and reset pulse characterization in bjt-selected phase-change memories," in 2005 IEEE International Symposium on Circuits and Systems, May 2005, pp. 1270-1273 Vol. 2.

[55] F. Bedeschi, C. Resta, O. Khouri, E. Buda, L. Costa, M. Ferraro, F. Pellizzer, F. Ottogalli, A. Pirovano, M. Tosi, R. Bez, R. Gastaldi, and G. Casagrande, "An $8 \mathrm{mb}$ demonstrator for high-density $1.8 \mathrm{v}$ phase-change memories," in 2004 Symposium on VLSI Circuits. Digest of Technical Papers (IEEE Cat. No.04CH37525), June 2004, pp. 442-445.

[56] F. Bedeschi, R. Fackenthal, C. Resta, E. M. Donze, M. Jagasivamani, E. Buda, F. Pellizzer, D. Chow, A. Cabrini, G. M. A. Calvi, R. Faravelli, A. Fantini, 
G. Torelli, D. Mills, R. Gastaldi, and G. Casagrande, "A multi-level-cell bipolarselected phase-change memory," in 2008 IEEE International Solid-State Circuits Conference - Digest of Technical Papers, Feb 2008, pp. 428-625.

[57] R. van Schaijk, M. van Duuren, P. Goarin, W. Y. Mei, and K. van der Jeugd, "Reliability of embedded sonos memories," in Proceedings of the 30th European Solid-State Circuits Conference (IEEE Cat. No.04EX850), Sept 2004, pp. 277280.

[58] S. S. Chung, Y. H. Tseng, C. S. Lai, Y. Y. Hsu, E. Ho, T. Chen, L. C. Peng, and C. H. Chu, "Novel ultra-low voltage and high-speed programming/erasing schemes for sonos flash memory with excellent data retention," in 2007 IEEE International Electron Devices Meeting, Dec 2007, pp. 457-460.

[59] J. F. Yang, L. F. Liu, B. Sun, H. Tang, N. Xu, Y. Wang, X. Y. Liu, R. Q. Han, J. F. Kang, and T. P. Ma, "Understanding the resistance switching mechanisms of binary metal oxides with the percolation model," in 2008 9th International Conference on Solid-State and Integrated-Circuit Technology, Oct 2008, pp. 940942.

[60] N. D. Phan, I. J. Chang, and J. W. Lee, "A 2-Kb one-time programmable memory for UHF passive RFID tag IC in a standard $0.18 \mu \mathrm{m}$ CMOS process," IEEE Transactions on Circuits and Systems I: Regular Papers, vol. 60, no. 7, pp. 1810-1822, 2013.

[61] M. C. Lee, R. Barsatan, and M. Chan, "Otp memory for low cost passive rfid tags," IEEE Conference on Electron Devices and Solid-State Circuits 2007, EDSSC 2007, pp. 633-636, 2007.

[62] H. Satake and A. Toriumi, "Sio 2 dielectric breakdown mechanism studied by the post-breakdown resistance statistics," Semiconductor Science and Technology, vol. 15, no. 5, p. 471, 2000. [Online]. Available: http://stacks.iop.org/0268-1242/15/i=5/a=306

[63] R. Degraeve, G. Groeseneken, R. Bellens, M. Depas, and H. Maes, "Consistent model for the thickness dependence of intrinsic breakdown in ultra-thin oxides," pp. 863 - 866, 011996.

[64] C. de Graaf, P. H. Woerlee, C. M. Hart, H. Lifka, P. W. H. de Vreede, P. J. M. Janssen, F. J. Sluijs, and G. M. Paulzen, "A novel high-density low-cost diode 
programmable read only memory," in International Electron Devices Meeting. Technical Digest, Dec 1996, pp. 189-192.

[65] J. Peng, G. Rosendale, M. Fliesler, D. Fong, J. Wang, C. Ng, Z. Liu, and H. Luan, "A novel embedded otp nvm using standard foundry cmos logic technology," in 2006 21st IEEE Non-Volatile Semiconductor Memory Workshop, Feb 2006, pp. 24-26.

[66] P. Candelier, N. Villani, J. P. Schoellkopf, and P. Mortini, "One time programmable drift antifuse cell reliability," in 2000 IEEE International Reliability Physics Symposium Proceedings. 38th Annual (Cat. No.00CH37059), 2000, pp. 169-173.

[67] J. Kim and K. Lee, "3-transistor antifuse otp rom array using standard cmos process," in 2003 Symposium on VLSI Circuits. Digest of Technical Papers (IEEE Cat. No.03CH37408), June 2003, pp. 239-242.

[68] K. Jinbong and L. Kwyro, "Three-transistor one-time programmable (otp) rom cell array using standard cmos gate oxide antifuse," vol. 24, pp. 589 - 591, 10 2003.

[69] H. K. Cha, I. Yun, J. Kim, B. C. So, K. Chun, I. Nam, and K. Lee, "A 32$\mathrm{kb}$ standard cmos antifuse one-time programmable rom embedded in a 16-bit microcontroller," IEEE Journal of Solid-State Circuits, vol. 41, no. 9, pp. 21152124, Sept 2006.

[70] J. Yin, J. Yi, M. K. Law, Y. Ling, M. C. Lee, K. P. Ng, B. Gao, H. C. Luong, A. Bermak, M. Chan, W. H. Ki, C. Y. Tsui, and M. Yuen, "A system-onchip epc gen-2 passive uhf rfid tag with embedded temperature sensor," IEEE Journal of Solid-State Circuits, vol. 45, no. 11, pp. 2404-2420, Nov 2010.

[71] N. Chen. (2016) Which nvm works best in mobile payments, iot and other applications. [Online]. Available: http://semiengineering.com/ the-benefits-of-antifuse-otp/

[72] C. Kothandaraman, S. K. Iyer, and S. S. Iyer, "Electrically programmable fuse (efuse) using electromigration in silicides," IEEE Electron Device Letters, vol. 23, no. 9, pp. 523-525, Sept 2002.

[73] T. S. Doorn and M. Altheimer, "Ultra-fast programming of silicided polysilicon 
fuses based on new insights in the programming physics," in IEEE InternationalElectron Devices Meeting, 2005. IEDM Technical Digest., Dec 2005, pp. 667-670.

[74] C. Tian, B. Park, C. Kothandaraman, J. Safran, D. Kim, N. Robson, and S. Iyer, "Reliability qualification of cosi2 electrical fuse for 90nm technology," in 2006 IEEE International Reliability Physics Symposium Proceedings, March 2006, pp. 392-397.

[75] J. Safran, A. Leslie, G. Fredeman, C. Kothandaraman, A. Cestero, X. Chen, R. Rajeevakumar, D. k. Kim, Y. Z. Li, D. Moy, N. Robson, T. Kirihata, and S. Iyer, "A compact efuse programmable array memory for soi cmos," in 2007 IEEE Symposium on VLSI Circuits, June 2007, pp. 72-73.

[76] N. Robson, J. Safran, C. Kothandaraman, A. Cestero, X. Chen, R. Rajeevakumar, A. Leslie, D. Moy, T. Kirihata, and S. Iyer, "Electrically programmable fuse (efuse): From memory redundancy to autonomic chips," in 2007 IEEE Custom Integrated Circuits Conference, Sept 2007, pp. 799-804.

[77] J.-H. Kim, D.-H. Kim, L. Jin, P.-B. Ha, and Y.-H. Kim, "Design of 1-kb efuse otp memory ip with reliability considered," in 2011 JOURNAL OF SEMICONDUCTOR TECHNOLOGY AND SCIENCE, vol. 11, no. 2, June 2011, pp. 88-94.

[78] G. Uhlmann, T. Aipperspach, T. Kirihata, C. Kothandaraman, Y. Z. Li, C. Paone, B. Reed, N. Robson, J. Safran, D. Schmitt, and S. Iyer, "A commercial field-programmable dense efuse array memory with 99.999soi cmos," in 2008 IEEE International Solid-State Circuits Conference - Digest of Technical Papers, Feb 2008, pp. 406-407.

[79] Y. Wang, N. G. Tarr, K. Shortt, and I. Thomson, "A sensitive floating gate mosfet gamma ray dosimeter," in Proceedings of IEEE Sensors 2003 (IEEE Cat. No.03CH37498), vol. 2, Oct 2003, pp. 1271-1276 Vol.2.

[80] R. Ferraro, S. Danzeca, M. Brucoli, A. Masi, M. Brugger, and L. Dilillo, "Design of a radiation tolerant system for total ionizing dose monitoring using floating gate and radfet dosimeters," Journal of Instrumentation, vol. 12, no. 04, p. C04007, 2017. [Online]. Available: http://stacks.iop.org/1748-0221/12/i=04/a=C04007

[81] B. T. Ltd. (2011) Raycell mk2 x-ray blood irradiator, 2.01 or 
3.51 canisters. [Online]. Available: http://www.theratronics.ca/PDFs/ RCMK2_BTMBTS_8014RCMK2_4_v112011_web.pdf

[82] E. Gullikson, A. Mills, and J. M. Phillips, "Electron and positron work functions of cobalt silicide," Surface Science, vol. 195, no. 1, pp. L150 - L154, 1988. [Online]. Available: http://www.sciencedirect.com/science/article/pii/ 0039602888907741

[83] K. Saraswat. (2017) Polycides, salicides and metals gates. [Online]. Available: https://web.stanford.edu/class/ee311/NOTES/Silicides\%20\& \%20Metal\%20gate\%20Slides.pdf

[84] S. M. Sze, Physics of Semiconductor Devices, 2nd ed. John Wiley and Sons, 1981.

[85] T. Cheung, M. J. Butson, and P. K. N. Yu, "Effects of temperature variation on mosfet dosimetry," Physics in Medicine and Biology, vol. 49, no. 13, p. N191, 2004. [Online]. Available: http://stacks.iop.org/0031-9155/49/i=13/a=N02

[86] A. B. Rosenfeld, M. C. Carolan, G. I. Kaplan, B. J. Allen, and V. I. Khivrich, "Mosfet dosimeters: the role of encapsulation on dosimetric characteristics in mixed gamma-neutron and megavoltage x-ray fields," IEEE Transactions on Nuclear Science, vol. 42, no. 6, pp. 1870-1877, Dec 1995.

[87] A. B. Rosenfeld, G. I. Kaplan, T. Kron, B. J. Allen, A. Dilmanian, I. Orion, B. Ren, M. L. F. Lerch, and A. Holmes-Siedle, "Mosfet dosimetry of an x-ray microbeam," IEEE Transactions on Nuclear Science, vol. 46, no. 6, pp. 17741780, Dec 1999.

[88] M. S. Andjelković, G. S. Ristić, and A. B. Jakšić, "Using radfet for the real-time measurement of gamma radiation dose rate," Measurement Science and Technology, vol. 26, no. 2, p. 025004, 2015. [Online]. Available: http://stacks.iop.org/0957-0233/26/i=2/a=025004

[89] N. H. E. Weste and D. M. Harris, CMOS VLSI Design, A Circuit and Systems Perspective, 4th ed. Pearson, 2011.

[90] M. Alioto, "Ultra-low power vlsi circuit design demystified and explained: A tutorial," IEEE Transactions on Circuits and Systems I: Regular Papers, vol. 59, no. 1, pp. 3-29, Jan 2012.

[91] D. I. Luna. (2015) Photodiode blue $0.74 \mathrm{~mm}$ sq smd. [Online]. 
Available: https://www.digikey.ca/product-detail/en/advanced-photonix/ PDB-C152SM/PDB-C152SMCT-ND/480629

[92] M. K. Law and A. Bermak, "High-voltage generation with stacked photodiodes in standard cmos process," IEEE Electron Device Letters, vol. 31, no. 12, pp. 1425-1427, Dec 2010.

[93] W. Xiaojuan and L. Jing, "Power sources and electrical recharging strategies for implantable medical devices," Frontiers of Energy and Power Engineering in China, vol. 2, no. 1, pp. 1-13, Mar 2008. [Online]. Available: https://doi.org/10.1007/s11708-008-0016-3

[94] I. Cevik and S. U. Ay, "A low-power and low-voltage power management strategy for on-chip micro solar cells," Sensors, Hindawi Publishing Corporation, vol. 2015, pp. 1-9, Apr 2015. [Online]. Available: https: //doi.org/10.1155/2015/739871

[95] M. Prauzek, J. Konecny, M. Borova, K. Janosova, J. Hlavica, and P. Musilek, "Energy harvesting sources, storage devices and system topologies for environmental wireless sensor networks: A review," Sensors (Basel), vol. 18, no. 8, Aug 2018.

[96] J. S. Lee, R. I. Hornsey, and D. Renshaw, "Analysis of cmos photodiodes. i. quantum efficiency," IEEE Transactions on Electron Devices, vol. 50, no. 5, pp. 1233-1238, May 2003.

[97] J. S. L. et al, "Analysis of cmos photodiodes. ii. lateral photoresponse," IEEE Transactions on Electron Devices, vol. 50, no. 5, pp. 1239-1245, May 2003.

[98] C. Honsberg and S. Bowden. (2013) Photovoltaic education network. [Online]. Available: https://www.pveducation.org/pvcdrom/pn-junctions/ absorption-depth

[99] P. M. Laboratory. (2004) X-ray mass attenuation coefficients. [Online]. Available: https://physics.nist.gov/PhysRefData/XrayMassCoef/chap3.html

[100] M. Nikl, "Scintillation detectors for x-rays," Measurement Science and Technology, vol. 17, no. 4, p. R37, 2006. [Online]. Available: http: //stacks.iop.org/0957-0233/17/i=4/a=R01

[101] J. G. Rocha and S. Lanceros-Mendez, "Review on x-ray detectors based on 
scintillators and cmos technology," Recent Patents on Electrical Engineering, vol. 4, pp. 16-41, 92011.

[102] P. A. Rodnyi, P. Dorenbos, and C. W. E. van Eijk, "Energy loss in inorganic scintillators," physica status solidi (b), vol. 187, no. 1, pp. 15-29. [Online]. Available: https://onlinelibrary.wiley.com/doi/abs/10.1002/pssb.2221870102

[103] D. J. Robbins, "On predicting the maximum efficiency of phosphor systems excited by ionizing radiation," Journal of The Electrochemical Society, vol. 127, no. 12, pp. 2694-2702, 1980. [Online]. Available: http://jes.ecsdl.org/content/127/12/2694.abstract

[104] Sigma-Aldrich. (2010) Yttrium oxide, europium doped. [Online]. Available: https://www.sigmaaldrich.com/catalog/product/aldrich/756490?lang= en\&region $=\mathrm{CA}$

[105] J. R. Jayaramaiah, B. N. Lakshminarasappa, and B. M. Nagabhushanac, "Luminescence studies of europium doped yttrium oxide nano phosphor," Sensors and Actuators B: Chemical, vol. 173, no. 12, pp. 234-238, 102012. [Online]. Available: https://doi.org/10.1016/j.snb.2012.06.092

[106] J. R. Jayaramaiah, B. N. Lakshminarasappa, and K. R. Nagabhushana, "Luminescence performance of europium doped yttrium oxide thin films," Journal of Luminescence, pp. 1-26, 8 2014. [Online]. Available: http: //dx.doi.org/10.1016/j.jlumin.2014.08.003

[107] Sigma-Aldrich. (2010) Cerium magnesium aluminate, terbium doped. [Online]. Available: https://www.sigmaaldrich.com/catalog/product/aldrich/756504? lang $=$ en\&region $=\mathrm{CA}$

[108] —. (2010) Barium magnesium aluminate, europium doped. [Online]. Available: https://www.sigmaaldrich.com/catalog/product/aldrich/756512? lang $=$ en\&region $=\mathrm{CA}$

[109] K. Yokota, S.-X. Zhang, K. Kimura, and A. Sakamoto, "Eu2+activated barium magnesium aluminate phosphor for plasma displays - phase relation and mechanism of thermal degradation," Journal of Luminescence, vol. 92, pp. 223-227, 02 2001. [Online]. Available: https://doi.org/10.1016/S0022-2313(00)00246-5

[110] T. Kobayashi, K. Nogami, T. Shirotori, and Y. Fujimoto, "A current-controlled 
latch sense amplifier and a static power-saving input buffer for low-power architecture," IEEE Journal of Solid-State Circuits, vol. 28, no. 4, pp. 523-527, Apr 1993.

[111] B. Razavi, "The strongarm latch [a circuit for all seasons]," IEEE Solid-State Circuits Magazine, vol. 7, no. 2, pp. 12-17, Spring 2015. 